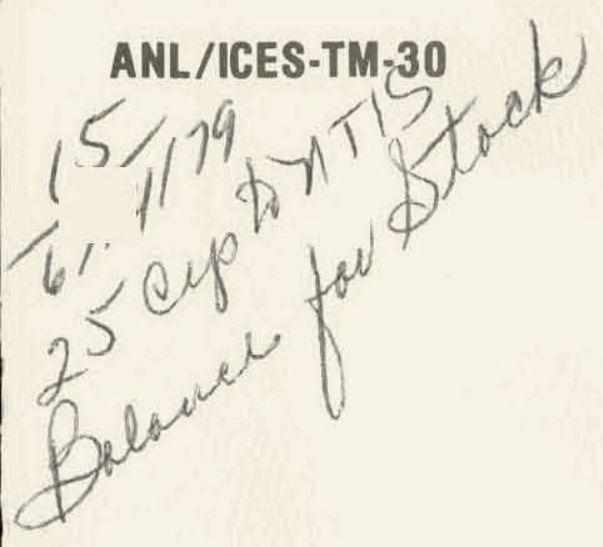

16.2716

\title{
HEAT PUMP CENTERED INTEGRATED COMMUNITY ENERGY SYSTEMS
}

\author{
System Development
}

University of Alabama Interim Report

\section{ARGONNE NATIONAL LABORATORY}

ENERGY AND ENVIRONMENTAL SYSTEMS

INTEGRATED

COMMUNITY DIVISION 


\section{DISCLAIMER}

This report was prepared as an account of work sponsored by an agency of the United States Government. Neither the United States Government nor any agency Thereof, nor any of their employees, makes any warranty, express or implied, or assumes any legal liability or responsibility for the accuracy, completeness, or usefulness of any information, apparatus, product, or process disclosed, or represents that its use would not infringe privately owned rights. Reference herein to any specific commercial product, process, or service by trade name, trademark, manufacturer, or otherwise does not necessarily constitute or imply its endorsement, recommendation, or favoring by the United States Government or any agency thereof. The views and opinions of authors expressed herein do not necessarily state or reflect those of the United States Government or any agency thereof. 


\section{DISCLAIMER}

Portions of this document may be illegible in electronic image products. Images are produced from the best available original document. 
The facilities of Argonne National Laboratory are owned by the United States Government. Under the terms of a contract (W-31-109-Eng-38) among the U. S. Department of Energy, Argonne Universities Association and The University of Chicago, the University employs the staff and operates the Laboratory in accordance with policies and programs formulated, approved and reviewed by the Association.

\section{MEMBERS OF ARGONNE UNIVERSITIES ASSOCIATION}

The University of Arizona Carnegie-Mellon University Case Western Reserve University The University of Chicago University of Cincinnati IIlinois Institute of Technology University of Illinois Indiana University The University of lowa lowa State University
The University of Kansas Kansas State University Loyola University of Chicago Marquette University The University of Michigan Michigan State University University of Minnesota University of Missouri Northwestern University University of Notre Dame
The Ohio State University Ohio University

The Pennsylvania State University Purdue University Saint Louis University Southern Illinois University The University of Texas at Austin Washington University Wayne State University The University of Wisconsin-Madison

\section{NOTICE}

This report was prepared as an account of work sponsored by the United States Government. Neither the United States nor the United States Department of Energy, nor any of their employees, nor any of their contractors, subcontractors, or their employees, makes any warranty, express or implied, or assumes any legal liability or responsibility for the accuracy, completeness or usefulness of any information, apparatus, product or process disclosed, or represents that its use would not infringe privately-owned rights. Mention of commercial products, their manufacturers, or their suppliers in this publication does not imply or connote approval or disapproval of the product by Argonne National Laboratory or the U. S. Department of Energy. 
ARGONNE NATIONAL LABORATORY

9700 South Cass Avenue

Argonne, I1linois . 60439

HEAT PUMP CENTERED

INTEGRATED COMMUNITY ENERGY SYSTEMS

System Development

University of Alabama Interim Report

\author{
By \\ Walter J. Schaetzle \\ and \\ C. Everett Brett
}

Bureau of Engineering Research and Natural Resources Center THE UNIVERSITY OF ALABAMA University, Alabama 35486

\section{Prepared for}

Energy and Environmental Systems Division Argonne National Laboratory under Argonne Contract No. 31-109-38-4550

Argonne Project Manager: James M. Calm, PE

March 1979

Work sponsored by

U.S. Department of Energy

Assistant Secretaryifor. Conservation and Solar Applications

Office of Buildings and Community Systems

Community Systems Branch 
I. System Description

A. Controlled Environment System

B. Thermal Energy Storage

C. Well System

D. Water Distribution System

E. Additions of Ground Water, Waste Heat, and Solar Energy $\quad 17$

F. The Public Service Utility

G. Minor Related Systems

H. Summary

II. Potential Applications

III. Expected Performance

IV. Expected Economics

V. Expected Environmental Impacts $\quad 64$

VI. Projected Growth 69

VII. Identified Variations $\quad . \quad 71$

VIII. Component Testing Requirements . 78

IX. Analysis Descriptions $\quad \therefore \quad \cdots 9$

A. Controlled Environmental System - Heat Pump Performance 79.

B. Aquifer Analysis 100

C. Welis 116

D. Water Distribution System 123

E. Heat Exchanger Fouling . . 131

F. Sular Energy Addition 136

G. Minor Related Systems ' 140

X. References $\quad \ldots \quad 146$

XI. Appendix I - Annual Heating and Cooling Performance Computer Program Summary

XII. Appendix II - Computer Program for Determining Pressure Loss in Piping System Due to Friction 


\section{List of Figures}

Page

1 - Schematic of the Technical Components for the Proposed Heat Pump Centered Integrated Community Energy System

2 - Standard Heat Pump System Module in Heat Pump Integrated Community Energy System

3 - Groups of Heat Pump System Modules in Heat Pump Integrated Community Energy System

4 - Standard Well Pair for 1 Module

5. 2 Aquifer System for 1 Module System

6 - Aquifer Thermal Energy Storage Capacity

7 - Schematic of Well Pumping System

8 - Module Total Distribution Pipe Pressure Loss

9 - Module Total Distribution Pipe Pressure Loss

10 - Heating to Cooling Ratio Availability as a Function of Heat Pump Performance

11 - Adequate Aquifers for Proposed System in United States

12 - First Law of Thermodynamics Analysis

13 - Second Law of Thermodynamics Analysis

14 - Modification of Basic Water Source Heat Pump System

15 - Heat Pump Heating Performance Comparison

16 - Heat Pump Heating Capacity Comparison

17 - 1 Pipe System with Pump Circulating Water Through Heat. Exchanger (No Insulation on the Pipes)

18 - 2 Pipe System (No. Insulation on the Pipes)

19 - 3 Pipe System -- Central Chilling and Heating (2 Pipes Insulated)

20 - 4 Pipe System -- Central Heating and Chilling (Al1 Pipes Insulated)

21 - Average Temperatures of Shallow Ground Water in Degrees Fahrenheit 
22 - Laboratory Coefficient of Permeability, K, gal./day ft. ${ }^{2}$ at a

Hydraulic Gradient of $1 \mathrm{ft./ft.} \mathrm{(Modified} \mathrm{after} \mathrm{Todd,} \mathrm{1959)}$

23 - Well Pressure Drop as a Function Spacing and Permeability

24 - Ideal Streamline for Injection Well and Discharge Well with Equal Flow Rates without Ground Flow

25 - Streamlines for Injection and Discharge Wells with Equal Flow and Ground Flow in the Same Direction

26 - Streamlines for Injection and Discharge Wells with Equal Flow and Ground Flow Perpendicular to the Flow

27 - Streamlines for Injection and Discharge Wells with Equal Flow and Ground Flow Opposing

28 - Flow Rate vs. Distance

29 - Outcrops of the Eutaw Formation, Tuscaloosa Group, and Pottsville Formation in Alabama

30 - Outcrops of the Eutaw Formation, Tuscaloosa Group, Pottsville Formation and their Chronostratigraphic Equivalents

31 - Maximum Operating Pressure of Normal-Impact (Type I).PVC at any Temperature

32 - Time Effect on Heat Exchanger Fouling

33 - Measured Efficiency of Solar Collector

34 - Optimum Collector Inclination vs. Latitudes

35 - Standard Solar Collector System with Unglazed Panels

36 - Sketch of Hard Rubber Molded Solar Panel Without Glazing

37 - Panel Configuration for Solar Bag Collector 
List of Tables

1 - Performance of Water Source Heat Pump A in Proposed System

2 - System Performance for Modified Water Source Heat Pump A with Pre-Water to Air Heat Exchanger - Alternative I

3 - System Performance for Modified Water Source Heat Pump A with Pre-Water to Air Heat Exchanger - Alternative II

4 - System Performance for Modified Water Source Heat Pump A with Pre-Water to Air Heat Exchanger - Alternative III

5 - Energy Requirements for Heating Well-Insulated Typical Home

6 - Energy Requirements for Cooling Well-Insulated Tỵpical Home

7 - Energy Comparison for Heating and Cooling Well Insulated. Typical Home Percentage Savings Utilizing Proposed Water Source Heat Pump A

8 - Energy Comparison for Heating Well Insulated Typical Home Percentage Savings Utilizing Proposed Water Source Heat Pump A 46

9 - Energy Comparison for Cooling Well Insulated Typical Home Percentage Savings Utilizing Proposed Water Source Heat Pump-A

10 - Energy Comparison for Heating and Cooling Well Insulated Typical Home Percentage Savings Utilizing Proposed Modified Water Source Heat Pump A with Pre-Water Heat Exchanger -Alternative I

11 - Energy Comparison for Heating We11 Insulated Typical Home Percentage Savings Utilizing Proposed Modified Water Source Heat Pump A with Pre-Water Heat Exchanger -- Alternative I

12 - Energy Comparison for Cooling Well Insulated Typical Home Percentage Savings Utilizing Proposed Modified Water Source Heat Pump A w1th Pre-Water Heat Exchanger -- Alternative I

13 - Energy Comparison for Heating and Cooling Well Insulated Typical Home Percentage Savings Utilizing Proposed Modified Water Source Heat Pump $A$ with Pre-Water Heat Exchanger -Alternative II

14 - Energy Comparison for Heating Well Insulated Typical Home Percentage Savings Utilizing Proposed Modified Water Source Heat Pump A with Pre-Water Heat Exchanger -- Alternative II

15 - Energy Comparison for Cooling Well Insulated Typical Home Percentage Savings Utilizing Proposed Modified Water Source Heat Pump A with Pre-Water Heat Exchanger -- Alternative II 
16 - Energy Comparison for Heating and Cooling Well Insulated Typical Home Percentage Savings Utilizing Proposed Modified Water Source Heat Pump A with Pre-Water Heat Exchanger -A.t.ternative TII

17 - Energy Comparison for Heating Well Insulated Typical Home Percentage Savings Utilizing Proposed Modified Water Source Heat Pump A with Pre-Water Heat Exchanger -- Alternative III

18 - Energy Comparison for Cooling Well Insulated Typical Home Percentage Savings Utillzing Proposed Modified Water Source Heat Pump A with Pre-Water Heat Exchanger -- Alternative III

19 - Economic Analysis for Annual Heating and Cooling for Well Insulated Typical Home ( $\$ 0.04$ Per $\mathrm{Kw}-\mathrm{hr}$ Electric Cost)

20 - Economic Analysis for Annual Heating and Cooling for Well Insulated Typical Home ( $\$ 0.08$ Per $\mathrm{Kw}-\mathrm{hr}$ Electric Cost)

21 - Economic Analysis for Annual Heating and Cooling for Well Insulated Typical Home ( $\$ 0.12$ Per $\mathrm{KW}_{\mathrm{w}} \mathrm{hr}$ Electric Cost)

22 - Annual Hours in Five Degree Fahrenhiet Temperature Increments for Twenty-Four Cities

23 - Performance of Air-Source Heat Pump A

24 - Performance of Air-Source Heat Pump B

25 - Performance of Air-Source Heat Pump C

26 - Annual Performance for Temperature Variation in Twenty-Four Cities

27 - Annual Performance for Temperature Variation in Twenty-Four Cities

28. - Annual Performance for Temperature Variation in Twenty-Four Cities

29 - Annual Performance for Temperature Variation in Twenty-Four Cities

30 - Annual Performance for Temperature Variation in Twenty-Four Cities

31 - Annual Performance for Temperature Variation in Twenty-Four Cities

32 - Performance Computer Run 
Page

34 - Performance Computer Run $\quad \therefore \quad \therefore \quad 94$

35 - Performance Computer Run . . . . . 95

36 - Performance Computer Run 96

37 - Performance Computer Run 97

38 - Performance Computer Run . 98

39 - Average Water-Well Drilling Costs in the United States, $1978 \quad 120$

40 - Performance Tables for Submersible Pumps $\quad 122$

41 - Normal Physical Properties of PVC Piping Materials 124

42 - Commercial Sizes (IPS) and Weights of Polyvinyl Chloride (PVC) Pipe

43 - Cost of Distribution Pipe, Installation, and Trenching . 130

44 - Average Cooling Tower Water Quality, Runs 6-20 133

45 - Chemical Analyses of Water Samples from the Tuscaloosa Aquifers. (Kg-Gordo Formation, Kck-Coker Formation) 134

46 - Representative Chemical Analyses of Water from Wells Tapping the Principle Aquifer of the Eutaw Formation in Alabama.

47 - Solar Energy Collections on 100-Square Foot Horizontal Collector 
As the once popular perception of fuel being cheap and unlimited fades, increased emphasis is being given to more efficient use of energy resources and to the substitution of non-scarce energy forms for depleting and less abundant sources. Recognition of the need to conserve energy resources has been spurred in large part by the oil embargo of 1973 and subsequent escalation in fuel prices, by energy shortages in the harsh winter of $1976 / 77$, by increasing electricity "brown-outs" in urban centers, and by the crippling effects of labor strikes and stoppages in energy supply industries. In recent years, development and acceptance of energy conserving systems which avoid dependence on scarce or interruptible fuels has increased markedly in the United States.

One comprehensive approach to energy conservation is the Integrated Community Energy Systems (ICES) concept, which is intended to increase the efficiency of the varied ways in which energy is provided to and utilized by a community. At the same time, the ICES approach seeks to reduce dependence on scarce fuels and other resources, to preserve environmental quality, to offer energy and energy consuming services in an economically attractive manner, and, perhaps most importantly, to meet the energy needs of communities without adversely affecting social and lifestyle objectives.

The ICES approach to meeting these goals is embodied in three levels of integration. First, by incorporating innovative technology to maximize the resource utilization efficiency, energy requirements can be reduced. Included in this method are cogeneration (or more correctly coproduction) and cascading uses of energy to minimize the thermodynamic mismatch of source energy qualities and actual energy needs. Also included is fuel substitution in centralized commity-scale systems which would be impractical in independent, individual building and separate service energy systems. Second, by integrating the energy supply and energy consuming systems with the functional design and layout of the community, load management advantages can be achieved and. distribution losses can be minimized. At the same time, resource needs can be reduced through appropriate land utilization and planned growth. And third, the community systems development is integrated with the financial and regulatory mechanisms common to communities to permit widespread implementation.

A specific ICES may consist of efther a partial or complete integration of these approaches, as deemed necessary and appropriate to strike the desired balance among a community's economic, social, environmental, and energy conservation goals. An ICES can be applied to a total community as well as to portions of a community and the services provided need not be the same for all areas served. While ICES include a broad spectrum of technologies to meet energy service requirements, the ICES concept neither arbitrarily defines the energy services to be provided nor the type, size, or function of the area to be served. As a result, an ICES is tailored for each application and the determination of the kind and number of energy services provided and the size of the service area for each such service is based on an optimum combination of energy efficiencies, indigenous resource and labor supplies, economics, and environmental conditions.

The communities which ICES are intended to serve consist of multiple facilities in the residential, commercial, industrial, agricultural, recreational, or Institutional sectors or in some combination of sectors. 
Furthermore, these communities may be in various stages of development, e.g., planned, designed, constructed, or redevelopment.

Heat Pump Centered Integrated Community Energy Systems are energy systems for communities which provide heating, cooling, and/or other energy services through the use of heat pumps. Since heat pumps primarily transfer energy from existing and otherwise probably unused sources, rather than convert it from electrical or chemical to thermal form, HP-ICES are viewed as having significant potential for energy conservation. Furthermore, since conventional building heating and cooling systems would be replaced by this community energy system, nonscarce resources could be used instead of depleting fuels which are in short supply. This is accomplished by powering the heat pumps with nonscarce energy forms not practical for use in the smaller conventional systems. Secondary benefits expected to enhance the value of such systems include reduction of adverse environmental effects over conventional systems, reliable production of services in view of increasingly frequent utility curtailments and interruptions, and provision of services at costs more favorable to consumers than conventional system costs (including acquisition, operation, and maintenance).

The report which follows is a result of the System Development Phase of the HP-ICES Project. The objective of this multiphase project is development and demonstration of HP-ICES concepts leading to one or more operational systems by the end of 1983: The seven phases include System Development, Demonstration Design, Design Completion, HP-ICES Construction, Operation and Data Acquisition, HP-ICES Evaluation, and Upgraded Continuation.

This project is sponsored by the Community Systems Branch, Office of Buildings and Community Systems, Assistant Secretary for Conservation and Solar Systems, U. S. Department of Energy (DOE). It is a part of the Community Systems Program and is managed by the Energy and Environmental Systems Division of Argonne National Laboratory.

Nine parallel concepts are under development in the initial, System Development, phase. The report which follows presents the interim findings in the development and analysis of a concept being developed by The University of Alabama wi.th technical support from Alabama Power Company and American Air Filter. 
The heat pump centered integrated community energy system unifies groups of controlled environments (homes, businesses, etc.) into energy modules. Each module has an annual thermal energy storage system and water distribution system; and, in the controlled environments, each module has a water-source heat pump system. The modules can function independently or as part of a larger system composed of groups of modules. The components of the system outside the home and commercial units are serviced by a public utility. Large commercial units can economically install and operate independent energy modules. Original planning sets module size arbitrarily at 20 homes or environment control spaces or a large commercial building. In actuality, module size will vary with location and heating and cooling loads.

Technically, the heat pump system uses water-source heat pumps pareled with thermal energy storage in underground aquifers. The aquifer is a natural underground reservoir such as sand and gravel or porous rock in which water can be stored and transmitted. A central water distribution system circulates water between the aquifer and the heat pumps. In the summer, the thermal energy rejected by the heat pumps is stored in aquifers for use during the winter season. During the winter, the stored thermal energy is retrieved by the heat pump system. The rejected cooler water from the heat pump during heating periods is used to form a temperature heat sink in the aquifer for use during the cooling cycle. To equate heating and cooling, supplementary thermal energy must be added or removed from the system on an annual basis. Heat addition is accomplished by utilizing waste heat or solar-energy addition. A limited amount of heat 
removal is accomplished by rejecting warm watex from the aquifer or by placing a cooling tower in the system. Balancing the requirements of various types of buildings can be a partial solution.

Heat pump performance capability is primarily a function of the temperature difference at which the system must operate. If the difference between the source temperature and the controlled environment is large, the heat pump performance is poor. The smaller the temperature difference, the less entropy generated. An example of a large differential would be keeping a home at $72^{\circ} \mathrm{F}$ with an air-source heat pump using outside ambient temperature of $-10^{\circ} \mathrm{F}$ for a thermal energy source. If $80^{\circ}$ water, the minimum goal of this project, is used as a thermal energy source, performance of the heat pump system is improved. As a result, energy input to the pump is reduced. The situation is reversed during the cooling season with water temperatures below air temperatures.

Thermal energy storage is accomplished by using an underground aquifer as a reservoir. Two (or more) wells, penetrating the aquifer and spaced a predetermined distance apart, furnish water to the integrated energy system. During the cooling season, rejected warm water is injected into one well in the aquifer and relatively cold water is pumped from the aquifer through the second well to the heat pumps. The well spacing as related to aquifer storage capacity is determined so that a period of 6 months, or preferably longer, is required for the temperature gradient to move from one well to the second well. During the heating cycle, the water flow direction with respect to the 2 wells is reversed, and relatively warm water is recovered and pumped to the heat pumps.

Economically, the system shows improvement on both energy usage and capital costs. The system saves two-thirds of the energy required for 
resistance heating; saves $30 \%$ of the energy required for most air-source heat pumps (primary function of climatic location); and saves one-third of the energy required for gas, coal, or oil heating, compared to energy input required at the power plant for heat pump usage. Energy costs are less than the cost of gas or oil in some parts of the country. Capital costs also show an advantage when compared to a standard airsource heat pump system or to a gas, oil, or coal heating system plus an air conditioner. In general, for an individual residence or small commercial system, an air-source heat pump capital cost is approximately $100 \%$ more than an equivalent water-source heat pump with the same ARI capacity. For large commercial applications, most chillers already are water-source units connected to cooling towers. Under severe winter weather conditions, however, a water-source heat pump in this system will provide over 4 times more heating capacity than an ARI equally rated air-source heat pump. The difference in capital cost between the heat pump systems is offset by the cost of wells and the water distribution system.

The high performance air-source heat pump utilizes an improved compressor, improved blower and external fan motors, and larger heat transfer areas. These changes will be incorporated into water-source heat pumps over the next 2 years. Performance will improve between $20 \%$ and" $30 \%$, making the proposed system $15 \%$ to $25 \%$ more efficient than the performance analysis contained in this report.

Two identified modifications may be used to add a water-to-air heat exchanger to the water-source heat pump. The first increases performance by approximately $10 \%$; the second increases performance, on an annual basis, by $50 \%$ in some areas of the country, and eliminates the peaks in summer power plant output. 


\section{SYSTEM DESCRIPTION}

The heat pump centered integrated community energy system is designed in groups of integrated energy modules. The controlled environments in each module are served by individual water-source heat pumps. The heat pump could be a small unit in an individual room, a central heat pump for a residence, or an integrated heating and cooling system for a major building. Each module is served by a set of wells, an aquifer or aquifers, a pumping system, and a water distribution system. Figure 1 is a schematic of the general technical concept of the system module. The modules are designed so they can be combined to make larger integrated systems and thereby improve reliability, minimize emergency maintenance, minimize pressure losses, and allow for ordered expansion. Schematics of this module system are shown in Figures 2 and 3 . Figure 2 shows wells and pumping stations located in the right-of-way adjacent to roads, eliminating formal land use for these systems. The well outposts are expected to be no more conspicuous than the service outposts for underground distribution or telephone service.

The integrated system is divided into the following major components:

A. The controlled environmental system. This includes the controlled environment (house, apartment, small commercial building) and the individual water-source heat pumps. The controlled environment can be an individual room, apartment, or home served by a small individual heat pump or heat pump system. It can also be a large element such as a complete commercial or apartment building using a central water-source heat pump to heat or cool the individually controlled environments. The heat pumps can be water-to-water or water-to-air and may use one or more. stage compressors 


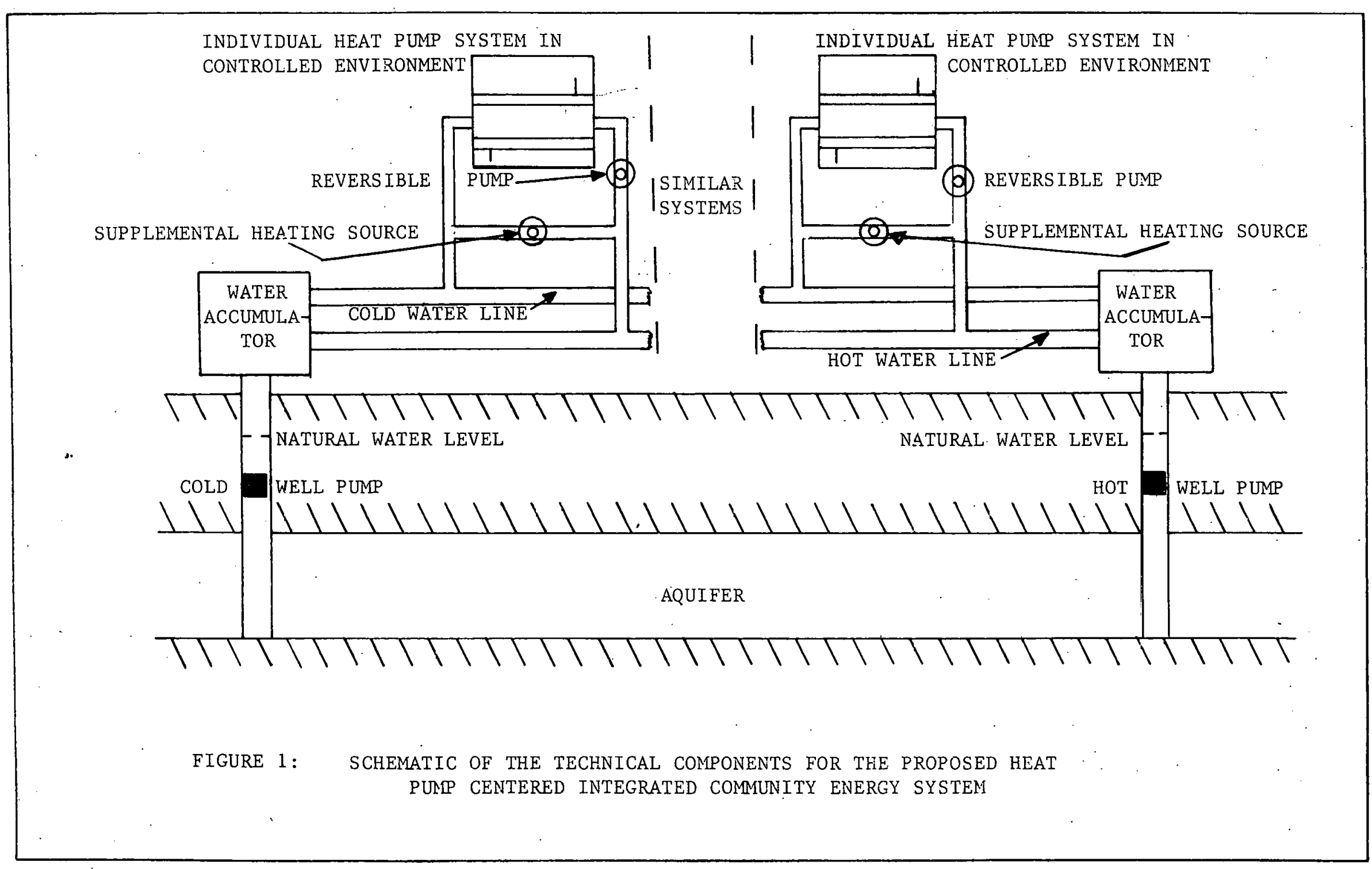




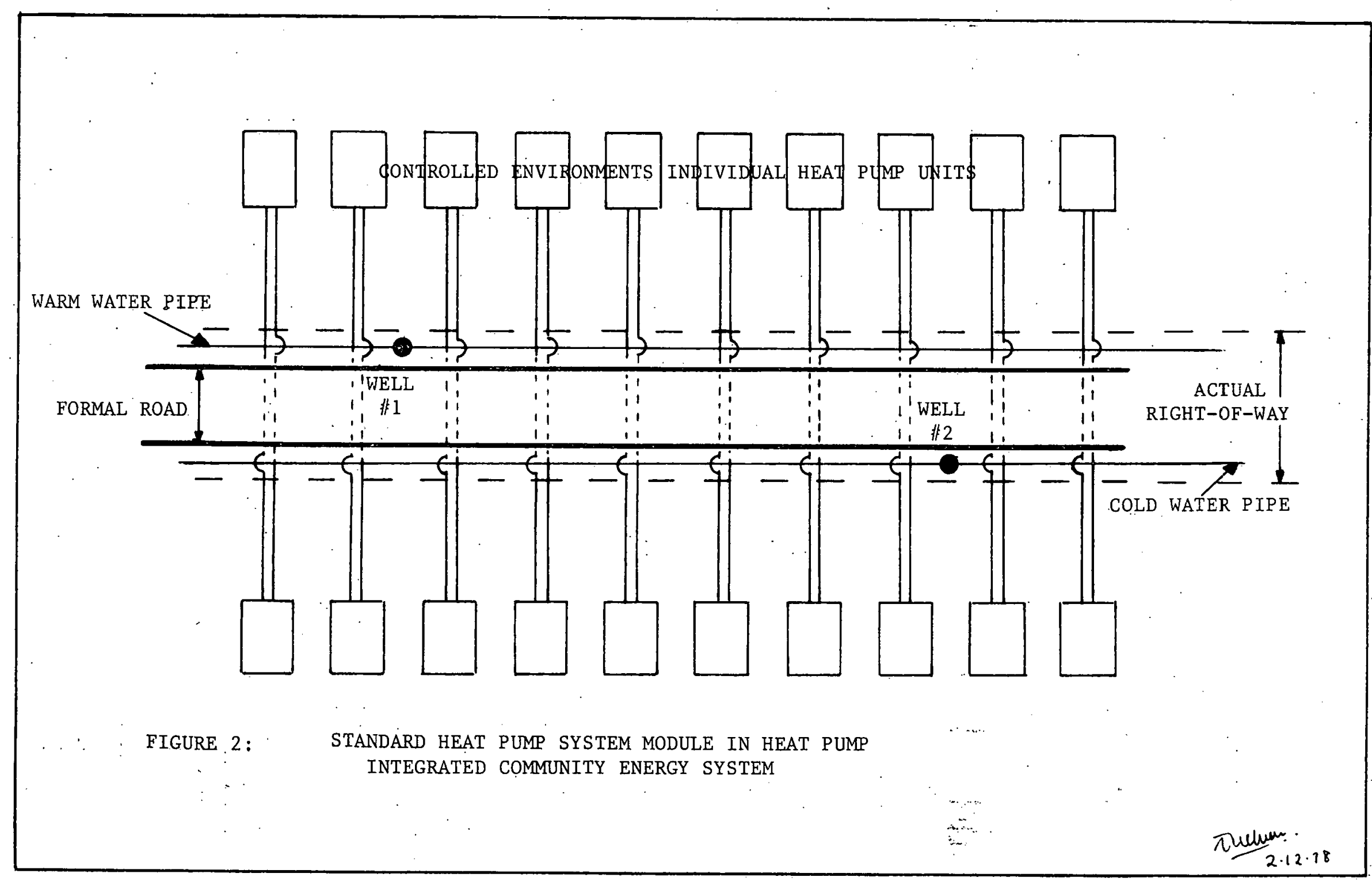




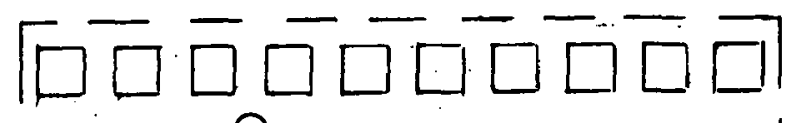 \\ $\odot$

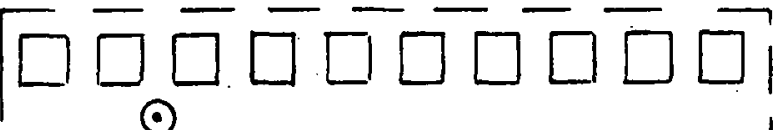 \\ $\odot$}

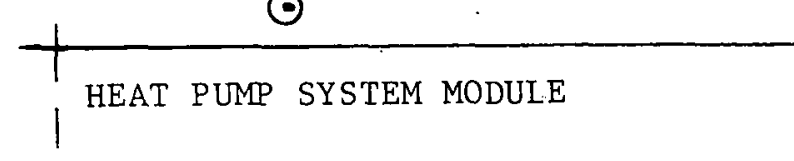

$10 \square$

$\square \square \square \square \square \square \square \square \square \square$

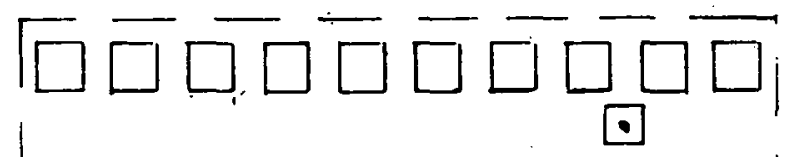

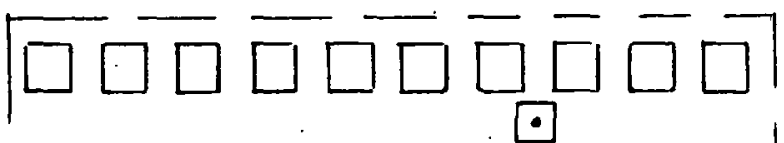

므므므맘ㅁㅁㅁㅁㅁㅁㅁㄴ

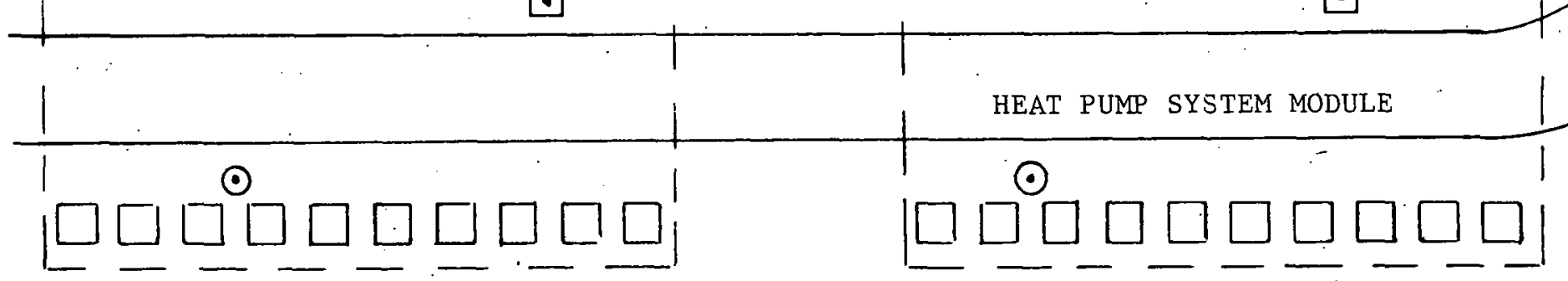

C WARM WELL

- COLD WELL 
of reciprocating, centrifugal, or screw configuration. Systems are expected to be off-the-shelf items.

B. The thermal energy storage module, or the aquifer. Aquifers are water-bearing rocks found near or at nominal depths below the soil zone of the earth's surface. Such rocks have physical properties of porosity and permeability, i.e. the presence of intergranular.voids, fractures, solution channels, or other interconnected openings capable of receiving, storing, and transmitting water from precipitation that infiltrates from the ground's surface. Commonly aquifers consist of rock of sedimentary origin, such as limestones, dolomites, siltstones, sandstones and gravels; although, in some instances, metamorphic and igneous rocks may contain water in sufficient quantities to serve as a source of fresh water. Approximately $60 \%$ of the surface of the continental United States is underlain by aquifers adequate for utilization in this system. Many urban areas are concentrated in valleys and near rivers where extensive aquifers exist. Thermal energy transfer is accomplished by transferring warm and cool water to and from the aquifers.

C. The wells which provide access to the aquifers. Wells consist of drilled holes, normally between 4 and 15 inches in diameter; or in some instances the holes may be hand-dug in which case diameters are somewhat larger. Each well will contain a submerged pump, controls, and a water tank with a capacity that may range between 20 and 200 gallons. D. The water distribution system. The water distribution system includes water lines and the controls between the aquifers and user 10cations. The lines provide the water flow to heat pumps and provide the water flow return to the aquifers. 
E. The auxiliary heat addition. To balance heating and cooling loads, additional energy is required in most parts of the country. This energy is applied by heat exchangers to source water, by waste energy sources, and/or by direct energy addition from solar collectors.

F. The public service utility. The public service utility will provide and service the wells, pumping systems, and water distribution system. In addition, some waste energy heat exchangers, solar systems, and heat pump exchangers could be provided and serviced by the utility.

G. Other minor system uses, such as providing water for fire protection. The benefit to the controlled environment is higher heating and cooling system performance. The system performance will be over 3 times that of resistance heating, will approach 2 times the performance of air-source heat pumps in some locations, and will increase by more than $30 \%$ the performance of oil, gas, or coal heating systems. These numbers are discussed in detail under Section III, Expected Performance. I.A. Controlled Environment System

Each controlled environment or section of the controlled environment has its own water source heat pump system. In the home, the general : heating and cooling system is no different from that of any other home (controlled environment). The heat pump unit is bought and installed by the home owner. The only difference is the water source which is required for the heat pump. A public utility will provide the water source.

The water is expected to be purchased for a monthly lump sum cost, similiar to electrical demand charge, based on the capacity of the heat pump system in the home. This charge rate, which is minor compared to heat pump energy usage cost, is expected to encourage energy conservation. 
For commercial buildings, water can also be provided for individual units or central units. The owner and consulting engineer must determine the best heating, ventilating, and air-conditioning system for the particular building. Any type water-source heat pump can be utilized with the system. The heat pump can be water-to-air or water-to-water with compression supplied by a reciprocating, screw, or centrifugal compressor. The use of cool intake water or of warm intake water supplied to the heat pump systems provides the increase in performance. The charge for water in large buildings could be economically measured as a function of energy or water flow rate. Flexible methods for charging are envisioned.

\section{I.B. Thermal Energy Storage}

Aquifers are used for energy storage and as energy sinks. The aquifer may. be comprised of sand, gravel, or porous rock which will store and allow the transmissivity of water. A suitable aquifer for this system will allow water recovery at a rate of one gal./min. per ft. of thickness. Optimum diameter of a well is considered to be 6 inches. As noted previously, aquifers of this quality are encountered in a large portion of the United States. Thermal energy is stored in the water-soil-rock combination. Two primary methods are used to store and retrieve the thermal energy. These are:

1. Two wells in the same aquifer where water is injected into one well and recovered in the second well (Figure 4). During the injection and recovery process, a water front and a temperature front (at lower velocity) move between the wells. In the cooling cycle, warmer water is injected in 1 well; , and cool water is withdrawn from the other and.: piped to the heat pump. For heating, the process is reversed and warm 


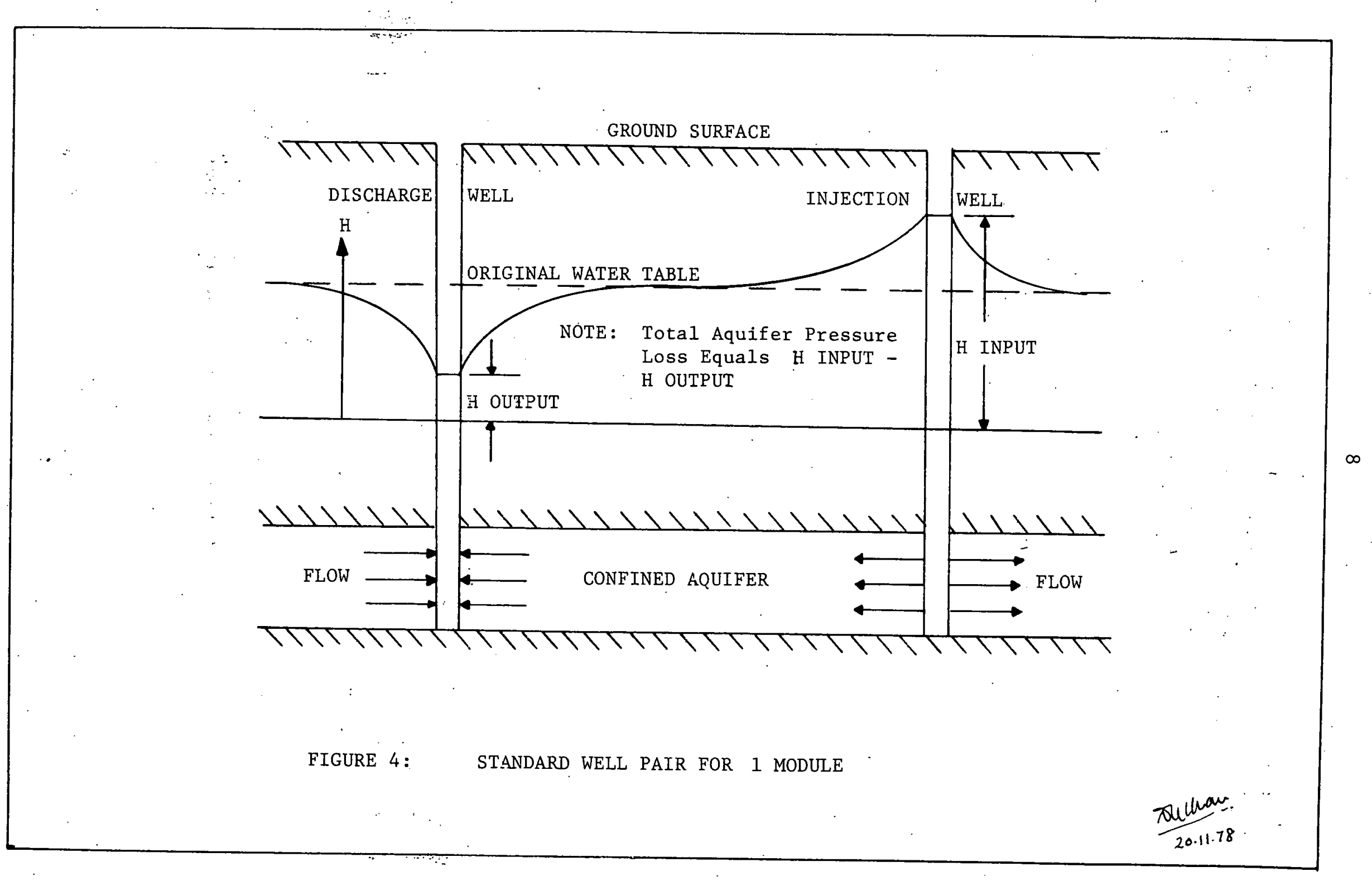


water is withdrawn and cold water injected. The optimum spacing between wells in the aquifer must be sufficiently large so the time for the thermal front (wave) to pass between the wells is greater than 6 months. Depending on withdrawal and injection rates, a spacing of a few hundred ft. between wells is considered sufficient. More than a single pair of wells in the same aquifer may be utilized. A more detailed analysis is given in Section IX.B.

2. Two aquifers are utilized with one aquifer storing relatively warm water and the second storing relatively cold water (Figure 5). In this case, cold water is recovered from the cold storage aquifer, pumped through the condenser of the heat pump for cooling, and injected as relatively warm water in the second aquifer. For heating, the process is reversed with warm water being recovered from the warm reservoir, pumped through the evaporator of the heat pump for heating, and injected as relatively cold water into the cold storage aquifer. An example would be isolated aquifers at depths of $80 \mathrm{ft}$. and $300 \mathrm{ft}$. where the aquifer at $80 \mathrm{ft}$. is originally lacking in sufficient water.

In both of the 2 examples of aquifer systems, water loss or gain on an anual basis would be negligible since recovered and injected water quantities are equal.

Thermal storage in aquifers is a function of porosity and type of rock. Most rock has a specific gravity of approximately 2.6 and a specific heat of about $0.2 \mathrm{Btu} /{ }^{\circ} \mathrm{F} /$ pound mass. Porosity can range from less than $5 \%$ to more than $30 \%$ with $10 \%$ to $20 \%$ as general averages for most aquifers. The thermal storage capacity per degree temperature change is just over $30 \mathrm{Btu} / \mathrm{ft}^{3}$, varying with porosity. At this value, 
GROUND SURFACE

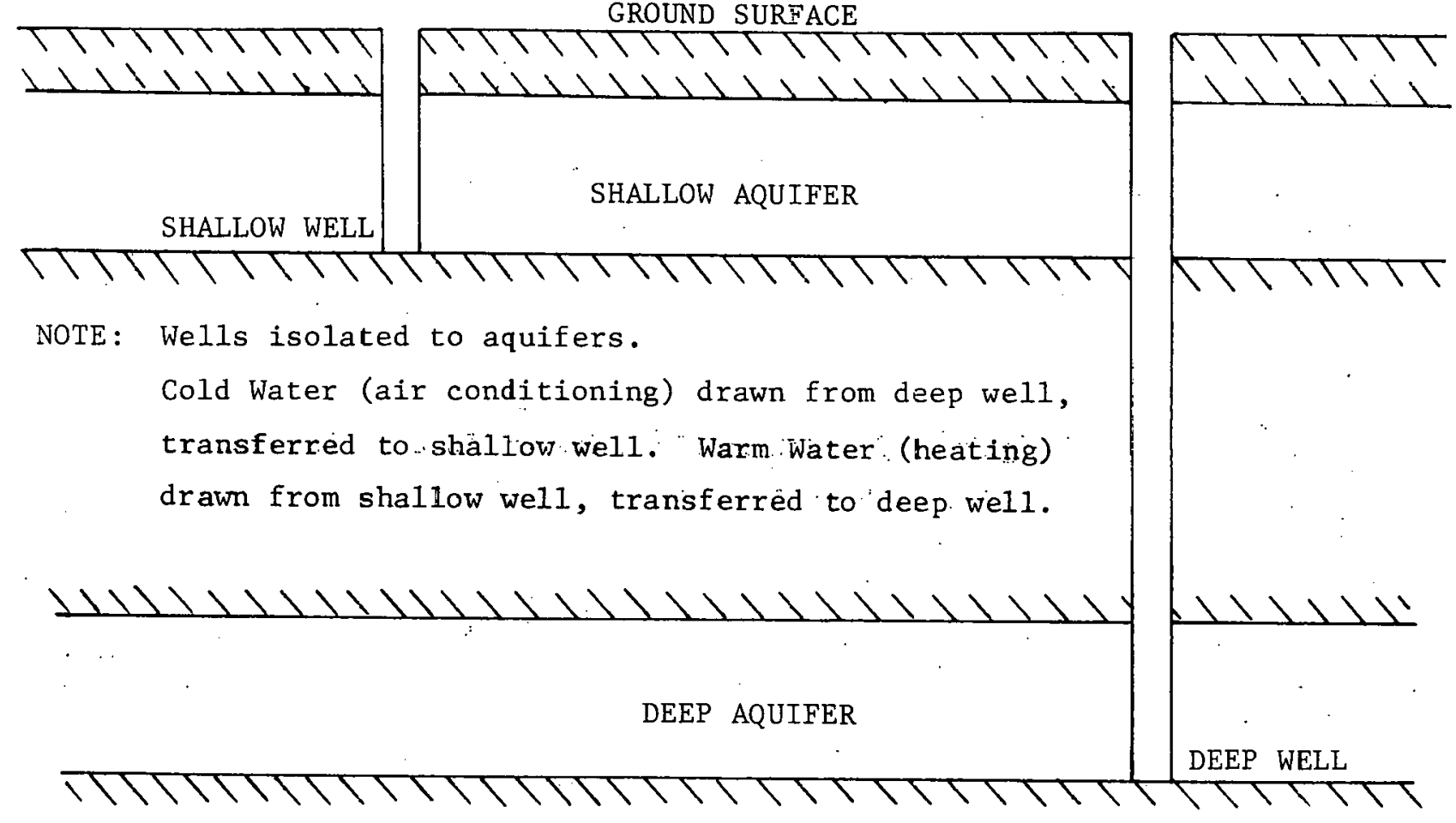

FIGURE 5: 2 AQUIFER SYSTEM FOR 1 MODULE SYSTEM 
a rock volume of $100 \mathrm{ft}$. by $200 \mathrm{ft}$. by $30 \mathrm{ft}$. has a storage capability of $2.0 \times 10^{8} \mathrm{Btu}$ with a $10^{\circ} \mathrm{F}$ temperature change. General values for the storage capacity of aquifers as a function of size, porosity, and temperature variations are given in Figure 6 :

Water velocities outside the immediate well vicinity: will be of the order of magnitude of one ft./day, and the temperature front velocity between wells is approximately one-half that of water velocity. Differences in the hydrostatic heat between wells will be less than $50 \mathrm{ft}$. More detailed values are given in the analysis section IX.C.

I.C. We11 System

A system of wells is required as a conduit for water between the aquifer and ground surface. Well diameters may range from 4 inches to 15 inches and depths from 20 to a few hundred ft. Well size and number of wells will be a function of water demand and aquifer characteristics, primarily those of permeability, porosity, and thickness. Water yield increases with diameter (logrithmic function). Well design for a particular aquifer will be established by a trade-off between well size and number of wells. Water per unit cost is the controlling factor. System reliability and legal and environmental requirements must be considered.

\section{I.D. Water Distribution System}

Water will be distributed from the wells to the controlled environments through an uninsulated 2-pipe pressurized water system. . This is shown schematically in Figure 1. Installed side-by-side with spacing between the 2, 1 pipe will carry relatively warm water and the other 


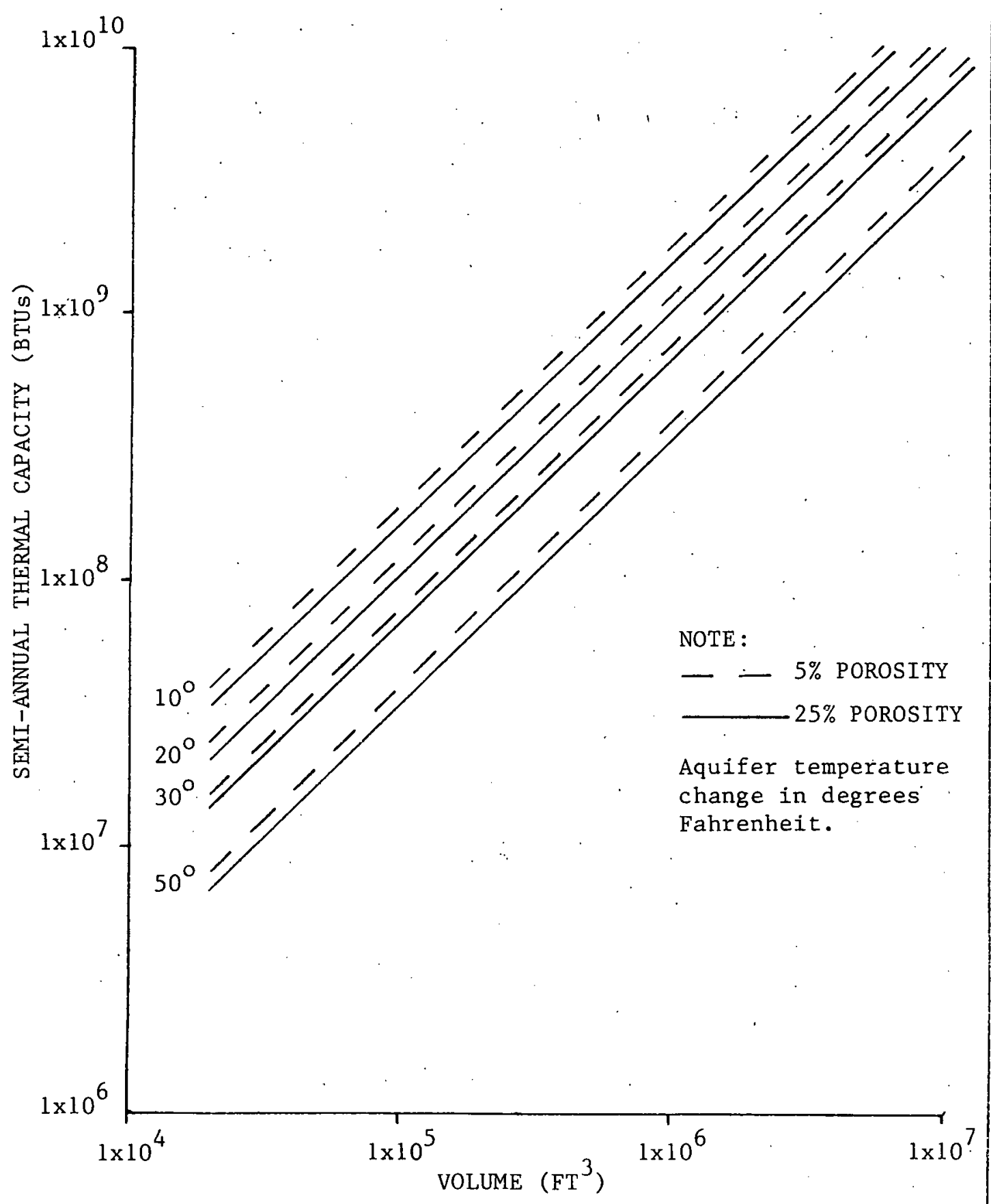

FIGURE 6: AQUIFER THERMAL ENERGY STORAGE CAPACITY 
relatively cold water. Each well will have a pumping system (Figure 7) consisting of a submerged pump, an accumulator system, and controls to provide a specific pressure in the pipelines. The accumulators at each well head: allow the pumps to operate at capacity for limited periods. The on-off sequences can be minimized in a group of modules by sequencing the pumps. A 2-pipe system from the primary pipe lines to the controlled environments (home, etc.) will include a separate small pump (on the order of $0.1 \mathrm{HP}$ ) to draw warm water or cold water as required from 1 primary line and reject the water to the other primary line. This allows the heat pumps to operate at peak performance at all times, with the capability of certain pumps cooling and other pumps heating simultaneously. At all times warm water is available for heating; and cold water is available for cooling, eliminating poor performance in a unit for heating during the cooling season or vice versa. The system also minimizes well pumping by thermal demand diversity. By using the module system, pressure losses in the main water lines are minimized as a result of short travel distances. Calculated hydrostatic heads for designed systems will be less than $30 \mathrm{ft}$. of water. Figures 8 and 9 give pressure losses for homes separated by $100 \mathrm{ft}$. and $200 \mathrm{ft}$., respectively, at various flow rates. Average maximum flow rate to a home is $6.0 \mathrm{GPM}$. Six-inch lines will be utilized as main water lines to provide adequate water for emergency purposes such as fire protection systems. It may also be noted the pressure loss in the main lines is well below 1 foot of water if 2 homes at opposite ends'of a module heat and cool simultaneously (flow rate of $10 \mathrm{GPM}$ ). Pressure loss in 


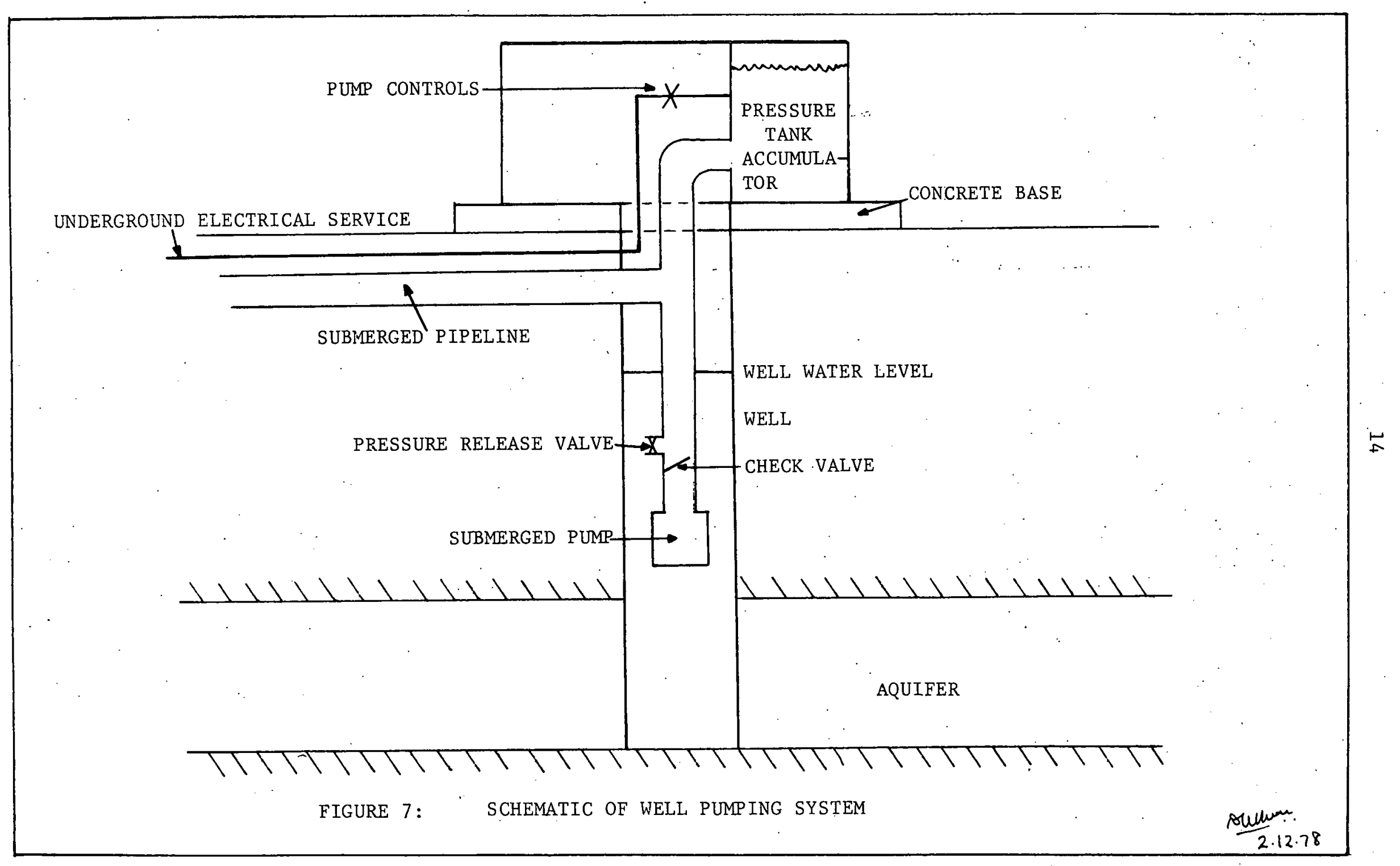


FIGURE 8: MODULE TOTAL DISTRIBUTION PIPE PRESSURE LOSS

20 HOME MODULE

100 FEET BETWEEN HOMES

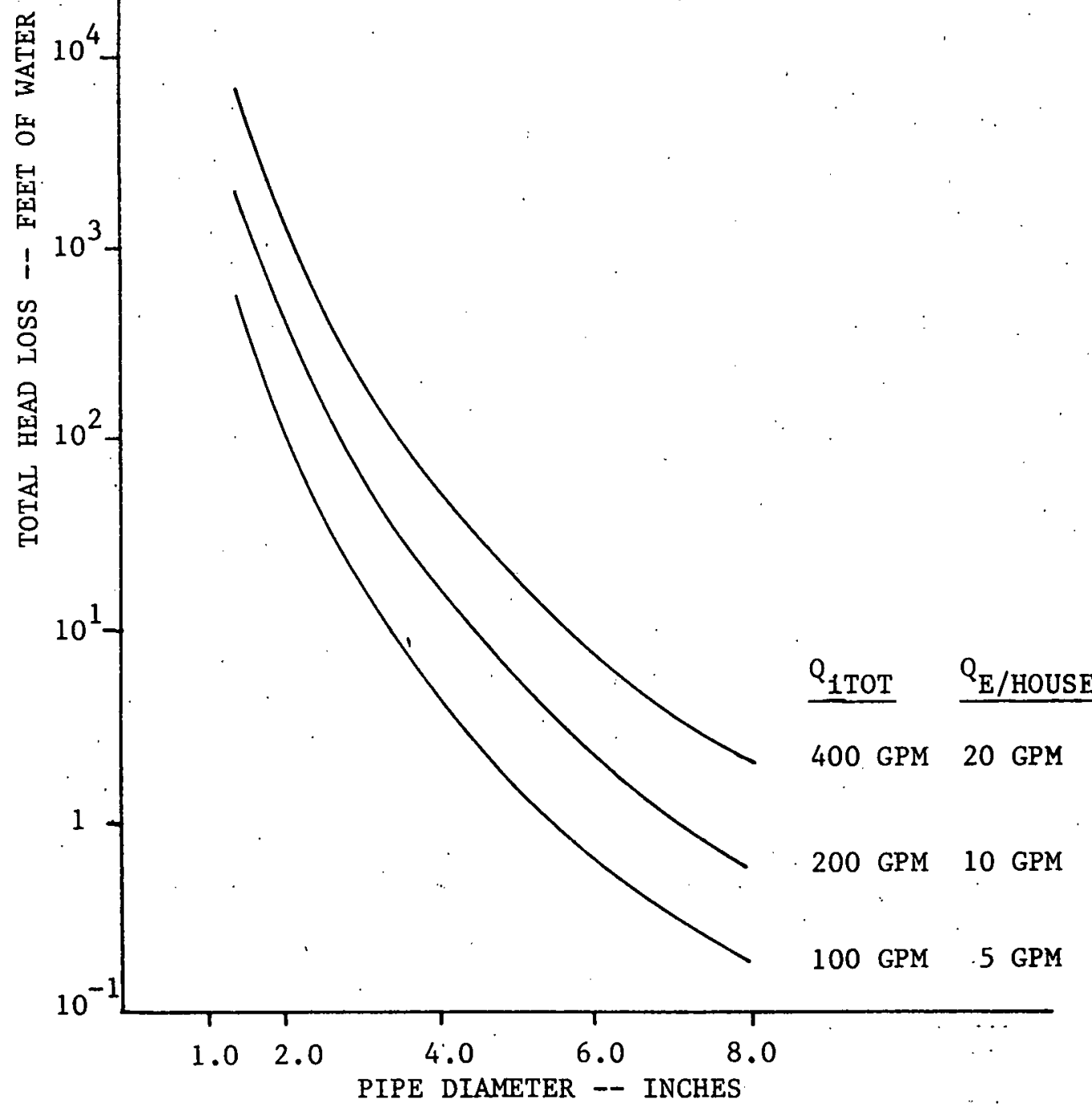




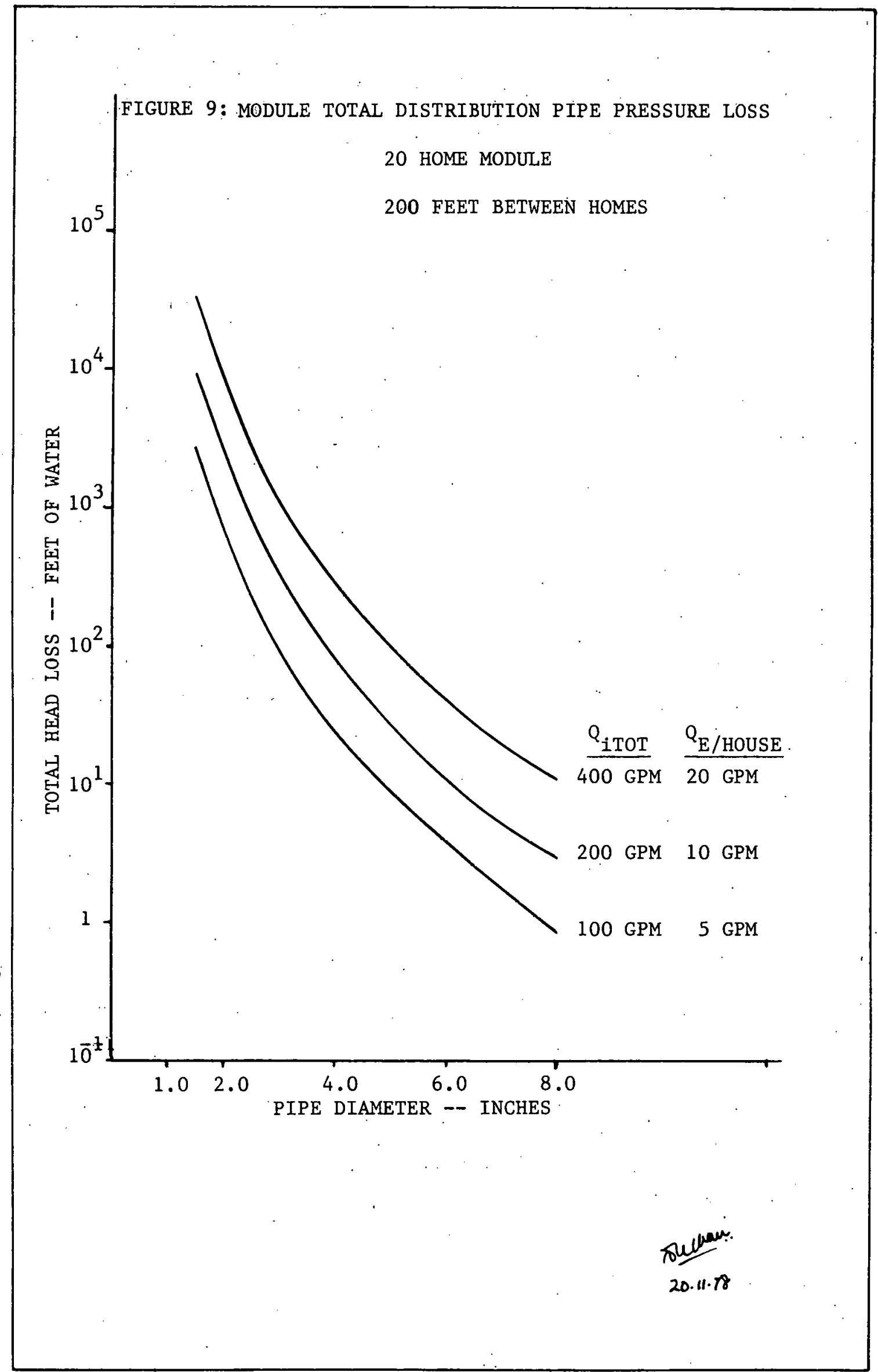


the pipe lines to buildings from the primary pipeline is minimized by using pipelines 1-inch or more in diameter as installation labor is a major cost.

If suitable aquifers are not in the immediate area of the module, primary lines can be constructed to nearby areas. For this case additional pumping power is required and performance is decreased.

Major buildings would be 1 or more independent modules. An ideal situation would be where an aquifer is situated under the building or under adjacent roads, parks, parking areas, etc. In this case pressure losses will be appreciably less than in the distributed home module.

All pipe utilized is Polyvinyl Chloride (PVC). The PVC pipe will:

1. Carry the pressure requirement,

2. Satisfy the temperature requirements,

3. Minimize pipe cost and installation cost, and

4. Eliminate the possibility of dissimilar metal to metal corrosion from galvanic reactions.

A more complete discussion is included in the analysis section IX.D. I.E. Additions of Ground Water, Waste Heat, and Solar Energy

In as much as heating and cooling loads will not match at most 1ocations, additional thermal energy must be added to or removed from the system annually. The work input for the cooling and heating must also be considered. An example of this equilibrium phenomenon follows:

Assume a 30,000 Btu annual cooling load and determine the energy available for heating. Assume coefficients of performance equal to 3.0 for both heating and cooling. This requires that 30,000 Btu must be removed from the controlled environment. The work required to remove the energy can be determined by the definition of coefficient of performance $(B)$ for cooling where $W$ is work input, $Q_{L}$ is the thermal energy removed and $\mathrm{Q}_{\mathrm{H}}$ is the thermal energy rejected or:

$$
B=3.0=\frac{Q_{L}}{W}=\frac{Q_{H}}{Q_{H}-Q_{L}}
$$


and the work is equal to:

$$
W=\frac{Q_{L}}{\beta}=\frac{30,000}{3.0}=10,000 \mathrm{Btu}
$$

The total thermal energy injected into the well is $\mathrm{Q}_{H}$ or $\mathrm{Q}_{\mathrm{L}}$ plus the work or $30,000+10,000$ which equals $40,000 \mathrm{Btu}$. For heating the $40,000 \mathrm{Btu}$ becomes $\mathrm{Q}_{\mathrm{L}}$ in the definition of coefficient of performance $\left(\beta^{\prime}\right)$ for heating or:

$$
\begin{array}{r}
\beta^{\prime}=3.0=\frac{Q_{H}}{Q_{H}-Q_{L}}=\frac{Q_{H}}{Q_{H}-40,000} \\
Q_{H}=\frac{Q_{L} \beta^{\prime}}{\beta^{\prime}-1}=40,000 \times \frac{3}{3-1}=60,000 \mathrm{Btu}
\end{array}
$$

This means $60,000 \mathrm{Btu}$ is available for heating in this case compared to the 30,000 Btu for cooling or a $2: 1$ ratio. This indicates that in an area where the heating load equals twice the cooling load, the energy storage system breaks even on cooling and heating if both COPs equal 3. Where the ratio of heating to cooling is greater than 2 , extra heating is required; and where the rat lo of heating to cooling is less than 2, extra cooling is required.

- The extra heat must be supplied by waste heat or low cost solar energy and the cooling by a cooling tower or aquifer drawdown. Controls to make the system operate must be developed. Analytically, the relationship between heating and cooling develops from an algebraic combination of coefficients of performance as follows:

$$
\frac{Q_{\text {Heating }}}{Q_{\text {Cooling }}}=\frac{\beta+1}{\beta} \times \frac{\beta^{\prime}}{\beta-1}
$$


The ratio of heating to cooling load is shown in Figure 10 as a function of the heating and cooling coefficients of performance.

In most of the country extra heat must: be added to the system. This is espectally true with some of the alternative systems which slow higher coefficients of performance.

In the South and Southwest and for major bulldings, supplementary cooling is required. The following are possible solutions:

1. Place a cooling tower in the system. Since annual storage exists, a small unit operating continuousfy can be utilized. In a major building, the untt is expected to be owner owned; and, in a residential system, the public utility is expected to own and maintain the units. This system is recommended for large building modules.

2. Place a heat exchanger or dry tower in the system. With annual storage the system can be operated during cold weather to provide heat release.

3. Discard water after heating. This method uses cold groundwater to replace the heated water which is then discarded. This is the system presently utilized in many water-source heat pump installations and is the system presently recommended for scattered modules.

In northern latitudes, large buildings (1arge internal loads) can be combined with residential and small commercial buildings to remove the excess heat. The totally residential modules will require heat addition.

The basic methods to add energy to systems requiring additional heat are: 


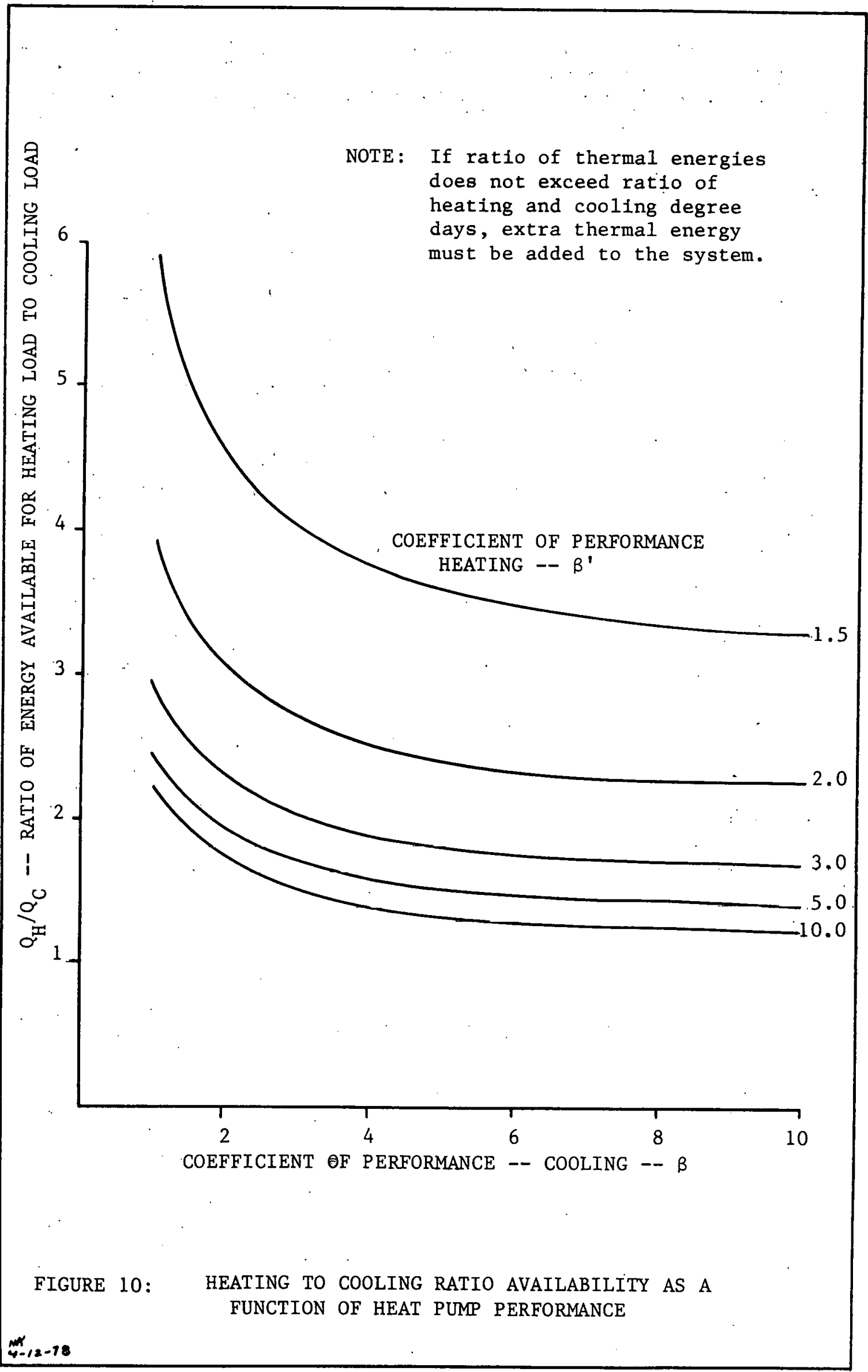


1. Waste Heat Sources -- This requires placing a heat exchanger between the source and the water distribution system. Any source such as power plant cooling water, waste heat from laundry runoff, etc., can be utilized. In the home the waste water from showers, sinks, aishwashers, washing machines, etc., can be run through a heat exchanger. When the temperature exceeds the required temperature (presently $80^{\circ} \mathrm{F}$ ), a control system will open a valve and pump water from the cold water line (well) to the warm water line (well) through the heat exchanger. These heat exchangers can operate on an annual basis with the available annual storage in the system. Energy discarded in the summer can be utilized in the winter.

2. Solar Energy -- Low temperature, low cost solar panels, primarily swimming pool panels, can be used for supplemental energy. Three types of solar systems are analyzed in section IX.E. These are a hard molded rubber solar panel, a solar panel made from polyethylene bags, and a solar panel system using polyethylene bags as shallow pond collectors. These systems can be used only during the summer months to preclude freezing problems. Controls would be similiar to the heat exchanger controls. When the temperature reaches the proper value, valves open and a pump transfers water from the cold line (well) to the warm line (well) through the solar panels. The utility company is expected to own and maintain these systems. It should be noted that. for roof installations ideally located buildings may be used. Each building does not need to have an individual system in a community integrated system.

3. Matching Buildings in Modules or Groups of Modules -- Large buildings normally have large internal heat loads and lower heat losses 
per square foot. For example, multistory buildings have no losses through ceilings and floors. By matching the modules with large buildings and modules with smaller buildings, the heating load and cooling load combinations can be matched.

In general, heat addition can be a combination of all 3 methods. The 6-inch lines are sufficiently large to allow transport of water. A set of controls will have to be designed to regulate the variations. These are expected to be implemented and change with time over a period of years.

\section{I.F. The Public Service Utility}

A utility is recommended to drill the wells, install pumping equipment, controls, primary piping, etc., and maintain the systems. The controlled environment area owner is expected to be responsible for secondary pipe lines from the main distribution system to the individual heat pump systems.

Charges for utility involvement should be on a first-rate basis for water, based on heating and cooling equipment capacity. Minimizing equipment capacity will encourage energy efficient buildings. It should be noted that monthly water charges are expected to be less than $20 \%$ of heating and cooling costs. Direct energy costs are still expected to encourage a conservative energy lifestyle. Installation and monitoring of energy meters are nonessential costs with little benefit except to the utility. Meter reading and installation are expected to cost more than total monthly charges. Rates from $\$ 2.00$ to $\$ 5.00$ per thousand square ft. of floor area per month seem to be reasonable charges which cover both power costs and servicing costs. Capital costs for the distribution system will be in the amount of $\$ 1,000$ per home and be on 
an assessment basis, such as water lines.

Service by the utility is expected to be minimal with less than 1 service call per pump well unit per year. If modules are joined, emergency type calls at odd hours should be almost nonexistent as adjacent modules will have short time (up to a few weeks) capability to provide water for an outage module. The proposed system eliminated the need for an onsite operator.

\section{I.G. Minor Related Systems}

The water system can supply large amounts of water for short period emergencies. Each module will have a water pumping capacity in excess of 400 gallons per minute (using warm and cold wells). A few interconnected modules can provide more than sufficient water for fire fighting units. In case of a major disaster.with complete loss of power, the use of auxiliary power to the module system can provide emergency water. I.H. Summary

The proposed heat pump centered integrated energy system combines businesses and neighborhoods into a module structure which provides warm and cold water through the use of thermal energy storage in aquifers for heat pumps at minimum capital costs. Annual thermal energy storage allows the excess (unwanted) energy in the summer to be stored for winter use using naturally available storage. 


\section{POTENTIAL APPLICATIONS}

The proposed system has applications for basically any heating and cooling system in the United States located near an aquifer with sufficient yield capacity. Over $60 \%$ of inhabited areas $f a l l$ in this category, excluding the Alaska permafrost area. Applications can include:

1. Residential neighborhoods,

2. Commercial buildings, multistory office buildings, etc.,

3. Apartment complexes, and

4. Various combinations of the above.

Limitations exist in 2.cases.

1. For small isolated loads, the cost of well drilling, pipeline distribution, and maintenance could make the system uneconomical; and

2. A major multistory building in a populated area might not have a sufficiently large aquifer for thermal storage to satisfy heating and cooling capacity of the building. Combination of this module with modules of other buildings or modules of a residential area can correct this situation. Also, auxiliary heating or waste heat and/or cooling tower can make up the difference in required aquifer capacity.

As demonstrated by the economic analysis for a 20-home module, the proposed system can be incorporated economically in essentially any new residential subdivision. The well system and distribution system can be incorporated in the capital price for the lots. Geographically the system is economically feasible nationwide in areas which have aquifers, with the exception of southern Florida. In this area the best system is probably a ground water system with 1 well and single piping for a neighborhood. 
Farther north, Alternative II is ideal for the required ratio of heating to cooling. This region has the largest peak power plant load during the summer. These peaks can be reduced by $60 \%$ if the total system is implemented. Cooling towers operating during winter months in these regions can provide energy equilibrium for the required Alternative II $45^{\circ} \mathrm{F}$-water.

As one moves farther north, extra heat is required in the system. Matching large buildings which have large internal loads with residential neighborhoods could balance the energy requirements. The second option is to use sources of available waste heat. The third option is to use low-cost swimming pool type solar energy collection during summer months, stored on an annual basis in an aquifer.

The system becomes much more feasible as gas, oil, and electric prices increase. Similar applicability is anticipated as gas and oil become scarce or rationed.

Conversion of large buildings, mostly with water source chillers, is the most likely application. The capital costs are minimized as most equipment can still be utilized. This is more feasible as interruptible gas service becomes more prevalent.

As central air conditioning is added in homes, presently less than $30 \%$ saturation, the capital cost of installing the systems is offset by the air conditioner cost. Social pressure for air conditioning can accelerate implementation of the proposed system.

The social pressure for solar energy can be an additional incentive. The system with solar supplemental energy is a solar-assisted heat pump system and may be eligible for income tax credits. Additional tax credits for implementation could accelerate the process. 
An economic incentive could be reduced electric rates (lower peak loads) for communities incorporating the system.

Figure 11 is a map of the continental United States showing adequate porous aquifers for the proposed system ${ }^{1}$. of major importance are the areas adjacent to main river basins which generally afford suitable aquifers. The Mississippi, Missouri, Ohio, and Allegheny are examples of such basins. Major population centers are located along these stream basins. In addition to the aquifer shown, some fractured and porous metamorphic and igneous rock will serve as adequate aquifers. Essentially over $60 \%$ of the continental United States land area and over $70 \%$ of the continental United States population has adequate aquifers available for operation of this system. . Population statistics for 1975 show that $155,021,000(75 \%)$ of the population is concentrated in metropolitan areas and $58,000,000(25 \%)$ in non-metropolitan areas ${ }^{2}$. From the map it is assumed $75 \%$ of the metropolitan areas and $60 \%$ of the nonmetropolitan areas can use the proposed system. Combining numbers, $71 \%$ of United States households can utilize the system. It is assumed $75 \%$ of all United States businesses can utilize the system. Total applications as of 1975 are $^{2}$ :

$71 \%$ of $72,500,000$ households $=51,475,000$ households

$75 \%$ of $13,900,000$ active businesses $=10,400,000$ businesses

Extrapolating these numbers to the year 2000, which anticipates a $40 \%$ increase, results in possible applications for the year 2000 as $72,000,000$ households $14,600,000$ businesses Arbitrarily using 20 households and 10 businesses per module results in the installation of $5,060,000$ modules. 


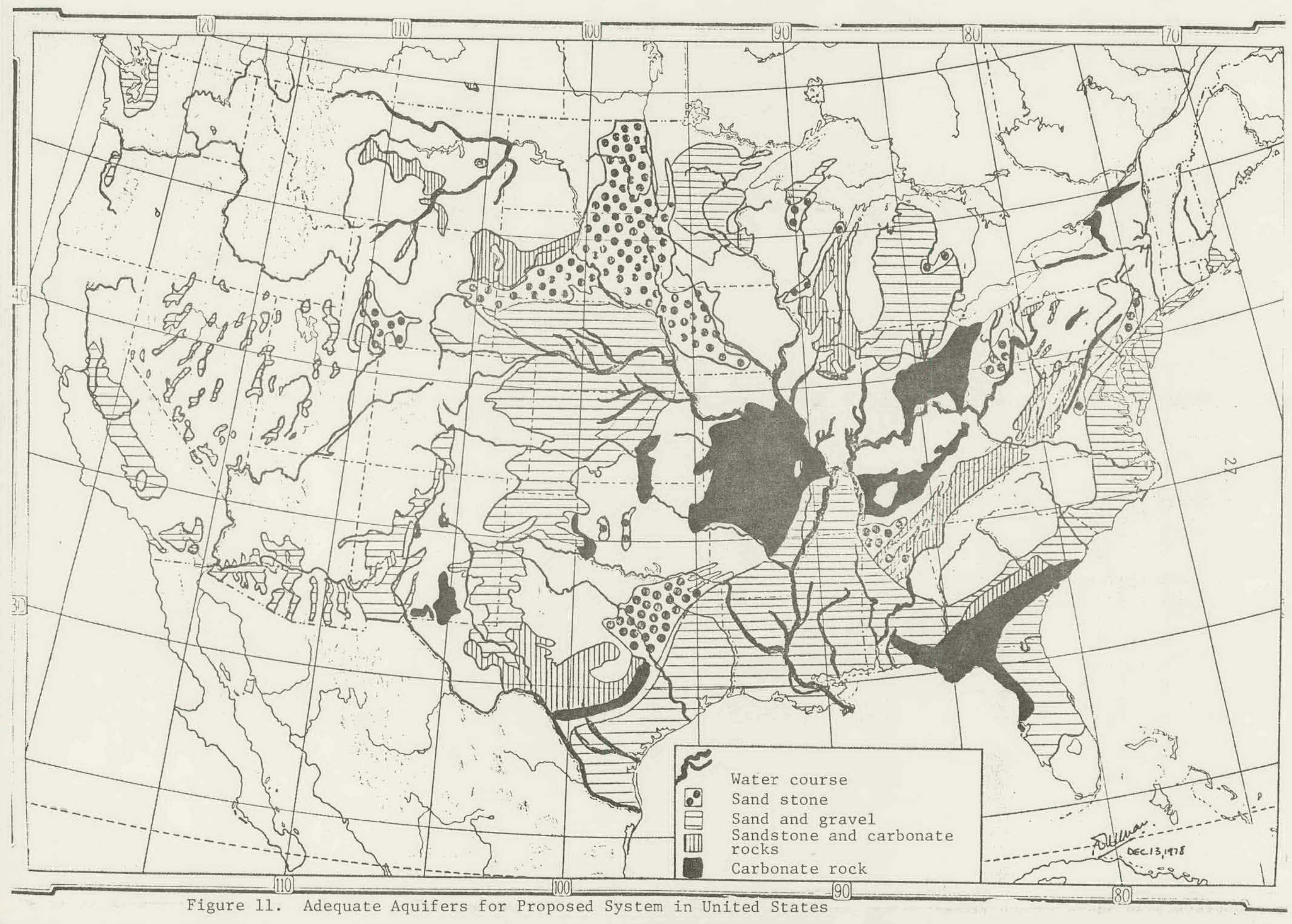


For air conditioning, use of electricity can be decreased by approximately $50 \%$ by using a combination of the proposed system and alternatives to replace present systems. For heating the use of natural gas (60\% in 1975) and fuel oil (22\% in 1975) is replaced by electricity which can be generated with less-critical fuels such as coal, hydropower, or nuclear energy ${ }^{2}$. For heating the energy requirement also decreased an average of $50 \%$ over systems presently utilized.

Energy usage per year in the United States is estimated as ${ }^{2}$ :

Total Energy Usage Quadrillion Btu

1975

2000

$$
70.6
$$

\begin{tabular}{c}
$\begin{array}{c}\text { Commercial and Residential Energy Usage } \\
\% \\
\text { Quadrillion Btu }\end{array}$ \\
\hline
\end{tabular}

17.8

12.5

Statistics for 1970 show that $70 \%$ of residential energy usage and $76 \%$ of commercial energy usage is for heating and cooling ${ }^{3}$. Applying an average $73 \%$ to the above numbers, energy usage in the United States for heating and cooling is:

$$
\begin{aligned}
& 19759.5 \text { quadrillion Btu } \\
& 2000 \quad 41.2 \text { quadrillion Btu }
\end{aligned}
$$

The proposed systems with the present state-of-the-art water source heat pumps can save $50 \%$ of the energy required in 2000 when compared to energy consumption rates of present day heating and cooling systems or:

\section{6 quadrillion Btu per yeàr}

which equals 10 million barrels of petroleum per day which equals 20.6 trillion $\mathrm{ft} .^{3}$ of natural gas per day which equals 824 million tons of coal per year

The proposed system and alternatives have the potential for economically feasible implementation with a corresponding potential to reduce energy consumption. 


\section{EXPECTED PERFORMANCE}

The expected performance of the proposed system and first alternative shows a major increase in performance with respect to energy usage. The second 2 alternatives show larger increases in performance. The performance of the proposed system is compared to present systems, including resistance heating, oil and gas heat, and air source heat pumps. The comparison is made in energy utilized, namely thermal energy at the furnace or thermal energy in the power plant generating electricity. The proposed system shows an advantage over all other systems.

An energy analysis of the proposed system and present heating and cooling systems is shown in Figure 12. The new development is the economical annual thermal energy storage provided by nature. The energy distribution system (water distribution) allows the feasibility of injecting waste heat or solar energy into the system at any time of the year. The power plant is included in the schematic as performance for all systems has a final comparison on the basis of paid-for-energy -fuel into the system.

A Second Law of Thermodynamics. Analysis indicates the effect of decreasing the temperature difference over which the heat pump operates. Efficiency in the universe is defined as minimizing the rate of entropy generation. Per Figure 13, the total entropy generation equals $(\mathrm{Q}+\mathrm{W}) / \mathrm{T}_{\mathrm{H}}-\mathrm{Q} / \mathrm{T}_{\mathrm{L}}$. This term must be greater than or equal to zero. As the temperature approach each other, the work required to keep $\Delta S$ positive approaches zero. This does not account for inefficiencies in the heat pumps, but places the heat pump in a system where performance can be maximized. The highest performance heat pumps available are used in the system to keep the work as close as possible to the minimum required work. 


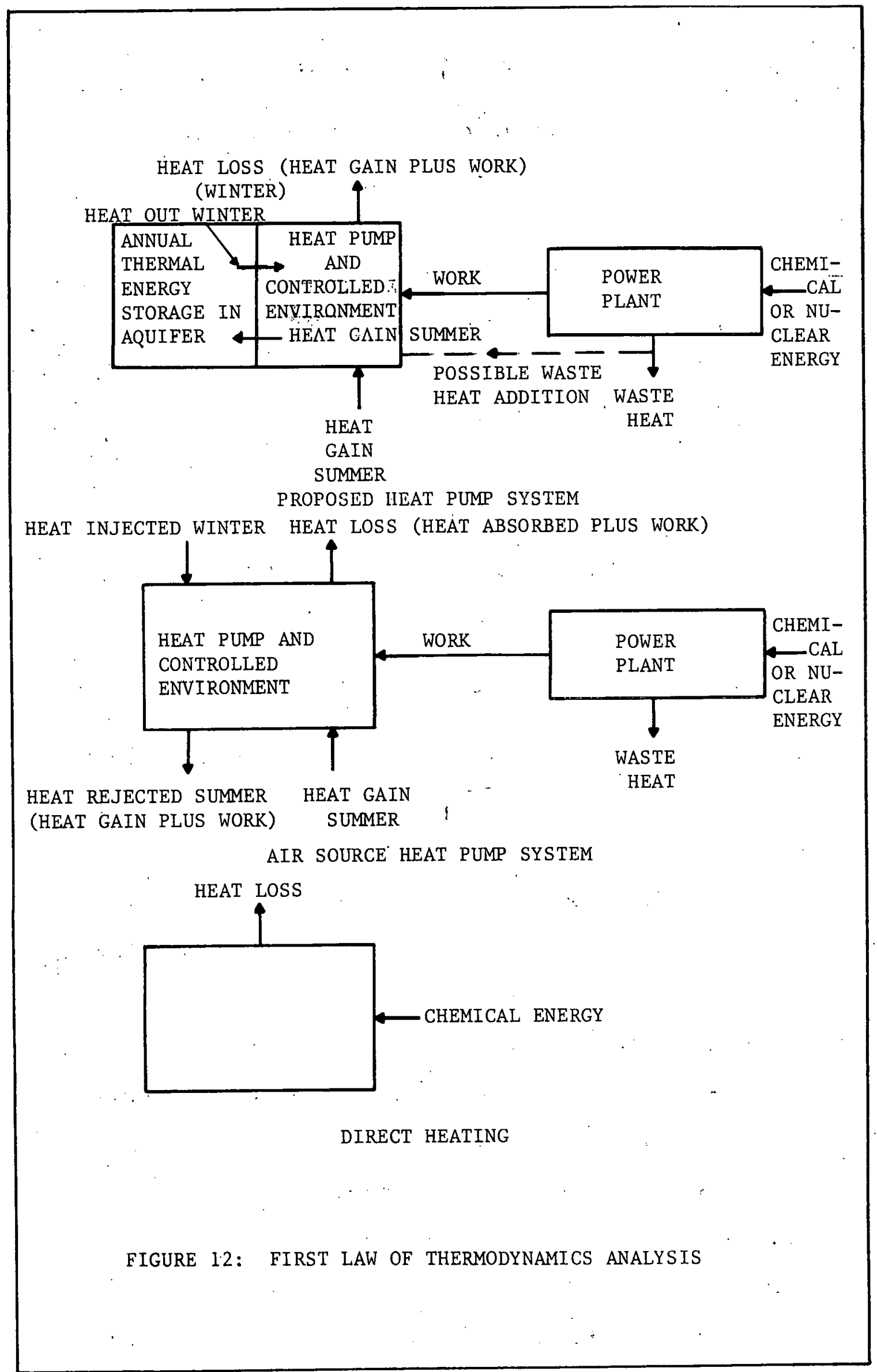


HIGH WIPERATURE ENERGY REJECTION $\mathrm{Q}_{\mathrm{H}} \mathrm{AT}$ TEMPERATURE $\mathrm{T}_{\mathrm{H}}$

HIGH TEMPERATURE ENERGY REJECTION

$\Delta S=\frac{Q_{H}}{T_{H}}=\frac{Q_{L}+W}{T_{H}}$

LOW TEMPERATURE ENERGY

ABSORPTION $Q_{\mathrm{I}}$ AT TEMPERATURE $\mathrm{T}_{\mathrm{L}}$

$\Delta \dot{S}=-\frac{Q_{L}}{T_{L}}$

WORK (W)

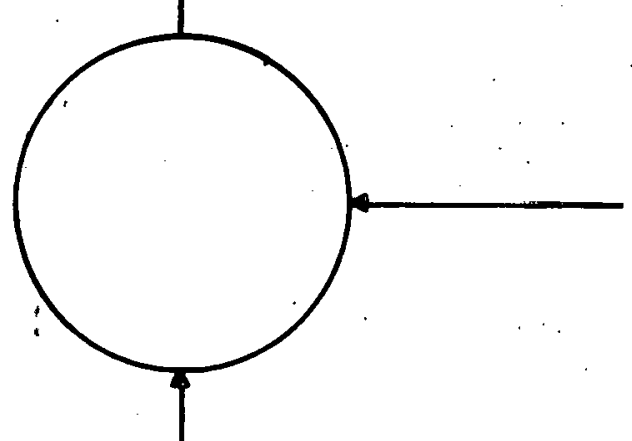

LOW TEMPERATURE ENERGY SOURCE AT TEMPERATURE $T_{L}$

FIG. 13 SECOND LAW OF THERMODYNAMICS ANALYSIS 
This project does not develop the heat pump but the system in which the heat pump operates. The Second Law of Thermodynamics Analysis indicates minimizing the temperature difference over which the heat pump operates is a major step towards maximizing performance.

Numerous studies have been made using the earth and other types of storage to minimize these temperature differences. ${ }^{6,7}$ This has not only been true in the United States, but also in Europe. 9 . In many of the systems, the heat transfer is by pipes in the earth. In this study nature is allowed to provide the heat transfer mechanism, porous underground aquifers through which water can flow to transfer the thermal energy.

The proposed systems are first evaluated by coefficient of performance (COP) which is defined as:

$$
\begin{aligned}
& { }^{\mathrm{COP}} \text { Cooling }=\frac{\text { Energy Removed from Controlled Environment }}{\text { Work Required to Remove Energy }} \\
& \dot{C O P}_{\text {Heating }}=\frac{\text { Energy Added to Controlled Environment }}{\text { Work Required to Add Energy }}
\end{aligned}
$$

These definitions are applied directly and independently to the heating and cooling sequence (Tables 1 to 4 ). In Alternatives II and III, where direct heat exchanger is used for cooling or heating, very high coefficients of performance result. These values are somewhat artifical as the opposite sequence, heating and cooling, provides the proper temperature water to allow the direct thermal energy transfer. Part of this energy could be charged to the direct heat transfer. As a result, the annual energy usage must be applied to evaluate these sys-. tems. The energy for heating and cooling must be properly balanced.

The performance values for the proposed system and alternatives should be appreciably higher in the near future. The water source heat pumps produced by American Air Filter from which basic performance data 
have been taken are somewhat obsolete. New compressors of higher efficiency have been on the market for a few years. Basic data from Tescumseh support this fact. ${ }^{4}$ In addition, motor efficiencies have improved and heat-exchanger areas have increased. American Air Filter is incorporating these changes (all components already on the market) in all of their units over..the next 2 years. . They estimate these changes will increase their water source heat pump performance by a minimum of 20 to 30 percent. This means the proposed system and alternatives will increase performance by the same magnitude. Air source heat pump A already has incorporated these changes and is utilized as a comparison with these systems. The best available heat pump data have been used for comparisons. The comparisons should indicate larger performance gains. Equipment in the proposed system has not been updated with components on the market, whereas the compared system performance already has incorporated these items. Very few of these high-performance heat pumps have been installed.

Performance of the proposed heat pump systems and alternative systems are shown in Tables 1 to 4 . The proposed system performance (Table 1) is that of a basic water source heat pump using 300 watts for water pumping power, $60^{\circ} \mathrm{F}$ water for cooling, and $80^{\circ} \mathrm{F}$ water for heating. Alternative $I$, Figure 14, uses a 50\% efficlency heat exchanger between the incoming air and water to transfer part of the energy by natural processes. A step gain is recorded in performance. Tables 3 and 4 use natural heat transfer for either cooling or heating. In Alternative II Table $365^{\circ} \mathrm{F}$ water is used for heating. Removing the energy from the water reduces water temperature to $45^{\circ} \mathrm{F}$. This water is then used as 
TABLE 1: PERFORMANCE OF WATER SOURCE HEAT PUMP $A^{*}$

IN PROPOSED SYSTEM

COOLING

\begin{tabular}{|c|c|c|c|c|c|c|c|}
\hline $\begin{array}{l}\text { ENTERING } \\
\text { WATER TEMP }{ }^{\circ} \mathrm{F}\end{array}$ & $\begin{array}{l}\text { ID } \\
\text { WBT } \\
\end{array}$ & $\begin{array}{c}\text { TOTAL CAP } \\
\text { MBTUH } \\
\end{array}$ & $\begin{array}{c}\text { SENS } \\
75 \\
\end{array}$ & $\begin{array}{l}E \text { CAP } \\
80\end{array}$ & @ $\begin{array}{l}\mathrm{IDBT}^{2} \\
85 \\
\end{array}$ & $\begin{array}{c}\text { POWER }^{3} \\
\mathrm{KW} \\
\end{array}$ & $\mathrm{COP}^{4}$ \\
\hline 65 & $\begin{array}{l}61 \\
64 \\
67 \\
70\end{array}$ & $\begin{array}{l}31.8 \\
34.1 \\
36.8 \\
37.9\end{array}$ & $\begin{array}{l}26.3 \\
24.4 \\
21.2 \\
18.0\end{array}$ & $\begin{array}{l}29.2 \\
28.6 \\
25.2 \\
21.4\end{array}$ & $\begin{array}{l}31.8 \\
31.7 \\
30.7 \\
25.5\end{array}$ & $\begin{array}{l}3.90 \\
3.99 \\
4.06 \\
4.14\end{array}$ & $\begin{array}{l}2.39 \\
2.50 \\
2.66 \\
2.68\end{array}$ \\
\hline 75 & $\begin{array}{l}61 \\
64 \\
67 \\
70\end{array}$ & $\begin{array}{l}30.9 \\
33.15 \\
35.7 \\
36.8\end{array}$ & $\begin{array}{l}25.5 \\
23.7 \\
20.6 \\
17.4\end{array}$ & $\begin{array}{l}28.4 \\
27.7 \\
24.5 \\
20.8\end{array}$ & $\begin{array}{l}30.9 \\
30.8 \\
29.8 \\
24.8\end{array}$ & $\begin{array}{l}4.05 \\
4.15 \\
4.22 \\
4.30\end{array}$ & $\begin{array}{l}2.24 \\
2.34 \\
2.48 \\
2.51\end{array}$ \\
\hline 85 & $\begin{array}{l}61 \\
64 \\
67 \\
70\end{array}$ & $\begin{array}{l}30.3 \\
32.5 \\
35.0 \\
36.1\end{array}$ & $\begin{array}{l}25.0 \\
23.2 \\
20.2 \\
17.1\end{array}$ & $\begin{array}{l}27.8 \\
27.2 \\
24.0 \\
20.4\end{array}$ & $\begin{array}{l}30.3 \\
30.2 \\
29.2 \\
24.3\end{array}$ & $\begin{array}{l}4.13 \\
4.23 \\
4.30 \\
4.38\end{array}$ & $\begin{array}{l}2.15 \\
2.25 \\
2.39 \\
2.42\end{array}$ \\
\hline
\end{tabular}

HEATING

\begin{tabular}{|c|c|c|c|c|c|c|c|}
\hline $\begin{array}{l}\text { ENTERING } \\
\text { WATER. TEMP }\end{array}$ & \multicolumn{4}{|c|}{ CAP @ IDBT ${ }^{\circ} \mathrm{F}$, MBTUH } & \multicolumn{2}{|r|}{ POWER } & $10^{\circ}$ \\
\hline & & & & & & & \\
\hline 60 & 44.5 & 43.7 & 42.8 & 41.9 & 41.5 & 4.36 & 2.88 \\
\hline 70 & 48.2 & 47.2 & 46.3 & 45.4 & 44.9 & 4.63 & 2.93 \\
\hline 80 & 54.1 & 53.0 & 52.0 & 50.9 & $50: 4$ & 5.02 & 3.04 \\
\hline 90 & 59.1 & 57.9 & 56.8 & 55.7 & 55.1 & 5.45 & 3.05 \\
\hline
\end{tabular}

1. Indoor Wet Bulb Temperature, ${ }^{\circ} \mathrm{F}$

2. Indoor Dry Bulb Temperature, ${ }^{\circ} F$

3. Power for compressor, fan, and water pump

4. $\mathrm{COP}=$ Capacity $(\mathrm{Kw}) / \mathrm{Kw}$ (compressor + fan +300 watts for water pumping)

* Based on manufacturer's data at flow rate of 6 GPM for American Air Filter model HW33, taken from manfacturer's catalog specification page ENER-1-118-NOV-01. Cost $\$ 1,300$ installed per J. E. Christian, Unitary Water to Air Heat Pumps, ANL/CES/TE77-9. 
TABLE 2: SYSTEM PERFORMANCE FOR MODIFIED WATER SOURCE HEAT PUNP A WITH PRE-WATER - TO AIR HEAT EXCHANGER - - ALTERNATIVE I

\section{HEATING}

Basic Water-Source Heat Pump System Water-Source Heat Pump with Water-Air Exchanger

\begin{tabular}{|ccccc|cccccc|}
\hline IDBT $^{3}$ & Cap. $_{\text {F }}^{2}$ & $\begin{array}{c}\text { Input } \\
\text { Power } \\
\text { MBTUH }\end{array}$ & $\begin{array}{c}\text { KW } \\
\text { COP }\end{array}$ & $\begin{array}{c}\text { Water } \\
\text { Flow } \\
\text { GPM }\end{array}$ & $\begin{array}{l}\text { Pump } \\
\text { Cap. } \\
\text { MBTUH }\end{array}$ & $\begin{array}{c}\text { Heat } \\
\text { Exchanger } \\
\text { Cap. } \\
\text { MBTUH }\end{array}$ & $\begin{array}{c}\text { Total } \\
\text { Cap. } \\
\text { MBTUH }\end{array}$ & $\begin{array}{c}\text { Input } \\
\text { Power } \\
\text { KW }\end{array}$ & COP $^{4}$ & $\begin{array}{c}\text { Water } \\
\text { Flow } \\
\text { GPM }\end{array}$ \\
\hline 60 & 53.6 & 5.44 & 2.89 & 4 & 46.3 & 14.0 & 59.3 & 5.44 & 3.19 & 4 \\
65 & 52.6 & 5.44 & 2.83 & 4 & 48.0 & 10.4 & 58.4 & 5.44 & 3.15 & 4 \\
70 & 51.6 & 5.44 & 2.78 & 4 & 49.2 & 6.9 & 56.1 & 5.44 & 3.02 & 4 \\
\hline
\end{tabular}

\section{COOLING}

Basic Water-Source Heat Pump System Water-Source Heat Pump With Water-Air Exchanger

\begin{tabular}{|ccccc|cccccc|}
\hline IDBT $_{\text {F }}^{3}$ & $\begin{array}{c}\text { Cap. }^{2} \\
\text { MBTUH }\end{array}$ & $\begin{array}{c}\text { Input } \\
\text { Power } \\
\text { KW }\end{array}$ & COP & $\begin{array}{c}\text { Water } \\
\text { Flow } \\
\text { GPM }\end{array}$ & $\begin{array}{l}\text { Pump } \\
\text { Cap. } \\
\text { MBTUH }\end{array}$ & $\begin{array}{c}\text { Exchanger } \\
\text { Cap. } \\
\text { MBTUH }\end{array}$ & $\begin{array}{c}\text { Total } \\
\text { Cap. } \\
\text { MBTUH }\end{array}$ & $\begin{array}{c}\text { Input } \\
\text { Power } \\
\text { KW }\end{array}$ & COP $^{4}$ & $\begin{array}{c}\text { W1ow } \\
\text { GPM }\end{array}$ \\
\hline 70 & 37.1 & 3.98 & 2.73 & 5 & 37.5 & 6.9 & 44.4 & 3.98 & 3.27 & 6 \\
75 & 37.1 & 3.98 & 2.73 & 5 & 37.1 & 10.4 & 47.5 & 3.98 & 3.50 & 6 \\
80 & 37.1 & 3.98 & 2.73 & 5 & 36.4 & 13.8 & 50.2 & 4.10 & 3.59 & 6 \\
85 & 37.1 & 3.98 & 2.73 & 5 & 36.0 & 17.3 & 53.3 & 4.20 & 3.72 & 6 \\
\hline
\end{tabular}

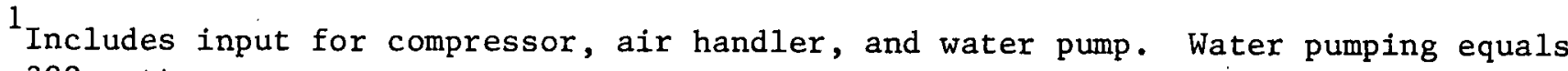
300 watts.

2 Total capacity (dry coil). Calculations based on survey of manufacturer's data at inlet water temperatures of $60^{\circ} \mathrm{F}$ for cooling and $80^{\circ} \mathrm{F}$ for heating.

${ }^{3}$ Indoor Dry Bulb Temperature.

4

Coefficient of Performance $=\frac{\text { Capacity, MBTUH/3.412 MBTU/KW }}{\text { Power Input }}$

* Based on manufacturer's data for American Air Filter model HW33, specification page ENER-1-118 
TABLE 3: SYSTEM PERFORMANCE FOR MODIFIED WATER SOURCE HEAT PUMP A WITII PRE-WATER TO AIR HEAT EXCHANGER - ALI'ERNATIVE II

Water Temperature Variation $40^{\circ} \mathrm{F}$ to $60^{\circ} \mathrm{F}$

HEATING

\begin{tabular}{|c|c|c|c|c|c|}
\hline $\begin{array}{l}\text { IDBT }^{1} \\
\left({ }^{\circ} \mathrm{F}\right)\end{array}$ & $\begin{array}{l}\text { A IRFLOW } \\
\text { (SCFM) }\end{array}$ & $\begin{array}{l}\text { WATERFLOW } \\
\text { (GPM) }\end{array}$ & $\begin{array}{l}\text { CAPACITY } \\
\text { (MBTUH) }\end{array}$ & $\begin{array}{l}\text { POWER }^{2} \\
(\mathrm{KW})\end{array}$ & $\mathrm{COP}^{3}$ \\
\hline $\begin{array}{l}60 \\
65 \\
70\end{array}$ & $\begin{array}{l}1000 \\
1000 \\
1000\end{array}$ & $\begin{array}{l}4.1 \\
4.0 \\
3.9\end{array}$ & $\begin{array}{l}40.6 \\
39.0 \\
38.6\end{array}$ & $\begin{array}{l}4.36 \\
4.36 \\
4.36\end{array}$ & $\begin{array}{l}2.73 \\
2.62 \\
2.59\end{array}$ \\
\hline \multicolumn{6}{|c|}{ COOLING } \\
\hline $\begin{array}{l}\text { IWBT }^{4} \\
\left({ }^{\circ} \mathrm{F}\right)\end{array}$ & $\begin{array}{l}\text { AIRFLOW } \\
\text { (SCFM) }\end{array}$ & $\begin{array}{l}\text { WATERFLOW } \\
\text { (GPM) }\end{array}$ & $\begin{array}{l}\text { CAPACITY } \\
\text { (MBTUH) }\end{array}$ & $\begin{array}{l}\text { POWER } \\
\text { (WATTS) }\end{array}$ & $\cdot \mathrm{COP}^{3}$ \\
\hline $\begin{array}{l}61 \\
64 \\
67 \\
70\end{array}$ & $\begin{array}{l}1000 \\
1000 \\
1000 \\
1000\end{array}$ & $\begin{array}{l}2.4 \\
3.9 \\
4.9 \\
6.0\end{array}$ & $\begin{array}{l}20.2 \\
35.1 \\
45.3 \\
56.4\end{array}$ & $\begin{array}{l}900 \\
900 \\
900 \\
900\end{array}$ & $\begin{array}{r}6.52 \\
11.04 \\
14.08 \\
18.04\end{array}$ \\
\hline
\end{tabular}

1. Indoor Dry Bulb Temperature, ${ }^{\circ} \mathrm{F}$

2. Power Required for Compressor, Fan, and Water Pumping

3. $\operatorname{COP}=\underset{(\mathrm{MBTUH})}{\mathrm{CAPACITY}} /(\operatorname{Power}(\mathrm{KW}) \times 3.412(\mathrm{MBTUH} / \mathrm{KW}))$

4. Indoor Wet Bulb Temperature At $70^{\circ} \mathrm{F}$ Dry Bulb Temperature

5. Net Capacity $=$ Total Capacity - Fan Input $(600 \mathrm{~W}=2.05$ MBTUH $)$

6. Fan $0.6 \mathrm{KW}$, Pumping $0.3 \mathrm{KW}$

Heat Pump Data Extracted From American Air Filter Specification Sheet ENER - 1 - 118 - NOV. - 01 For HW-33 Unit 
TABLE 4: SYSTEM PERFORMANCE FOR MODIFIED WATER SOURCE HEAT PUMP A. WITH PRE-WATER TO AIR HEAT EXCHANGER - ALTERNATIVE III

Water Temperature Variation $80^{\circ} \mathrm{F}$ to $100^{\circ} \mathrm{F}$

HEATING

\begin{tabular}{|c|c|}
\hline 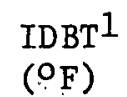 & $\begin{array}{l}\text { AIRFLOW } \\
\text { (SCFM) }\end{array}$ \\
\hline
\end{tabular}

602000

$65 \quad 2000$

$70 \quad 2000$

$\begin{array}{ll}\operatorname{IWBT}^{5} & \text { AIRFLOW } \\ \left({ }^{\circ} \mathrm{F}\right) & (\mathrm{SCFM})\end{array}$

$61 \quad 1000$.

$64 \quad .1000$

$67 \quad 1000$

70

1000

WATERFLOW
(GPM)

4.8

4.2

3.6

4.5

4.6

4.8

5.0

$$
\begin{aligned}
& \text { CAPACITY }^{2} \\
& \text { (MBTUH) }
\end{aligned}
$$

51.6

45.7

39.7

COOLING

$\begin{array}{ll}\text { WATERFLOW } & \text { CAPACITY } \\ \text { (GPM) } & \text { (MBTUH) }\end{array}$

29.7

31.8

34.3

35.4
POWER
(KW)

1.05

1.05

1.05

INPUT $^{3}$

(KW)

4. 21

4.31

4.38

4.46 $\operatorname{cop}^{4}$

10.01 .

8.93

7.76

$\operatorname{cop}^{4}$

2.07

2.17

2.30

2.32

1. Indoor Dry Bulb Temperature, ${ }^{\circ} \mathrm{F}$

2. Net Capacity, Heat Exchanger + Fan Input

3. Total Power For Fan, Pump Work and Compressor

4. $\operatorname{COP}=\underset{(\mathrm{NBT} \text { CAPACITY }}{\text { NBTH }} /($ Power $(\mathrm{KW}) \times 3.412(\mathrm{MBTUH} / \mathrm{KW}))$

5. Indoor Wet Bult Temperature

6. Fan 1.2KW, Pumping $0.3 \mathrm{KW}$

Heat Pump Data Extracted From American Air Filter Specification Sheet ENER - 1 - 118 - NOV. - 01 For HW33 Unit With Twin Blower Unit For Heating 


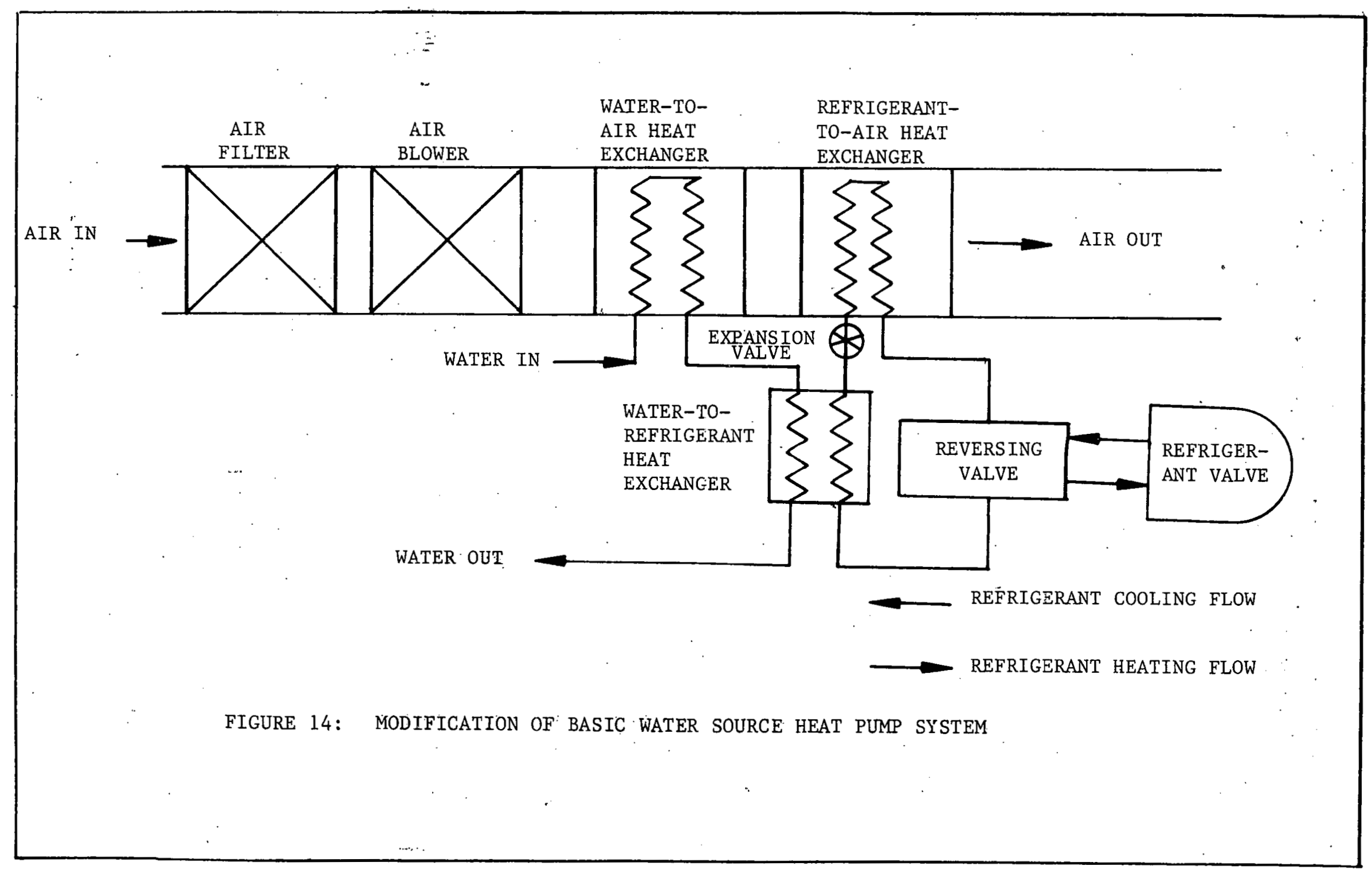


direct cooling for air conditioning. As a result, the heating COP decreases but the cooling COP shows a very large increase. The inverse occurs for Alternative III (Table 4). Water at $80^{\circ} \mathrm{F}$ is used for cooling which results in heating the water to $100^{\circ} \mathrm{F}$. The $100^{\circ} \mathrm{F}$ water is then used to heat directly. The cooling COP decreases; however, the heating COP shows a large increase. The increase is not as large as for natural cooling as an increase in air flow is required. No change in water flow rate is necessary.

Figures 15 and 16 show a major advantage of the system performance versus the air source heat pumps performance. Neither the coefficient of performance nor the capacity are effected by ambient conditions. The air source heat pumps at $0^{\circ} \mathrm{F}$ have 1 ost $80 \%$ of their $60^{\circ} \mathrm{F}$ capacity and $60 \%$ of their performance.

A major benefit is a reduction in power plant peak loads. Using Alternative II in parts of the South and the East Coast could eliminate peak summer loads. Matching loads to system alternatives, to types of buildings, and to power supplies is a performance possibility: The ratio of peak loads with Alternative II to Air Source Heat Pump C for air conditioning is proportional to the ratio of COP's or a decrease of over $60 \%$. Energy comparisons show a load decrease of $80 \%$. Integrating some of these systems in power plant operation decreases peak load.

A second major benefit is that oil and gas are not necessary. The power plants can operate on coal or nuclear energy saving critical fuels.

Tables 5 and 6 present the heating and cooling energy requirements for the various type systems for the well-insulated typical home 1isted in Section IX.A. for 5 cities. This home is an energy-conservation home and uses much less energy than over $99 \%$ of the present homes. A 


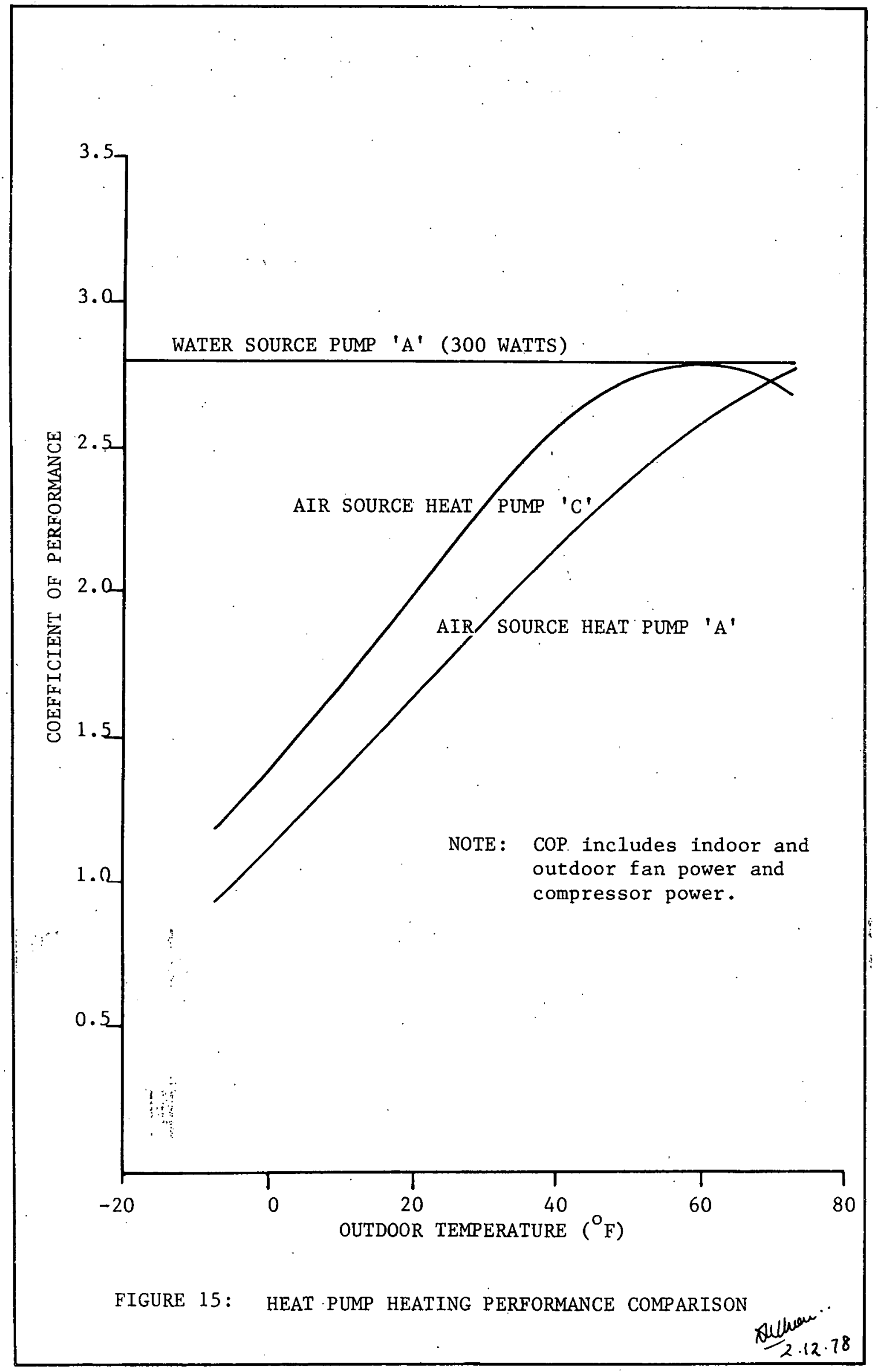




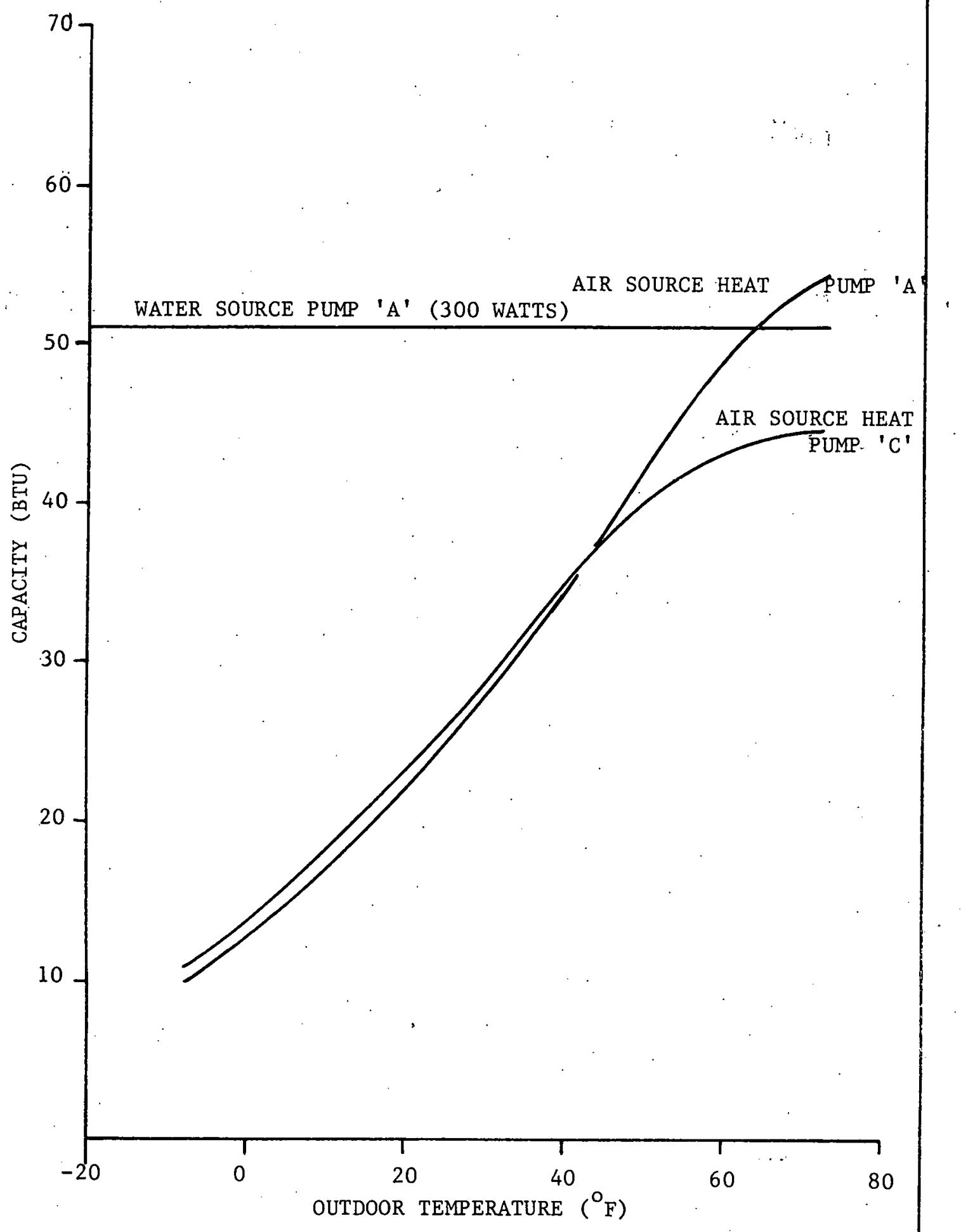

FIGURE 16: HEAT PUMP HEATING CAPACITY COMPARISON 
Table 5: Energy Requirements for Heating Well Insulated Typical Home Units

BIRMINGHAM NEW YORK MIAMI DETROIT ST. LOUIS

Annual Heating Load
Annual Heating Load

$\mathrm{Kw}-\mathrm{hr}$

$$
\begin{array}{ll}
11.3\left(10^{3}\right) & 14.8\left(10^{3}\right) \\
38.55\left(10^{6}\right) & 50.5\left(10^{6}\right)
\end{array}
$$

547
$18.7\left(10^{5}\right)$

$19 \cdot 5\left(10^{3}\right)$
$66.5\left(10^{6}\right)$

$14.7\left(10^{3}\right)$
$50.2\left(10^{6}\right)$

Direct Electrical Heating

Resistance Heating
Power Plant Input

Kw-hr
Btu

$14.8\left(1 q^{3}\right) \quad 14.8\left(1 q^{3}\right)$

$110\left(10^{6}\right)$

$144\left(10^{6}\right)$

547

Gas and 0il Heating

Furnace Input ${ }^{3}$

Gas

Oil

COP

Power Input

Power Plant Input ${ }^{2} \quad \begin{aligned} & \mathrm{Kw}-\mathrm{h} \\ & \mathrm{Btu}\end{aligned}$

COP

Power Input
Power Plant Input

COP

Power Input

Power Plant Input ${ }^{2} \quad \begin{aligned} & \mathrm{Kw}-\mathrm{h} \\ & \mathrm{Btu}\end{aligned}$

Air Source Heat Pump C

\section{$2.0 \quad 1.92$}

$\mathrm{Kw}-\mathrm{hr}$

$5.65\left(10_{6}^{3}\right)$

$7.71\left(10^{3}\right)$

Btu $55.1\left(10^{6}\right)$

$75.5\left(10^{6}\right)$

$53.4\left(10^{5}\right)$

$19.5\left(10^{3}\right)$
$190\left(10^{6}\right)$

$14.7\left(1 q^{3}\right)$

$143\left(10^{6}\right)$

Air Source Twin Heat Pump A

$\begin{array}{llllll}-- & 2.45 & 2.37 & 2.70 & 2.21 & 2.25 \\ \text { Kw-hr } & 4.61\left(10^{3}\right) & 6.24\left(10^{3}\right) & 203 & 8.82\left(10^{3}\right) & 6.53\left(10^{3}\right) \\ \text { Btu } & 44.9\left(10^{6}\right) & 50.9\left(10^{6}\right) & 1.98\left(10^{6}\right) & 86\left(10^{6}\right) & 53.7\left(10^{6}\right)\end{array}$

2.70

203

$30.2\left(10^{5}\right)$

107. $\left(10^{6}\right)$

1070

738

$81.0\left(10^{6}\right)$

810

559

20.8

$1.98\left(10^{6}\right)$

2.16

2.27

241

2. $35\left(10^{6}\right)$

$9.03\left(10^{3}\right)$

2.20

$6.68\left(10_{6}^{3}\right)$

$88\left(10^{6}\right)$

$65.2\left(10^{6}\right)$

1.76

$11.1\left(1 g^{3}\right)$

1.80

$108\left(10^{6}\right)$

$8.17\left(10^{3}\right)$
$79.7\left(10^{6}\right)$

Water Source Heat Pump A

COP

Power Input

2.78

--
$\mathrm{Kw}-\mathrm{hr}$
Btu

\subsection{8}

2.78

Power Plant Input ${ }^{2}$ Btu

$4.1\left(10^{3}\right)$

$5.32\left(10_{6}^{3}\right) \quad 197$

Modified Water Source Heat Pump A With Pre-Water Heat Exchanger -- Alternative I

COP

$\begin{array}{ll}\text { Power Input } & \mathrm{Kw}-\mathrm{h} \\ \text { Power Plant Input } & \mathrm{Btu}\end{array}$
3.02
$3.74\left(10^{3}\right)$
$36.5\left(10^{6}\right)$
3.023 .02

$4.90\left(10_{6}^{3}\right) \quad 181$

$47.8\left(10^{6}\right)$

$1.77\left(10^{6}\right)$

3.02

$6.46\left(10^{3}\right)$
$62.9\left(10^{6}\right)$
3.02

$4.87\left(10^{3}\right)$
$47.5\left(10^{6}\right)$

Modified Water Source Heat Pump A With Pre-Water Heat Exchanger -- Alternative II

COP

Power Input

Power Plant Input ${ }^{2} \quad \mathrm{Kw}-\mathrm{h}$
2.59
$4.36\left(10^{3}\right)$
$4.25\left(10^{3}\right)$

$4.36\left(10^{3}\right)$
$4.25\left(10^{7}\right)$
2.59

$5.7\left(10^{3}\right)$
2.59
$2.11\left(10_{7}^{2}\right)$
2.59

$7.53\left(10_{7}^{3}\right)$
2.59

$5.68\left(10_{7}^{3}\right)$

Modified Water Source Heat Pump A With Pre-Water Heat Exchanger -- Alternative III

COP

$\begin{array}{ll}\text { Power Input } & \mathrm{Kw}-\mathrm{h} \\ \text { Power Plant Input } & \mathrm{Btu}\end{array}$

\subsection{6}

7.76
$1.46\left(10^{3}\right)$
$1.42\left(10^{3}\right)$

7.76
$1.9\left(10^{3}\right)$
$1.86\left(10^{7}\right)$

7.76

70.49

$6.89\left(10^{5}\right)$

7.76

$2.51\left(10^{3}\right)$

7.76

2. $45\left(10^{7}\right)$

$1.89\left(10^{3}\right)$
$1.85\left(10^{7}\right)$

1. $\mathrm{COP}=1.0$

2. Combined Power Plant and Distribution Efficiency Equals 0.35 Based on Higher Heating Value of Fuel

3. Combined Furnace and Combustion Efficiency Equals 0.62 Based on Higher Heating Value of Fuel 
Table 6: Energy Requirements for Cooling Well Insulated Typlcal Home

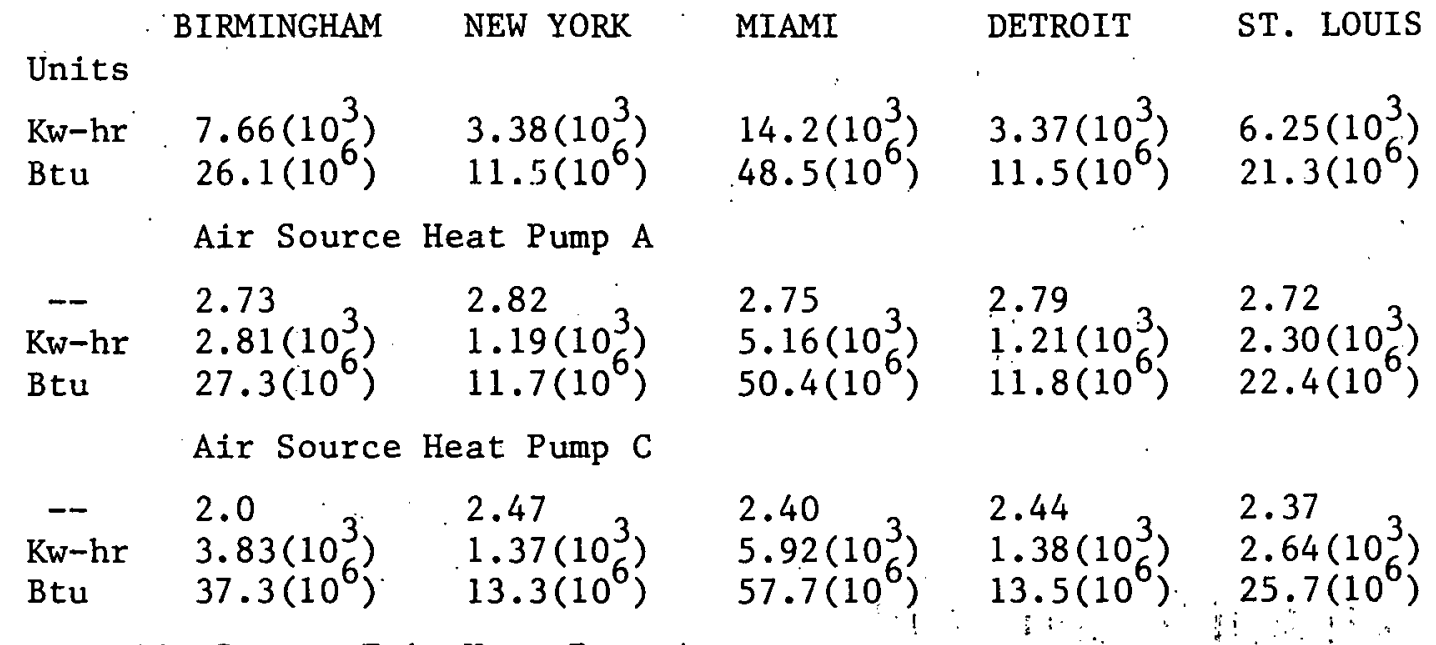

\section{Units}

\section{$\begin{array}{ll}7.66\left(10^{3}\right) & 3.38\left(10^{3}\right) \\ 26.1\left(10^{6}\right) & 11.5\left(10^{6}\right)\end{array}$ \\ Air Source Heat Pump A}

\subsection{9}

\section{Air Source Heat Pump C}

Ännual Cooling Load $\mathrm{Kw}-\mathrm{hr}$ Annual Cooling Load Btu

COP

$\begin{array}{ll}\text { Power Input } & \mathrm{Kw}-\mathrm{h} \\ \text { Power Plant Input } & \mathrm{Btu}\end{array}$

COP

Power Input

Power Plant Input ${ }^{2}$

COP

\begin{tabular}{|c|c|c|c|c|c|c|c|c|}
\hline $\begin{array}{l}\text { OP } \\
\text { Ower } \\
\text { Ower }\end{array}$ & $\begin{array}{l}\text { Input } \\
\text { Plant }\end{array}$ & Input $^{2}$ & $\begin{array}{l}-- \\
\mathrm{Kw}-\mathrm{hr} \\
\text { Btu }\end{array}$ & $\begin{array}{l}3.27 \\
2.34\left(10^{3}\right) \\
22.8\left(10^{6}\right)\end{array}$ & $\begin{array}{l}3.27 \\
1.03\left(10^{3}\right) \\
10.0\left(10^{6}\right)\end{array}$ & $\begin{array}{l}3.27 \\
4.34\left(10^{3}\right) \\
42.4\left(10^{6}\right)\end{array}$ & $\begin{array}{l}3.27 \\
1.03\left(10^{3}\right) \\
10.0\left(10^{6}\right)\end{array}$ & $\begin{array}{l}3.27 \\
1.91\left(10^{3}\right) \\
18.6\left(10^{6}\right)\end{array}$ \\
\hline
\end{tabular}

Power-Input

Power Plant Input ${ }^{2}$
Air Source Twin Heat Pump A

$\begin{array}{lll}-- & 2.72 & 2.81 \\ \text { Kw-hr } & 2.82\left(10^{3}\right) & 1.20\left(10^{3}\right) \\ \text { Btu } & 27.4\left(10^{6}\right) & 11.7\left(10^{6}\right) \\ & \text { Water Source Heat Pump A }\end{array}$

\section{COP}

Power Input
Power Plant Input

\section{$\begin{array}{ll}-- & 2.73 \\ \text { Kw-hr } & 2.81(10\end{array}$}

Kw-hr $27.3\left(10^{6}\right)$
2.73
$1.24\left(10^{3}\right)$
2.75

$5.16\left(10^{3}\right)$

Modified Water Source Heat Pump A With Pre-Water Heat Exchanger -- Alternative I

Modified Water Source Heat Pump A With Pre-Water Heat Exchanger -- Alternative II

COP

$\begin{array}{ll}\text { Power Input } & \mathrm{Kw}-\mathrm{h} \\ \text { Power Plant Input } & \mathrm{Btu}\end{array}$
$14.8 \quad 14.8$

Modified Water Source Heat
COP
Power Input

$\begin{array}{ll}\text { COP } & -- \\ \text { Power Input } & \mathrm{Kw}-\mathrm{hr} \\ \text { Power Plant Input } & \mathrm{Btu}\end{array}$
$14.8 \quad 14.8$

14.8
$9.59\left(10^{2}\right)$
$9.36\left(10^{6}\right)$
14.8

$2.28\left(10^{2}\right)$
$2.22\left(10^{6}\right)$
2.72

$2.31\left(10^{3}\right)$

1. $\mathrm{COP}=1.0$

2. Combined Power Plant and Distribution Efficiency Equals 0.35 Based On Higher Heating Value of Fuel

3. Combined Furnace and Combustion Efficiency Equals 0.62 BAsed On Higher Heating Value of Fuel 
3-ton heat pump capacity is basicalły required for either heating or cooling. The tables show the total energy in electrical units where applicable or as fuel heating values. for furnaces. Also, the electrical units are changed to total heating values for fuel entering the power plant. This allows total energy comparisons.

Tables 7 to 18 give heating and cooling energy input comparisons of the proposed system and three alternatives for present day systems for heating, cooling, and heating and cooling. These are basic energy inputs, fuel at the furnace or power plant. The values are for the wellinsulated typical home.

The comparisons indicate that in areas such as Miami with both mild, cold, and warm temperatures, the advantages of the system are questionable. Maximum performance is obtained with Alternative $I$ in the area from central Florida to northern Georgia, Alabama, and Mississippi westward. Farther north Alternative II gives the best performance until heating loads overshadow cooling loads in the range of a ratio of $3: 6$. For example, Detroit shows approximately the same performance for Alternative I and II. Farther north Alternative III is most feasible if plus $100^{\circ} \mathrm{F}$ waste heat is available. Otherwise. Alternative I shows better results.

For the vast majority of the country, with the exception of the lower one-half of Florida and part of the western coastal area, the system shows a sizable increase in performance. 
Table 7: Energy*Comparison for Heating and Cooling Well Insulated Typical Home Percentage Savings Utilizing Proposed Water Source Heat Pump A

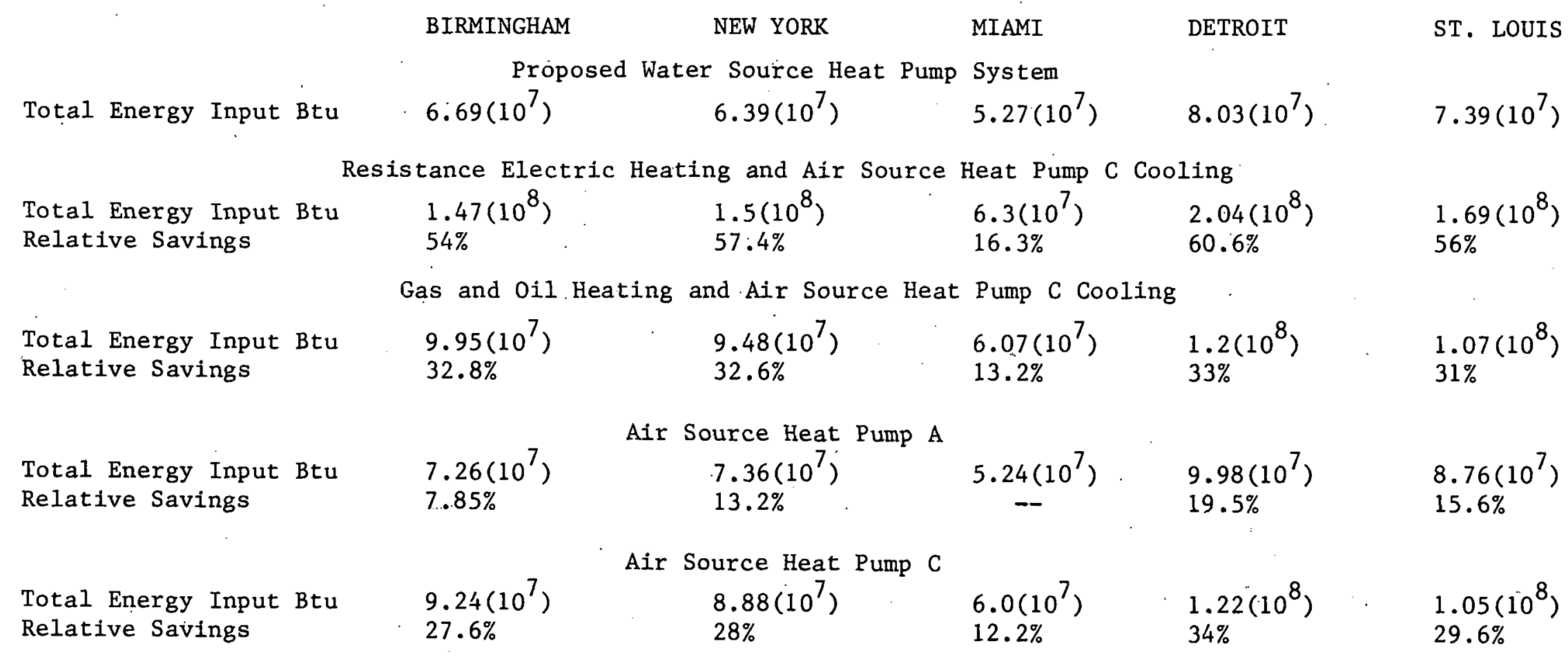

*Energy represents energy input at power plant for electrical power plus higher heating value of fuel for furnace input. 
Table 8: Energy*Comparison for Heating Well Insulated Typical Home Percentage Savings Utilizing Proposed Water Source Heat Pump A
BIRMINGHAM
NEW , YORK
MIAMI
DETROIT
ST.LOUIS

Proposed Water Source Heat Pump System

Total Energy Input Btu

Total Energy Input Btu Relative Savings

Total Energy Input Btu Relative Savings

Total Energy Input Btu Relative Savings

Total Energy Input Btu Relative Savings

$39.6\left(10^{6}\right)$

$51.9\left(10^{6}\right)$

$1.92\left(10^{6}\right)$

$68 \cdot 3\left(10^{6}\right)$

$51.6\left(10^{6}\right)$

Electric Resistance Heating

$110\left(10^{6}\right)$
$64 \%$

$62.2\left(10^{6}\right)$ $36 \%$

$45 \cdot 3\left(10^{6}\right)$ $12.6 \%$

$55.1\left(10^{6}\right)$

$28.1 \%$

$$
144\left(10^{6}\right)
$$$$
64 \%
$$

$53.4\left(10^{5}\right)$

$64 \%$

Gas and Oil Heating

$$
81.5\left(10^{6}\right)
$$

$36 \%$

$30.2\left(10^{5}\right)$

$36.4 \%$

Air Source Heat Pump A

$61.9\left(10^{6}\right)$

$16 \%$

$1.98\left(10^{6}\right)$

$3 \%$

Air Source Heat Pump C $75.5\left(10^{6}\right)$ $31 \%$ $2.35\left(10^{6}\right)$ $18 \%$ $190\left(10^{6}\right)$

$64 \%$

$143\left(10^{6}\right)$ $64 \%$

$$
81.0\left(10^{6}\right)
$$$$
36 \%
$$

*Energy represents energy input at power plant for electrical power plus higher heating value of fuel for furnace input. 
Table 9: Energy* Comparison for Cooling Well Insulated Typical Home Percentage Savings Utilizing Proposed Water Source Heat Pump A
BIRMINGHAM
NEW YORK
MIAMI
DETROIT
ST. LOUIS

Proposed Water Source Heat Pump System

Total Energy Input Btu $27.3\left(10^{6}\right)$

$12.0\left(10^{6}\right)$

$50.8\left(10^{6}\right)$

$12.0\left(10^{6}\right)$

$22.3\left(10^{6}\right)$

Total Energy Input Btu Realtive Savings

$27.3\left(10^{6}\right)$

$--$

$$
37.3\left(10^{6}\right)
$$

$26.8 \%$

Total Energy Input Btu Relative Savings
Air Source Heat Pump A

$$
11.7\left(10^{6}\right)
$$$$
50.4\left(10^{6}\right)
$$$$
\text { -- }
$$

$11.8\left(10^{6}\right)$

$--$

Air Source Heat Pump C
$13.3\left(10^{6}\right)$
$57.7\left(10^{6}\right)$
$11.9 \%$
$13.5\left(10^{6}\right)$
$11 \%$
$25.7\left(10^{6}\right)$
$13.2 \%$

$22.4\left(10^{6}\right)$

$.4 \%$

*Energy represents: energy input at power plant for electrical power. 
Table 10: Energy* Comparison for Heating and Cooling Well Insulated Typical Home Percentage Savings Utilizing Proposed Modified Water Source Heat Pump A With Pre-Water Heat Exchanger -- Alternative I

$\begin{array}{llll}\text { BIRMINGHAM NEW YORK MIAMI } \quad \text { DETROIT } & \text { ST. LOUIS }\end{array}$

Total Energy Input Btu

$\begin{array}{ccc}\text { Proposed Water Source Heat Pump System } \\ 5.93\left(10^{7}\right) & 5.78\left(10^{7}\right) & 4.4\left(10^{7}\right)\end{array}$

$7.29\left(10^{7}\right)$

$6.61\left(10^{7}\right)$

Resistance Electric Heating and Air Source Heat Pump C Cooling Total Energy Input Btu
Relative Savings

Total Energy Input Btu Relative Savings

Total Energy Input Btu Relative Savings

Total Energy Input Btu Relative Savings

$$
14.7\left(10^{7}\right)
$$$$
59.7 \%
$$

$$
6.3\left(10^{7}\right)
$$$$
30.2 \%
$$

$2.04\left(10^{8}\right)$

$64.3 \%$

Gas and Oil Heating and Air Source Heat Pump C Cooling
$9.95\left(10^{7}\right)$
$40.4 \%$
$9.48\left(10^{7}\right)$
$39 \%$
$6.07\left(10^{7}\right)$
$1.2\left(10^{8}\right)$
$39.3 \%$
$1.07\left(10^{8}\right)$
$38.2 \%$

Air Source Heat Pump A

$7.26\left(10^{7}\right)$
$18.3 \%$

$7.36\left(10^{7}\right)$

$21.5 \%$

$5.24\left(10^{7}\right)$

$9.98\left(10^{7}\right)$

$27 \%$

$8.76\left(10^{7}\right)$

$24.5 \%$

Air Source Heat Pump C

$9.24\left(10^{7}\right)$
$35.8 \%$

$8.88\left(10^{7}\right)$

$34.9 \%$ $6.0\left(10^{7}\right)$

$26.7 \%$ $1.22\left(10^{8}\right)$

$40.2 \%$ $1.05\left(10^{8}\right)$

$37 \%$

*Energy represents energy input at power plant for electrical power plus higher heating value of fuel for furnace input. 
Table 11: Energy* Comparison for Heating Well Insulated Typical Home Percentage Savings Utilizing Proposed Modified Water Source Heat Pump A With Pre-Water Heat Exchanger -- Alternative I

BIRMINGHAM NEW YORK MIAMI DETROIT ST. LOUIS

Total Energy Input Btu

Total Energy Input Btu Relative Savings

$\therefore$

Total Energy Input Btu Relative Savings

Total Energy Input Btu Relative Savings

Total Energy Input Btu Relative Savings
Proposed Water Source Heat Pump System

$$
36.5\left(10^{6}\right)
$$

$$
47.8\left(10^{6}\right)
$$

$1.77\left(10^{6}\right)$

$62.9\left(10^{6}\right)$

$47.5\left(10^{6}\right)$

$$
110\left(10^{6}\right)
$$

$66.8 \%$

$62.2\left(10^{6}\right)$
$41.3 \%$

$45.3\left(10^{6}\right)$
$19.4 \%$

$55.1\left(10^{6}\right)$

$33.8 \%$
Electric Resistance Heating

$\begin{array}{llll}144\left(10^{6}\right) & 53.4\left(10^{5}\right) & 190\left(10^{6}\right) & 143\left(10^{6}\right) \\ 66.8 \% & 66.8 \% & 66.9 \% & 66.8 \%\end{array}$

Gas and $0 i 1$ Heating

$\begin{array}{llll}81.5\left(10^{6}\right) & 30.2\left(10^{5}\right) & 107\left(10^{6}\right) & 81\left(10^{6}\right) \\ 41.3 \% & 41.3 \% & 41.2 \% & 41.3 \%\end{array}$

Air Source Heat Pump A

$\begin{array}{llll}61.9\left(10^{6}\right) & 1.98\left(10^{6}\right) & 88\left(10^{6}\right) & 55.2\left(10^{6}\right) \\ 22.8 \% & 10.6 \% & 28.5 \% & 27.1 \%\end{array}$

Air Source Heat Pump C

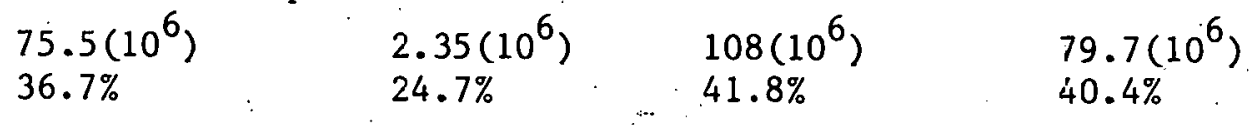

*Energy represents energy input at power plant for electrical power plus higher heating value of fuel for furnace input. 
Table 12: Energy* Comparison for Cooling Well Insulated Typical Home Percentage Savings Utilizing Proposed Modified Water Source Heat Pump A With Pre-Water Heat Exchanger -- Alternative I

$\begin{array}{lllll}\text { BIRMINGHAM NEW YORK } & \text { MIAMI } & \text { DETROIT } & \text { ST LOUIS }\end{array}$

Total Energy Input Btu

Total Energy Input Btu Relative Savings

Total Energy Input Btu Relative Savings

Proposed Water Source Heat Pump System
$22.8\left(10^{6}\right)$
$10.0\left(10^{6}\right)$
$42.4\left(10^{6}\right)$
$10.0\left(10^{6}\right)$
$18.6\left(10^{6}\right)$

Air Source Heat Pump A

$$
\begin{aligned}
& 27.3\left(10^{6}\right) \\
& 16.5 \%
\end{aligned}
$$

$$
\begin{aligned}
& 11.7\left(10^{6}\right) \\
& 14.5 \%
\end{aligned}
$$

$50.4\left(10^{6}\right)$

$15.9 \%$

$11.8\left(10^{6}\right)$
$15.3 \%$

Air Source Heat Pump C

$37.3\left(10^{6}\right)$
$38.9 \%$

$13.3\left(10^{6}\right)$

$24.8 \%$ $57.7\left(10^{6}\right)$

$26.5 \%$ $13.5\left(10^{6}\right)$

$25.9 \%$ $22.4\left(10^{6}\right)$ $17 \%$

$25.7\left(10^{6}\right)$ $27.6 \%$ 
Table 13: Energy* Comparison for Heating and Cooling Well Insulated Typical Home

Percentage Savings Utilizing Proposed Modified Water Source Heat Pump A With Pre-Water Heat Exchanger - Alternative II

BIRMINGHAM NEW YORK $\because \cdots$ MIAMI DETROIT $\quad$ ST. LOUIS

Total Energy Input Btu

Proposed Water Source Heat Pump System

$$
4.75\left(10^{7}\right)
$$
Resistance Electric Heating and Air Source Heat Pump C Cooling
Total Energy Input Btu
$1.47\left(10^{8}\right)$
$1.5\left(10^{8}\right)$
$6.3\left(10^{7}\right)$
$2.04\left(10^{8}\right)$
$1.69\left(10^{8}\right)$
$64.8 \%$
Relative Savings
$67.7 \%$

$$
61.4 \% \text {. }
$$
$81.7 \%$
$62.5 \%$$$
5.79\left(10^{7}\right)
$$$$
1.15\left(10^{7}\right) \text {. }
$$$$
7.56\left(10^{7}\right)
$$$$
5.95\left(10^{7}\right)
$$

Gas and Oil Heating and Air Source Heat Pump C Cooling

Total Energy Input Bțu Relative Savings

Total Energy Input Btu Relative Savings

Total Energy Input Btu Relative Savings

$$
\begin{aligned}
& 9.95\left(10^{7}\right) \\
& 52.3 \%
\end{aligned}
$$

$$
\begin{aligned}
& 9.48\left(10^{7}\right) \\
& 38.9 \%
\end{aligned}
$$

$6.07\left(10^{7}\right)$

$81 \%$

$1.2\left(10^{8}\right)$
$37 \%$

$1.07\left(10^{8}\right)$

$44.4 \%$

Air Source Heat. Pump A

$$
\begin{aligned}
& 7.26\left(10^{7}\right) \\
& 34.6 \%
\end{aligned}
$$

Air Source Heat Pump C

$$
\begin{aligned}
& 9.24\left(10^{7}\right) \\
& 48.6 \%
\end{aligned}
$$

$$
7.36\left(10^{7}\right)
$$

$$
21.3 \%
$$

$$
5.24\left(10^{7}\right)
$$$$
78.1 \%
$$

$6.0\left(10^{7}\right)$ $80.8 \%$

$$
9.98\left(10^{7}\right)
$$$$
24.2 \%
$$$$
8.76\left(10^{7}\right)
$$$$
32.1 \%
$$

$$
8.88\left(10^{7}\right)
$$$$
34.8 \%
$$

$1.22\left(10^{8}\right)$
$38 \%$ $1.05\left(10^{8}\right)$

*Energy represents energy input at power plant for electrical power plus higher heating value of fuel for. furnace input. 
Table 14: Energy* Comparison for Heating Well Insulated Typical Home Percentage Savings Utilizing Proposed Modified Water Source Heat Pump A. With Pre-Water Heat Exchanger -- Alternative II

BIRMINGHAM NEW YORK MIAMI : DETROIT $\quad$ ST. LOUIS

Total Energy Input Btu

Total Energy Input Btu Relative Savings

Total Energy Input Btu Relative Savings

Total Energy Input Btu Relative Savings

Total Energy Input Btu Relative Savings

Proposed Water Source Heat Pump System
$42.5\left(10^{6}\right)$
$55.7\left(10^{6}\right)$
$2.06\left(10^{6}\right)$
$73.4\left(10^{6}\right)$
$55.4\left(10^{6}\right)$

Electric Resistance Heating

$\begin{array}{lllll}110\left(10^{6}\right) & 144\left(10^{6}\right) & 5.34\left(10^{6}\right) & 190\left(10^{6}\right) & 143\left(10^{6}\right) \\ 61.4 \% & 61.4 \% & 61.4 \% & 61.4 \% & 61.3 \%\end{array}$

Gas and $0 i 1$ Heating

$\begin{array}{lllll}62.2\left(10^{6}\right) & 81.5\left(10^{6}\right) & 3.0\left(10^{6}\right) & 107\left(10^{6}\right) & 81.0\left(10^{6}\right) \\ 31.7 \% & 31.7 \% & 31.4 \% & 31.4 \% & 31.6 \%\end{array}$

Air Source Heat Pump. A

$45.3\left(10^{6}\right)$
$6.2 \%$

$$
\begin{aligned}
& 61.9\left(10^{6}\right) \\
& 10 \%
\end{aligned}
$$

$$
1.98\left(10^{6}\right)
$$

Air Source Heat Pump C

$$
\begin{aligned}
& 55.1\left(10^{6}\right) \\
& 22.9 \%
\end{aligned}
$$
$2.35\left(10^{6}\right)$ $12.3 \%$

$31.6 \%$

$65.2\left(10^{6}\right)$ $15 \%$

$79.7\left(10^{6}\right)$ $30.5 \%$

*Energy represents energy input at power plant for electrical power plus higher heating value of fuel for furnace input. 
Table 15: Energy* Comparison for. Cooling Well Insulated Typical Home Percentage Savings Utilizing Proposed Modified Water Source Heat Pump A With Pre-Water Heat Exchanger -- Alternative II

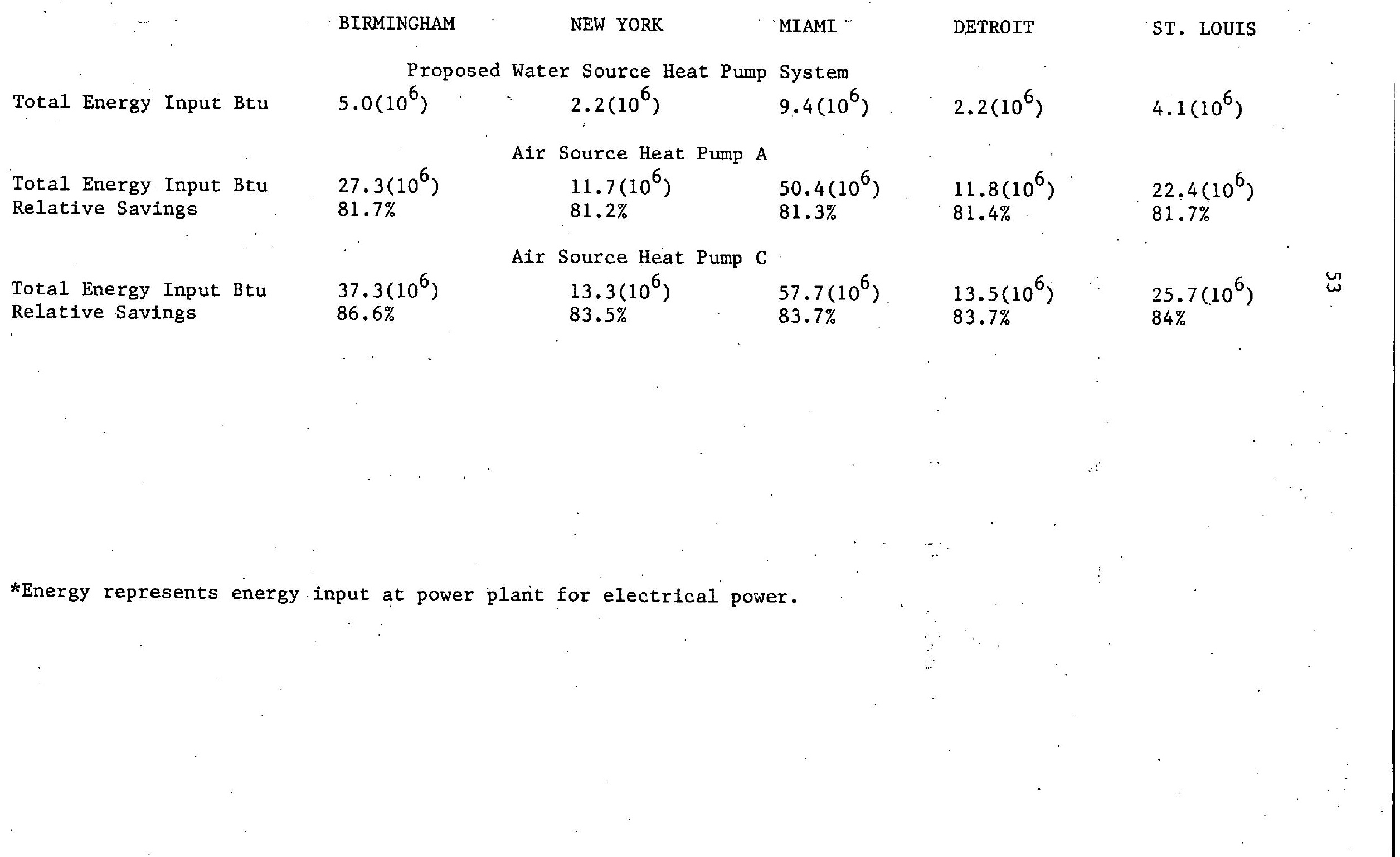


Table 16: Energy* Comparison for Heating and Cooling Well Insulated Typical Home Percentage Savings Utilizing Proposed Modified Water Source Heat Pump A With Pre-Water Heat Exchanger -- Alternative III
BIRMINGHAM
NEW YORK
MIAMI
DETROIT
ST. LOUIS

Total Energy Input Btu

$4.66\left(10^{7}\right)$

Proposed Water Source Heat Pump System

\section{Total Energy Input Btu} Relative Savings

\section{Resis}

esistance Electr$$
3.29\left(10^{7}\right)
$$

$6.07\left(10^{7}\right)$

$3.88\left(10^{7}\right)$

$4.5\left(10^{7}\right)$

\section{Total Energy Input Btu Relative Savings.}

Total Energy Input Btu Relative Savings

Total Energy Input Btu Relative Savings

$$
1.47\left(10^{8}\right)
$$$$
68.3 \%
$$

Gas and $0 i 1$ Heating and Air Source Heat Pump C Cooling
$9.95\left(10^{7}\right)$
$53.2 \%$
$9.48\left(10^{7}\right)$
$65.3 \%$
$1.5\left(10^{8}\right)$
$1.5\left(10^{8}\right)$
$78 \%$

$6.3\left(10^{7}\right)$

$3.6 \%$

$6.07\left(10^{7}\right)$

$1.2\left(10^{8}\right)$

$1.2(10$
$67.7 \%$

$2.04\left(10^{8}\right)$

$81 \%$

$1.69\left(10^{8}\right)$ $73.4 \%$

Air Source Heat Pump A

$7.26\left(10^{7}\right)$
$35.8 \%$

$$
7.36\left(10^{7}\right)
$$

$55.3 \%$
$5.24\left(10^{7}\right)$
$-15.8 \%$
$9.98\left(10^{7}\right)$
$61.1 \%$

$$
1.07\left(10^{8}\right)
$$$$
57.9 \%
$$

Air Source Heat Pump C

$$
\begin{aligned}
& 9.27\left(10^{7}\right) \\
& 49.7 \%
\end{aligned}
$$

$$
8.88\left(10^{7}\right)
$$$$
62.9 \%
$$

$$
6.0\left(10^{7}\right)
$$$$
-1.2 \%
$$

$$
\begin{aligned}
& 1.22\left(10^{8}\right) \\
& 68.2 \%
\end{aligned}
$$

\section{$8.76\left(10^{7}\right)$ $48.6 \%$}

$$
\begin{aligned}
& 1.05\left(10^{8}\right) \\
& 57.1 \%
\end{aligned}
$$

*Energy represents energy input at power plant for electrical power plus higher heating value of fuel for furnace input. 
Table 17: Energy* Comparison for Heating Well Insulated Typical Home Percentage Savings Utilizing Proposed Modified Water Source Heat Pump A With Pre-Water Heat Exchanger -- Alternative III

$\begin{array}{llll}\text { BIRMINGHAM NEW YORK MIAMI DETROIT } & \text { ST. LOUIS }\end{array}$

Total Energy Input Btu

Total Energy Input Btu Relative Savings

Total Energy Input Btu Relative Savings

Total Energy Input Btu Relative Savings

Total Energy Input Btu Relative Savings

Proposed Water Source Heat Pump System

$$
\begin{array}{lllll}
14.2\left(10^{6}\right) & 18.6\left(10^{6}\right) & 6.9\left(10^{5}\right) & 24.5\left(10^{6}\right) & 18.5\left(10^{6}\right)
\end{array}
$$

$$
\begin{aligned}
& 110\left(10^{6}\right) \\
& 87 \%
\end{aligned}
$$

\begin{tabular}{|c|c|c|}
\hline $\begin{array}{l}144\left(10^{6}\right) \\
87 \%\end{array}$ & $\begin{array}{l}53.4\left(10^{5}\right) \\
87 \%\end{array}$ & $\begin{array}{l}190\left(10^{6}\right) \\
87 \%\end{array}$ \\
\hline
\end{tabular}

$$
62.2\left(10^{6}\right)
$$$$
77.2 \%
$$

$45.3\left(10^{6}\right)$

$68.7 \%$

$$
\begin{aligned}
& 55.1\left(10^{6}\right) \\
& 74.2 \%
\end{aligned}
$$

Electric Resistance Heating

Gas and 0il Heating

$\begin{array}{llll}81.5\left(10^{6}\right) & 30.2\left(10^{5}\right) & 107\left(10^{6}\right) & 81\left(10^{6}\right) \\ 77.2 \% & 77.2 \% & 77.1 \% & 77.2 \%\end{array}$

Air Source Heat Pump A

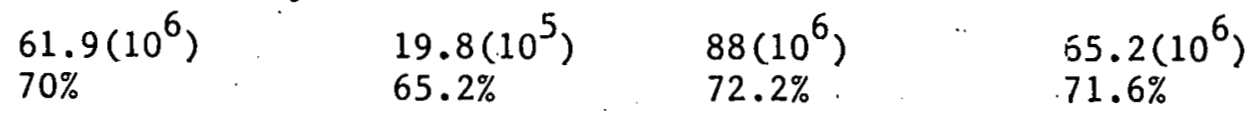

Air Source Heat Pump C

$$
\begin{array}{llll}
75.5\left(10^{6}\right) & 23.5\left(10^{5}\right) & \ldots 108\left(10^{6}\right) & 79.7\left(10^{6}\right) \\
75.4 \% & 70.6 \% & 77.3 \% & 76.8 \%
\end{array}
$$

*Energy represents energy input at power plant for electrical power plus higher heating value of fuel for furnace input. 
Table 18: Energy* Comparison for Cooling Well Insulated Typical Home Percentage Savings Utilizing Proposed Modified Water Source Heat Pump A With Pre-Water Heat Exchanger -- Alternative III

BIRMINGHAM

NEW YORK

MIAMI

DETROIT

ST. LOUIS

Proposed Water Source Heat Pump System

Total Energy Input Btu

Total Energy Input Btu Relative Savings

Total Energy Input Btu Relative Savings

$$
14.3\left(10^{6}\right)
$$$$
60\left(10^{6}\right)
$$

$14.3\left(10^{6}\right)$

$26.5\left(10^{6}\right)$

Air Source Heat Pump. A $27.3\left(10^{6}\right)$

$-18.7 \%$

$$
11.7\left(10^{6}\right)
$$$$
-22.2 \%
$$

$50.4\left(10^{6}\right)$

$-19 \%$

$11.8\left(10^{6}\right)$

$-21.2 \%$

$22.4\left(10^{6}\right)$ $-18.3 \%$

Air Source Heat Pump C

$37.3\left(10^{6}\right)$
$13.1 \%$

$$
13.3\left(10^{6}\right)
$$$$
-7.5 \%
$$

$13.5\left(10^{6}\right)$

$-5.9 \%$ $25.7\left(10^{6}\right)$ $-3.1 \%$

*Energy represents energy input at power plant for electrical power. 


\section{EXPECTED ECONOMICS}

In general, economics for the heat pump centered integrated community energy system are expected to be superior to present systems. Comparisons are made with gas, oll, and electric systems. For comparisons with electric systems (air source heat pumps or resistance heating plus air conditioning) both operating costs and capital costs are favorable. In comparison with oil or gas heating and air conditioning, operating costs vary with fuel costs as a function of location. All comparisons include air conditioning as the proposed system utilizes air conditioning. Some parts of the country, especially with respect to residential buildings, have not experienced $30 \%$ saturation yet on air conditioning. 2 No comparisons have been made with heating alone.

The economic effect of lower capacity requirements and load leveling on power plants has not been included. Alternative II (direct cooling) in particular can cut summer peak loads by over $30 \%$ (reducing air conditioning loads by $60 \%$ ) in belts across the Midsouth. As a result capital outlays are reduced and plant productivity and efficiency increase. These are major favorable economic factors for the proposed system and alternatives.

The proposed system has different capital costs for the heating and cooling system in the home as compared with small business and commercial installations. In the home or small commercial establishment a furnace and air conditioner or an air source heat pump is replaced by a water source heat pump. The basic unit is a complete package with an integrated tube-in-tube heat exchanger. This system is expected to be ownerowned. In commercial systems designed by owner and the engineering con- 
sultant, the present units, mainly chillers, are already water-source and can be converted to the proposed water supplies. In addition, a redesign of the system can include heating. The need for a furnace is eliminated. The requirement for cooling towers is eliminated, but they could be used to reject excess heat in southern parts of the country or in areas where buildings have large internal loads. Again, the internal system is privately owned. Based on these variations, it is assumed that, for economic analysis, capital costs are proportional to cost of the 3-ton units used in small buildings. Some errors will arise, especially in larger buildings where numerous combinations of internal loads and HVAC systems exist. A 3-ton water source unit operating in this system provides well over 4 tons of heating and 3 tons of cooling. It will meet the load capacities for the well-insulated building for both heating and cooling in over $90 \%$ of the country. In addition, the installed price for furnace plus central air conditioner is assumed to be the same as the installed price for air source heat pump $C$.

Capital costs of installed units for heating and cooling are therefore taken from Christian. $10,1 \mathrm{l}$ should be noted the installed price of a water source heat pump is basically one-half that of an installed air source heat pump. This results from the use of 1 packaged unit rather than 2 packaged units. The basic water source heat pump is also simpler and smaller than the air source heat pump. A surcharge of $\$ 300$ has been added to the air source heat pump A for exceptionally high quality. Few of these units are in service. The capital cost for the installed heating and cooling equipment used for the economic analysis is: 
Furnace and Air-Conditioner

$\$ 2500$

Air Source Heat Pump A

2800

Air Source Heat Pump C

2500

Water Source Heat Pump A

1300

Modified Water Source Heat Pump A (a11 3 alternatives) 1600

In addition, for the proposed system wells, pumping systems, energy addition, and a water distribution system are required. The installed capital costs for each of these components are included in the analysis section IX. The capital costs per module are:

$$
\begin{aligned}
& \text { Well Construction ( } 2 \text { wells) } \\
& \text { Pumping. System (pump, motor and controls)(2 systems) } 6000 \\
& \text { Capacitance Tank ( } 2 \text { tanks) } \\
& \text { Solar Collector (1000 } \left.\mathrm{ft}^{2} \text { at } \$ 3 / \mathrm{ft}^{2}\right) \text {. } \\
& \text { Water Distribution System (primary and secondary) } \\
& 1000 \mathrm{ft} \text {. of Double 6-inch Main } \\
& \text { 1000. ft. of Double } 1 \frac{1}{2}-\text { inch Secondary } 3400 \\
& \begin{array}{ll}
\text { Trench }(2000 \mathrm{ft} . \text { at } \$ 1.47 / \mathrm{ft} .) & 2940 \\
\text { Total Water System Capital Cost } & \frac{15540}{\$ 27800}
\end{array} \\
& \text { Total Cost per Home in a } 20 \text {-Home Module } \$ 1390
\end{aligned}
$$

Annual capital cost is based on an effective $10 \%$ interest rate plus 12

a sinking-fund depreciation over 20 years at the same $10 \%$ annual rate.

It must be noted interest rates vary and that public utilities and pri-. vate utilities have access to different rates of interest. All equipment except solar collectors is expected to have lifetimes in excess of 20 years. The relatively small cost of energy addition makes the 20-year depreciation rate reasonable. Utilizing this criterion, the annual capital cost is $10 \%$ interest plus $1.7 \%$ depreciation for a total of $11.7 \%$ of the total capital cost. The annual capital cost for the system per home is:

\begin{tabular}{lcc} 
& Total Capital & Annual Cost \\
\cline { 2 - 3 } Furnace and Air Conditioner & $\$ 2500$ & $\$ 292.50$ \\
Air Source Heat Pump A & 2800 & 327.60 \\
Air Source Heat Pump C & 2500 & 292.50 \\
Water Source Heat Pump A & 2690 & $: 314.73$ \\
in Proposed System & & \\
Modified Water Source Heat Pump & 2990 & 349.83 \\
A Plus System (Includes all & & $\therefore$ \\
3 alternatives) & & $\therefore$
\end{tabular}


Annual maintenance costs based on Chri'stian are $\$ 165$ for both the air source and water source heat pumps for the first 5 years. 10,11 After. this period, maintenance costs are expected to increase at the rate of $7 \%$ per year. Due to simplicity and a low pressure ratio, the water source heat pumps should be expected to have a lower maintenance cost. Based on this assumption, maintenance costs for both the air source heat pumps and the proposed system are assumed to be an average of $25 \%$ higher than the $\$ 165$ over the 20 years. An average annual maintenance cost of $\$ 205$ is used in the economic analysis. Assuming water source heat pump maintenance is $25 \%$ less than that of an air source unit allows the utility $\$ 1020$ per year maintenance for 20 homes for the water distribution sysstem and wells for one module. Resistance heating and furnace plus the air conditioner is given an annual maintenance cost of $\$ 150$ as compared with heat pump maintenance. The energy required for water pumping is included in the water source heat pump energy requirements.

Tables 19 to 21 show the annual energy cost and annual total cost

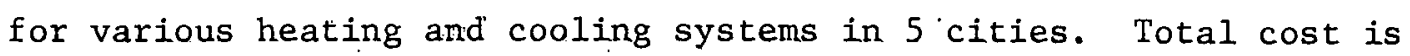
energy cost, capital cost, and maintenance cost combined. Electricity costs vary from $\$ .04$ to $\$ 0.12$ per $\mathrm{Kw} / \mathrm{hr}$ and fuel cost is set at $\$ 2.50$ per $10^{6}$ Btu.

A gas and oil cost of $\$ 3.50$ to $\$ 4.50$ per $10^{6} \mathrm{Btu}$ is reasonable in some parts of the country which represents a much higher cost for gas and oil heating energy, in the comparisons. In turn the energy cost for gas and oil heating is much higher than the tables indicate.

In general, the costs for the proposed systems and alternatives are favorable on both a fuel and a capital cost basis. 
Table 19: Economic Analysis for Annual Heating and Cooling for Well Insulated Typical Home (\$0.04 Per $\mathrm{Kw}-\mathrm{hr}$ Electric Cost $)$

BIRMINGILAM NEW YORK MIAMI DETROIT ST. LOUIS

Resistance Electric Heating and Air Source Heat Pump C Cooling

$\begin{array}{llllll}\text { Power Input } \mathrm{Kw}-\mathrm{hr} & 18.6\left(10^{3}\right) & 16.2\left(10^{3}\right) & 6.5\left(10^{3}\right) & 20.6\left(10^{3}\right) & 17.3\left(10^{3}\right) \\ \text { Annual Electric Cost } & \$ 744 & \$ 648 & \$ 260 & \$ 836 & \$ 692 \\ \text { Total Annual Cost } & \$ 1187 & \$ 1091 & \$ 703 & \$ 1279 & \$ 1135\end{array}$

Gas and Oil Heating and Air Source Heat Pump C Cooling ( $\$ 2.50$ Per $10^{6}$ Btu Fuel Cost)

\begin{tabular}{|c|c|c|c|c|c|}
\hline Combustion Energy & $62.2\left(10^{6}\right)$ & $81.5\left(10^{6}\right)$ & $30.2\left(10^{5}\right)$ & $107\left(10^{6}\right)$ & $81\left(10^{6}\right)$ \\
\hline Combustion Energy Cost & $\$ 156$ & $\$ 204$ & $\$ 76$ & $\$ 268$ & $\$ 203$ \\
\hline Power Input $\mathrm{Kw}-\mathrm{hr}$ & $3.83\left(10^{3}\right)$ & $1.37\left(10^{3}\right)$ & $5.92\left(10^{3}\right)$ & $1.38\left(10^{3}\right)$ & $2.64\left(10^{3}\right)$ \\
\hline Electric Cost & $\$ 153$ & $\$ 55$ & $\$ 237$ & $\$ 55$ & $\$ 106$ \\
\hline Annual Energy Cost & $\$ 309$ & $\$ 259$ & $\$ 313$ & $\$ 323$ & $\$ 309$ \\
\hline Total Annual Cost & $\$ 752$ & $\$ 702$ & $\$ 756$ & $\$ 766$ & $\$ 752$ \\
\hline \multicolumn{6}{|c|}{ Air Source Heat Pump A } \\
\hline Power Input $\mathrm{Kw}-\mathrm{hr}$ & $7.5\left(10^{3}\right)$ & $7.54\left(10^{3}\right)$ & $5.4\left(10^{3}\right)$ & $10.2\left(10^{3}\right)$ & $9.3\left(10^{3}\right)$ \\
\hline Annual Electric Cost & $\$ 300$ & $\$ 302$ & $\$ 216$ & $\$ 408$ & $\$ 373$ \\
\hline Total Annual Cost & $\$ 833$ & $\$ 835$ & $\$ 749$. & $\$ 941$ & $\$ 906$ \\
\hline \multicolumn{6}{|c|}{ Air Source Heat Pump C } \\
\hline Power Input $\mathrm{Kw}_{\mathrm{w}} \mathrm{hr}$ & $9.5\left(10^{3}\right)$ & $9.1\left(10^{3}\right)$ & $6.2\left(10^{3}\right)$ & $12.5\left(10^{3}\right)$ & $10.8\left(10^{3}\right)$ \\
\hline Annual Electric Cost & $\$ 380$ & $\$ 363$ & $\$ 248$ & $\$ 500$ & $\$ 432$ \\
\hline Total Annual Cost & $\$ 878$ & $\$ 861$ & $\$ 746$ & $\$ 998$ & $\$ 930$ \\
\hline \multicolumn{6}{|c|}{ Water Source Heat Pump A } \\
\hline ower In & $6.9\left(10^{3}\right)$ & $6.6\left(10^{3}\right)$ & $5.2\left(10^{3}\right)$ & $8.3\left(10^{3}\right)$ & $7.6\left(10^{3}\right)$ \\
\hline nnual Electric Cost & $\$ 276$ & $\$ 264$ & $\$ 208$ & $\$ 332$ & $\$ 304$ \\
\hline Total Annual Cost & $\$ 796$ & $\$ 784$ & $\$ 728$ & $\$ 852$ & $\$ 824$ \\
\hline
\end{tabular}

Modified Water Source. Heat Pump A With Pre-Water Heat Exchanger -- Alternative I

$\begin{array}{llllll}\text { Power Input } \mathrm{Kw}-\mathrm{hr} & 6.1\left(10^{3}\right) & 5.9\left(10^{3}\right) & 4.5\left(10^{3}\right) & 7.5\left(10^{3}\right) & 6.8\left(10^{3}\right) \\ \text { Annual Electric Cost } & \$ 244 & \$ 236 & \$ 180 & \$ 300 & \$ 272 \\ \text { Total Annual Cost } & \$ 799 & \$ 791 & \$ 735 & \$ 855 & \$ 827\end{array}$

Modified Water Source Heat Pump A With Pre-Water Heat Exchanger -- Alternative II

$\begin{array}{llllll}\text { Power Input Kw-hr } & 4.9\left(10^{3}\right) & 5.9\left(10^{3}\right) & 1.2\left(10^{3}\right) & 7.8\left(10^{3}\right) & 6.1\left(10^{3}\right) \\ \text { Annual Electric Cost } & \$ 196 & \$ 236 & \$ 48 & \$ 312 & \$ 244 \\ \text { Total Annual Cost } & \$ 751 & \$ 791 & \$ 603 & \$ 867 & \$ 799\end{array}$

Modified Water Source Heat Pump A With Pre-Water Heat Exchanger -- Alternative III

$\begin{array}{llllll}\text { Power Input } \mathrm{Kw}-\mathrm{hr} & 4.8\left(10^{3}\right) & 3.4\left(10^{3}\right) & 6.2\left(10^{3}\right) & 3.9\left(10^{3}\right) & 4.6\left(10^{3}\right) \\ \text { Annual Electric Cost } & \$ 192 & \$ 136 & \$ 248 & \$ 156 & \$ 184 \\ \text { Total Annual Cost } & \$ 747 & \$ 691 & \$ 803 & \$ 911 & \$ 739\end{array}$


Table 20: Economic Analysis for Annual Heating and

Cooling for Well Insulated Typical Home.

( $\$ 0.08$ Per $\mathrm{Kw}-\mathrm{hr}$ Electric Cost)

\section{BIRMINGHAM NEW YORK MIAMI DETROIT ST. LOUIS}

Resistance Electric Heating and Air Source Heat Pump C Cooling

\begin{tabular}{|c|c|c|c|c|c|}
\hline $\begin{array}{l}\text { Power Input } \mathrm{Kw}-\mathrm{hr} \\
\text { Annual Electric Cost } \\
\text { Total Annual Cost }\end{array}$ & $\begin{array}{l}18.6\left(10^{3}\right) \\
\$ 1488 \\
\$ 1931\end{array}$ & $\begin{array}{l}16.2\left(10^{3}\right) \\
\$ 1296 \\
\$ 1739\end{array}$ & $\begin{array}{l}6.5\left(10^{3}\right) \\
\$ 520 \\
\$ 963\end{array}$ & $\begin{array}{l}20.9\left(10^{3}\right) \\
\$ 1672 \\
\$ 2115\end{array}$ & $\begin{array}{l}17.3\left(10^{3}\right) \\
\$ 1384 \\
\$ 1827\end{array}$ \\
\hline Gas and & \multicolumn{5}{|c|}{$\begin{array}{l}\text { Oil Heating and Ajr Source Heat Pump C Cooling } \\
\left(\$ 2.50 \text { Per } 10^{6} \text { Btu Fuel Cost }\right)\end{array}$} \\
\hline Combustion Energy & $62.2\left(10^{6}\right)$ & $81.5\left(10^{6}\right)$ & $30.2\left(10^{5}\right)$ & $107\left(10^{6}\right)$ & $81\left(10^{6}\right)$ \\
\hline Combustion Energy Cost & $\$ 156$ & $\$ 204$ & & $\$ 268$ & $\$ 203$ \\
\hline Power Input $\mathrm{Kw}-\mathrm{hr}$ & $3.83\left(10^{3}\right)$ & $1.37\left(10^{3}\right)$ & $5.92\left(10^{3}\right)$ & $1.38\left(10^{3}\right)$ & $2.64\left(10^{3}\right)$ \\
\hline Electric Cost & $\$ 306$ & $\$ 110$ & $\$ 474$ & $\$ 110$ & $\$ 211$ \\
\hline Annual Energy Cost & $\$ 462$ & $\$ 314$ & $\$ 550$ & $\$ 378$ & $\$ 414$ \\
\hline Total Annual Cost & $\$ 905$ & $\$ 757$ & $\$ 993$ & $\$ 821$ & $\$ 857$ \\
\hline \multicolumn{6}{|c|}{ Air Source Heat Pump A } \\
\hline Power Input $\mathrm{Kw}-\mathrm{hr}$ & $7.5\left(10^{3}\right)$ & $7.54\left(10^{3}\right)$ & $5.4\left(10^{3}\right)$ & $10.2\left(10^{3}\right)$ & $9 \cdot 3\left(10^{3}\right)$ \\
\hline Annual Electric Cost & $\$ 600$ & $\$ 603$ & $\$ 432$ & $\$ 816$ & $\$ 744$ \\
\hline Total Annual Cost & $\$ 1133$ & $\$ 1136$ & $\$ 965$ & $\$ 1349$ & $\$ 1277$ \\
\hline \multicolumn{6}{|c|}{ Air Source Heat Pump C } \\
\hline Power Input $\mathrm{Kw}-\mathrm{hr}$ & $9.5\left(10^{3}\right)$ & $9.1\left(10^{3}\right)$ & $6.2\left(10^{3}\right)$ & $12.5\left(10^{3}\right)$ & $10.8\left(10^{3}\right)$ \\
\hline Annual Electric Cost & $\$ 760$ & $\$ 728$ & $\$ 496$ & $\$ 1000$ & $\$ 864$ \\
\hline Total Annual Cost & $\$ 1258$ & $\$ 1226$ & $\$ 994$ & $\$ 1498$ & $\$ 1362$ \\
\hline \multicolumn{6}{|c|}{ Water Source Heat Pump A } \\
\hline $\begin{array}{l}\text { Power Input } \mathrm{Kw}-\mathrm{hr} \\
\text { Annual Electric Cost }\end{array}$ & $\begin{array}{l}6.9\left(10^{3}\right) \\
\$ 552\end{array}$ & $\begin{array}{l}6.6\left(10^{3}\right) \\
\$ 528\end{array}$ & $\begin{array}{l}5.2\left(10^{3}\right) \\
\$ 416\end{array}$ & $\begin{array}{l}8.3\left(10^{3}\right) \\
\$ 664\end{array}$ & $\begin{array}{l}7.6\left(10^{3}\right) \\
\$ 608\end{array}$ \\
\hline Total Annual Cost & $\$ 1072$ & $\$ 1048$ & $\$ 936$ & $\$ 1184$ & $\$ 1128$ \\
\hline
\end{tabular}

Modified Water Source Heat Pump A With Pre-Water Heat Exchanger -- Alternative I

$\begin{array}{llllll}\text { Power Input Kw-hr } & 6.1\left(10^{3}\right) & 5.9\left(10^{3}\right) & 4.5\left(10^{3}\right) & 7.5\left(10^{3}\right) & 6.8\left(10^{3}\right) \\ \text { Annual Electric Cost } & \$ 488 & \$ 472 & \$ 360 & \$ 600 & \$ 544 \\ \text { Total Annual Cost } & \$ 1043 & \$ 1027 & \$ 915 & \$ 1155 & \$ 1099\end{array}$

Modified Water Source Heat Pump A With Pre-Water Heat Exchanger -- Alternative II

$\begin{array}{llllll}\text { Power Input Kw-hr } & 4.9\left(10^{3}\right) & 5.9\left(10^{3}\right) & 1.2\left(10^{3}\right) & 7.8\left(10^{3}\right) & 6.1\left(10^{3}\right) \\ \text { Annual Electric Cost } & \$ 392 & \$ 472 & \$ 96 & \$ 624 & \$ 488 \\ \text { Total Annual Cost } & \$ 947 & \$ 1027 & \$ 651 & \$ 1179 & \$ 1043\end{array}$

Modified Water Source Heat Pump A With Pre-Water. Heat Exchanger -- Alternative III

$\begin{array}{llllll}\text { Power Input } \mathrm{Kw}-\mathrm{hr} & 4.8\left(10^{3}\right) & 3.4\left(10^{3}\right) & 6.2\left(10^{3}\right) & 3.9\left(10^{3}\right) & 4.6\left(10^{3}\right) \\ \text { Annual Electric Cost } & \$ 384 & \$ 272 & \$ 496 & \$ 312 & \$ 368 \\ \text { Total Annual Cost } & \$ 939 & \$ 827 & \$ 1051 & \$ 867 & \$ 923\end{array}$


Table 21: Economic Analysis for Annual Heating and Cooling for Well Insulated Typical Home (\$0.12 Per $\mathrm{Kw}-\mathrm{hr}$ Electric Cost)

- BIRMINGHAM NEW YORK MIAMI DETROIT . ST. LOUIS

Resistance Electric Heating and Air Source Heat Pump C Cooling

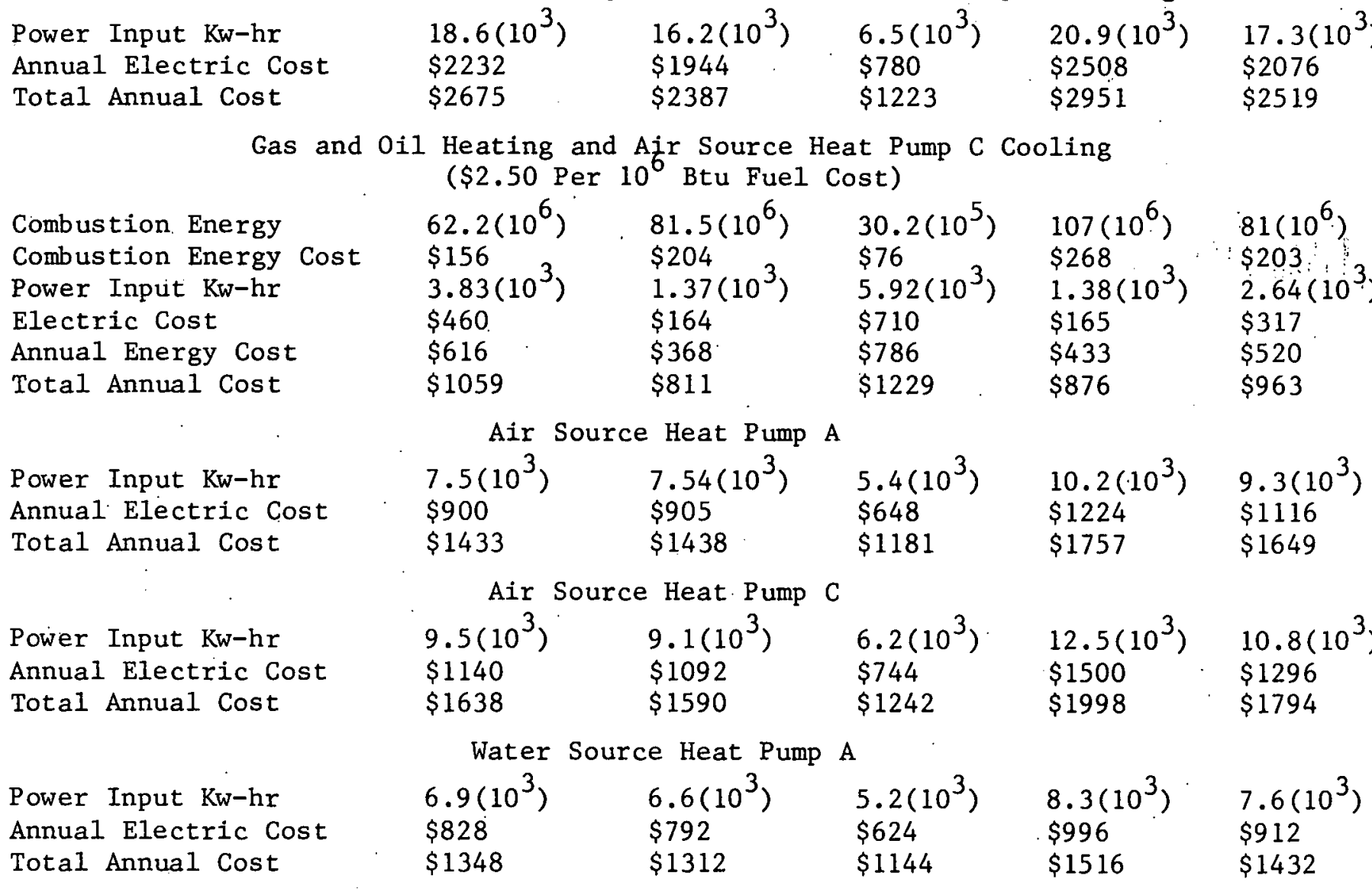

Modified Water Source Heat Pump A With Pre-Water Heat Exchanger -- Alternative I

$\begin{array}{llllll}\text { Power Input Kw-hr } & 6.1\left(10^{3}\right) & 5.9\left(10^{3}\right) & 4.5\left(10^{3}\right) & 7.5\left(10^{3}\right) & 6.8\left(10^{3}\right) \\ \text { Annual Electric Cost } & \$ 732 & \$ 708 & \$ 540 & \$ 900 & \$ 816 \\ \text { Total Annual Cost } & \$ 1287 & \$ 1263 & \$ 1095 & \$ 1455 & \$ 1371\end{array}$

Modified Water Source Heat Pump A With Pre-Water Heat Exchanger -- Alternative II

$\begin{array}{llllll}\text { Power Input Kw-hr } & 4.9\left(10^{3}\right) & 5.9\left(10^{3}\right) & 1.2\left(10^{3}\right) & 7.8\left(10^{3}\right) & 6.1\left(10^{3}\right) \\ \text { Annual Electric Cost } & \$ 588 & \$ 708 & \$ 144 & \$ 936 & \$ 732 \\ \text { Total Annual Cost. } & \$ 1143 & \$ 1263 & \$ 699 & \$ 1491 & \$ 1287\end{array}$

Modified Water Source Heat Pump 'A With Pre-Water Heat Exchanger -- Alternative III

Power Input $\mathrm{Kw}-\mathrm{hr}$.

Annual Electric Cost

Total Annual Cost $4.8\left(10^{3}\right)$
$\$ 576$
$\$ 1131$ $3.4\left(10^{3}\right)$

$\$ 408$

$\$ 963$ $6.2\left(10^{3}\right)$

$\$ 744$

$\$ 1299$ $3.9\left(10^{3}\right)$

$\$ 468$

$\$ 1023$ $4.6\left(10^{3}\right)$

$\$ 552$

$\$ 1107$ 


\section{EXPECTED ENVIRONMENTAL IMPACTS}

All environmental impacts above the ground are expected to be either favorable or of minimal adverse proportions. The largest impact is the decrease in air pollution resulting from the decrease in use of fossil fuels. Some environmental changes may occur in aquifers. Thermal cycling may affect interstitial minerals in aquifers, contaminate ground water, and change ground water temperature thereby accelerating biological growth. None of these effects is expected to be of major consequence.

Since the pumping stations and well housings are designed for the right-of-way next to roads in rural areas similar to electric transformer stations, use of sizable quantities of land surface is not required. In metropolitan areas pumping stations are expected to be under sidewalks with pipelines following the same underground tunnels as other utilities. Thus the loss of the land resource would be inconsequential.

Resources consumed in construction would be lost, yet the savings in energy requirements for the system would provide an offsetting benefit. Electrical energy consumed in the operation of the system would, again, result in a favorable benefit-to-cost ratio when compared with other systems. Failures in the system should be primarily mechanical in nature, although leakages could occur and cause temporary adverse effects. The eventual disposal of system components would have little effect on the environment. Much of the equipment would be salvable.

The greatest effect is to conserve energy and reduce associated environmental effects proportionally. Industrial and urban areas contribute atmospheric contamination not only in the form of particulate and gaseous substances, but also in the form of temperature changes. These 
effects have been shown to modify weather by alteration of rainfall and 13,14
temperature patterns. Reducing energy input and storing thermal energy during warm periods could decrease the adverse effects caused by heat rejection in urban areas. The same would apply to decreased energy usage during cold periods. The reduced use of ofl and gas would more than offset the resources lost in retrieval of energy from aquifers. Contamination of aquifers used for potable water can be avoided. It is anticipated the aquifer system will be confined to a site removed a considerable distance from any other private or public source. In Alabama, health codes allow septic tanks to be placed within $150 \mathrm{ft}$. of potable wells. In addition, the closed system will minimize oxygen intake. Diaphragm-capacitance tanks are recommended to eliminate waterto-air contact. However, the injection of warm water and some air could cause biological growth. Testing biological contamination in the first demonstration projects should be considered to provide a basis for treatment to eliminate this potential problem.

The water table is not significantly affected inasmuch as the same quantity of water is injected as withdrawn. During cooling cycles some water may be discarded to maintain thermal equilibrium.

Of importance could be the precipitation of salts which could clog aquifers and piping. Some salts increase in solubility as temperature drops (mainily carbonates), but others increase in solubility as temperature increases. The temperature range employed in the proposed systems $\left( \pm 32.5^{\circ} \mathrm{F}\right)$ is here considered to be insignificant from a geochemical standpoint. The impact of changing solubility of salts in aquifers, mostly $\mathrm{Ca}$ and $\mathrm{Mg}$ salts, at the' anticipated temperature ranges could be measured only in terms of geologic time. 
The carbonate of calcium is the most predominant carbonate in nature and, obviously, is the primary constituent in limestone or dolomite aquifers. Also, carbonate may be a predominant substance in interstitial spaces in other aquifers, notably sandstones. These considerations should"apply to most aquifers.

Calcium carbonate is dissolved by weak acids by the following:

$$
\mathrm{CaCO}_{3}+\mathrm{H}^{+} \rightarrow \mathrm{Ca}^{++}+\mathrm{HCO}_{3}^{-}
$$

and, limestone dissolves by the equation:

$$
\mathrm{CaCO}_{3}+\mathrm{H}_{2} \mathrm{CO}_{3}=\mathrm{Ca}^{++}+2 \mathrm{HCO}_{3}^{-}
$$

which is an equilibrium situation where the reaction can go in either direction.

$\mathrm{pH}$ is a controlling factor inasmuch as the hydrogen ion concentration affects the equation first noted above. At low $\mathrm{pH}$, the forward action results in solution, whereas at high pIl the reverse action would occur leading to precipitation.

A more dominant situation relates to the pressure of $\mathrm{CO}_{2}$ above the solution. This determines the concentration of dissolved $\mathrm{H}_{2} \mathrm{CO}_{3}$ by the relationship:

$$
\mathrm{H}_{2} \mathrm{O}+\mathrm{CO}_{2} \rightleftharpoons \mathrm{H}_{2} \mathrm{CO}_{3}
$$

Any process increasing the available $\mathrm{CO}_{2}$ promotes dissolution; decreasing the amount of $\mathrm{CO}_{2}$ promotes precipitation.

The above has a bearing on the effects of temperature on the solubility of $\mathrm{CaCO}_{3}$ in natural processes. The solubility of $\mathrm{CaCO}_{3}$ in pure water "...decreases somewhat as the temperature rises." This is opposite to the behavior of most salts 15

to the behavior of most salts.

$\mathrm{CO}_{2}$, like any other gas, is less soluble in hot water than in cold 
water. For instance, in oceans $\mathrm{CaCO}_{3}$ at depth is dissolved, whereas at warm surface temperatures there may be precipitation.

It is considered that a temperature increase of possibly 25 to $35^{\circ} \mathrm{F}$ in return water to an aquifer would not result in any change in the chemical makeup of the circulation water, nor would it affect the physical parameters of the aquifer. The $\mathrm{pH}$ of "pure" water is 7.0 , but if . exposed to the atmosphere for a short time will be near 5.7 as a result of the intake of $\mathrm{CO}_{2}$ from the atmosphere (unless neutralized by other. things). Any process in the pumping and circulation of water in a heat pump system that affects $\mathrm{pH}$ might affect the solubility of both carbonates and salts. This should be minor.

Salts such as $\mathrm{NaCl}, \mathrm{K}_{2} \mathrm{SO}_{4}$, and $\mathrm{Ba}\left(\mathrm{NO}_{3}\right)_{2}$ dissolve in water to form neutral solutions. Others such as iron sulfate may hydrolize leaving an acidic solution. Granted these changes will occur in nature, the introduction of a minimal temperature escalation should have no significant effect on the nature of the aquifer nor that of the circulating water. Silica, the constituent of sandstones, is dissolved by a process of forming a gel--in nature this may require thousands of years to be perceptible. Laboratory studies of the solubility of chemical substances have shown a requirement for high temperature increments over extended periods to realize any effects of temperature. In studies involving the injection of liquid wastes into core samples under simulated reservoir conditions of temperature and pressure--i.e. $200^{\circ} \mathrm{F}$ and $600 \mathrm{psi}$, no difference in the permeability to flow was noted when compared with normal temperatures and pressures (surface conditions). 16 
In summary, any change in aquifers under conditions of nominal increases in temperature of the return water--anything in the range of $25^{\circ}, 35^{\circ}$ or even $50^{\circ}$ is expected to be negligible.

For the most part, environmental changes are expected to be positive; minor adverse effects can be dealt with or justified in light of the beneficial aspects of the system performance. 


\section{PROJECTED GROWTH}

Using the module concept, this version of the heat pump centered integrated community energy system can start in any populated area and expand to cover the entire community. The system is applicable to homes, businesses, major buildings, etc.

In new construction areas, codes can be developed requiring primary water distribution systems and wells. As there is additional population expansion in an area, additional wells can be used to increase capacity. An additional set of wells will not only provide the increased requirements for one module, but also can be used to provide the increased capacity requirements for a number of modules. This type growth results from the specification of 6 -inch lines in the primary water distribution system.

As fuel prices and electricity prices increase, local populated areas can individually convert to this system. Conversion of internal centrally controlled cooling and heating systems is minor. In a home with a furnace and air conditioning, or heat pump, the heat exchangers and blowers in the duct work can be replaced by a water source heat pump. The conversion includes plumbing, pump installation, and minor ducting changes. In larger buildings, most chillers already use water sources from cooling towers. This system replaces the water source with water of more favorable temperature. It also permits some chiller systems to be converted directly to heating, eliminating gas, coal or oil furnaces, or boilers. This conversion can be of appreciable significance.

Additional wells can be added in a number of modules to provide water to areas not located above or near adequate aquifers. Additional pumps would be required in the primary distribution system for the transfer 
of water. Based on the capacity of present urban water distribution systems, the minimum 6-inch lines would be sufficient for most areas. This can be deduced from pressure-loss curves shown in Figures 8 and 9 . Heavily populated areas will require larger, separate mains.

Since the water system will be available to buildings, the development of water-source heat pumps for hot water heating is a possibility. This demand can arise once sufficient systems have been installed. Additional supplementary heating will have to be provided to the overall system to meet the hot water demand. Annual storage allows energy addition at any time of the year. The community energy concept allows other additions such as refrigeration in food and dairy stores, etc.

Growth would be expected to increase as water-source heat pumps become more efficient and some of the identified alternatives are evaluated. The basic system provides for these modifications without major changes. The system has been devised so the projected growth can be similar to expansion in use following the introduction of natural gas into the American economy. Increased natural gas prices and other energy prices could accelerate this process. 


\section{IDENTIFIED VARIATIONS}

A basic concept in using the proposed heat pump system is that essentially $100 \%$ of : the system is comprised of off-the-shelf components. A number of variations may be used to upgrade the performance of the basic. system through heat pump system changes and through different control variations. The first 3 alternatives add a heat exchanger . and new controls. A second set of variations involves the design of basic water distribution systems between aquifers and the ieat pump systems. These systems are not analyzed for performance due to either loss in performance or higher capital andioperating costs. The third set of variations employs abandoned deep mines or caverns for energy storage rather than aquifers.

The first 3 alternatives use a water-to-air (water-to-water) heat exchanger before the water enters the water source heat pump. A schematic of the system is shown in Figure 14.

The water temperature range in the first alternative is the same as that proposed for the basic system, $60^{\circ} \mathrm{F}$ for cooling and $80^{\circ} \mathrm{F}$ for heating. It should be noted that incoming water temperature is below room temperature for cooling and warmer than room temperature for heating. Therefore the air can be either precooled or preheated before reaching the heat pump. As a result some heat transfer occurs without compressor input thereby increasing overall efficiency. The change in air temperature and water temperature reaching the heat pump decreases heat pump efficiency. However, overall system capacity and performance is increased.

Two methods of control are envisioned for this system:

1. The heat pump and water-to-air (water-to-water) heat exchanger operate simultaneously at all times, and 
2. The heat exchanger operates individually for heating until outside temperature reaches a set point and then the waterto-air (water-to-water) heat exchanger and heat pump both operate.

This mode of operation is not effective for cooling since temperatures sufficiently low for dehumidification are required.

Performance tables for this alternative are all based on sumultaneous operation. This system is evaluated for performance and economics in the respective sections (Alternative I).

The second alternative uses a different type of control but uses the same hardware as the first alternative (Figure 14). The water range is $60^{\circ} \mathrm{F}$ to $40^{\circ} \mathrm{F}$ rather than the $80^{\circ} \mathrm{F}$ to $60^{\circ} \mathrm{F}$ range. For heating the performance drops by approximately $10 \%$ as a result of the lower-temperature water. However, for cooling direct heat exchange is available, and the performance more than doubles. Only fan power and pumping power are required; neither the heat pump cycle nor compressor is utilized for cooling except as a possible backup. This system on a long-term basis would conserve the most energy (Alternative II).

A third alternative is to reverse the temperature process and to use $100^{\circ} \mathrm{F}$ to $80^{\circ} \mathrm{F}$ water. In this case the cooling performance with $90^{\circ} \mathrm{F}$ water is decreased; however the performance for heating is appreciably higher. Since all the heating is sensible, higher airflow is required thereby increasing fan power to provide sufficient airflow. The higher temperatures also make waste heat addition more difficult and partially limit the system to areas where cooling load to heating load is sufficient to eliminate waste heat addition (Alternative III).

In addition to the proposed heat pump system, 4 additional heat pump water distribution systems were considered.

The first 2 systems use individual water source heat pumps in the 
controlled environments. The second 2 systems use a central heat pump and transmit chilled and hot water. The piping sequences are shown in Figures 17 to 20 .

The first 2 systems were eliminated due to performance losses associated with some loss of temperature control of the water. Also, successive heat pumps would receive degraded-temperature water. Separate provisions for heating and cooling are not available.

The central heat pump systems are eliminated for the following reasons :

1. There is some question on the realiablity of the required insulation on pipes over a long period of time (in excess of 20 years).

2. Piping costs are appreciably higher (Table 43). Also the average distance water travels is higher requiring larger pipes and/or more pumping power.

3. Operating personnel are required at extra expense.

4. Land must be acquired for central units.

5. Land, building, and central units require more capital than individual units in per-unit output.

6. Performance of central units is not much higher than that of individual units.

An alternative to aquifer storage is storage in abandoned mines or caverns. In these systems separate mines or caverns are required for cold and warm water to prevent energy degradation. In addition, the water probably must be transported greater distances, requiring more pump power, since mines are not available locally in most urbanized areas. However, this is a viable alternative in areas of sub-standard aquifers. 


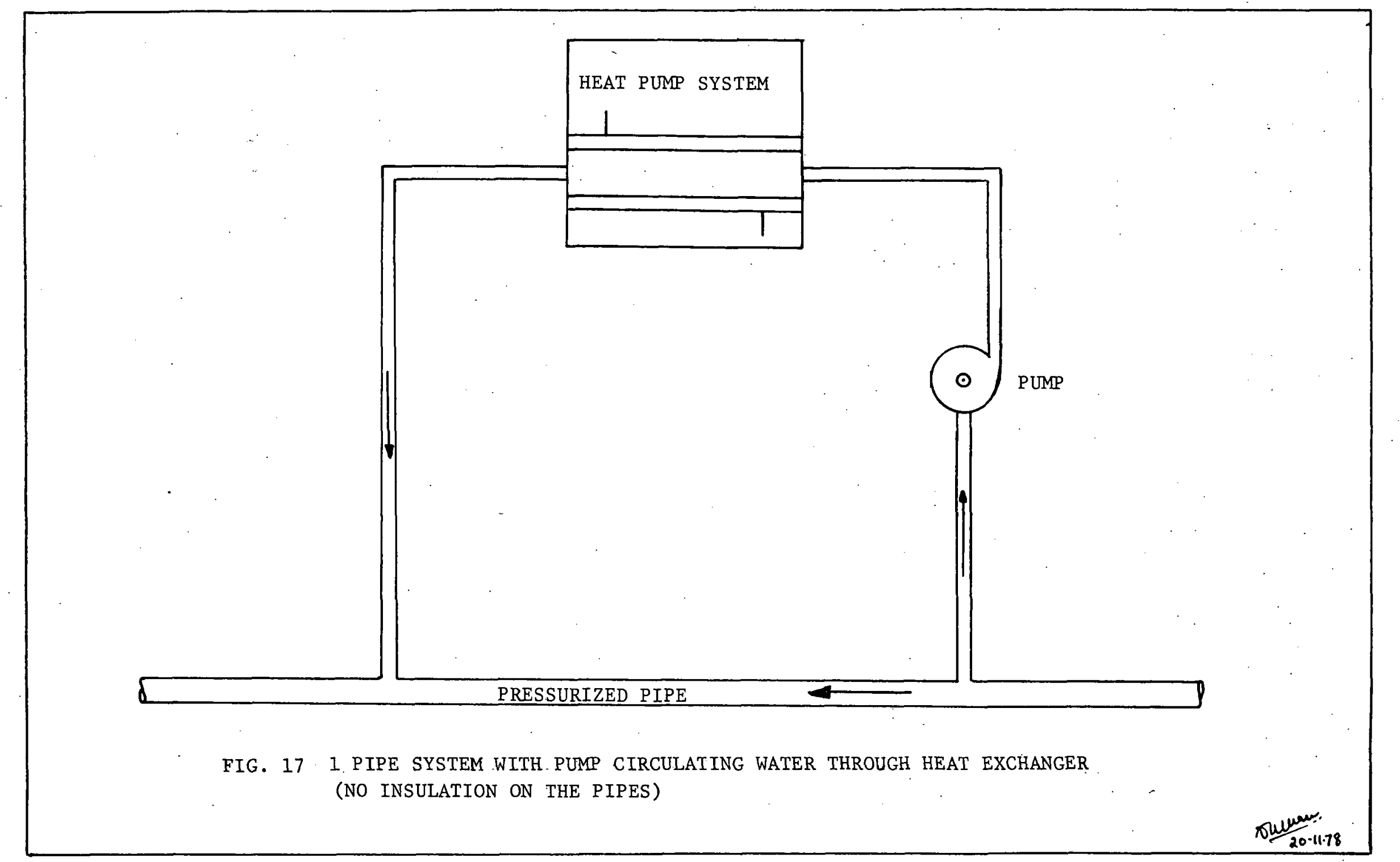




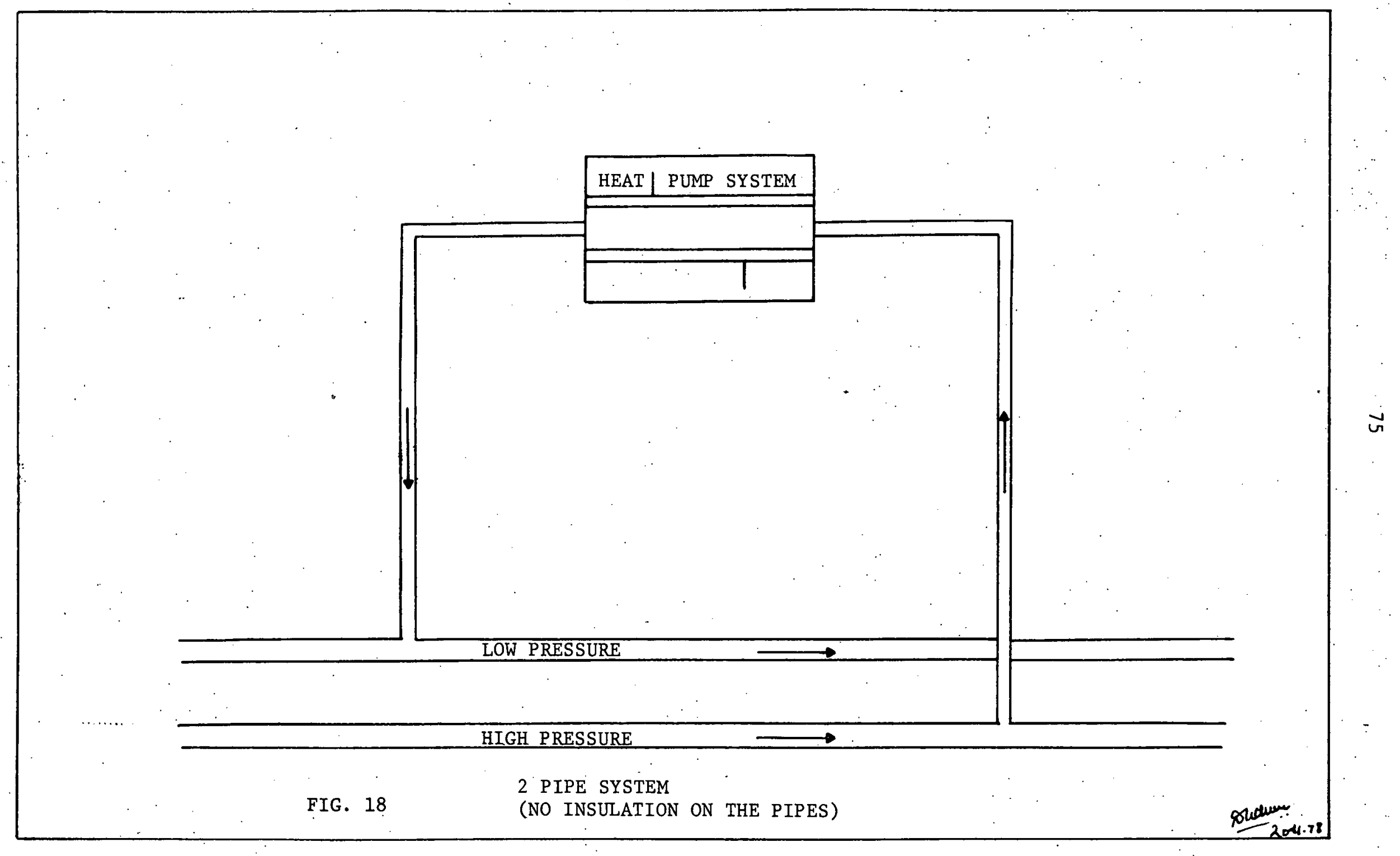




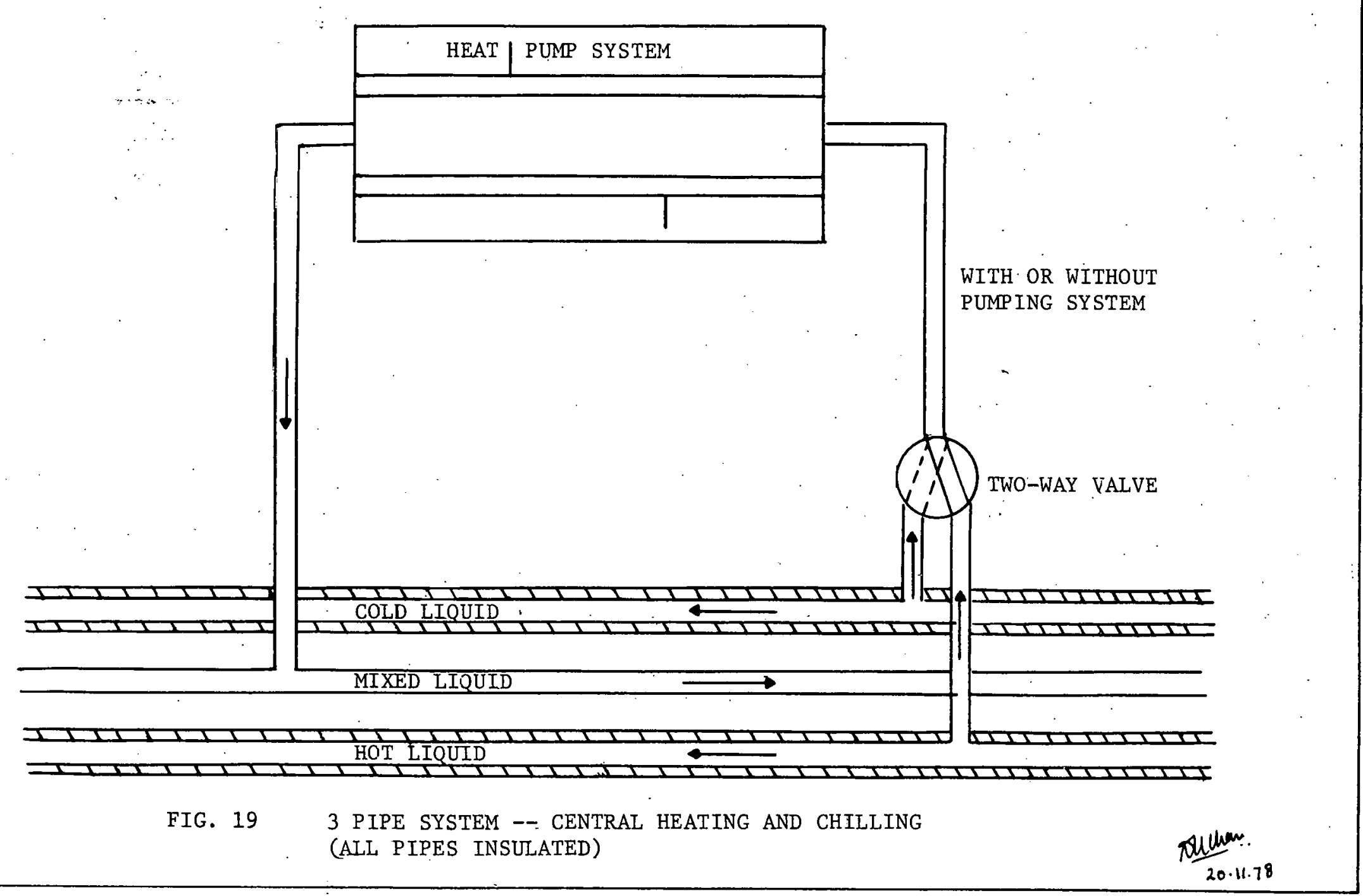




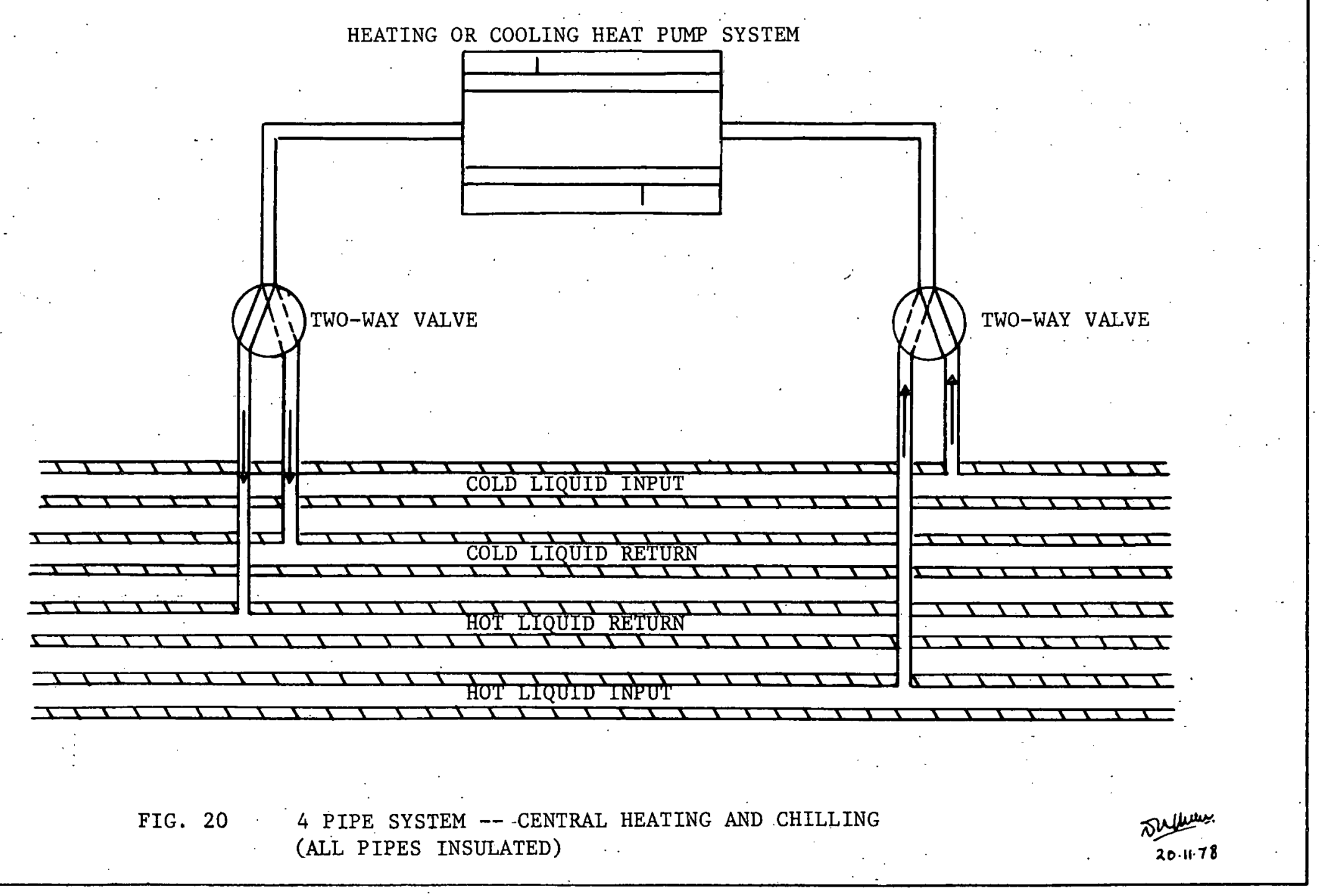




\section{COMPONENT TESTING REQUIREMENTS}

Testing of actual components in the proposed system is not required as all components are off-the-shelf items. Testing of heat-pump systems identified as alternatives and experimentally evaluating a dual-well. aquifer storage system in the laboratory and on a full-scale basis is feasible. Some tests could be conducted on well-water effects on heat exchangers, however appreciable research in this area is already being conducted. An option is to try to influence the direction of some of these projects.

The auxillary direct heat transfer systems in series or parallel with the heat pump show the best performances. Some evaluation tests producing performance data for the first three alternatives is desired.

Some testing should be applied to the aquifer system to determine actual temperature distributions and efficiency of the energy (both hot and cold) return. 2 real possibilities are considered.

1. Tests can be made on a scaled-down sand bed in the laboratory. Dimensionless numbers relating velocities, temperature, energy, porosity, permeability, specific heats, and mass can be developed and tested for applicability.

2. A number of probes should be included in a 2-well demonstration. project to confirm temperature distribution in actual utilization. The buoyancy effect of the warm water is a major question. The results of injection and return from one well system near Mobile, Alabama, has shown an energy efficiency of $59 \%$. 'The proposed 2-well system is expected to have a higher efficiency which will improve as the system attains annual equilibrium.

A smail water source heat pump could be utilized to provide hot and cold water circulation in both laboratory and field testing. 
IX. ANALYSIS DESCRIPTIONS

The following techniques were used to make the analysts in the preceding sections. The sequence corresponds with the sequences in the previous sections.

IX.A. Controlled Environmental System - Heat Pump Performance

The controlled environment includes homes, apartments, businesses, malls, major buildings, etc. A study has been made to show the variations of loads in typical neighborhoods and apartments: In general the loads are directly related to floor area with variations associated with insulation, infiltration, usage, Iocation such as inside apartments, etc. A typical. space of $2000 \mathrm{ft} .^{2}$ has been chosen as a basis for energy evaluations around the country. The insulation values are based on the TVA superinsulator package which also corresponds to the ASHRAE recommendations for most of the northern parts of the country. TVA recommends use of the insulation values throughout Alabama. The basic insulation for this package is:

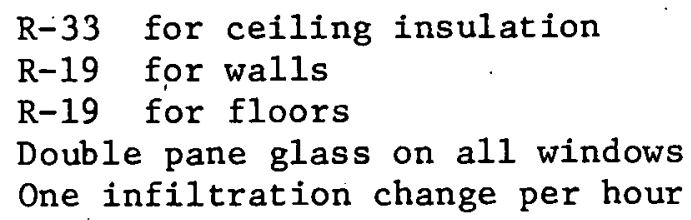

Using the basic ASHRAE load calculation techniques and converting to an annual load using degree-days, the annual heating and cooling loads were calculated for selected cities throughout the country. These are listed in Tables 5 and 6 in the performance section. III. The degree-days are based on an equilibrium temperature of $65^{\circ} \mathrm{F}$, allowing for a normal internal load. The degree-days can be changed to any equilibrium temperature by multiplying the degree change by 365 and subtracting and adding to the corresponding heating and cooling degree-days. For larger buildings equilibrium tempera- 
tures, zero heating or cooling, often move down to the $40^{\circ} \mathrm{F}$ to $50^{\circ} \mathrm{F}$ range. Values are possible in the freezing range.: In these type buildings, the heating and cooling systems are individually designed and can use outside ambient air for cooling at the lower temperatures. Due to variation in systems in each building and most chilling systems are already water-source units, little direct analysis is made of these buildings. It is assumed the new variations in water temperatures will show an increase in performance for most of these systems. In the heating mode most of these buildings can be converted from oil, coal, or gas to heat pumps using much of the same equipment used for cooling. The design change will be of major proportions.

For performance evaluation, not loads, a computer program has been developed to predict annual performance as a function of temperature (Appendix I). The input to the program for 24 cities is NOAA weather data showing average annual hours in $5^{\circ} \mathrm{F}$ temperature increments (Table 22); 99\% design temperatures, internal load and heat pump performance data (Tables 23 to 25); and the heating and cooling loads at ASHRAE design temperatures. The loads are matched to heat pump capacities rather than actual loads. The output is a measure of performance only. The internal load allows the program to be used for larger buildings. Heating loads above heat-pump capacity are meet with the addition of resistance heating. Runs were made for a variation of heat pumps. The first 2 studies with the standard internal load of 5000 Btu use air source heat pumps A and $\mathrm{C}$ to indicate performance with high and normal performance pumps with additional resistance heating (Tables 26 and 27 ). The use of twin units doubles pump capacities of air source heat pumps $A$ and $C$ which have been 


\begin{tabular}{|c|c|c|c|c|c|c|c|c|c|c|c|c|c|c|c|c|c|c|c|c|c|c|c|c|c|c|c|c|}
\hline thes, s & -18 & -13 & -8 & -3 & 2 & - & 12 & 17 & 22 & 27 & 32 & 37) & 42 & 47 & 52 & 57 & 62 & 67 & 72 & 77) & 82 & 87 & 92 & 97 & 102 & 107 & 112 & 117 \\
\hline 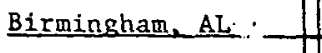 & of & 0 & 0 & 0 & 0 & 0 & 3 & 6 & 17 & 69 & 143 & 292 & 433 & 528 & 614 & 668 & 742 & 805 & 908 & 1138 & 924 & 658 & 488 & 264 & 62 & 5 & 0 & 0 \\
\hline 1 scon, $\mathrm{AZ}$ & of & $\underline{0}$ & 0 & 0 & 0 & of & 0 & 0 & 0 & 2 & 25 & 98. & 248 & 417 & 598 & 716 & 800 & 763 & 781 & 870 & 959 & 777 & 656 & 520 & 357 & 152 & 31 & 0 \\
\hline s Angeles, CA & 0 & 0 & 0 & 0 & 0 & of & 0 & 0 & 0 & 0 & 0 & of & 10 & 107 & 428 & 1054 & 1904 & 2193 & 1654 & 881 & 380 & 117 & 28 & 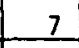 & 4 & 1 & 0 & 0 \\
\hline enver, co & 1 & 0 & 1 & 6 & 22 & 36 & 78 & 119 & 216 & 359 & 553 & 721 & 717 & 692 & 704 & 678 & 731 & 783 & 684 & 549 & 437 & 332 & 236 & 103 & 10 & 1 & 0 & 0 \\
\hline iami, FL & 0 & $\underline{0}$ & 0 & 0 & 0 & 0 & 0 & 0 & 0 & 0 & .0 & of & 4 & 26 & 71 & 147 & 277 & 452 & 810 & 1708 & 2463 & 795 & 888 & 125 & 2 & 0 & 0 & 0 \\
\hline Atlanta, GA & 0 & $\underline{0}$ & 0 & 0 & 0 & 0 & $\underline{2}$ & 8 & 19 & 44 & 112 & 271 & 468 & 598 & 676 & 735 & 784 & 823 & 926 & 1185 & 883 & 625 & 401 & 177 & 29 & 2 & 0 & 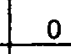 \\
\hline Hilo, & 0 & 0 & 0 & 0 & 0 & 0 & 0 & 0 & 0 & 0 & 0 & 0 & 0 & 0 & 0 & $\underline{0}$ & 11. & 361 & 2272 & 3001 & 2126 & 975 & 24 & 0 & $\underline{0}$ & $\underline{0}$ & 0 & 0 \\
\hline Chicago, IL & 0 & 0 & 3 & 12 & 25 & 59 & 85 & 117 & 19.6 & 335 & 551 & 822 & 800 & 591 & 543 & 569 & 592 & 653 & 769 & 762 & 563 & 370 & 220 & 105 & 27 & 2 & 0 & $\underline{0}$ \\
\hline New Orleans, LA & 0 & 0 & 0 & 0 & 0 & 0 & 0 & 0 & 0 & 2 & 9 & 47 & 128 & 282 & 449 & 621 & 692 & 850 & 987 & 1189 & 1671 & 979 & 620 & 229 & 12 & 0 & 0 & 0 \\
\hline IA & 0 & 0 & 0 & 1 & 3 & 11 & 35 & 75 & 149 & 254 & 431 & 669 & 851 & 831 & 756 & $765^{\circ}$ & 783 & 805 & 819 & 676 & 433 & 45 & 127 & 39 & 10 & 0 & 0 & 0 \\
\hline etroit, MI & 0 & 0 & 0 & 1 & 4 & 17 & 61 & 131 & 248 & 7 & 618 & 884 & 808 & 595 & 566 & 592 & 633 & 695 & 783 & 721 & 516 & 314 & 148 & 47 & 9 & 0 & $v$ & 0 \\
\hline aint Louis, & 0 & $\underline{0}$ & 0 & 1 & 7 & 15 & 40 & 77 & 134 & 212 & 411 & 650 & 671 & 620 & 578 & 585 & 575 & 646 & 728 & 823 & 763 & 579 & 376 & 198 & 65 & 13 & 1 & 1 \\
\hline New York, NY. & 0 & 0 & 0 & 0 & 0 & 1 & 10 & 26 & 96 & 188 & 330 & 603 & 858 & 838 & 796 & 722 & 745 & 754 & 877 & 926 & 604 & 263 & 96 & 28 & 5 & 0 & 0 & 0 \\
\hline kron, $\mathrm{OH}$ & 0 & $\underline{0}$ & $\underline{0}$ & 5 & 11 & 32 & 67 & 153 & 226 & 429 & 646 & 867 & 657 & 630 & 616 & 633 & 686 & 778 & 839 & 675 & 437 & 275 & 112 & 24 & 3 & 0 & 0 & 0 \\
\hline ulsa, OK & 0 & 0 & 0 & 0 & 1 & 2 & 12 & 29 & 75 & 59 & 265 & 438 & 535 & 611 & 637 & 622 & 636 & 71 & 752 & 838 & 816 & 649 & 481 & 333 & 149 & 43 & 10 & 1 \\
\hline ortland, 0 R & 0 & 0 & 0 & $\underline{u}$ & 0 & of & 1 & 4 & 10 & 40 & 123 & 343 & 772 & 238 & 1271 & 1274 & 1316 & 1001 & 581 & 373 & 211 & 126 & 54 & 23 & 6 & 1 & 0 & 0 \\
\hline hiladelphia, PA & 0 & 0 & 0 & 0 & 0 & of & 9 & 32 & 100 & 189 & 335 & 654 & 818 & 758 & 701 & 663 & 710 & 735 & 809 & 863 & 655 & 420 & 225 & 74 & 17 & 1 & 0 & 0 \\
\hline rovidence, RI & 0 & 0 & 0 & 1 & 4 & 9 & 39 & 84 & 163 & 275 & 491 & 729 & 829 & 790 & 763 & 762 & 764 & 822 & 799 & 668 & 434 & 229 & 86 & 25 & $\underline{2}$ & $\underline{0}$ & 0 & $\underline{0}$ \\
\hline Columbia, SC & 0 & 0 & $\underline{0}$ & 0 & 0 & 0 & 0 & 2 & 19 & 56 & 138 & 293 & 411 & 523 & 623 & 673 & 22 & 808 & 895 & 1113 & 940 & 657 & 489 & 301 & 92 & 11 & 1 & 0 \\
\hline ashville, TN & 0 & $\underline{0}$ & 0 & 1 & 1 & 3 & 9 & 28 & 67 & 132 & 263 & 463 & 565 & 627 & 619 & 637 & 697 & 738 & 838 & 933 & 814 & 582 & 443 & 227 & 66 & 11 & 1 & 0 \\
\hline ouston, TX & 0 & 0 & 0 & 0 & 0 & 0 & 0 & 0 & 2 & 4 & 28 & 64 & 141 & 291 & 452 & 570 & 681 & 772 & 980 & 1172 & 1611 & 949 & 677 & $327^{\circ}$ & 56 & 1 & 0 & 0 \\
\hline 1t Lake City, UT & 0 & $\underline{0}$ & 0 & 0 & 2 & 16 & 41 & 80 & 1.58 & 328 & 564 & 798 & 831 & 755 & 685 & 682 & 635 & 614 & 615 & 569 & 447 & 368 & 309 & 196 & 66 & 8 & 0 & 0 \\
\hline eattle, WA & 0 & 0 & 0 & 0 & 0 & 0 & 0 & 3 & 20 & 39 & 104 & 427 & 914 & 408 & 1445 & 1462 & 1272 & 750 & 448 & 258 & 123 & 62 & 24 & 6 & $=$ & 0 & 0 & 0 \\
\hline 12wajice, $K I$ & 1 & 2 & 4 & 18 & 47 & 83 & 116 & 276 & 285 & 421 & 659 & 913 & 774 & 611 & 591 & 585 & 634 & 710 & 753 & 597 & 390 & 226 & 96 & 32 & 6 & 0 & 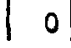 & 0 \\
\hline
\end{tabular}

* From Sumary of Hour1y Observations, NOAA, Separate Number For Each C1ty, 10 Year Average 
TABLE 23. PERFORMANCE OF AIR-SOURCE HEAT PUMP A*

COOLING

\begin{tabular}{|c|c|c|c|c|c|c|c|}
\hline $\mathrm{DBT}^{1}$ & $\mathrm{WBT}^{2}$ & $\begin{array}{c}\text { TOTAL CAP } \\
\text { MBTUH } \\
\end{array}$ & $\begin{array}{l}\mathrm{SE} \\
72 \\
\end{array}$ & $\begin{array}{l}\text { 3LE CAP } \\
76 \\
\end{array}$ & $\begin{array}{c}\text { IDBT }^{3} \\
80 \\
\end{array}$ & $\begin{array}{c}\text { INPUT POWER }{ }^{4} \\
\mathrm{KW} \\
\end{array}$ & $\mathrm{COP}^{5}$ \\
\hline 85 & $\begin{array}{l}59 \\
63 \\
67 \\
71\end{array}$ & $\begin{array}{l}33.1 \\
34.9 \\
36.7 \\
38.5\end{array}$ & $\begin{array}{l}28.6 \\
23.1 \\
18.0 \\
13.3\end{array}$ & $\begin{array}{l}33.2 \\
27.5 \\
22.2 \\
17.2\end{array}$ & $\begin{array}{l}34.2 \\
32.0 \\
26.4 \\
21.2\end{array}$ & $\begin{array}{l}3.93 \\
4.03 \\
4.13 \\
4.23\end{array}$ & $\begin{array}{l}2.47 \\
2.54 \\
2.61 \\
2.67\end{array}$ \\
\hline 95 & $\begin{array}{l}59 \\
63 \\
67 \\
71 .\end{array}$ & $\begin{array}{l}31.4 \\
33.4 \\
35.4 \\
37.4\end{array}$ & $\begin{array}{l}28.3 \\
22.8 \\
17.6 \\
12.9\end{array}$ & $\begin{array}{l}31.8 \\
27.4 \\
22.0 \\
17.0\end{array}$ & $\begin{array}{l}33.1 \\
32.1 \\
26.4 \\
21.1\end{array}$ & $\begin{array}{l}4.23 \\
4.33 \\
4.43 \\
4.43\end{array}$ & $\begin{array}{l}2.19 \\
2.26 \\
2.34 \\
2.48\end{array}$ \\
\hline 105 & $\begin{array}{l}59 \\
63 \\
67 \\
71\end{array}$ & $\begin{array}{l}29.1 \\
31.3 \\
33.5 \\
35.7\end{array}$ & $\begin{array}{l}27.6 \\
22.2 \\
17.1 \\
12.3\end{array}$ & $\begin{array}{l}30.1 \\
27.0 \\
21.7 \\
16.6\end{array}$ & $\begin{array}{l}31 . .5 \\
31.5 \\
26.3 \\
21.0\end{array}$ & $\begin{array}{l}4.43 \\
4.53 \\
.4 .63 \\
4.73\end{array}$ & $\begin{array}{l}1.93 \\
2.03 \\
2.12 \\
2.21\end{array}$ \\
\hline 115 & $\begin{array}{l}59 \\
63 \\
67 \\
71\end{array}$ & $\begin{array}{l}26.4 \\
28.7 \\
31.1 \\
33.4\end{array}$ & $\begin{array}{l}26.5 \\
21.4 \\
16.4 \\
11.5\end{array}$ & $\begin{array}{l}28.0 \\
26.4 \\
21.1 \\
16.0\end{array}$ & $\begin{array}{l}29.5 \\
29.5 \\
25.9 \\
20.7\end{array}$ & $\begin{array}{l}4.73 \\
4.83 \\
4.93 \\
5.03\end{array}$ & $\begin{array}{l}1.64 \\
1.74 \\
1.85 \\
1.95\end{array}$ \\
\hline
\end{tabular}

60

70

75

80

\begin{tabular}{|c|c|c|c|c|c|c|c|c|c|}
\hline \multirow[b]{2}{*}{$\begin{array}{l}\text { IDBT } \\
\text { ODT } \\
\end{array}$} & & & & \multicolumn{2}{|r|}{80} & \multirow[b]{2}{*}{$\mathrm{COP}^{5} @ 7$} \\
\hline & $\begin{array}{r}\mathrm{CAP} \\
\mathrm{MBTUH} \\
\end{array}$ & $\mathrm{KW}^{4}$ & $\begin{array}{c}\text { CAP } \\
\text { MBTUH }\end{array}$ & $\mathrm{KW}^{4}$ & $\begin{array}{c}\text { CAP } \\
\text { MBTUH } \\
\end{array}$ & $\mathrm{KW}^{4}$ & $\begin{array}{c}\text { CAP } \\
\text { MBTUH } \\
\end{array}$ & $\mathrm{KW}^{4}$ & \\
\hline-8 & 12.0 & 2.6 & 11.0 & 2.7 & 10.5 & 2.7 & 10.0 & 2.8 & 1.19 \\
\hline 2 & 16.0 & 2.8 & 14.7 & 2.9 & 14.1 & 3.0 & 13.5 & 3.1 & 1.49 \\
\hline 12 & 20.7 & 3.1 & 19.2 & 3.2 & 18.4 & 3.3 & 17.7 & 3.3 & 1.76 \\
\hline 22 & 26.1 & 3.3 & 24.4 & 3.5 & 23.5 & 3.6 & 22.6 & 3.6 & 2.04 \\
\hline 32 & 32.0 & 3.6 & 30.1 & 3.7 & 29.1 & 3.8 & 28.1 & 3.9 & 2.38 \\
\hline 42 & 37.9 & 3.8 & 35.9 & 4.0 & 34.8 & 4.1 & 33.8 & 4.2 & 2.63 \\
\hline 52 & 42.9 & 4.1 & 40.8 & 4.3 & 39.7 & 4.4 & 38.7 & 4.5 & 2.78 \\
\hline 62 & 45.8 & 4.3 & 43.9 & 4.6 & 42.9 & 4.7 & 41.9 & 4.8 & 2.80 \\
\hline 72 & 46.3 & 4.5 & 44.6 & 4.8 & 43.8 & 4.9 & 43.0 & 5.0 & 2.72 \\
\hline
\end{tabular}

${ }^{1}$ Outdoor Dry Buib Temperature, ${ }^{\circ} \mathrm{F}$

${ }^{2}$ Indoor wet Bulb Temperature, ${ }^{\circ} F$

${ }^{3}$ Indoor Dry Bulb Temperature, ${ }^{\circ} \mathrm{F}$

${ }^{4}$ Power requirement for compressor, outdoor fan, and indoor fan, $\mathrm{KW}$

${ }^{5}$ Coefficient of Performance $=$ Capacity $(\mathrm{Kw}) /$ Input Power ${ }^{4}(\mathrm{Kw})$

*Based on G.E. Pub. No. 22-1009-6, Apr11 1977 for BWR936A1-A @ $1200 \mathrm{cfm}$ Cost $\$ 2500$ installed per J.E. Christian, Unitary Air-to-Air Heat Pumps, ANL/CES/TE77-10. ( $\$ 300$ surcharge added...for .high performance system making system installed cost $\$ 2800$ for cost comparison) 
TABLE 24. PERFORMANCE OF AIR-SOURCE HEAT PUMP B*

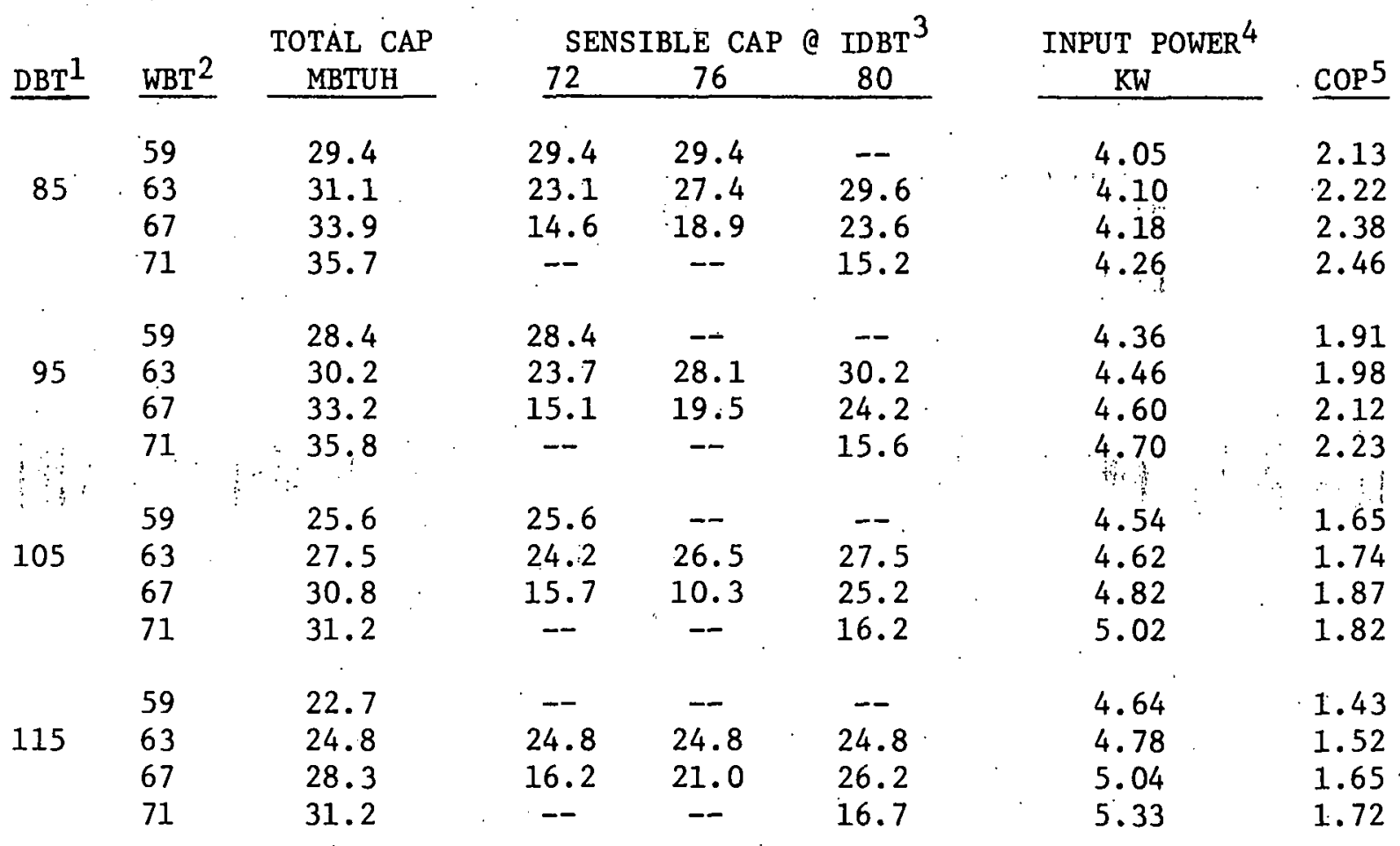

HEATING

\begin{tabular}{|c|c|c|c|c|c|c|c|c|c|}
\hline \multirow{2}{*}{$\begin{array}{l}\text { IDBT } \\
\text { ODT } \\
\end{array}$} & \multicolumn{2}{|c|}{60} & \multicolumn{2}{|c|}{70} & \multicolumn{2}{|c|}{75} & \multicolumn{2}{|c|}{80} & \multirow[b]{2}{*}{$\mathrm{CDP} @ 70^{\circ}$} \\
\hline & CAP & $\mathrm{KW}$ & CAP & $\mathrm{KW}$ & CAP & $\mathrm{KW}$ & CAP & $\mathrm{KW}$ & \\
\hline-18 & 10.7 & 2.46 & 10.4 & 2.46 & 10.4 & 2.46 & 9.4 & 2.46 & 1.24 \\
\hline-8 & 12.0 & 2.66 & 11.5 & 2.66 & 10.9 & 2.66 & 10.5 & 2.66 & 1.27 \\
\hline 2 & 15.5 & 2.86 & 14.7 & 2.86 & 14.0 & 2.88 & 13.5 & 2.91 & 1.51 \\
\hline 12 & 19.0 & 3.06 & 18.2 & 3.06 & 17.5 & 3.11 & 17.0 & 3.16 & 1.74 \\
\hline 22 & 23.5 & 3.26 & 22.0 & 3.31 & 21.5 & 3.34 & 21.0 & 3.36 & 1.95 \\
\hline 32 & 27.5 & 3.41 & 26.6 & 3.51 & 25.7 & 3.56 & 25.0 & 3.61 & 2.22 \\
\hline 42 & 34.0 & 3.66 & 32.2 & 3.76 & 31.3 & 3.79 & 30.5 & 3.86 & 2.51 \\
\hline 52 & 40.5 & 3.86 & 39.0 & 3.96 & 36.3 & 4.04 & 37.5 & 4.11 & 2.89 \\
\hline 62 & 44.5 & 4.01 & 43.7 & 4.21 & 42.8 & 4.28 & 42.0 & 4.36 & 3.04 \\
\hline 72 & -- & -- & 45.9 & -4.31 & 45.7 & 4.41 & 45.5 & 4.51 & 3.12 \\
\hline
\end{tabular}

* Based on York Application Dața Form 515.21-AD2 For CHPO-36 @ 1200 CFM Cost $\$ 2,500$ installed per J.E. Christian, Unitary Air to Air Heat Pumps, $\mathrm{ANL} / \mathrm{CSE} / \mathrm{TE} 77-10$ 
TABLE 25. PERFORMANCE OF AIR-SOURCE HEAT PUMP C*

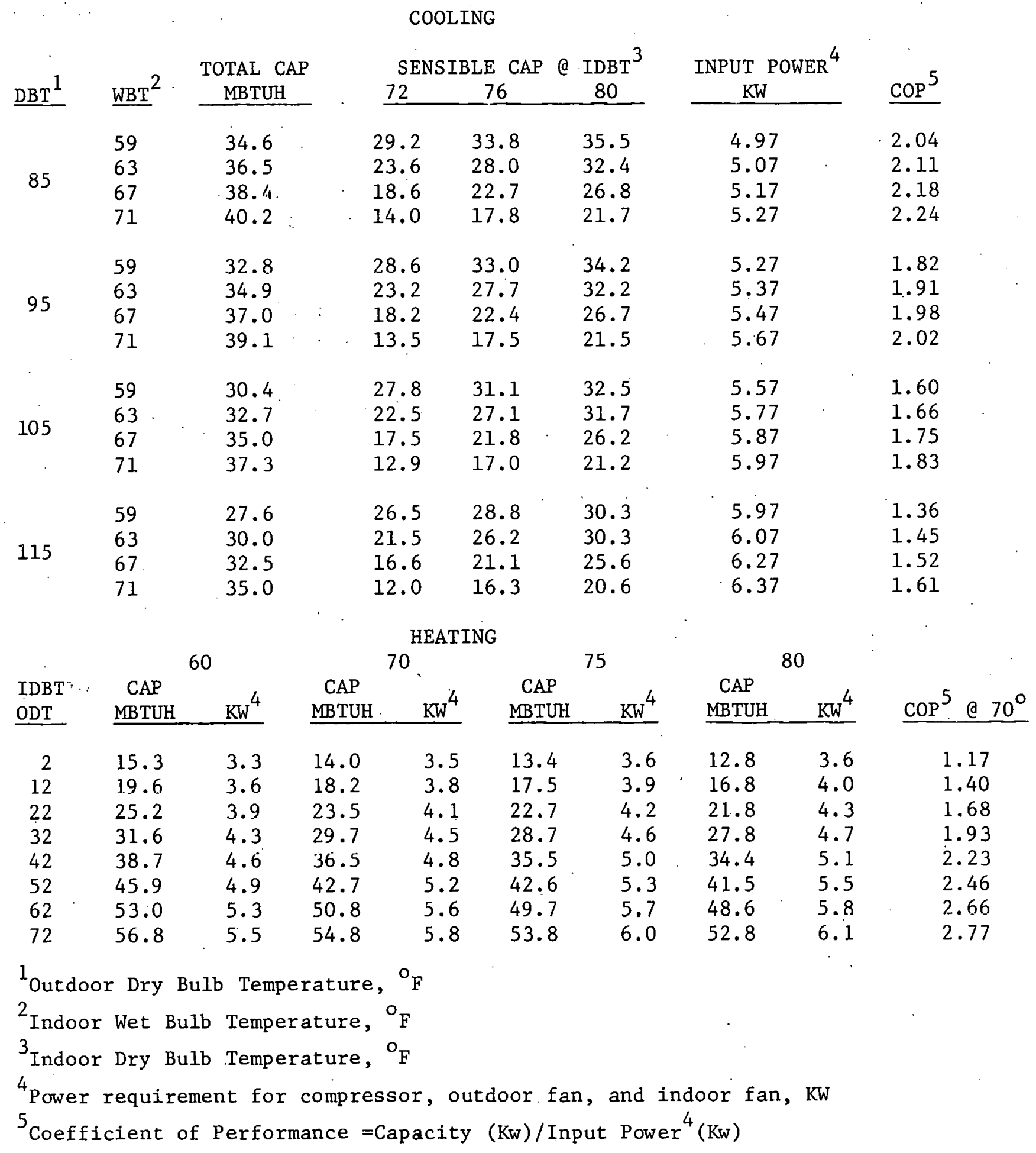

*Based on G.E. Pub. No. 22-1028-6, Oct. 1977, for BWC036B @ $1200 \mathrm{cfm}$ Cost $\$ 2,500$ installed per J. E. Christian, Unitary Air-to-Air Heat Pumps, ANL/CES/TE77-10 
Table 26. Annual. Performance for Temperature Variation in Twenty-four Cities

Air Source Heat Pump A

Internal Load of 5,000 BTUH

Indoor Design Temp. of $70^{\circ} \mathrm{F}$

\begin{tabular}{|c|c|c|c|c|c|c|c|c|c|}
\hline City, State & $\begin{array}{l}\text { Cooling } \\
\text { Design } \\
\text { Temp }{ }_{F}\end{array}$ & $\begin{array}{l}\text { Heating } \\
\text { Design } \\
\text { Temp F }\end{array}$ & $\begin{array}{l}\text { Annual } \\
\text { Heating } \\
\text { Load } \\
\text { BTUx } 10^{-5}\end{array}$ & $\begin{array}{l}\text { Annual } \\
\text { Cooling } \\
\text { Load } \\
\text { BTUx10 }\end{array}$ & $\begin{array}{r}\mathrm{CoO} \\
\mathrm{KWH}^{1} \\
\end{array}$ & $\mathrm{COP}^{3}$ & $\mathrm{KWH}^{1}$ & $\begin{array}{l}\text { Heatin } \\
\mathrm{KWH}^{2} \\
\end{array}$ & $\mathrm{COP}^{3}$ \\
\hline Birmingham, AL & 96 & 17 & 295 & 831 & 8928 & 2.73 & 3496 & 66 & 2.43 \\
\hline Tuscon, AZ & 104 & 28 & 260 & 964 & 10791 & 2.62 & 2946 & 8 & 2.58 \\
\hline Los Angeles, CA & 83 & 41 & 314 & 528 & 5378 & 2.88 & 3388 & 0 & 2.71 \\
\hline Denver, CO & 89 & -5 & 442 & 484 & 5155 & 2.75 & 5701 & 438 & 2.11 \\
\hline Miami, FL & 91 & 44 & 58 & 1763 & 18816 & 2.75 & 633 & 1 & 2.70 \\
\hline Atlanta, GA & 94 & . 17 & 299 & 789 & 8400 & 2.75 & 3510 & 71 & 2.45 \\
\hline Hilo, HI & 84 & 61 & 28 & 1835 & 18984 & 2.83 & 302 & 0 & 2.74 \\
\hline Chicago, II & 94 & -5 & 450 & 500 & 5307 & 2.76 & 5770 & 569 & 2.08 \\
\hline New Orleans, LA & 93 & 29 & 185 & 1159 & 12382 & 2.74 & 2070 & 8 & 2.61 \\
\hline Boston, MA: & 91 & 6 & 490 & 403 & 4219 & 2.80 & 6083 & 323 & 2.24 \\
\hline Detroit, MI & 91 & 3 & 544 & 455 & 4778 & 2.79 & 6958 & 429 & 2.16 \\
\hline Saint Louis, MO & 97 & 2 & 391 & 655 & 7054 & 2.72 & 4906 & 295 & 2.20 \\
\hline Akron, $\mathrm{OH}$ & 89 & 1 & 533 & 422 & 4404 & 2.81 & 6837 & 532 & 2.12 \\
\hline Tulsa, OK & 101 & 8 & 330 & 741 & 8110 & 2.68 & 4049 & 139 & 2.31 \\
\hline Portland, OR & 89 & 17 & 485 & 231 & 2408 & 2.81 & 5631 & 36 & 2.51 \\
\hline Philadelphia, - PA & 93 & 10 & 453 & 550 & 5807 & 2.77 & 5552 & 167 & 2.32 \\
\hline Providence, $\mathrm{RI}$ & 89 & 5 & 501 & 393 & 4088 & 2.82 & 6265 & 314 & 2.23 \\
\hline Columbia, SC. & 97 & 20 & 313 & 836 & 9016 & 2.72 & 3693 & 78 & 2.44 \\
\hline Nashville, TN & 97 & 9 & 338 & 718 & 7726 & 2.72 & 4118 & 152 & 2.32 \\
\hline Houston, TX & 96 & 27 & 179 & 1117 & 12017 & 2.73 & 2024 & 16 & 2.58 \\
\hline Salt Lake City, UT.. & 97 & 3 & 500 & 483 & 5232 & 2.71 & 6307 & 289 & 2.22 \\
\hline Seattle, WA $\cdot \cdot$. & 84 & 21 & 629 & 186 & 1906 & 2.87 & 7266 & 88 & 2.51 \\
\hline Milwaukee, WI & 90 & -8 & 507 & 395 & 4103 & 2.83 & 6633 & 744 & 2.02 \\
\hline
\end{tabular}

Cooling Design Load of 30,000 BTUH at Cooling Design Temperature Heating Design Load of 36,000 BTUH at Heating Design Temperature

1.. Annual energy input to heat pump, includes compressor, outdoor fan and indoor fan

2. Annual energy input to resistance heater

3. Annual cooling or heating load/annual energy input (dimensionless) 
Table 27. Annual Performance for Temperature Variation in Twenty-four Cities

Air-Source Heat Pump C

Internal Load of 5,000 BTHH

Indoor Design Temp. of $70 \mathrm{~F}$

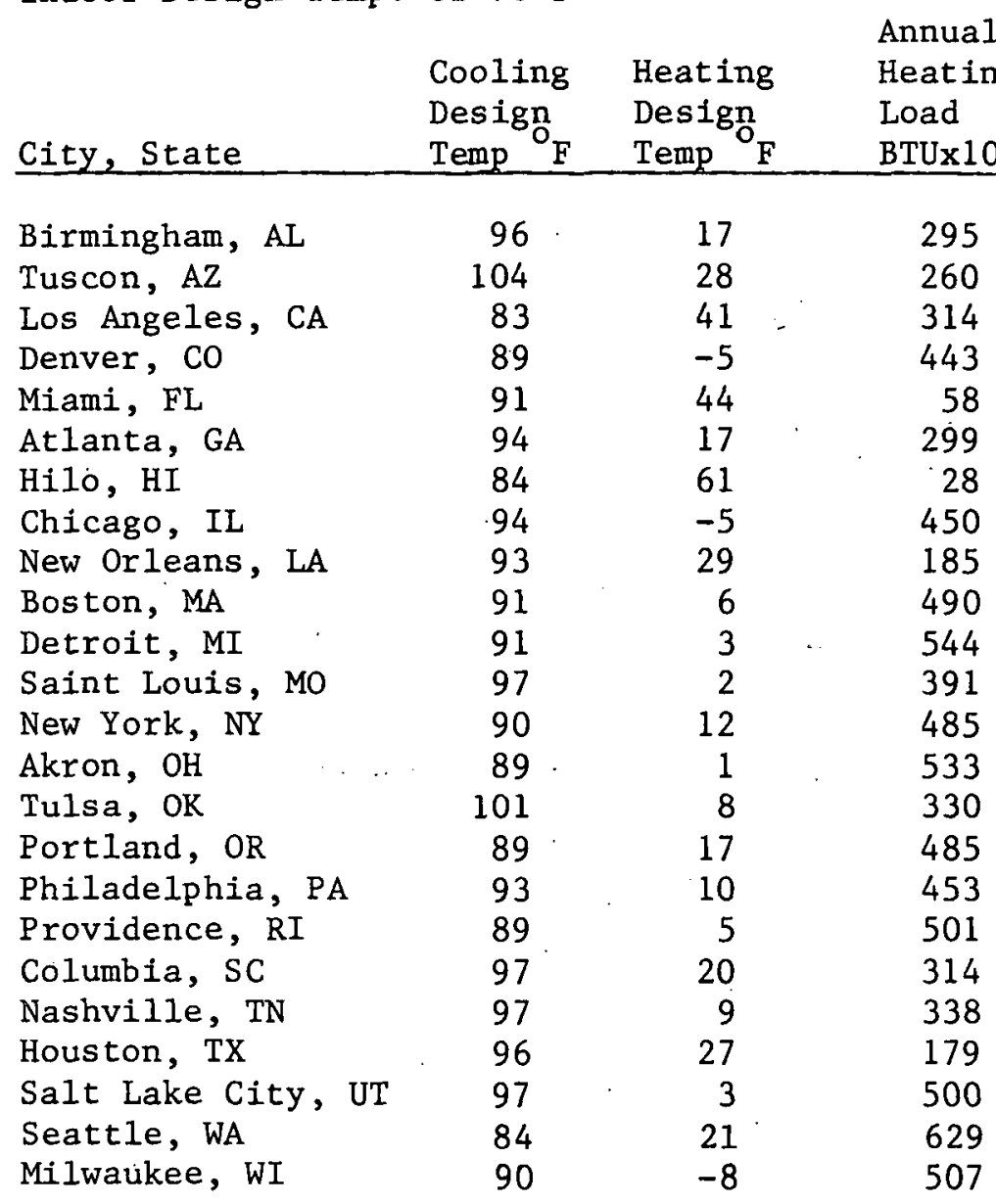

Cocling Design Load of 30,000 BTUH at Cooling Design Temperature Heating Design Load of 36,000 BTUH at Heating Design Temperature

1. Annual energy input to heat pump, includes compressor, outdoor fan and indoor fan

2. Annual energy input to resistance heater

3. Annual cooling or heating load/annual energy input (dimensionless) 
employed to indicate the performance effects of decreased resistance heating (Tables 28 and 29). Since the resistance heat is used a very. small percentage of the time, it may be noted that differences between single and double units are smal1. Obviously these combinations are not economically feasible and have been eliminated from further study. The next 2 studies use the twin unit A with increased internal load. These studies (Tables 30 and 31 ) give the performance effect of larger buildings where the ratio of internal load to maximum external load becomes larger. It has the effect of increasing the cooling load and decreasing the heating load. This variation can be used to match buildings in modules to obtain equal cooling and heating water requirements.

Individual run sheets are shown in Tables 32 to 38 . Tables 32 to 34 demonstrate the variations for air source heat pump A at different locations using a standard internal load of 5000 Btu. Table 35 represents. a sun with the water source heat pump A. Tables 36 and 37 give the performance with larger internal loads to represent the performance for larger buildings where the ratio of internal load to maximum internal load becomes larger. It has the effect of increasing the cooling load and decreasing the heating load. This variation can be used to match buildings in modules to obtain equal cooling and heating energy requirements.

Use of heating oil with an API gravity of 26 , which corresponds to $a^{12}$ specific gravity of 0.9 , results in higher heating value of the fuel or

$$
\begin{aligned}
\mathrm{HHV} & =19,000 \mathrm{Btu} / \mathrm{Ib} \\
& =145,000 \mathrm{Btu} / \mathrm{gal} .
\end{aligned}
$$

These figures are used in the evaluation of gallons of oil required for heating.

The major difference in price between the small unit water source 
Table 28. Annual Performance for Temperature Variation in-Twenty-four Cities

Twin Air Source Heat Pump A Internal Load of 5,000 BTUH Indoor Design Temp. of $700^{\circ}$
Cooling Design Load of 30,000 BTUH at Cooling Design Temperature Heating Design Load of 36,000 BTUH at Heating Design Temperature

\begin{tabular}{|c|c|c|c|c|c|c|c|c|c|}
\hline City, State & $\begin{array}{l}\text { Cooling } \\
\text { Design } \\
\text { Temp of } \\
\end{array}$ & $\begin{array}{l}\text { Heating } \\
\text { Design } \\
\text { Temp O }{ }^{\circ}\end{array}$ & $\begin{array}{l}\text { Annual } \\
\text { Heating } \\
\text { Load } \\
\text { BTUx } 10^{-5} \\
\end{array}$ & $\begin{array}{l}\text { Annual } \\
\text { Cooling } \\
\text { Load } \\
\text { BTU } 10^{-5} \\
\end{array}$ & $\mathrm{KWH}^{\mathrm{l}}$ & $\mathrm{COP}^{3}$ & $\mathrm{KWH}^{1}$ & $\begin{array}{l}\text { Heat ir } \\
\mathrm{KWH}^{2} \\
\end{array}$ & $\mathrm{COP}^{3}$ \\
\hline Birmingham, $\mathrm{AL}$ & 96 & 17 & 295 & 836 & 8989 & 2.72 & 3533 & 1 & 2.45 \\
\hline Tuscon, AZ & 104 & 28 & 260 & 972 & 10891 & 2.62 & 2950 & 0 & 2.58 \\
\hline Los Angeles, CA & 83 & 41 & 312 & 545 & 5575 & 2.87 & 3388 & 0 & 2.71 \\
\hline Denver, $\mathrm{CO}$ & 89 & -5 & 443 & 505 & 5403 & 2.74 & 5966 & 43 & 2.16 \\
\hline Miami, FL & 91 & 8 & 58 & 1773 & 18929 & 2.75 & 633 & 0 & 2.70 \\
\hline Atlanta, GA & 94 & 17 & 299 & 796 & 8494 & 2.75 & 3549 & 0 & 2.47 \\
\hline Hilo, HI & 84 & 61 & 28 & 1881 & 19473 & 2.83 & 302 & 0 & 2.74 \\
\hline Chicago, IL & 94 & -5 & 450 & 506 & 5377 & 2.76 & 6120 & 57 & 2.13 \\
\hline New Orleans, LA & 93 & 29 & 185 & 1170 & 12510 & 2.74 & 2074 & 0 & 2.61 \\
\hline Boston, MA & 91 & 6 & 490 & 407 & 4272 & 2.80 & 6272 & 14 & 2.28 \\
\hline Detroit, MI & 91 & 3 & 544 & 460 & 4836 & 2.79 & 7217 & 11 & 2.21 \\
\hline Saint Louis, MO & 97 & 2 & 391 & 660 & 7123 & 2.72 & 5083 & 15 & 2.25 \\
\hline New York, NY & 90 & 12 & 485 & 488 & 5087 & 2.81 & 6011 & 1 & 2.37 \\
\hline Akron, $\mathrm{OH}$ & 89 & 1 & 533 & 429 & 4483 & 2.80 & 7155 & 33 & 2.17 \\
\hline Tulsa, OK & 101 & 8 & 330 & 746 & 8176 & 2.67 & $4129^{\circ}$ & 4 & 2.34 \\
\hline Portland, OR & 89 & 17 & 485 & 237 & 2477 & 2.80 & 5651. & 0 & 2.52 \\
\hline Philadelphia, PA & 93 & 10 & 453 & 555 & 5869 & 2.77 & 5646 & 0 & 2.35 \\
\hline Providence, RI & 89 & 5 & 501 & $399^{\circ}$ & 4157 & 2.81 & 6451 & 14 & 2.27 \\
\hline Columbia, SC & 97 & 20 & 314 & 842 & 9095 & 2.71 & 3733 & 0 & 2.46 \\
\hline Nashville, TN & 97 & 9 & 338 & 723 & 7787 & 2.72 & 4202 & 10 & 2.35 \\
\hline Houston, TX & 96 & 27 & 179 & 1122 & 12067 & 2.72 & 2032 & 0 & 2.59 \\
\hline Salt Lake City, UT & 97 & 3 & 500 & 487 & 5287 & 2.70 & 6483 & 4 & 2.26 \\
\hline Seattle, WA & 84. & 21 & 629 & 195 & 2083 & 2.86 & 7311 & 0 & 2.52 \\
\hline Milwaukee, WI & $90^{\circ}$ & -8 & 507 & 400 & 4165 & 2.82 & 7088 & 93 & 2.07 \\
\hline
\end{tabular}

1. Annual energy input to heat pump, includes compressor, outdoor fan and indoor fan

2. Annual energy input to resistance heater

3. Annual cooling or heating load/annual energy input (dimensionless) 
Table 29. Annual Performance for Temperature Variation in Twenty-four Cities

Twin Air Source Heat Pump C Internal. Load of 5,000 BTUH Indoor Design Temp. of $70^{\circ} \mathrm{F}$
Cooling Design Load of 30,000 BTUH at Cooling Design Temperature Heating Design Load of 36,000 BTUH at Heating Design Temperature

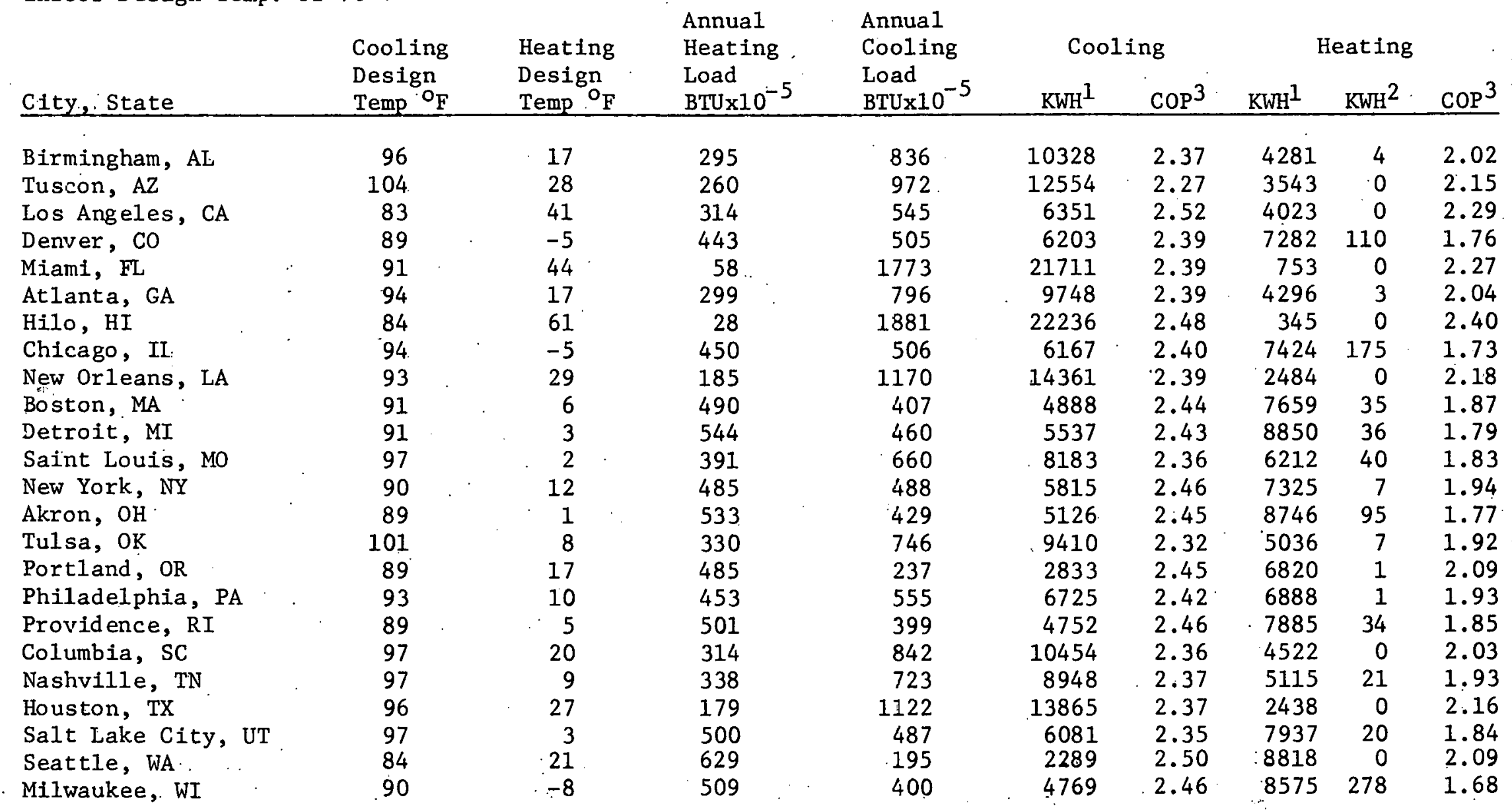

1. Annual energy input to heat pump, includes compressor, outdoor fan and indoor fan

2. Annual energy input to resistance heater

3. Annual cooling or heating load/annual energy input. (dimensionless) 
Table 30. Annual Performance for Temperature Variation in Twenty-Four Cities

Twin Air Source Heat Pump A

Internal Load of 10,000 BTUH

Indoor Design Temp. of $70^{\circ} \mathrm{F}$
Cooling Design Load of 30,000 BTUH at Cooling Design Temperature Heating Design Load of 36,000 BTUH at Heating Design Temperature

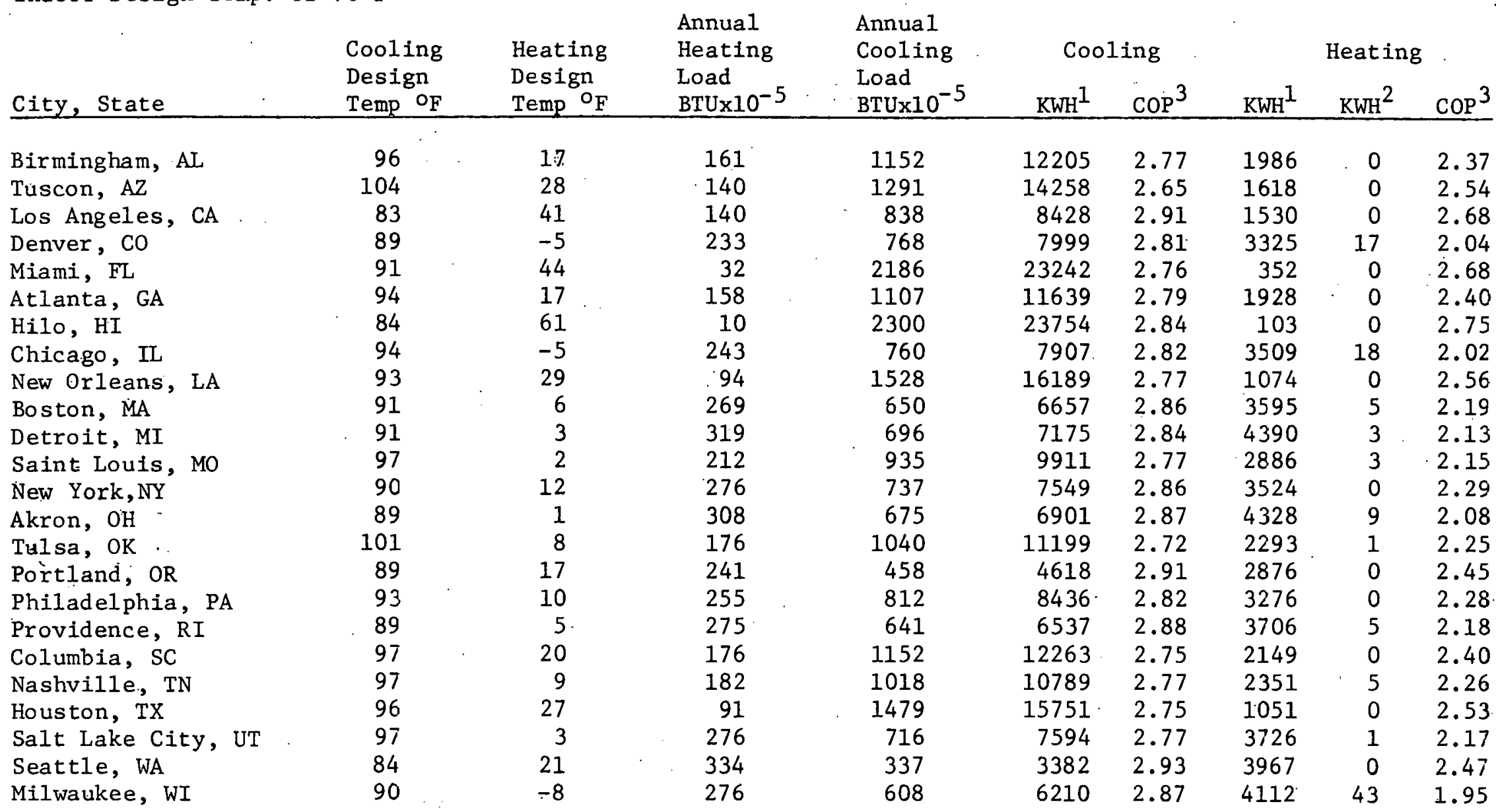

1. Annual energy input to heat pump, includes compressor, outdoor fan and indoor fan

2. Annual energy input to resistance heater

3. Annual cooling or heating load/annual energy input (dimensionless) 
Table 31. Annual Performance for Temperature Variation in Twenty-Four Cịties

Twin Air Source Heat Pump A

Internal Load of $20,000 \mathrm{BTUH}$

Indoor Design Temp. of $70^{\circ} \mathrm{F}$

\begin{tabular}{|c|c|c|c|c|c|c|c|c|c|}
\hline City, State & $\begin{array}{l}\text { Cooling } \\
\text { Design } \\
\text { Temp }{ }^{{ }^{F}}\end{array}$ & $\begin{array}{l}\text { Heating } \\
\text { Design } \\
\text { Temp }{ }^{\circ}\end{array}$ & $\begin{array}{l}\text { Annual } \\
\text { Heating } \\
\text { Load } \\
\text { BrUx } 10^{-5}\end{array}$ & $\begin{array}{l}\text { Annual } \\
\text { Cooling } \\
\text { Load } \\
\text { BTU } 10^{-5}\end{array}$ & $\mathrm{KWH}^{1}$ & $\mathrm{COP}^{3}$ & $\mathrm{KWH}^{1}$ & $\begin{array}{l}\text { Heatin } \\
\mathrm{KWH}^{2}\end{array}$ & $\mathrm{COP}^{3}$ \\
\hline Birmingham, AL & 96 & 17 & 25 & 1893 & 19676 & 2.82 & 347 & 0 & 2.14 \\
\hline Tuscon, Az & 104 & 28 & 22 & 2049 & 22136 & 2.71 & 264 & 0 & 2.39 \\
\hline Los Angeles, CA & 83 & 41 & 21 & 1594 & 15762 & 2.96 & 231 & 0 & 2.62 \\
\hline Denver, CO & 89 & -5 & 29 & 1442 & 14593 & 2.90 & 514 & 7 & 1.65 \\
\hline Miami, FL & 91 & 44 & 7 & 3038 & 32119 & 2.77 & 82 & 0 & 2.61 \\
\hline Atlanta, GA & 94 & 17 & 21 & 1848 & 19050 & 2.84 & 290 & 0 & 2.14 \\
\hline Hilo, HI & 84 & 61 & 1 & 3169 & 32590 & 2.85 & 14 & 0 & 2,78 \\
\hline Chicago, IL & 94 & -5 & 33 & 1426 & 14476 & 2.89 & 597 & 0 & 1.60 \\
\hline New Orleans, LA & 93 & 29 & 12 & 2323 & 24290 & 2.80 & 149 & 0 & 2.41 \\
\hline Boston, MA & 91 & 6 & 41 & 1299 & 12989 & 2.93 & 644 & 0 & 1.88 \\
\hline Detroit, MI & 91 & 3 & 50 & 1304 & 13139 & 2.91 & 796 & 0 & 1.83 \\
\hline Saint Louis, MO & 97 & 2 & 27 & 1628 & 16839 & 2.83 & 452 & 0 & 1.78 \\
\hline New York, NY & 90 & 12 & 42 & 1380 & 13846 & 2.92 & 605 & 0 & 2.03 \\
\hline Akron, OH & 89 & 1 & 47 & 1293 & 12941 & 2.93 & 785 & 0 & 1.76 \\
\hline Tu1sa, OK & 101 & 8 & 25 & 1765 & 18542 & 2.79 & 369 & 0 & 1.95 \\
\hline Portland, OR. & 89 & 17 & 21 & 1115 & 10941 & 2.99 & 283 & 0 & 2.20 \\
\hline Philadelphia, PA & 93 & 10 & 34 & 1468 & 14917 & 2.88 & 503 & 0 & .2 .00 \\
\hline Providence, RI & 89 & 5 & 41 & 1284 & 12789 & 2.94 & 647 & 0 & 1.87 \\
\hline Columbia, SC & 97 & 20 & 31 & 1883 & 19666 & 2.81 & 414 & 0 & 2.20 \\
\hline Nashville, TN & 97 & 9 & 24 & 1737 & 18002 & 2.83 & 368 & 0 & 1.95 \\
\hline Houston, TX & 96 & 27 & 13 & 2278 & 23925 & 2.79 & 161 & 0 & 2.35 \\
\hline Salt Lake City, UT & 97 & 3 & 36 & 1353 & 3.3895 & 2.85 & 565 & 0 & 1.85 \\
\hline Seattle, WA & 84 & 21 & 39 & 919 & 8952 & 3.01 & 515 & 0 & 2.26 \\
\hline Milwaukee, WI & 90 & -8 & $38^{\circ}$ & 1246 & 12417 & .2 .94 & 713 & 17 & 1.54 \\
\hline
\end{tabular}
Heating Design Load of 36,000 BTUH at Heating Design Temperature

1. Annual energy input to heat pump, includes compressor, outdoor fan and indoor fan

2. Annual energy input to resistance heater

3. Annual cooling or heating load/annual energy input (dimensionles's) 
THIS FROSÁS WILL ANALYZE PEFFOPMANCE AT LOCATION 1, OIRMINGHAM, ALAEAMA FOR AIR HEAT PUMP A CALCULATIONS ASE FOK INDOOR TEMPERATURE MAINTAINED AT 70. F AND INTERNAL LOAO OF SODO. BTUH

HEATING CALCULATIONS ARE FOK OUTOCOR DESIGN TEMP. OF 17. F AND LCAD OF 36000 . BTUH AT DESIGN INDOOR TEMP. OF 7O. F

COOLING CALCULFTIONS ARE FOR OUTOUOH DESIGN TEMP. OF 96. F AND LOAD OF 30000 .BTUA AT DESIGN INDOOR TEMP. OF 7O. F.

$A=$ OUTDOOR TEMPERATURE, FAHRENHEIT

$\Xi=$ SEASONAL HEATING HOURS IN 5 DEGREE INCREMENT

$C=$ SEASONAL HEATITIGICOOLING LOAD IN 5 DEGREE INCREMENT, BTU

D = HEAT PUMP CAPACITY AT. OUTDOOR TEMIPERATURE A, MBTUH

$E=$ HEAT PUMP SYSTEM INPUT, KW, INCLUDING AIF HANDLER REQUIREMENT, KW

$F=$ HEA T PUYP CONPEESSOR RUN TIME INPERCENT AT TEMPERATURE. A.

$G=S E A S O N A L$ HEAT FUMP INFUT, KWH IN $S$ DEGREE TEMPERATURE INCREMENT

$H=$ TESISTARCE HEAI KEOUIKED IN S DEGREE INCREMENT, KW

IE SEASO:AL RESISTAPCE HEAT NEEDEO IN S. DEGREE INEREMENT

$J=$ COEFICIENT OF PEKFORMANCE, DIMENSIONLESS

$K=$ SYSTEY STATUS

STANDARD CALCULATION

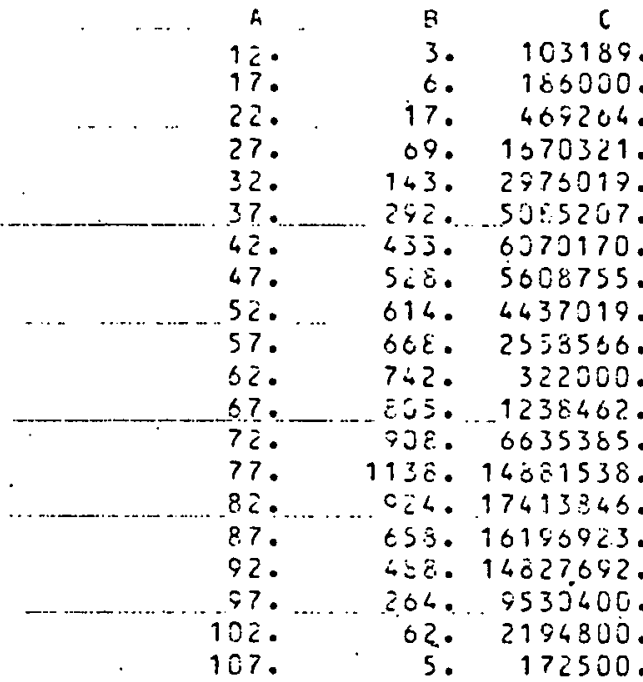

$\begin{array}{ccr}D & E & F \\ 16.9 & 3.10 & 100.0 \\ 19.2 & 3.20 & 100.0 \\ 29.7 & 3.30 & 100.0 \\ 26.4 & 3.50 & 99.2 \\ 27.1 & 3.50 & 76.8 \\ 30.1 & 3.70 & 57.9 \\ 33.0 & 3.90 & 42.5 \\ 35.9 & 4.10 & 29.6 \\ 38.5 & 4.20 & 15.8 \\ 40.8 & 4.40 & 9.4 \\ 42.7 & 4.50 & 1.0 \\ 38.3 & 3.63 & 4.0 \\ 35.1 & 3.73 & 19.2 \\ 37.9 & 3.53 & 34.5 \\ 37.6 & 3.93 & 50.1 \\ 37.2 & 4.03 & 66.2 \\ 36.7 & 4.13 & 32.8 \\ 36.1 \ldots & 4.23 \ldots & 100.0 \\ 35.4 & 4.43 & 100.0 \\ 34.5 & 4.53 & 100.0\end{array}$

$\begin{array}{rr}6 & \\ 9 . & 5.1 \\ 19 . & 3.5 \\ 56 . & 1.7 \\ 240 . & .0 \\ 395 . & .0 \\ 625 . & .0 \\ 717 . & .0 \\ 649 . & .0 \\ 484 . & .0 \\ 276 . & .0 \\ 34 . & .0 \\ 117 . & .0 \\ 650 . & .0 \\ 1504 . & .0 \\ 1820 . & .0 \\ 1755 . & .0 \\ 1369 . & .0 \\ 1117 . & .0 \\ 275 . & .0 \\ 23 . & .0\end{array}$

$\begin{array}{lr}H & 1 \\ 5.1 & 15.4 \\ 3.5 & 20.8 \\ 1.7 & 29.4 \\ .0 & .0 \\ .0 & .0 \\ .0 & .0 \\ .0 & .0 \\ .0 & .0 \\ .0 & .0 \\ .0 & .0 \\ .0 & .0 \\ .0 & .0 \\ .0 & .0 \\ .0 & .0 \\ .0 & .0 \\ .0 & .0 \\ .0 & .0 \\ .0 & .0 \\ .0 & .0 \\ .0 & .0 \\ .0 & .0\end{array}$

$J$
1.23
1.36
1.61
2.04
2.21
2.38
2.48
2.57
2.69
2.72
2.78
3.09
2.99
2.90
2.80
2.71
2.30
2.50
2.34
2.23

$k$

HEAIING

HEATING

HEATING

HEATING

HEATING

HEATING

HEATING

HEAIING

HEATING

HEATING

HEATING

COOLING

COOLING

COOLING

COOLING

COOLING

COOLING

COOLING

COOLING

ANNUAL HEATING LOAO, BTU = 29486508 .

ANSUAL HEATING HEAT PUYP INPUT, KWH =

AUVUAL RESISTINCE HEAT REQUIRED, WWH =

3496.

ANNUAL CDOLING LOSD, BTU $=83091543$.

65.

ANNUGL COOLINE MEAT PUMP INPUT, KWH

8928.

ANUUAL COOLINO COP $=.2 .73$

ADEUAL HEATING COP = 2.43

CAS HEAT LT GC\% EFFICIENCY WOULU FEQUIRE 476. THERMS.

FUEL UIL HEAT AT C $2 \%$. EFFICIENCY WOULD FEQUIRE. 328 . GALLONS 
HEATING CALCULATIONS ARE FOH OUTDOOR DESIGN TEMP. OF 28 . F AND LOAD OF 36000 . BTUH AT DESIGN INDOOR TEMP. OF 70. F

COOLIIVG CALCULATIONS ARE FOR OUTDOOR DESIGN TEMP. OF1OL. F AND LOAD OF 3OOOO.BTUH AT DESIGN INDOOR TEMP. OF 7O. F.

$A=$ OUTDODF TEMPERATURE.,FAHRENHEIT

$B=$ SEASONAL HEATING HOURS IN 5 OFGREE INCREMENT

C... SEASONAL...HEATING/COOLING. LOAD..IN. S. DEGREE... INCREMENT.., BTU

D = HEAT PUYP CAPACITY AT OUTDOOR TEMPERATURE A, MIETUH

E = HEAT PUMP SYSTEM INPUT, KW, IMCLUDING AIR HANDLER REQUIREMENT, KW

FF = HEAT...PUMP CONIPKESSOR. RUN TIME. INPERCENT AT TEMPERATURE A

$G=$ SEASONAL HEAT PUMP INPUT, KWH IN $S$ DEGREE TEMPERATURE INCREMENT

$H=$ RESISTANCE HEAT REQUIREO IN 5 DEGREE INCKEMENT, KW

I. SEASONAL RESISTANCE. HEAT. NEEDED. IN. S. DEGKEE. INCREMENT.

$J=$ COEFICIENT OF PERFORMANCE, DIMENSIONLESS

$K=$ SYSTEM STATUS

STANDARD CALCULATION

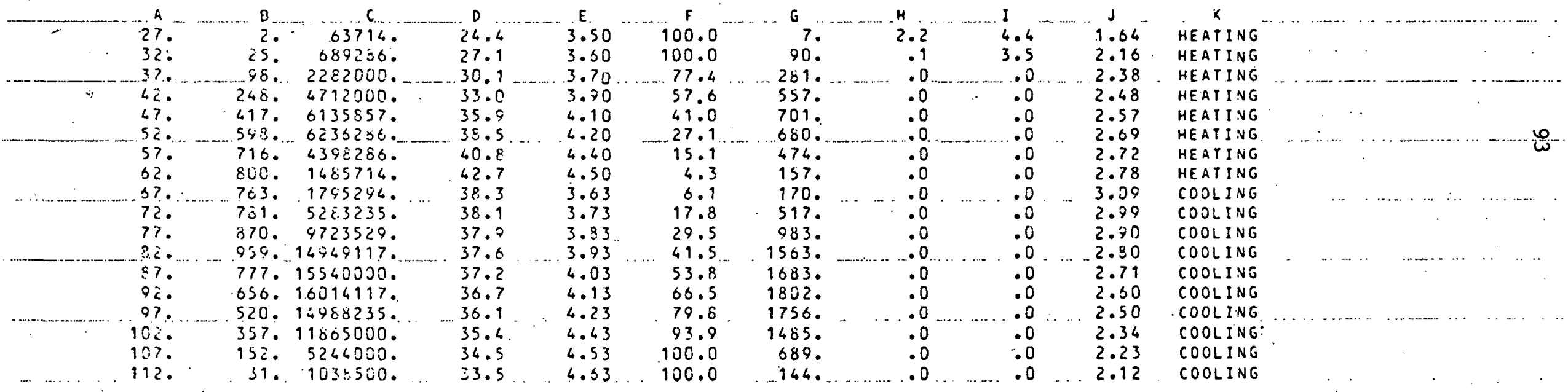

ANNUAL HEATING LOAD, BTU $=20003142$.

ANVUAL TEATING HEAT PUMP INPUT, KWH =

ANYUAL RESISTANCE HEAT R.EOUIRED, KNH =

ANNUAL COOLING LOAD, ETU $=96449026$. 2946.

ANPUAL COOLING. HEAT PUMP INPUT, KWH $=\ldots . . .10791$.

ANNUAL COOLING COP $=2.62$

ANNUAL HEATJNG COP = 2.58

GAS HEAT AT. 62\% EFFICIENCY WOULO REQUIRE
FUEL CIL HEAT AT O2\% EFFICIENCY WOULD REQUIRE
289. GALLONS 


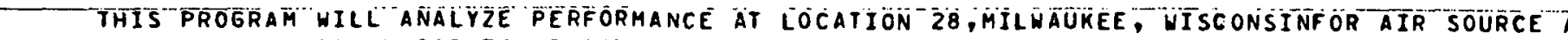

CALCULATIONS ARE FOR INOOOR TEMPERATURE MAINTAINED AT TO. F AND INTERNAL LOAD OF SODO. BTUH

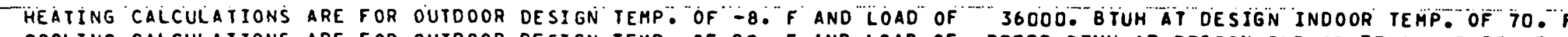

COOLING CALCULATIONS ARE FOR OUTDOOR DESIGN TEMP. OF 90. F AND LOAD OF 3OOOD.BIUH AT OESIGN INDOOR TEMP. OF 7O. F.

AE OUTDOOR TEMPERATURE, FAHRE NHEIT

$B=$ SEASONAL HEATING HOURS IN 5 OEGREE INCREMENT

$C=$ SEASONAL HEATING/COOLING LOAD IN 5 DEGREE INCREMENT, BTU

D = HEAT PUMP CAPACITY AT OUTOOOR TEMPERATURE A, MBTUH

$E=$ HEAT PUMP SYSTEM INPUT, KW, INCLUDING AIR HANDLER REQUIREMENT, KH

$F=$ HEAT PUMP COMPRESSOR RUN TIHE INPERCENT AT TEMPERATURE A

G:- SEASONAL HEAT PUMP INPUT, KHH IN 5 DEGREE IEMPERATURE INCREMENT

$H=$ RESISTANCE HEAT REQUIREO IN 5 OEGREE INCREMENT, $K H$

I = SEASONAL RESISTANCE HEAT NEEDED IN 5 DEGREE INCREMENT

$J=$ COEFICIENT OF PERFORMANCE, DIMENSIONLESS

$K=$ SYSTEM STATUS

STANDARO CALCULATION

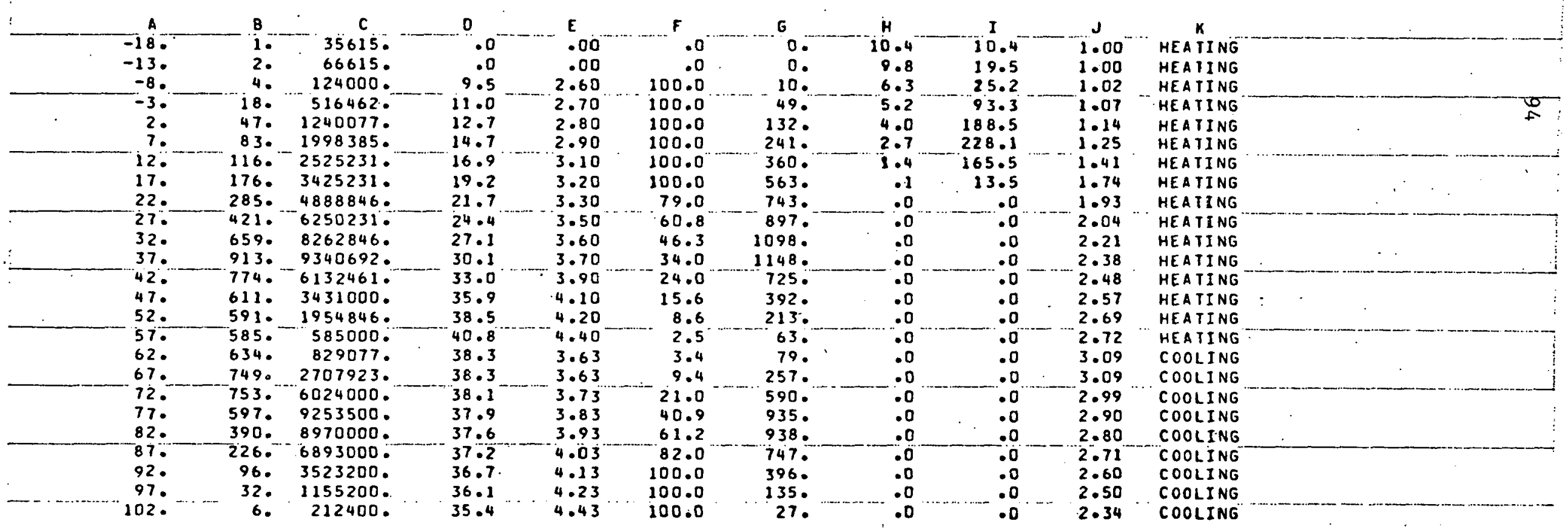

ANNUAL HEATING LOAD, BTU $=50777535$.

ANNUAL HEATING HEAT PUMP INPUT, KHH =

ANNUAL RESISTANCE HEAT REOUIRED, KWH =

ANNUAL COOLING LOAD, BTU $=39568299$.

ANNUAL COOLING HEAT PUMP INPUT, KWH"

ANNUAL COOLING COP $=2.83$
ANNUAL HEATING COP $=2.02$

ANNUAL HEATING COP $=2.02$

FUEL C HEAT AT $62 \%$ EFFICIENCY WOULD REQUIRE

6633.

744.

4103

819." "THERMS.

565. GALLONS 
HEATING CALCULATIONS ARE FOR OUTDOOR DESIGN TEMP. OF -8. AND LOAD OF 36000 . BTUH AT DESIGN INDOOR TEMP. OF TO. F COOLING CALCULATIONS ARE FOR OUTOOOR OESIGN PEMP. OF 9O. F AND LOAD OF 3OOOO.BTUH AT DESIGN INDOOR TEMP. OF TO. F.

A = OUTTOÖOR TEMPERATURE, FAHRENHEIT

$B=$ SEASONAL HEAIING HOURS IN 5 OEGREE INCREMENT

-C C SEASONAL HEATINGICOOLING LOAD IN S DEGREE. INCREMENT , BTU

$D=$ HEAT PUMP. CAPACITY AT OUTDOOR -TEMPERATURE A, MBTUH

$E=$ HEAT PUMP SYSTEM INPUT, KH, INCLUOING AIR HANDLER REQUIREMENT, $K H$

- F= HEAT PUMP COMPRESSOR RUN TIME INPERCENT AT TEMPERATURE A

$G=$ SEASONAL HEAT PUMP INPUT, KWH IN 5 DEGREE TEMPERATURE INCREMENT

$H=$ RESISTANCE HEAT REQUIRED IN 5 DEGREE INCREMENT, KH

- I ... SEASONAL RESISTANCE HEAT NEEDED IN 5 DEGREE INCREMENT

$J=$ COEFICIENT OF PERFORMANCE. DIMENSIONLESS

$K=$ SYSTEM STATUS

STANDARD CALCULATION

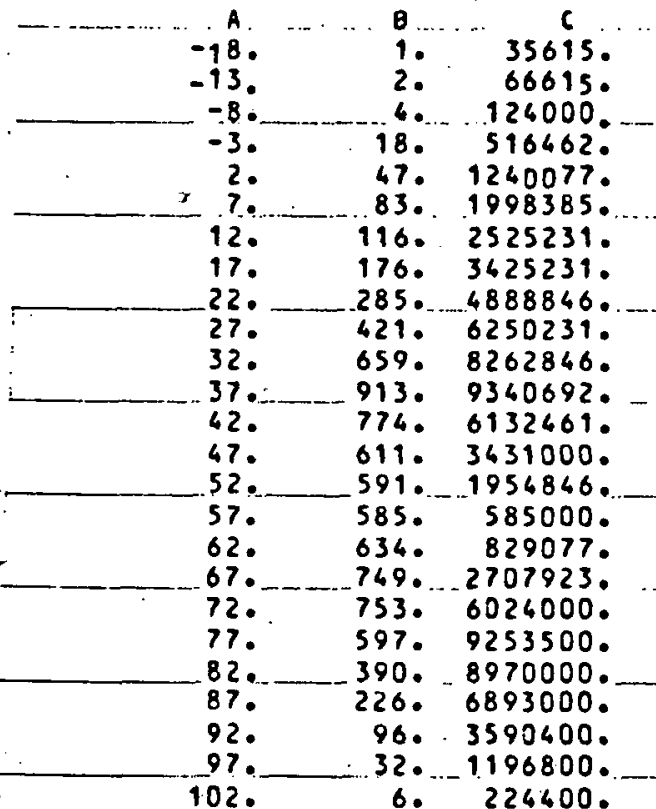

0
52.0
52.0
52.0
52.0
52.0
52.0
52.0
52.0
52.0
52.0
52.0
52.0
52.0
52.0
52.0
52.0
37.4
37.6
37.6
37.6
37.4
37.6
37.4
37.4
37.6

$E$
5.62
5.62
5.62
5.62
5.62
5.52
5.62
5.62
5.62
5.62
5.62
5.62
5.62
5.62
5.62
5.62
4.66
4.66
4.66
4.66
4.66
4.66
4.66
4.66
4.66

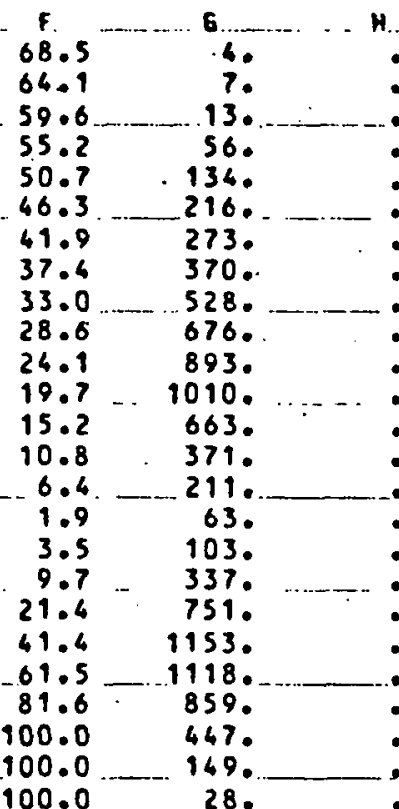

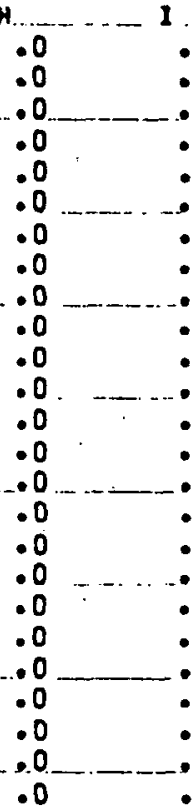

$\begin{array}{ll}1.0 & \\ 1.0 & 2.71 \\ .0 & 2.71 \\ .0 & 2.71 \\ .0 & 2.71 \\ .0 & 2.71 \\ .0 & 2.71 \\ .0 & 2.71 \\ .0 & 2.71 \\ .0 & 2.71 \\ .0 & 2.79 \\ .0 & 2.79 \\ .0 & 2.71 \\ .0 & 2.71 \\ .0 & 2.71 \\ .0 & 2.71 \\ .0 & 2.71 \\ .0 & 2.35 \\ .0 & 2.35 \\ .0 & 2.35 \\ .0 & 2.35 \\ .0 & 2.35 \\ .0 & -2.35 \\ .0 & 2.35 \\ .0 & 2.35 \\ .0 & 2.35 \\ .0 & 2.35\end{array}$

MEATIMG

HEATING

HEATING

HEATING.

HEATING

HEATING

HEATING

HEAIING

HEATING

HEATING

HEATING

HEATING

HEATING

HEATING

HEATING

HEATING

COOLING

COOLING

COOLING

COOLING

COOLING

COOLING

COOLING

COOLIRG

ANKUAL HEATING LOAD, BTU = S0777535

ANNUAL HEATING HEAT PUMP INPUT, KHA =

ANNUAL RESISTANCE HEAT REQUIRED, KHH =

ANNUAL COOLJNG LOAD, BTU = 39689100 .

ANNUAL COOLING HEAT PUMP INPUT, KWH

ANNUAL COOLING COP $=2.35$

ANNUAL HEATING COP $=2.71$

GAS HEAT AT 62X EFFICIENCY WOULD REQUIRE 819 . THERMS.

FUEL OIL HEAT AT 62X EFFICIENCY WOULD REQUIRE 565. GALLONS 
THIS PROSRAM HILL ANATYYZE" PERFORMANCE AT LOCATION 28, MILWAUKEE, WISCONSINFOR TUIN AIR A CALCULATIONS ARE FOR INDOOR TEMPERATURE MAINTAINEO AT 70. F AND INTERNAL LOAD OF 5OOO. BTUH

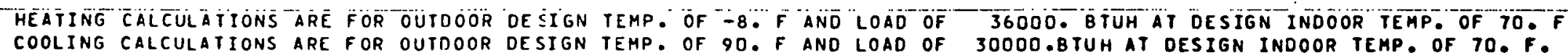

Ä OUTDOOR TEMPERATURE, FAHRENHEIT

$B=$ SEASONAL HEATING HOURS IN 5 OEGREE INCREMENT

CE SEASONAL HEATING/COOLING LOAO IN 5 DEGREE - INCREMENT... BTU

DE HEAT PUMP CAPACITY AT OUTOOOR TEMPERATURE A, MBTUH

E = HEAT PUMP SYSTEM INPUT, KH, INCLUDING AIR HANDLER REQUIREMENT, KH

$F=$ HEAT PUMP COMPRESSOR RUN TIME INPERCENT AT TEMPERATURE A

$G=-$ SEASONAL HEAT PUHP INPUT; KUH IN S DEGREE TEMPERATURE INCRËHENT

H= RESISTANCE HEAT REOUIRED IN 5 DEGREE INCREMEN.T, KW

I = SEASONAL RESISTANCE HEAT NEEOED IN 5 DEGREE INCREMENT

$J=$ COEFICIENT OF PERFORMANCE, DIMENSIONLESS

$K=$ SYSTEM STATUS

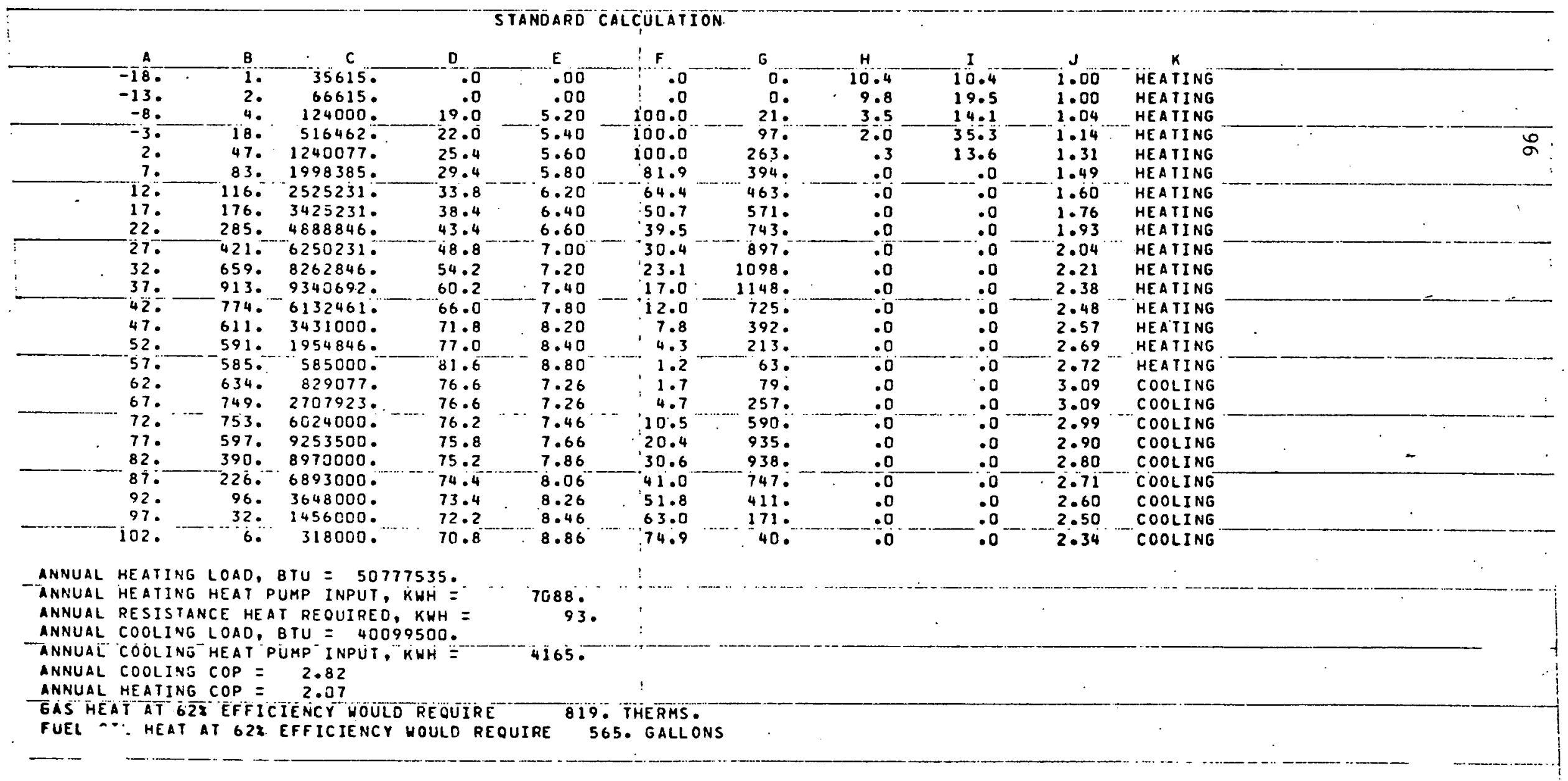


THIS PROGRAH HILL ANALYZE PERFORMANCE AT LOCATION 28, MILHAUKEE, HISCONSINFOR THIN AIR A CALCULATIONS ARE FOR INDOOR TEMPERATURE MAINTAINED AT 70. F AND INTERNAL LOAO OF 1OODO. BTUH

HEATING CALCULATITONS ARE FOR OUTTOOOR DESIGN TEMP. OF-8. F AND LOAD OF 360OO. BTUH AT DESIGN INDOOR TEMP. OF TO. F

COOLING CALCULATIONS ARE FOR OUTOOOR DESIGN TEMP. OF 90. F AND LOAD OF 3OOOO.BTUH AT DESIGN INDOOR TEMP. OF 70. F.

A $=$ OUTOOOR TEMPERATURE, FAHRENHEIT

$B=$ SEASONAL HEATING HOURS IN 5 DEEREE INCREMENT

$C=$ SEASONAL HEATING/COOLING LOAD IN 5 DEGREE INCREMENT, BTU

DE HEAT PUMP CAPACITY AT OUTDOOR TEAPERATURE A, MBTUH

E = HEAT PUMP SYSTEM INPUT, KH, INCLUDING AIR HANOLER REOUIREMENT, KH

FE HEAT PUMP COMPRESSOR RUN TIME INPERCENT AT TEMPERATURE A

$G=$ SEASONAL HEAT PUMP INPUT, KWH IN 5 DEGREE TEMPERATURE INCREMENT

$H=$ RESISTANCE HEAT REQUIRED IN 5 DEGREE INCREMENT, KH

$I=$ SEASONAL RESISTANCE HEAT NEEDED IN 5 DEGREE INCREMENI

$J=$ COEFICIENT OF PERFORMANCE, OIMENSIONLESS

$K=$ SYSTEM STATUS

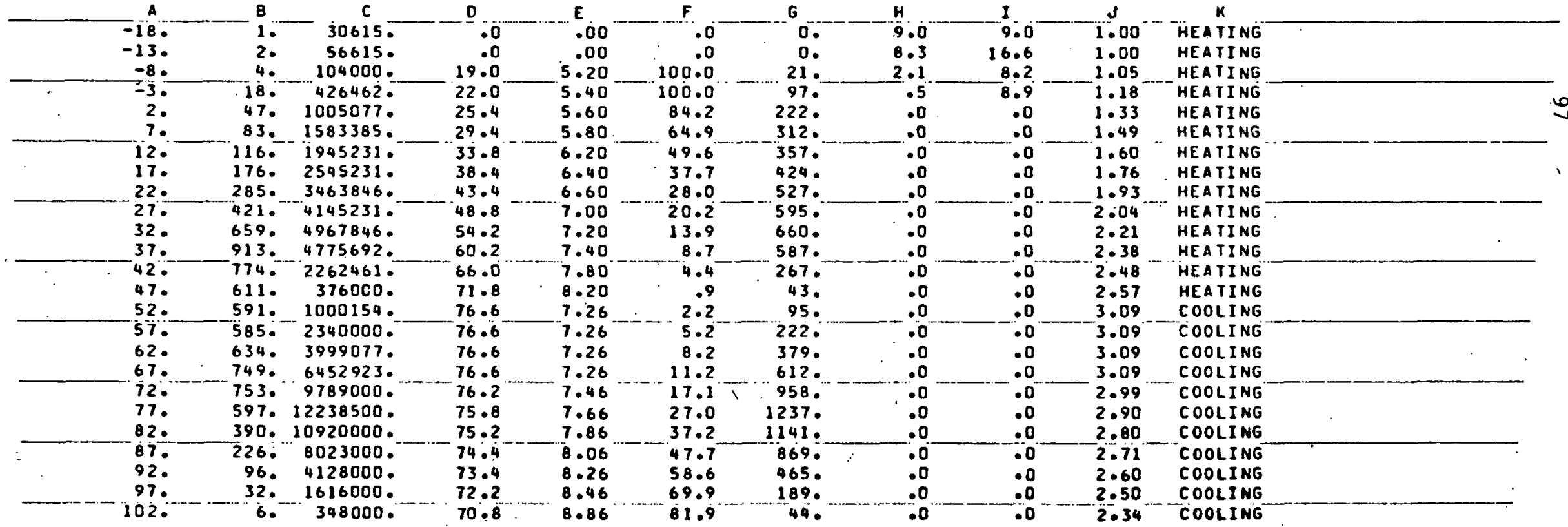

ANNUAL HEATING LOAD, BTU $=27687691$

ANNUAL HEATING HEAT PUMP INPUT, KWH =

ANNUAL RESISTANCE HEAT REQUIRED, KHH =

ANNUAL COOLING LOAD, BTU $=60854653$.

ANNUAL COOLING HEAT PUMP INPUT, KUH

4112.

ANNUAL COOLING COP $=2.87$

ANNUAL HEATING COP $=1.95$

GAS HEAT AT 62\% EFFICIENCY WOULO REOUIRE - 447 . THERMS.

FUEL OIL HEAT AT $62 \%$ EFFICIENCY WOULO REOUIRE 308. GALLONS 
HEATING CALCULATIONS ARE FOR OUTDOOR DESIGN TEMP. OF -8. F AND LOAO OF 36OOO. BTUH AT OESIGN INDOOR TEHP. OF TO. F COOLING CALCULATIONS ARE FOR OUTOOOR DESIGN TEMP. OF'90. F AND LOAO OF 3ODOO.8TUH AT DESIGN INDOOR TEMP. OF 70. F.

AE OUTDOOR TEMPERATURE, F̈AKRENHETT

$B=$ SEASONAL HEATING HOURS IN 5 DEGREE INCREMENT

$C=$ SEASONAL HEATING/COOLING LOAD IN S DEGREE INCREMENT .. BIU

D = HEAT PUMP CAPACITY AT OUTOOOR TEMPERATURE A, MBTUH

E = HEAT PUMP SYSTEM INPUT, KH, INCLUDING AIR HANDLER REQUIREMENT, KH

F= HEAT. PUMP COMPRESSOR RUN TIME INPERCENT AT TEMPERATURE A

6 SEASONAL HEAT PUMP INPUT, KHH IN 5 DEGREE TEMPERATURE INCREMENT

H= RESISTANCE HEAT REOUIRED IN 5 DEGREE INCREMENT, KH

I = SEASONAL RESISTANCE HEAT NEEDED IN 5 DEGREE INCREMENT

$J=$ COEFICIENT OF PERFORMANCE. DIMENSIONLESS

$K=$ SYSTEM STATUS

STANOARO CALCQULATIOON

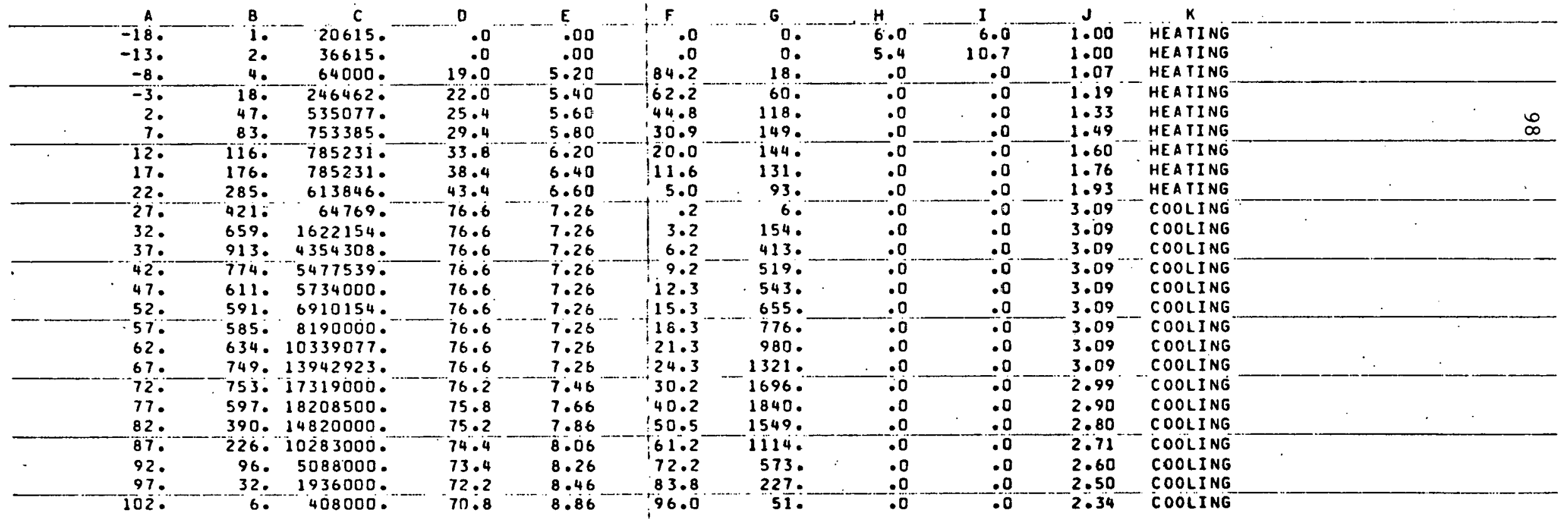

ANNUAL HEAIING LOAD, BTU $=3840461$.

ANNUAL HEATING HEAT PUMP INPUT, KHH

ANNUAL RESISTANCE HEAT REQUIRED, KHH =

ANNUAL COOLING LOAO, 8 TU $=124697423$

ANNUAL COOLING HEAT PUMP INPUT, KHH

ANNUAL COOLING COP $=2.94$

ANNUAL HEATING COP $=1.54$

GAS HEATA AT 62 E EFFICIENCY WOULD REQUTRE

713.

62. THERMS".

FUEL OIL HEAT AT 628 EFFICIENCY NOULD REOUIRE 43. GALLONS 
heat pumps and air source heat pumps is in packaging and controls. The water source heat pump is available with both heat exchangers, compressor, and air handler in one packaged unit. Physically the water heat exchanger is smaller than the air source heat exchanger. The air source unit requires one external and one internal package. Also, due to external exposure the air source units require compressor crankcase heating and heat exchanger fin defrost. The controls to operate the system must be included. As a result the installed cost of the air source units is appreciably more than that of the water-source unit. The installed cost per Christian is listed in the pump performance Table 1 and Tables 23 to 25 . A surcharge of $\$ 300$ has been added to the high performance air source heat pump A, and a $\$ 300$ surcharge has been added to the modified revisions of water source heat pumps with the additional heat exchanger. This charge is similar to a duct $A$ frame heat exchanger for a 3-ton heat pump. It must be noted that fixed prices for heat pumps do not exist as they vary from region to region and dealer to dealer.

According to Christian, the prices for water source pumps compared to air source heat pumps on the basis of ARI capacity are one-half as much. ${ }^{10,11}$ However, the water-source heat pump provides essentially a constant heat output, a function of water temperature; whereas the air-source unit shows a rapid decline in heating output at low ambient temperatures. Comparisons of costs at low ambient temperatures may disclose a price ratio of as much as $4: 8$. As a result, this heating deficit must be eliminated by resistance heating.

Exception is taken to Christian's estimate of maintenance cost. 10,11 
In as much as the water source heat pump is a much simpler system and operates over a smaller temperature range, less maintenance and service should be required. Due to the smaller temperature range resulting in lower compressor pressure ratios, a comparison with refrigerator maintenance and depreciation is possible. Of 150 units installed in the Tuscaloosa VA Hospital, only three service calls were required the first year. All three calls were for burned-out fan motors.

\section{IX.B. Aquifer Analysis}

The movement, occurrence, and distribution of water below the surface is known as groundwater hydrology. The groundwater referred to without further specification is commonly understood to mean water occupying all the voids within a geological stratum or a portion thereof. The saturated zone is to be distinguished from an unsaturated, or aeration, zone where voids are filled with water and air. This study is confined to the saturated zones for use as energy sinks and sources. However, the unsaturated zones may contain soil moisture and interact with the saturated zones, especially at shal-low depths. The study is also limited to one-phase flow, water only. Combination of water and gas or water and oil are not considered.

Groundwater plays a critical role in the United States with over $50,000,000,000$ gallons per day being required for public supplies, rural use, irrigation, and industrial supplies. As a result, the hydrology of groundwater is a well-defined science. The storage of thermal energy is basically undefined on a scientific basis. Numerous experiments or attempts at storage on small scales were carried out in the late $40^{\prime} \mathrm{s}$ and $50^{\prime} \mathrm{s}$ in conjunction with system development for heating and cooling.

The basic problems with the systems were unreliable equipment and poorly 
engineered storage systems. One of the prime problems was using a finite volume as an infinite heat sink and source. General studies have shown the earth to be an extremely poor conductor of thermal energy.

The average temperatures of shallow groundwater as a function of 1ocation in the United States are shown in Figure $21 .^{19}$ These are temperatures of water near the surface; temperature normally increases with depth on a uniform basis. Temperature of water in all cases noted on the map is suitable for heating purposes; however, use of water below $50^{\circ} \mathrm{F}$ is questionable. To improve heat pump performance, this-project proposes to employ water with an initial temperature of $45^{\circ} \mathrm{F}$ or higher and to use return water that has been injected at temperatures as high as $100^{\circ} \mathrm{F}$. Much of the cold water, below $55^{\circ} \mathrm{F}$, can bc used directly for cooling without the utilization of a heat pump. However, large scale use may lower the water table and reduce the availability of water.

The flow of water in an aquifer is a function of Darcy's 1aw. ${ }^{17,18}$ Basically the flow rate can be given as:

$$
\mathrm{Q}=\mathrm{KA} \frac{\mathrm{dh}}{\mathrm{dL}}
$$

where $Q$ is the flow rate,

$\frac{\mathrm{dh}}{\mathrm{dL}}$ is the hydraulic gradient

$A$ is the cross-sectional area, and

$K$ is the constant of proportionality known as the coefficient of permeability

The equation is sometimes written with a negative sign to show flow in the opposite direction from the gradient of the hydraulic head. The equation's validity is limited to laminar flow. Essentially all aquifer flow, except for underground streams or the area immediately adjacent to the 


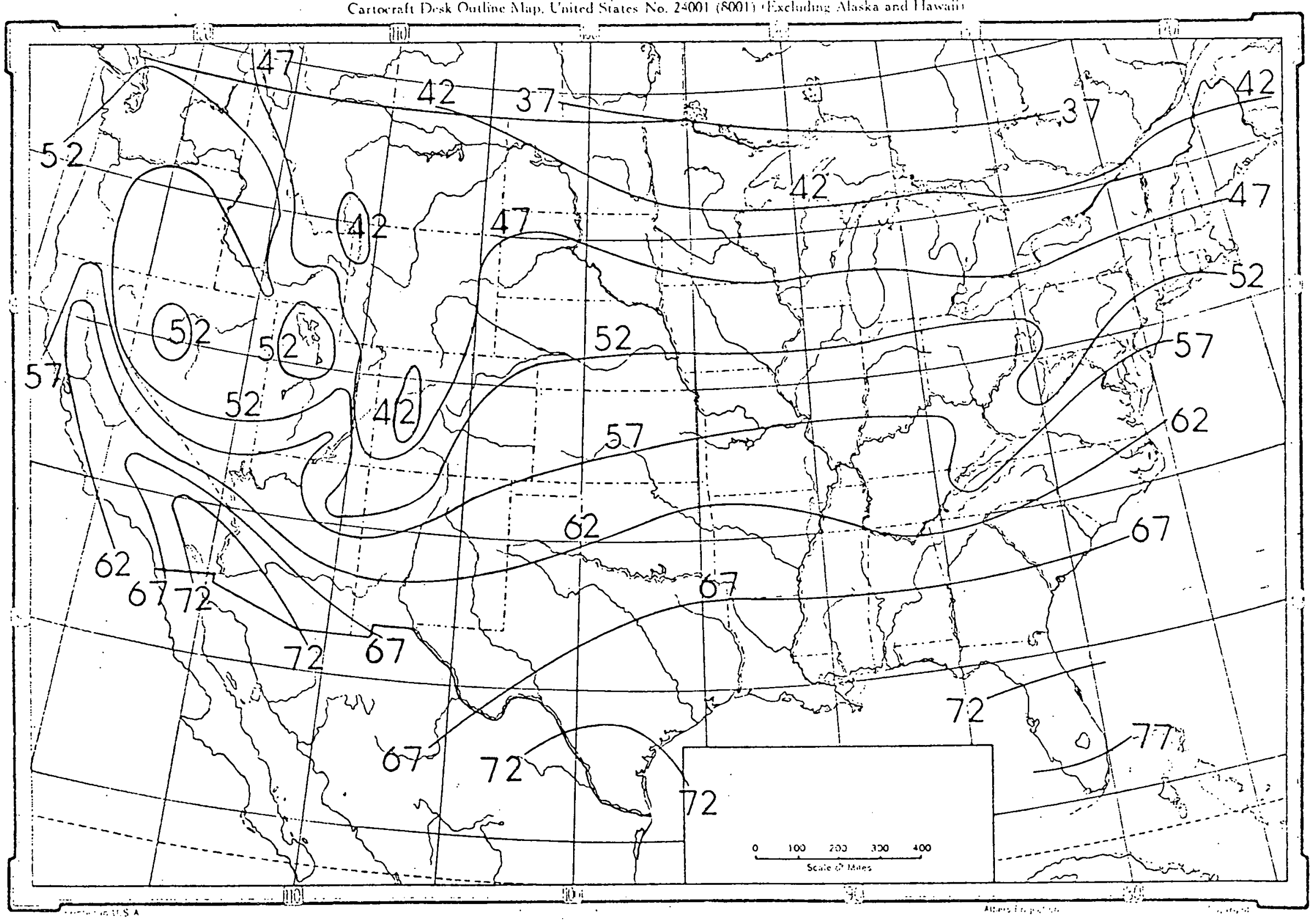

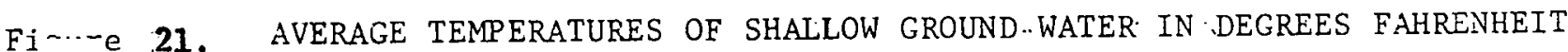


well bore, is laminar flow.

The laboratory coefficient of permeability $K_{s}$ is defined as a flow of 1 gal./day through a cross-sectional area of $1 \mathrm{ft} .{ }^{2}$ with a hydraulic gradient of $1 \mathrm{ft.} / \mathrm{ft}$. and at a temperature of $60^{\circ} \mathrm{F}$. A specific permeability "k" known as the darcy may also be used by separating out the properties of the fluid. A darcy is expressed as:

$$
k=\frac{\mu Q}{\gamma A(d h / d L)}
$$

where $\mu$ is fluid viscosity, and

$\gamma$ is fluid specific weight

The relationship between the darcy and the laboratory coefficient of permeability is:

$$
1 \mathrm{darcy}=18.2 \mathrm{~K}_{\mathrm{s}}
$$

The values of permeability for different types of aquifers are shown in Figure 22.17

The rate of flow of ground water is governed by permeability of the aquifer and the hydraulic gradient. Normal flow rates range between 5 $\mathrm{ft./year} \mathrm{to} 5 \mathrm{ft./day.} \mathrm{Most} \mathrm{aquifers} \mathrm{considered} \mathrm{for} \mathrm{thermal} \mathrm{storage} \mathrm{will}$ have flow rates of less than $100 \mathrm{ft} . /$ year.

As water is removed by a well from an aquifer, the velocity increases from essentially zero at a relatively long distance from the we11, to maximum velocity at the well. Analyzing the flow capacity as a function of distance from a well with radius $r$ using Darcy's law,

$$
Q=A K \frac{d h}{d r}=2 \pi r b K \frac{d h}{d r}
$$

where $b$ is thickness of the water-bearing portion of we11. ${ }^{17}$ Rearranging and integrating for boundary conditions of $r=r_{w}$ and $h=h_{w}$ for conditions at the well and $r=r_{0}$ and $h=h_{0}$ at a distance from the well yields: 


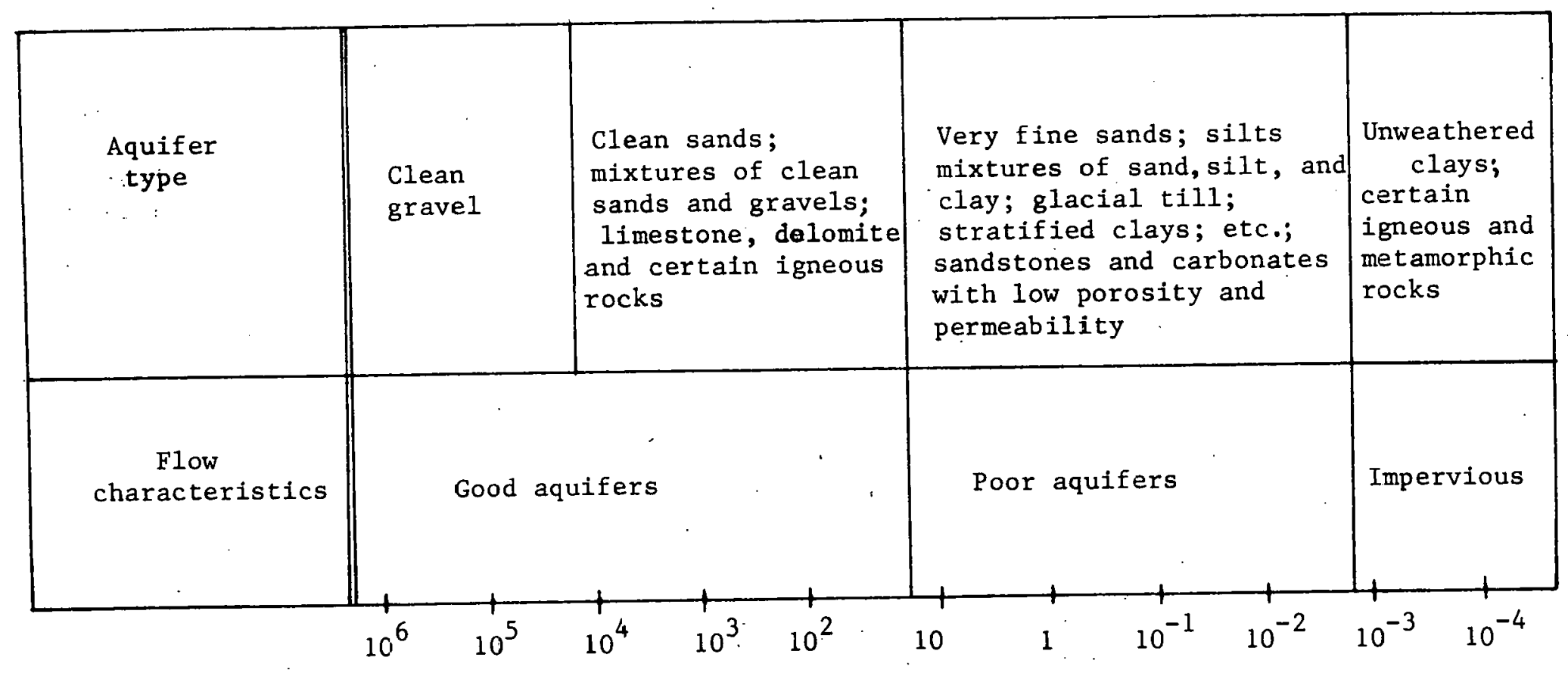

Figure 22 LABORATOB* CORRFICIRNT OF PERMEABILATY; $\mathrm{K}_{\mathrm{g}}$ GAL./DAY $\mathrm{ft} .{ }^{2}$ AT A HYDRAULIC GRADIENT OF $1 \mathrm{ft} . / \mathrm{ft}$. (Modified after Todd, 1959). 


$$
Q=2 \pi K b \frac{h_{o}-h_{w}}{\ln \left(r_{o} / r_{w}\right)}
$$

The head " $h$ " varies linearly with the logarithm of the distance $r$ for all flow rates. Figure 4, included earlier, shows a schematic of the fluctuations in $h$ for a confined aquifer for a system with injection and withdrawal wells. As a result of this relationship, a good approximation of the head loss between a withdrawal and an injection well is twice the sum of the head differential from a well to a point halfway between the wells.: Figure 23 shows the pressure as related to head loss between a pair of wells based on these relationships for three values of permeability. The values are aquifer-permeability ranges in the United States.

Aquifers of adequate permeability are prerequisite to the use of water-source heat pump systems. As an example, a pair of 6 ranch diameter wells with a flow of $200 \mathrm{ga} 1 . / \mathrm{min} .\left(2.88 \times\left(10^{5}\right) \mathrm{gal} / \mathrm{day}\right)$ between the wells spaced $500 \mathrm{ft}$. apart in an aquifer $100 \mathrm{ft}$. thick would produce a head differential of approximately:

$$
\begin{aligned}
& 0.04 \mathrm{ft} \text {. at a permeability of } 25,000 \mathrm{gal} . / \text { day } \mathrm{ft} .2 \\
& 0.09 \mathrm{ft} \text {. at a permeability of } 10,000 \mathrm{gal} \text {./day } \mathrm{ft} .2 \\
& 0.90 \mathrm{ft} \text {. at a permeability of } 1,000 \mathrm{gal} . / \text { day } \mathrm{ft} .2 \\
& 9.00 \mathrm{ft} \text {. at a permeability of } 100 \mathrm{gal} . / \text { day } \mathrm{ft} .2 \\
& 90.00 \mathrm{ft} \text {. at a permeability of } 10 \mathrm{gal} . / \text { day } \mathrm{ft} .2 \\
& 900.00 \mathrm{ft} \text {. at a permeability of } 1 \mathrm{gal} \text {. day } \mathrm{ft} .{ }^{2}
\end{aligned}
$$

Based upon the above calculations, a source that would serve the system design proposed in this study is limited to aquifers with permeabilities somewhat greater than 1 gal./day-ft. ${ }^{2}$. A minimum of more than $30 \mathrm{gal} . / \mathrm{day}-\mathrm{ft} .{ }^{2}$ would be more realistic.

The flow patterns in the aquifers, resulting from low velocities, i.e. less than $1 \mathrm{ft} . / \mathrm{sec}$, are essentially potential flow except in the immediate vicinity of a stream or well. This has been verified by laboratory 


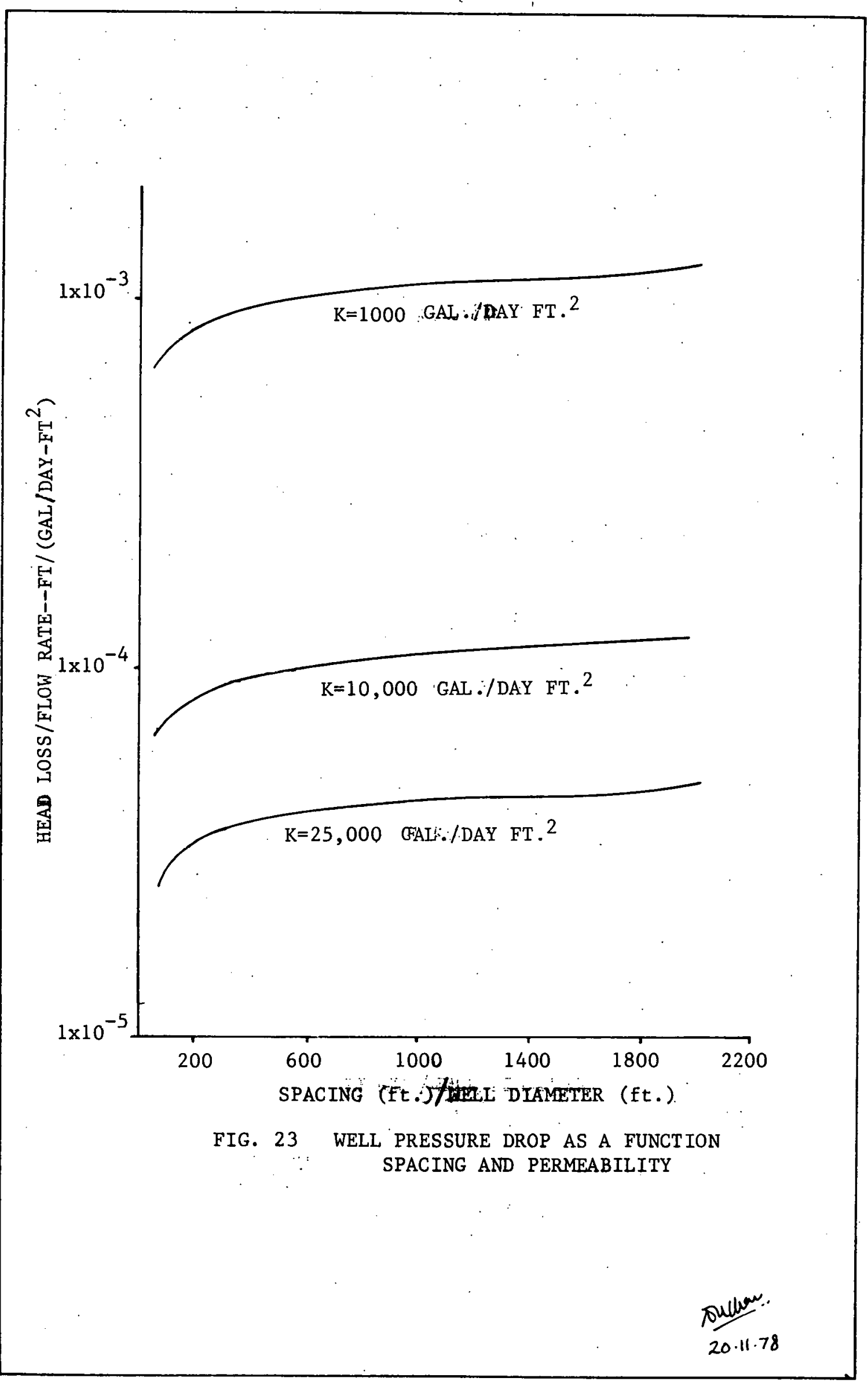


and field tests., 2122 patterns for a pair of wells, one injection and one withdrawal, are shown in Figures 24 to 27 . These flow patterns have been developed assuming potential flow and matching constant stream function and potential function values. 23,29 the curves show visually the patterns of ground water velocities under various conditions. Figure 24 . includes only the flow from the wells. Figures 25 to 27 have imposed ground flows. The ground-flow velocity has been arbitrarily chosen so its magnitude is equal to one-half the pre-existing velocity. The flow patterns indicate that either well could be used as the injection or with-: drawal well. Upon withdrawal of water, however, a mixing of aquifer water and injected water will occur after a period of time. The result will be a decrease in system performance of a few percent. In turn, the mixed water will provide a buffer zone where the supply water is not completely cold or warm. This effect will be more noticeable with the second and third alternative systems where some direct heating and cooling occur with the water.

A potential flow field with equal sources and sinks (withdrawal wells and injection wells) has the velocity profile shown in Figure 29. By integrating the velocity (function of flow rate) with respect to time along the center lines between the two wells, a relationship between distance, time, and flow rate can be established. Figure 28 gives the time required for the water to traverse between the 2 wells where flow rate is a function of flow rate per unit thickness of the aquifer.

Since only a portion of the volume (porous part of the aquifer) is water-bearing, the actual velocity, based on the concept of continuity, is 


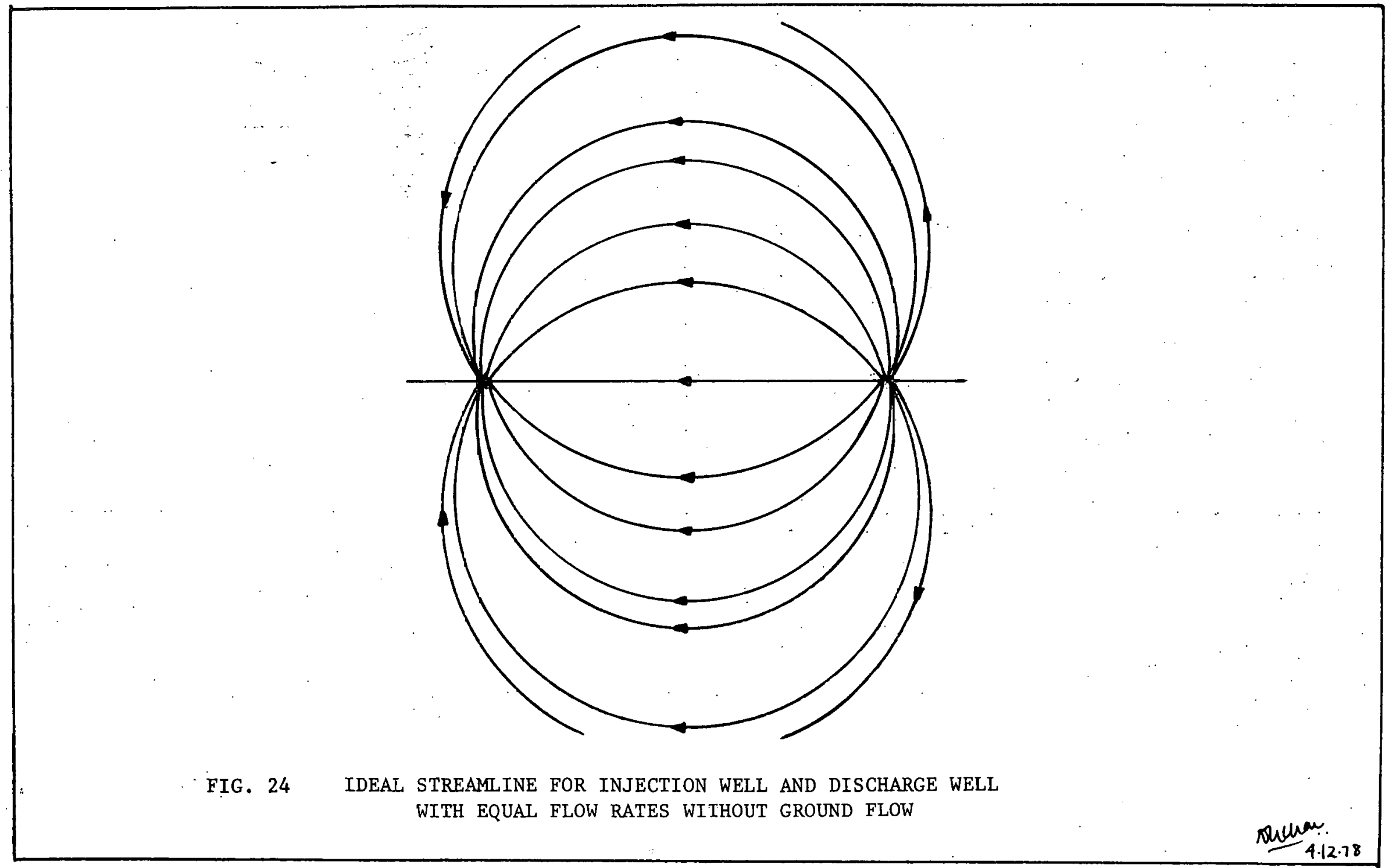




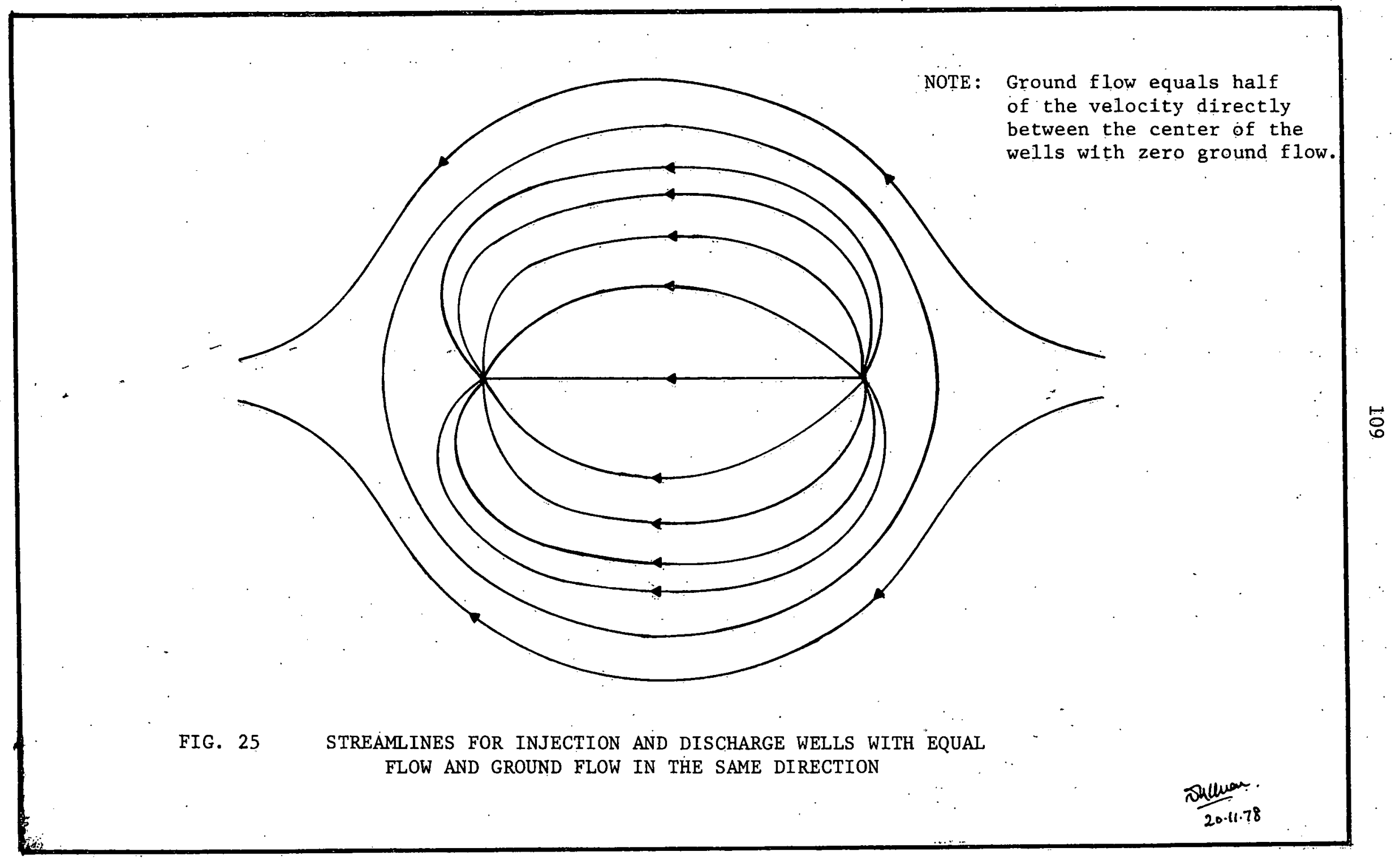




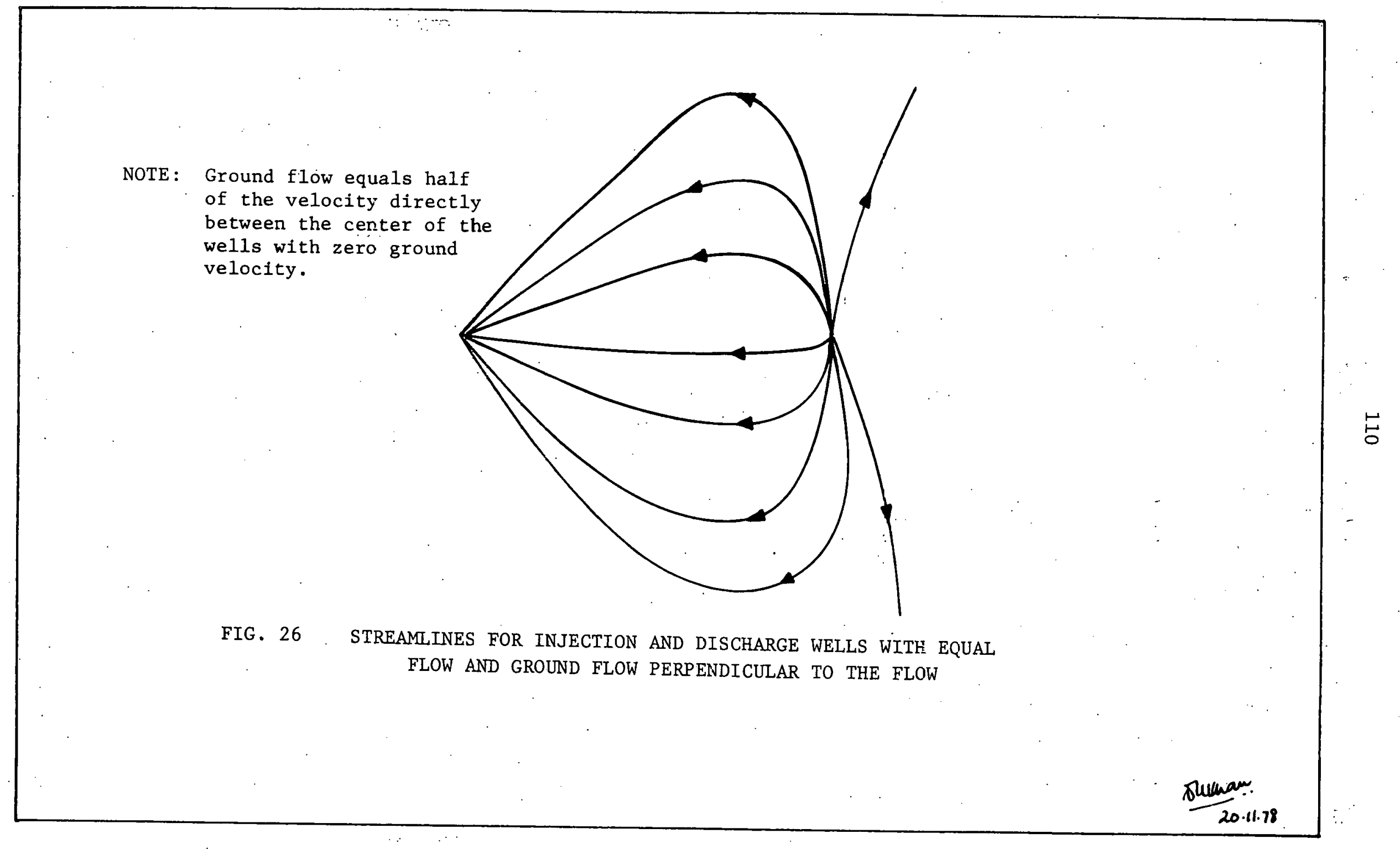




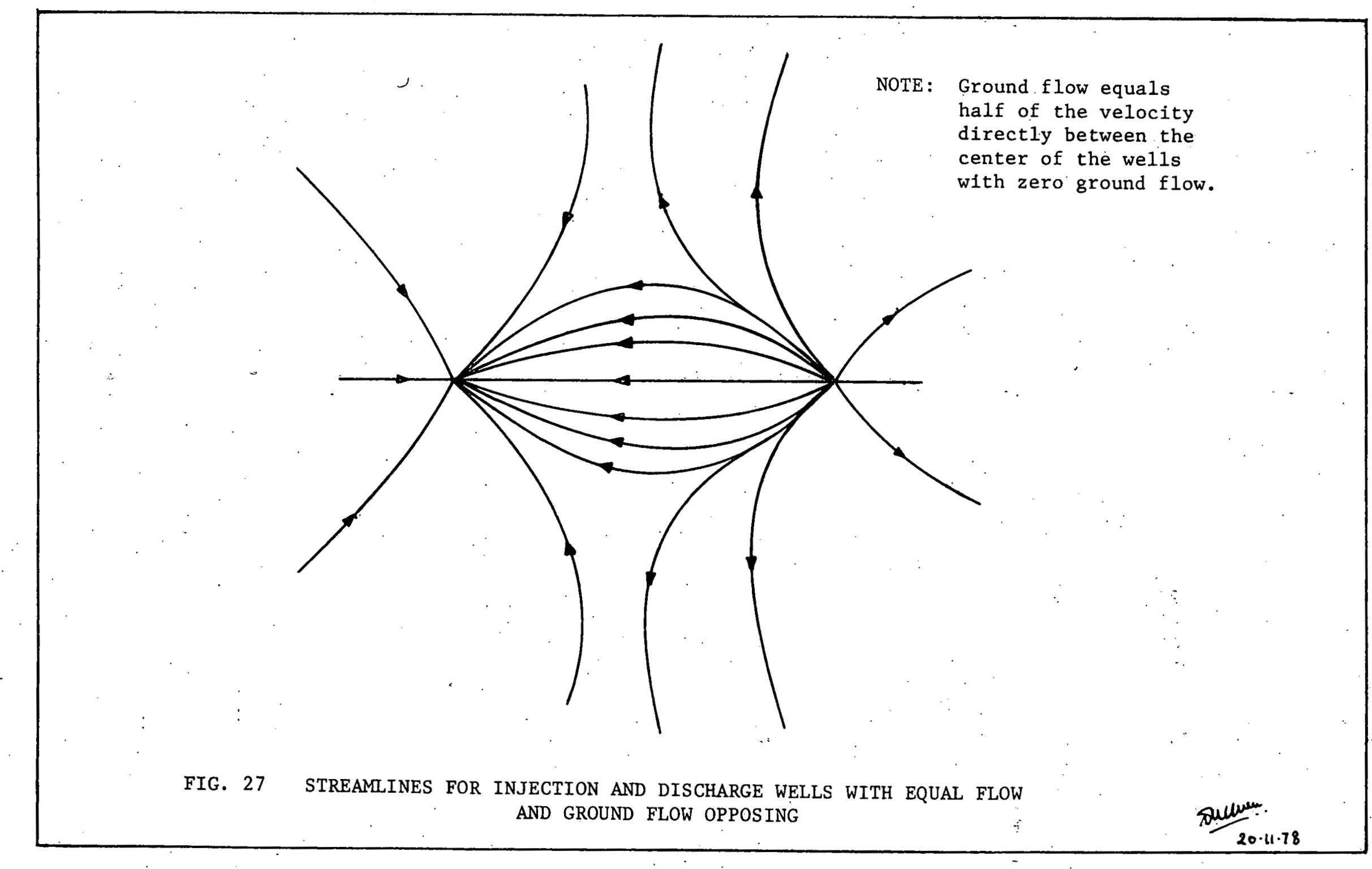




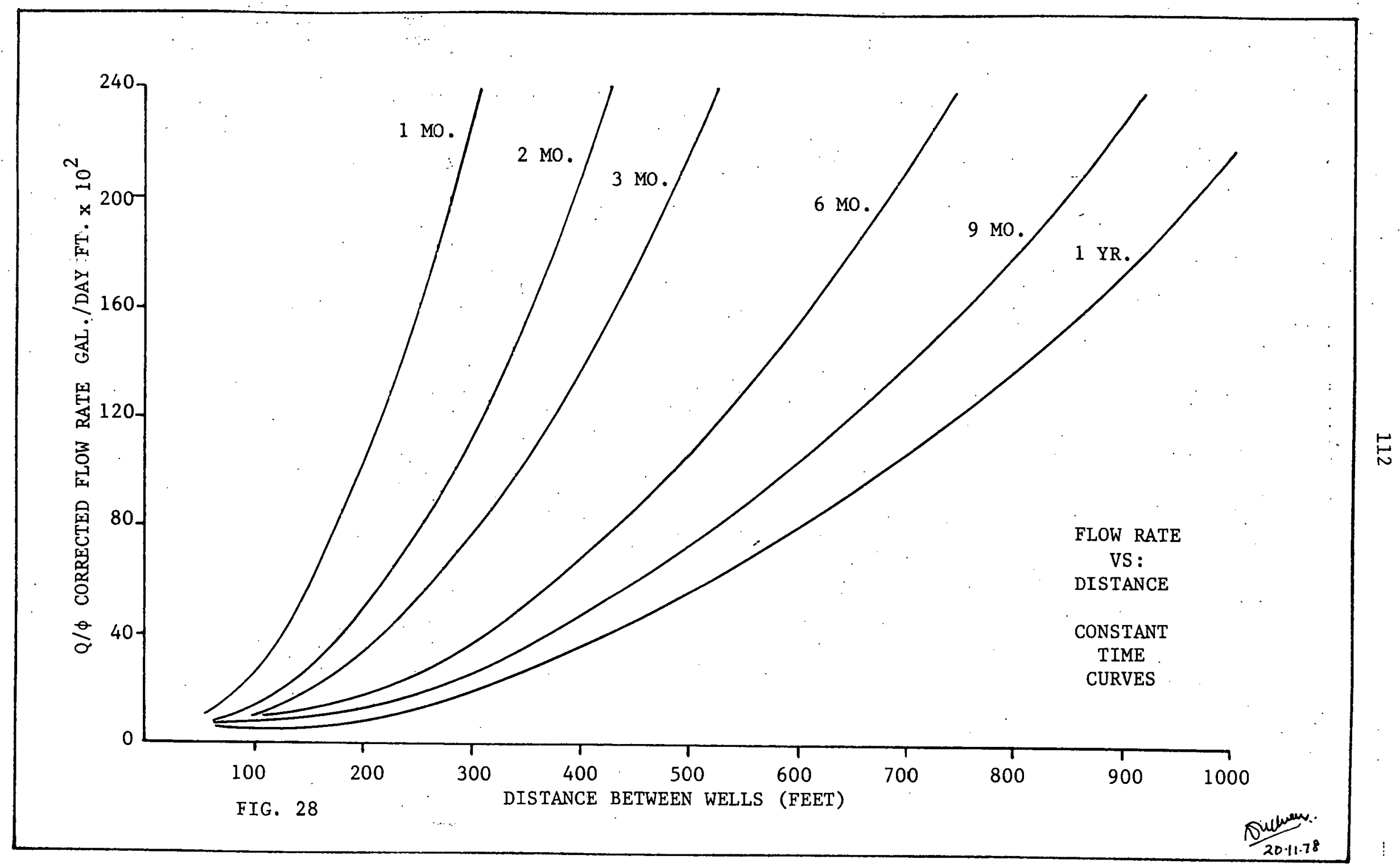


appreciably greater. Velocity will increase by the ratio of void per unit of cross-sectional area. It is assumed that this ratio relates to porosity as defined on a volume basis rather than an area basis. To determine the flow rate in an aquifer, the actual flow rate must be divided by the porosity. This means a flow rate of $100 \mathrm{gal}$./day per ft. of aquifer thickness with $10 \%$ porosity requires the same time to traverse between 2 wells as 1,000 gal./day per ft. though $100 \%$ porosity, or unrestricted flow.

For the previous example of $200 \mathrm{gal} / \mathrm{min} .\left(2.88\left(10^{5}\right) \mathrm{gal} . / \mathrm{day}\right)$ between 2 wells $500 \mathrm{ft}$, apart in a $100 \mathrm{ft}$.-thick aquifer, the corrected flow rate and the time for water to move between the wells are:

$\begin{array}{lllr}\text { Corrected Flow Rate } & \text { Traverse Time } & \text { Porosity } \\ \left(10^{3}\right) \text { gal./day-ft. } & \text { Over 1 year } & 100 \% \\ 5.76\left(10^{3}\right) \text { gal./day-ft. } & \text { Over 1 year } & 50 \% \\ 2.88\left(10^{4}\right) \text { gal./day-ft. } & \text { About 3 months } & 10 \%\end{array}$

For the proposed system, the time for the water front to pass between the 2 wells is unimportant, but the time for the temperature front to pass between the 2 wells is critical.

To determine the velocity of the temperature front, 2 assumptions are made. First, the water temperature and rock temperature at any location in the aquifer are assumed to be identical. This is valid if the contact area between the water and rock is very large for the amount of heat transfer between the masses; which condition is true where the flow channels are numerous and very small, such as the passages for water in a bucket of sand. Second, the thermal conductivity through aquifers is very small or basically negligible. This is supported by the fact that relatively small temperature changes take place underground: Measurements at The University of Alabama have detected less than a $1 / 2^{\circ} \mathrm{F}$ temperature change at $8 \mathrm{ft}$. 
below the surface over an extended period of time. In the proposed systems well spacing is expected to be in the range of a few hundred feet (100-1,000 ft. ), further supporting frontal-movement assumptions utilized herein.

Based on these assumptions, a temperature front follows the injected water front through the aquifer as a step function. This also means the temperature-wave process is reversed when the water is withdrawn. The result is that the energy stored in the aquifer is retrieved at the same temperature level as when injected. Or, stated otherwise-- $80^{\circ} \mathrm{F}$ water injected into a $50^{\circ} \mathrm{F}$ aquifer will theoretically be recovered at $80^{\circ} \mathrm{F}$. The situation in practice will be very close to the theoretical assumption. The phenomenon is demonstrated physically every day in the home. When the hot water valve of a mixing spigot is turned on, hot water begins to move through the pipe. As the water moves through the pipe, the pipe acquires the same temperature as the water. Once the water begins to be warm at the spigot, it is hot almost instantly. This demonstrates injection. The recovery is demonstrated as the cold water valve is opened. The water remains warm while the pipe is warm but turns cold almost instantly with the flow of cold water. The temperature function moves as a step function at a velocity lower than water velocity. The aquifer process will be much closer to the theoretical case. The time required for the water to change temperature at the spigot always seems excessively long. This occurs because the temperature wave moves slower than the velocity wave or at a relatively low velocity compared to the water front. In an aquifer, the temperature-front velocity is proportional to the ratio of the fluid thermal capacity divided by fluid-plus-rock thermal capacity times the water front velocity or: 


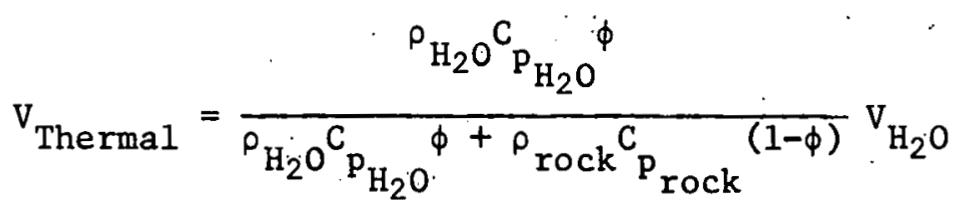

where $\rho$ equals density

$C_{p}$ equals specific heat, and

$\phi$ is aquifer porosity

To determine the time required for the temperature wave to move between wells, the time for the water must be multiplied by the reciprocal of the same thermal capacity ratio as the velocities. The times, given in Figure 28, change as:

$$
t_{\text {Thermal }}=\frac{\rho_{\mathrm{H}_{2} \mathrm{O}} \mathrm{C}_{\mathrm{p}_{\mathrm{H}_{2} \mathrm{O}}} \phi_{\rho_{\text {rock }}}+\rho_{\mathrm{p}_{\text {rock }}}(1-\phi)}{\rho_{\mathrm{H}_{2} \mathrm{O}} \mathrm{C}_{\mathrm{P}_{\mathrm{H}_{2} \mathrm{O}} \phi}} t_{\text {Water }}
$$

The time for the temperature front to move through the aquifer is the critical time, not the time of water-front movement. Well spacing and water flow rates must be considered on the basis of thermal times.

The ratio of $t_{\text {Thermal }}$ to $t$ Velocity equals
10.88 for a porosity of 0.05
5.68 for a porosity of 0.10
3.99 for a porosity of 0.15

or the temperature frontal-movement time for $200 \mathrm{gal} / \mathrm{min}$. in an aquifer $100 \mathrm{ft}$. In thickness with $10 \%$ porosity is 5.68 times 3 months, or about 17 months, as indicated in the early calculations. This gives a "safety factor" of approximately 3 in determining well spacing. A $100 \mathrm{gal} . / \mathrm{min}$. well in an aquifer $50 \mathrm{ft}$. thick with $10 \%$ porosity would result in the same thermal travel time. In all the states, information is available with respect to ground water availability. Data collected by state geological surveys, the U. S. Geological Survey, and other water-resources organizations include aquifer depth, nature, thickness, and potential yield. Water-quality 
analyses are available in many instances. In Alabama many of the wells drilled, both private and public, are logged, tested for water flow, and analyzed for mineral content in the water. Figure 29 is a map of Alabama depicting 5 formations suited for supply wells in the proposed system. Also shown are the several wells which have been logged and tested. It may be noted the formations are identified by local geographic names. These equivalent formations are referred to by different names in different states. Figure 30 shows the extent of these formations in a portion of the United States.

IX.C. Wells

The we11 is the conduit between the aquifer and the surface. Well construction is governed by a number of factors such as depth, type of soil and rock penetrated, aquifer characteristics, expected yield, and cost. These variables also relate to the method of drilling employed, the diameter of the hole, and the casing program.

Two broad categories of well types are generally recognized in the United States--first, wells which penetrate relatively "soft" soil and rock and are completed in water-bearing alluvium or sand and gravel above bedrock and second, wells which penetrate are completed in indurated rock such as sandstone, limestone, or dolomite. In certain instances, metamorphic and igneous rocks containing, fractures or other voids may serve as suitable aquifers.

Three basic types of drilling equipment are employed, percussion or cable-tool rigs, augers, and rotary tools. The choice of equipment is governed primarily by the factors noted above. Of these, cost is a major consideration. 


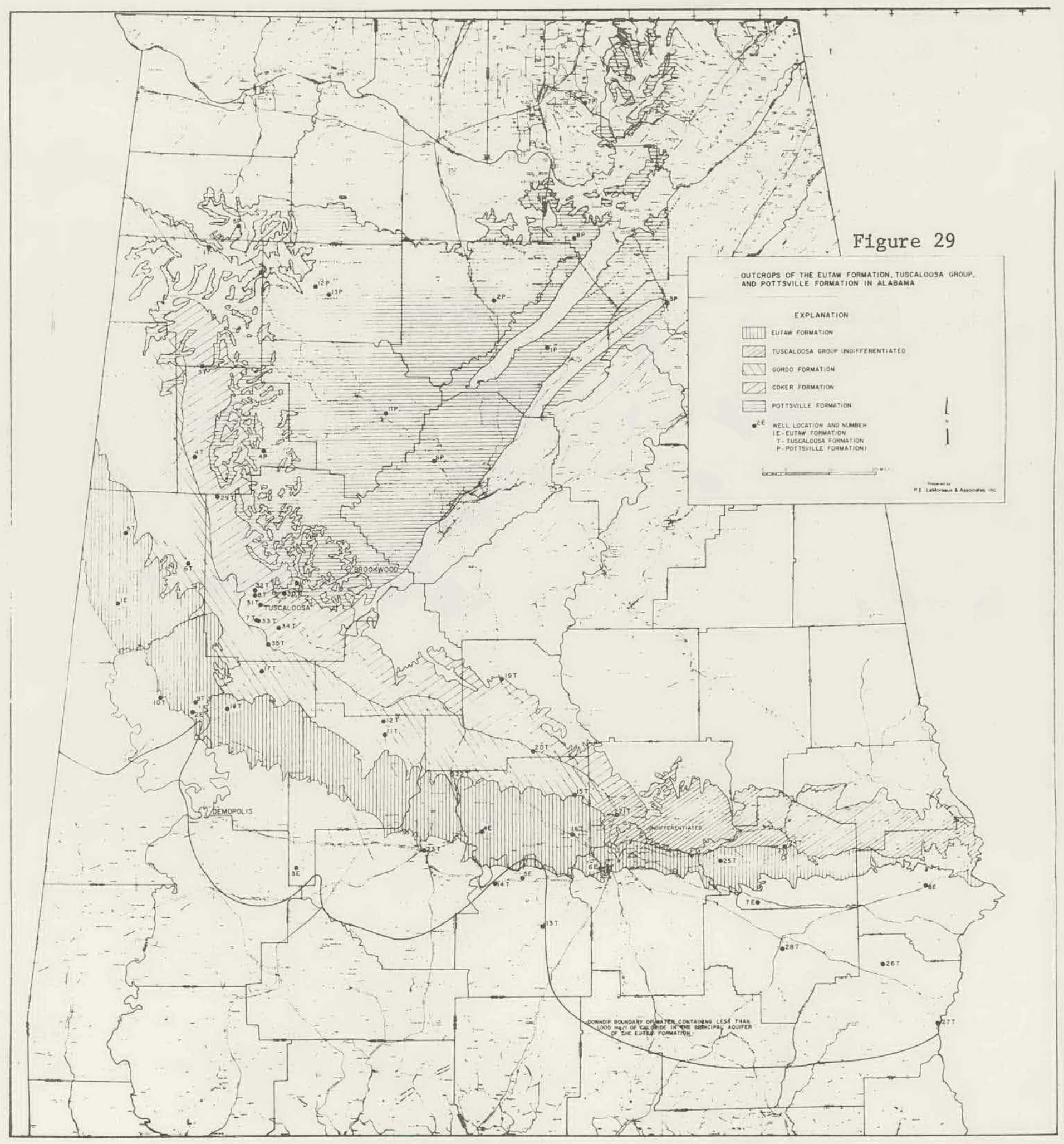




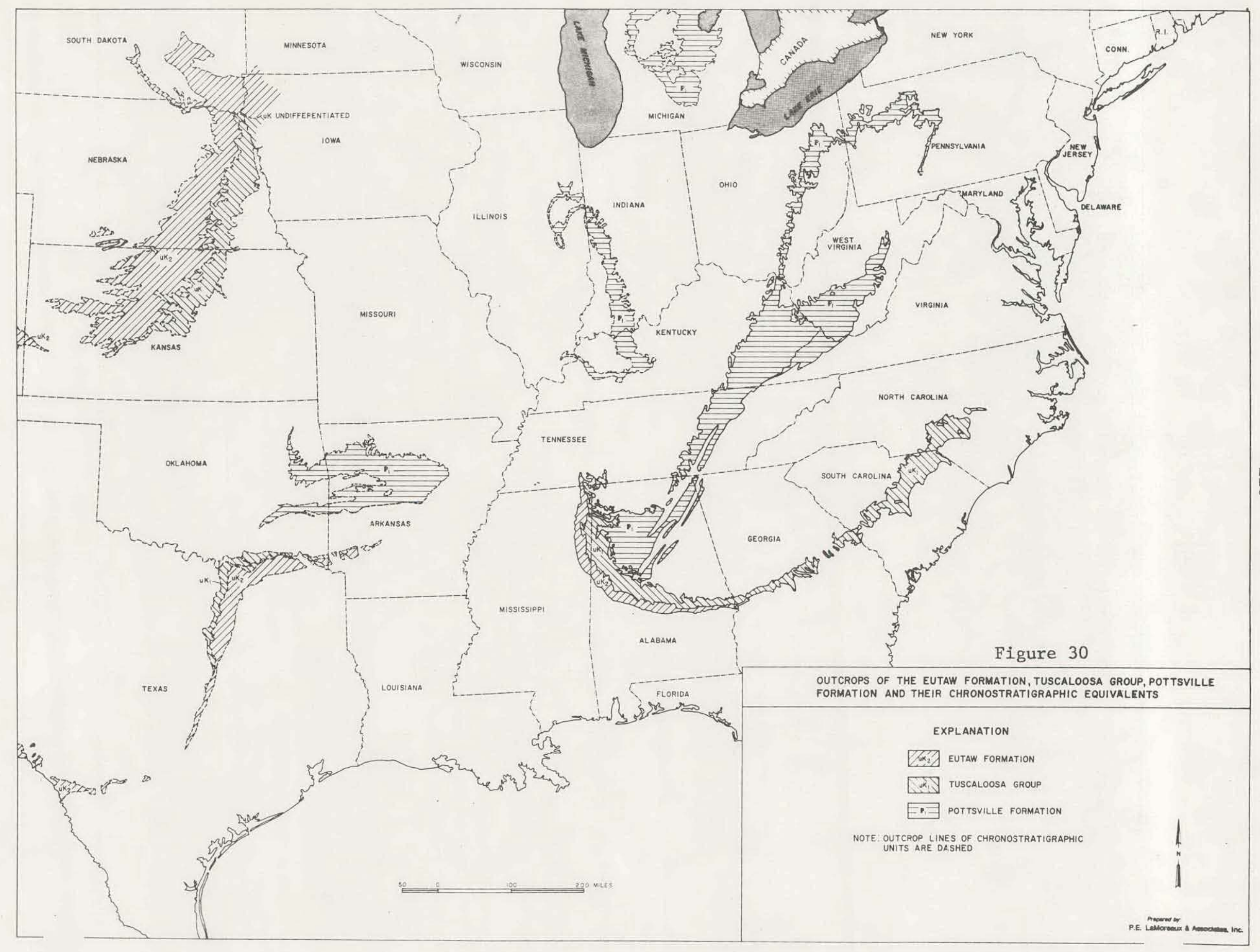


The cost of construction of water wells in the United States falls within a rather broad range for wells of a given depth, related primarily to the type of geologic formation penetrated. Table 39 gives average price estimates as of 1978 for construction of wells in the depth categories of 100 and $200 \mathrm{ft}$. Wells within this depth range and diameters shown in the table will generally provide adequate water for domestic or light industrial use, although there is no means other than experience and intimate knowledge of aquifers in an area to predict the yield of a particular well. Other things being equal, well diameter, a controllable factor, is one determinant of the volume of flow from an aquifer into the well.

In considering cost estimates for the construction of wells, provision should be made for contingencies that may be of considerable significance. As an example, a particular project may have to absorb the cost of test holes or unsuccessful wells. Enlarging the diameter of a well by reaming may be required to increase yield. Other unforseen problems may arise that add materially to the cost of a supply system.

The ideal aquifer for this system might be one. in the depth range of 100 to $150 \mathrm{ft}$. encountering 50 or more $\mathrm{ft}$. of porous and highly permeable water-bearing section. In the study area, an example would be sands and gravels within the Tuscaloosa group; the areal distribution of these formations is shown in Figure 29. Wells of 6-inch diameter constructed in aquifers of this type should be expected to yield in excess of $100 \mathrm{GPM}$, adequate to serve a 20-home module.

The well system used for this study consists of a 6-inch diameter well with an assumed water-head depth of $30 \mathrm{ft}$. Lowering of head in the withdrawal well by $20 \mathrm{ft}$. raises the head in the injection well by 20 ft., assuming the aquifer properties are constant and fiow rates are 
Table 39. Average Water-We11 Drilling Costs in the United States, 1978(a)

Casing diameter, Aquifer inches

4
6
6
24
6
6

type (b)

Sand and Grave1

Sand and Grave1

Sand and Gravel

Sand and Grave1.

Sandstone and Limestone

Sandstone and Limestone

\begin{tabular}{|c|c|c|}
\hline $\begin{array}{l}\text { Drilling } \\
\operatorname{Method}(\mathrm{c})\end{array}$ & $\begin{array}{l}\text { Depth, } \\
\text { Feet }\end{array}$ & $\begin{array}{l}\text { Cost Range } \\
\text { Dollars (d) }\end{array}$ \\
\hline Cable tools & 200 & $1500-1750$ \\
\hline Cable tools & 200 & $2400-2800$ \\
\hline Rotary & 100 & $1500-1750$ \\
\hline Auger: & 100 & $1200-1575$ \\
\hline Rotary & 100 & $900-1200$ \\
\hline Rotary & 200 & $1550-1900$ \\
\hline
\end{tabular}

(a) Personal communication, Mr. Michael Everly, National Water Well Association, Worthington, Ohio 43085. Additional references: Gibb, J.P., Cost of Domestic Wells As Water Treatment In Illinois, Circular 104, Illinois State Water Survey, Urbans, 1971; and Cederstrom, D.J.., Cost Analysis Of Ground-Water Supplies In The North Atlantic Region, U.S. Geological Survey Water-Supply Paper 2034, 1973.

(b) Refers to (1) generally unconsolidated sand, gravel and siltstones occurring above bedrock as opposed to (2) indurated sandstones, limestones and dolomites, or "bedrock".

(c) The percussion or cable-tool method of drilling, used extensively in early years, is still applicable in areas where geological formations are competent. Rotary drilling may employ either air or hydraulic systems for removal of drill cuttings.

(d) Costs based on 1970 data adjusted to 1978 economic level, including installed curbing or casing and screens as required.

In many cases, a considerable part of drilling costs involves the expense of moving in, setting up, transporting supplies (principally water), and moving out. The proposed system plans to locate wells along right-of-ways or other accessible places, to space wells no more than 1,000 feet apart, and to enter. into multiple-well contracts, thus minimizing moving and related costs. In addition, rock types will be known, which will serve to reduce contingencies that add to contract prices. 
equal. The ideal work required for primary pumping at a flow rate of 5 GPM, 50-ft. head in the well, 10-ft. head in pipelines (well below values for $100 \mathrm{GPM}$ shown in Figures 8 and 9) and 30 psig pipeline pressure (60-ft. head) is:

$$
\begin{aligned}
\text { Power } & =5 \text { GPM } \times \text { Total Head }=5 \text { GPM }(8 \text { 1b./gal. }) \times(50+10+60) \mathrm{ft} . \\
& =4800 \mathrm{ft} .1 \mathrm{~b} / \mathrm{min} . \quad=100 \text { watts }
\end{aligned}
$$

At a pump efficiency of $50 \%$, primary pumping per 3 -ton water source heat pump is 200 watts. Secondary pumping has a much smaller head loss (less than $50 \mathrm{ft.}$ ), therefore pumping power input is assumed as 100 watts: The total pumping power is the total or 300 watts. This is the value used for performance and economic evaluations. Some evaluations also use 500 watts. To illustrate the comparison, water source heat pumps are designated as water source $\mathrm{A}(300)$ and water source $\mathrm{A}(500)$.

Performance and cost data are given in Table 40 for Meyer 6-inch SC submersible pumps. This is a commercially-rated submersible pump. For the economic evaluation, the 5-horsepower, 4-stage pump is utilized. The cost data are:

$$
\begin{array}{lr}
\text { Pump cost, 4-stage } & \$ 475.00 \\
\text { 5-horsepower, 3 phase, 230 volt } & \$ 1007.00 \\
\text { Control system (pressure aquated) } & \$ 195.00 \\
\hline \text { Total } & \$ 1677.00
\end{array}
$$

This is the suggested list price for a single unit delivered to an individual. Actual prices installed can be $30 \%$ to $50 \%$ under this figure. For the economic section, assuming numerous simultaneous installations, a cost of $\$ 1,500$ per well including piping, check valves, pressure release valve, grounding, etc., is used for the installed system. 
Table 40. Performance Tables for Submersible Pumps ${ }^{1}$

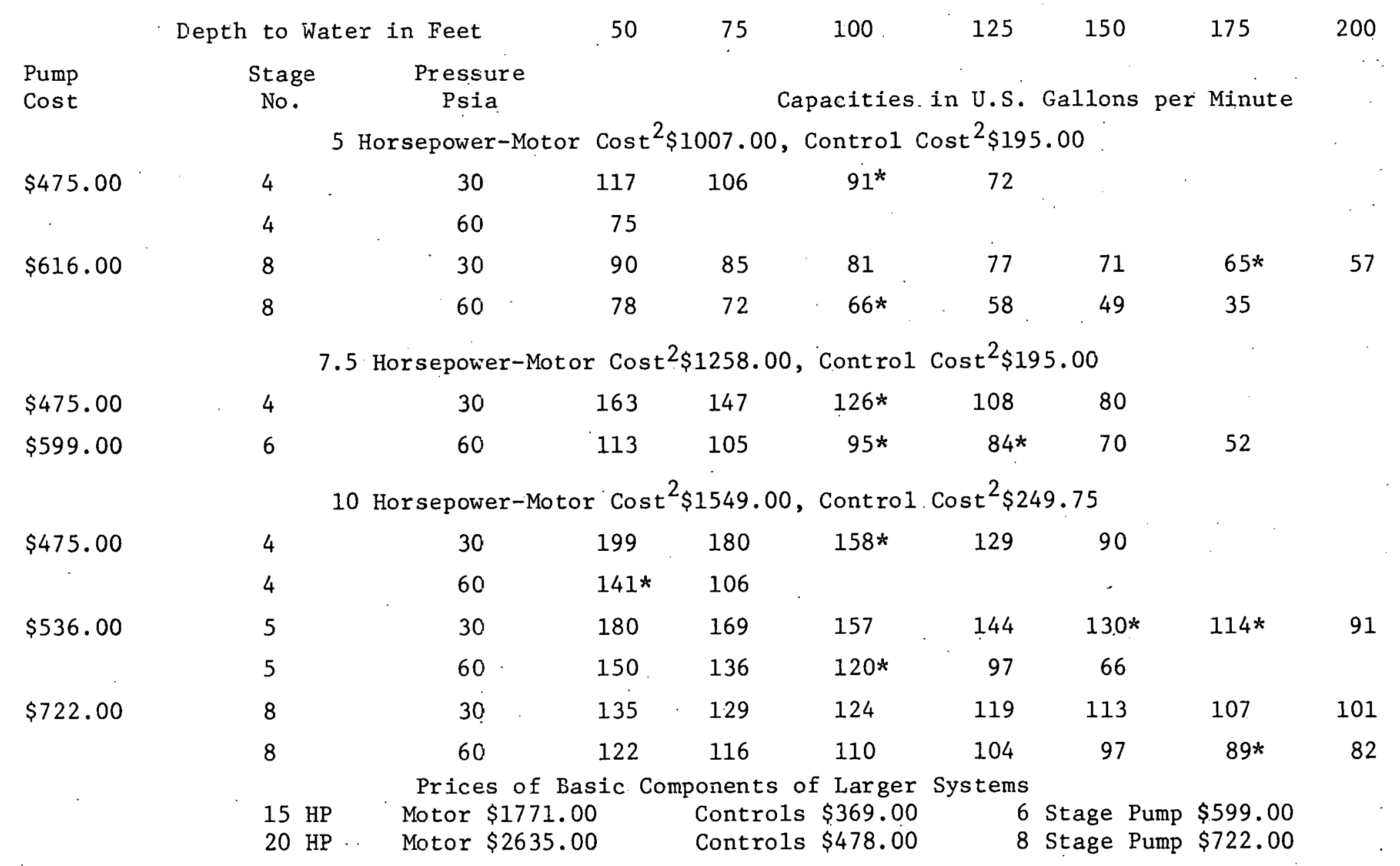

* Flow at peak efficiency

1. Data based on Meyer 6-inch SC submersible pumps (Price data for Fall 1978)

2. For 3 phase 230 volt service 
Wells can be constructed. to satisfy this system. As noted earlier, well costs will vary from area to area and can not be determined until the quality and extent of aquifers are known.

The capacitance tank at each well head will be in the 40 gal. to 200 gal. range. The 1978 price ranges on these tanks as provided by the Meyer Pump distributor are:

\section{Plain Tank Diaphragm Tank}

$\begin{array}{rlcc}42 & \text { gal. } & \$ 55 & \$-- \\ 120 \text { gal. } & 130 & 129 \\ 160 \text { gal. } & -- & 165\end{array}$

A diaphragm tank is recommended since a larger water volume can be utilized and an interface between air and water is eliminated. A cost of $\$ 130$ is used for economic analysis.

\section{IX.D. Water Distribution System}

The water distribution system transfers the water from the aquifers to the individual heat pumps in the home. The piping system must be both economical and compatible to the heat pump. Metal pipes such as steel or cast iron were eliminated on the basis of corrosion of dissimilar metals as well as economics. Copper and brass were eliminated primarily on an economic basis. As a result, polyvinyl chloride pipe is proposed as the distribution pipe.

The following specifications of polyvinyl chloride piping is a summary of material from the Piping Handbook. 25

Rigid, unplasticized polyvinyl chloride (PVC) is a thermoplastic material; it is tough and exceptionally resistant to chemical attack. Plastic pipe is generally available in iron pipe sizes (IPS). Typical sizes and weights for PVC are given in Table 41. 
Table 41. Normal Physical Properties of PVC Piping Materials

Materials

\section{Properties.}

Tensile strength, room temperature, psi . . . . Modulus of elasticity in tension, psi .....

Flexural strength, psi ... Izod impact strength, $\mathrm{ft}-1 \mathrm{~b} / \mathrm{in}$. of notch, notched, room temperature . . . . . . Izod impact strength; ft-lb/in. of specimen unnotched, room temperature . • . . . .

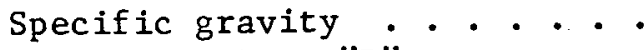
Hardness, Shore " $D$ " . . . . Heat distortion, temperature, F, at 264 psi . . . . . Coefficient of thermal conductivity, $\mathrm{BTU} /(\mathrm{sec}) \mathrm{ft} . \mathrm{F} \times 10^{4}$. . . . Specific heat, $\operatorname{cal} /(\mathrm{g} . \mathrm{C}) . . \cdot$ Coefficient of linear

thermal expansion per $\mathrm{C} \times 10^{5}$. Water absorption, \% in $24 \mathrm{hr}$. at $25 \mathrm{C}$
Type I

7,000

415,000

14,500

$0.5-1.0$

$10.0-19.0$

350,000

11,500

45

1.38

$78 / 82$

165

3.5

0.25

5

0.07
D790

4.5

0.25

ASTM

test no.

D638

D256

D256

D792

D785

D648

155

C-177

10

D696

0.07

D570 
PVC pipe is extruded. Fittings, flanges, and valves are manufactured by the injection-molding method which results in high density and complete homogeneity of the material. Three types of PVC piping are available.

Type I in the past has generally been marketed as "normal-impact" grade. It is now produced to a hydrostatic design stress of 2,000 psi for water at $73.4^{\circ} \mathrm{F}$. Two grades are recognized under the designations PVC 1120 and PVC 1220 .

Type II has been marketed as "high-impact" grade. Type II, Grade 1 is produced to a hydrostatic design stress of 1,000 psi for water at $73.4^{\circ} \mathrm{F}$ and is designated as PVC 2110 . It is also produced to a hydrostatic design stress of 1,250 psi and is designated as PVC 2112; material produced to a hydrostatic design stress of 1,600 psi is designated PVC 2116 .

Type IV is a newer grade produced to a hydrostatic design stress of 1,600 psi for water at $73.4^{\circ} \mathrm{F}$. It is designated as PVC 4116 .

Fittings and valves of Types I and II are readily available with threaded, solvent weld (socket), and flanged ends.

The 2 Type I PVC grades have greater strength properties over a wider temperature range. They can be used at temperatures up to $160^{\circ} \mathrm{F}$ and, in addition, at higher working pressures than can Type II PVC. These grades have superior chemical resistance throughout their temperature range.

Type I PVC grades should be specified in applications where greater strength, temperature resistance, or extreme chemical resistance is necessary. PVC is normally straw-colored without pigmentation. Industrial-process piping is usually colored dark gray for identification purposes but also may be produced as white, brown, red, or any other color. 
PVC pipe is covered by ASTM Standard D1785, Tentative Specifications for Poly (Vinyl Chloride) (PVC) Plastic Pipe: Voluntary industry stan dards have also been issued by the U.S. Department of Commerce: Commercial Standard CS256, Polyvinyl Chloride (PVC) Plastic Pipe (SDR-PR and Class T) and Commercial Standard CS207, Rigid Unplasticized Polyvinyl Chloride Pipe. The normal physical properties of PVC are summarized in Table 42. PVC piping is extensively used in highly corrosive applications involving acids, alkalies, salt solutions, alcohols, and many types of chemicals. PVC piping is also used in oil fields because it can carry sour crude oil to which PVC is chemically inert and because paraffin build-up is minimum on the smooth inside surfaces of this pipe. Other applications include salt-water disposal in oil fields and gas transmission service. PVC will handle most chemicals up to $150^{\circ} \mathrm{F}$. Still other applications include the piping of cold water in industrial plants because PVC is nontoxic and will not impart odor or taste to the water, and also vent piping for the removal of acid fumes and corrosive gases from industrial plants.

PVC pipe and fittings experience little or no physical deterioration when exposed to direct sunlight; sunlight causes deterioration in severai other plastic piping materials. Like other plastic materials, PVC wili not produce sparks when struck. PVC is safe to use around explosives or flammable vapors, and it does not support combustion. Also, water contamInants do not build up on the smooth walls of PVC pipe or fittings.

Because polyvinyl chloride, like most other plastic materials, is somewhat notch-sensitive; maximum allowable working pressures for threaded pipe are considerably lower than those for unthreaded pipe. The strength of polyvinyl chloride decreases as the operating temperature increases, 
Table 42. Commercial Sizes (IPS) and Weights of Polyvinyl Chloride (PVC) Pipe (Ahstracted from ASTM Specification D1785-64T)

\begin{tabular}{|c|c|c|c|c|c|c|c|}
\hline \multirow{2}{*}{$\begin{array}{l}\text { Nominal } \\
\text { size, } \\
\text { in. }\end{array}$} & \multirow[t]{2}{*}{ Schedule } & \multirow{2}{*}{$\begin{array}{c}\text { Wall } \\
\text { thick- } \\
\text { ness, **in. }\end{array}$} & \multirow[t]{2}{*}{$\begin{array}{l}\text { OD, } \\
\text { in. }\end{array}$} & \multirow[t]{2}{*}{$\begin{array}{l}\text { ID, } \\
\text { in. }\end{array}$} & \multirow{2}{*}{$\begin{array}{c}\text { Theoreti- } \\
\text { cal } \\
\text { weight, * } \\
\text { lb/ft. }\end{array}$} & \multicolumn{2}{|c|}{$\begin{array}{l}\text { Calculated min } \\
\text { bursting pressure, } \\
\text { psi }\end{array}$} \\
\hline & & & & & & Note 1 & Note 2 \\
\hline $1 / 4$ & $\begin{array}{l}40 \\
80\end{array}$ & $\begin{array}{l}0.088 \\
0.119\end{array}$ & $\begin{array}{l}0.540 \\
0.540\end{array}$ & $\begin{array}{l}0.364 \\
0.302\end{array}$ & $\begin{array}{l}0.076 \\
0.096\end{array}$ & $\begin{array}{l}2,490 \\
3.620\end{array}$ & $\begin{array}{l}1,950 \\
2,830\end{array}$ \\
\hline $1 / 2$ & $\begin{array}{l}40 \\
80\end{array}$ & $\begin{array}{l}0.109 \\
0.147\end{array}$ & $\begin{array}{l}0.840 \\
0.840\end{array}$ & $\begin{array}{l}0.622 \\
0.546\end{array}$ & $\begin{array}{l}0.153 \\
0.195\end{array}$ & $\begin{array}{l}1,910 \\
2,720\end{array}$ & $\begin{array}{l}1,490 \\
2,120\end{array}$ \\
\hline $3 / 4$ & $\begin{array}{l}40 \\
80\end{array}$ & $\begin{array}{l}0.113 \\
0.154\end{array}$ & $\begin{array}{l}1,050 \\
1.050\end{array}$ & $\begin{array}{l}0.824 \\
0.742\end{array}$ & $\begin{array}{l}0.203 \\
0.265\end{array}$ & $\begin{array}{l}1,540 \\
2,200\end{array}$ & $\begin{array}{l}1,210 \\
1,720\end{array}$ \\
\hline 1 & $\begin{array}{r}40 \\
.80\end{array}$ & $\begin{array}{l}0.133 \\
0.179\end{array}$ & $\begin{array}{l}1.315 \\
1.315\end{array}$ & $\begin{array}{l}1.049 \\
0.957\end{array}$ & $\begin{array}{l}0.305 \\
0.385\end{array}$ & $\begin{array}{l}1,440 \\
2,020\end{array}$ & $\begin{array}{l}1,130 \\
1,580\end{array}$ \\
\hline $11 / 4$ & $\begin{array}{l}40 \\
80\end{array}$ & $\begin{array}{l}0.140 \\
0.191\end{array}$ & $\begin{array}{l}1.660 \\
1.660\end{array}$ & $\begin{array}{l}1.380 \\
1.278\end{array}$ & $\begin{array}{l}0.409 \\
0.550\end{array}$ & $\begin{array}{l}1,180 \\
1,660\end{array}$ & $\begin{array}{r}920 \\
1,300\end{array}$ \\
\hline $11 / 2$ & $\begin{array}{l}40 \\
80\end{array}$ & $\begin{array}{l}0.145 \\
0.200\end{array}$ & $\begin{array}{l}1.900 \\
1,900\end{array}$ & $\begin{array}{l}1.610 \\
1.500\end{array}$ & $\begin{array}{l}0.489 \\
0.653\end{array}$ & $\begin{array}{l}1,060 \\
1,510\end{array}$ & $\begin{array}{r}830 \\
1,180\end{array}$ \\
\hline 2 & $\begin{array}{l}40 \\
80\end{array}$ & $\begin{array}{l}0.154 \\
0.218\end{array}$ & $\begin{array}{l}2.375 \\
2.375\end{array}$ & $\begin{array}{l}2.067 \\
1.939\end{array}$ & $\begin{array}{l}0.640 \\
0.910\end{array}$ & $\begin{array}{r}800 \\
1,290\end{array}$ & $\begin{array}{r}690 \\
1,010\end{array}$ \\
\hline 3 & $\begin{array}{l}40 \\
80\end{array}$ & $\begin{array}{l}0.216 \\
0.300\end{array}$ & $\begin{array}{l}3.500 \\
3.500\end{array}$ & $\begin{array}{l}3.068 \\
2.900\end{array}$ & $\begin{array}{l}1.380 \\
1.845\end{array}$ & $\begin{array}{r}840 \\
1,200\end{array}$ & $\begin{array}{l}660 \\
940\end{array}$ \\
\hline 4 & $\begin{array}{l}40 \\
80\end{array}$ & $\begin{array}{l}0.237 \\
0.337\end{array}$ & $\begin{array}{l}4.500 \\
4.500\end{array}$ & $\begin{array}{l}4.026 \\
3.826\end{array}$ & $\begin{array}{l}1.965 \\
2.710\end{array}$ & $\begin{array}{r}710 \\
1,040\end{array}$ & $\begin{array}{l}560 \\
810\end{array}$ \\
\hline
\end{tabular}

*These representative values are not specified in ASTM D1785-64T. **Thicknesses listed are minimum values. Tolerance is generally $-0+10$ per cent. Note 1. Materials are PVC 1120, 1220, and 4116. A fiber stress of 6,400 psi was used in bursting pressure calculations.

Note 2. Materials are PVC 2112, 2116, and 2120. A fiber stress of 5,000 psi was used in bursting pressure calculations. 
and allowable working pressures must be decreased at higher temperatures. Working pressures at $75^{\circ} \mathrm{F}$ are approximately $20 \%$ of the bursting

pressures. When operating temperatures exceed $75^{\circ} \mathrm{F}$, the maximum operating pressure for Type I pipe may be determined from Figure 31.

Type I is being considered for the piping system.

Cost analysis has been made for the various system alternatives shown in Section VII : (Figures 17 to 20 ). The results are listed in Table 43. The prime costs are for pipes, pipe installation, and insurlation.

The 2-pipe system has been selected on the basis of simplicity, performance, and cost. The 1-pipe system, though more economical, penalizes customers down the line with reduced performance. It also reduces performance with simultaneous heating and cooling.

The pressure losses for various pipe diameters and flow rates are shown in Figures 8 and 9. A modified computer code (Appendix II) for calculating pipe-pressure loads was used for the analysis. Laminar flow is assumed in the piping which is reasonable for the smooth pipe with limited flow. The system assumes each home receives the allotted water flow rate, thereby reducing the flow. This occurs at the given separation distances noted on the curve for 2 homes at each cutoff. The totai. pressure loss is the pressure loss through the 10 increments. Using pipe diameter of over 4 inches keeps the pressure losses at negligible values and allows flow both ways between the pipes. Change in elevation will not effect the pressure between pipes as they run parallel and adjacent. Connections between the main lines and homes is to be made with $1 \frac{1}{2}-$ inch or 2-inch pipe. This will keep the pressure loss for flows up to 20 


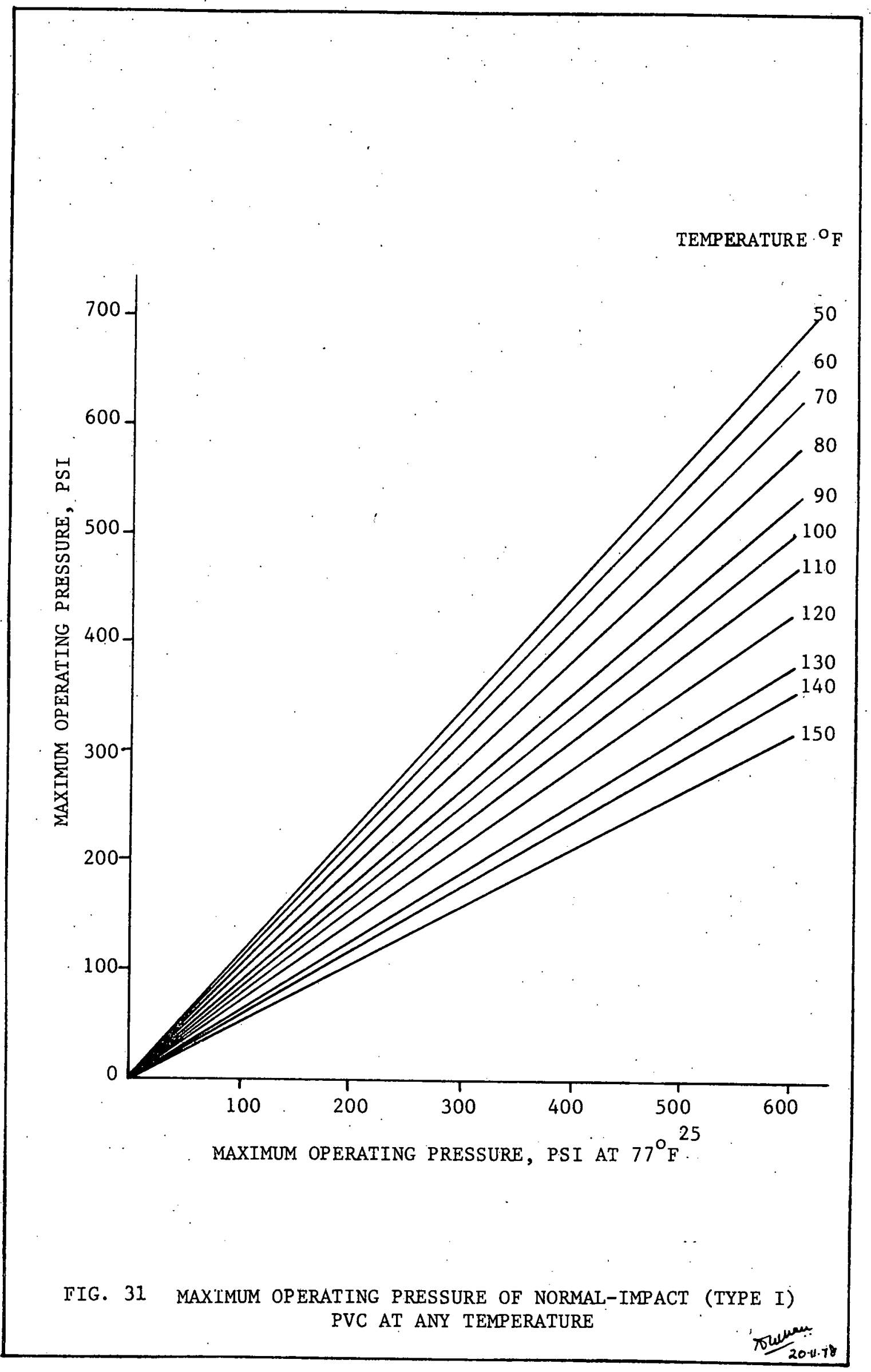


Table 43..$^{6}$ COST OF DISTRIBUTION PIPE ${ }^{1}$, INSTALLATION ${ }^{2}$, AND TRENCHING ${ }^{3}$

\begin{tabular}{|c|c|c|c|c|}
\hline \multirow{3}{*}{$\begin{array}{l}\text { DIAMETER } \\
\text { (INCHES) }\end{array}$} & \multirow{2}{*}{\multicolumn{2}{|c|}{ UNINSULATED }} & \multirow[b]{2}{*}{ PIPE } & \multirow[t]{2}{*}{ PIPE COST PER LINEAR } \\
\hline & & & & \\
\hline & 1 & 2 & 3 & 2 INSULATED; 1 UNINS \\
\hline $1 \frac{1}{2}$ & 1.70 & 3.40 & 5.10 & $8.10+1.70=9.80$ \\
\hline 2 & 2.05 & 4.10 & 6.15 & $8.90+2.05=10.95$ \\
\hline 4 & 3.20 & 6.40 & 9.60 & $14.30+3.20=17.50$ \\
\hline 6 & 4.60 & 9.20 & 13.80 & $23.70+4.60=28.30$ \\
\hline 8 & 7.25 & 14.50 & 21.75 & $32.10+7.25=39.35$ \\
\hline
\end{tabular}

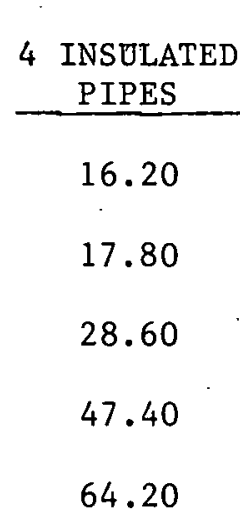

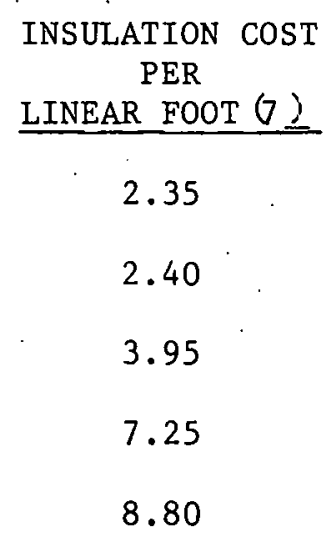

TRENCHING COST PER

LINEAR FOOT

EXCAVATION (4) BACKF ILL. (5)

1.110 .37

1.11

0.37

1.11

0.37

1.11

0.37

1.11

0.37

1. The costs stated above are for Polyvinyl Chloride (PVC) pipe, class 160, SDR 26.

2. The pipe cost stated above includes material, installation, and subcontractors overhead and profit.

3. The trenching costs are stated for a 4 . foot wide, 3 foot deep trench which would accomodate any combination of pipes.

4. This cost is for the use of a $\frac{1}{2}$ cubic yard tractor backhoe.

5. This figure is made on the basis of using a dozer with up to a 300 foot hard distance and no compaction.

6. All the figures stated above were taken from and derived from those given in Means' Building Construction Cost Data.

7. The costs for the $1 \frac{1}{2} "$ and $2 "$ insulation is for $\frac{1}{2} "$ thick foam rubber sleeves and the cost of the 4 " to 8 " sizes is for $2^{\prime \prime}$ thick fiberglass insulation. 
GPM below $15 \mathrm{ft}$. of water. A 1/12-horsepower pump (100 watts) can be used to circulate the water between the main water lines and the individual heat pumps. This type system is standard on numerous solar systems. IX.E. Heat Exchanger Fouling

In many present day water source heat pump systems, the water is circulated in a closed loop. The water is cooled in a cooling tower and heated by waste heat or in some cases directly from other energy sources. Mineral content and $\mathrm{pH}$ are closely controlled. In the proposed system, water in the aquifers and mineral additions during water circulation must be evaluated.

Knudson has been active in condenser water fouling research for many years. 28129 Basic premise is that mineral buildup occurs continuously, but erosion of the mineral buildup also occurs continuously. After a period of time the mineral buildup and erosion reach equilibrium. This is demonstrated by plotting fouling resistance " $R_{f}$ ", the change in the heat transfer coefficient versus time. In general the time to equilibrium varies from 150 to 600 hours. Typical curves are shown in Figure 32 . Knudson's series of papers have shown the variations for a number of minerals. Table' 44 shows a typical mineral content for a series of runs with various salts and changing $\mathrm{pH}$. Tables 45 and 46 show typical mineral contents in ground water in certain formations in Alabama. Table 45 shows relatively low levels, and Table 46 shows relatively high levels. Knudson's test data and that for many aquifers cannot be compared directly, however, maximum hardness values may be of similar magnitude.

In his papers, Knudson's maximum value of resistance fouling is of the order of magnitude of $0.0004 \mathrm{ft} .{ }^{2} \mathrm{hr} .{ }^{\mathrm{o}} \mathrm{F} / \mathrm{Btu}$. The value for sea water 


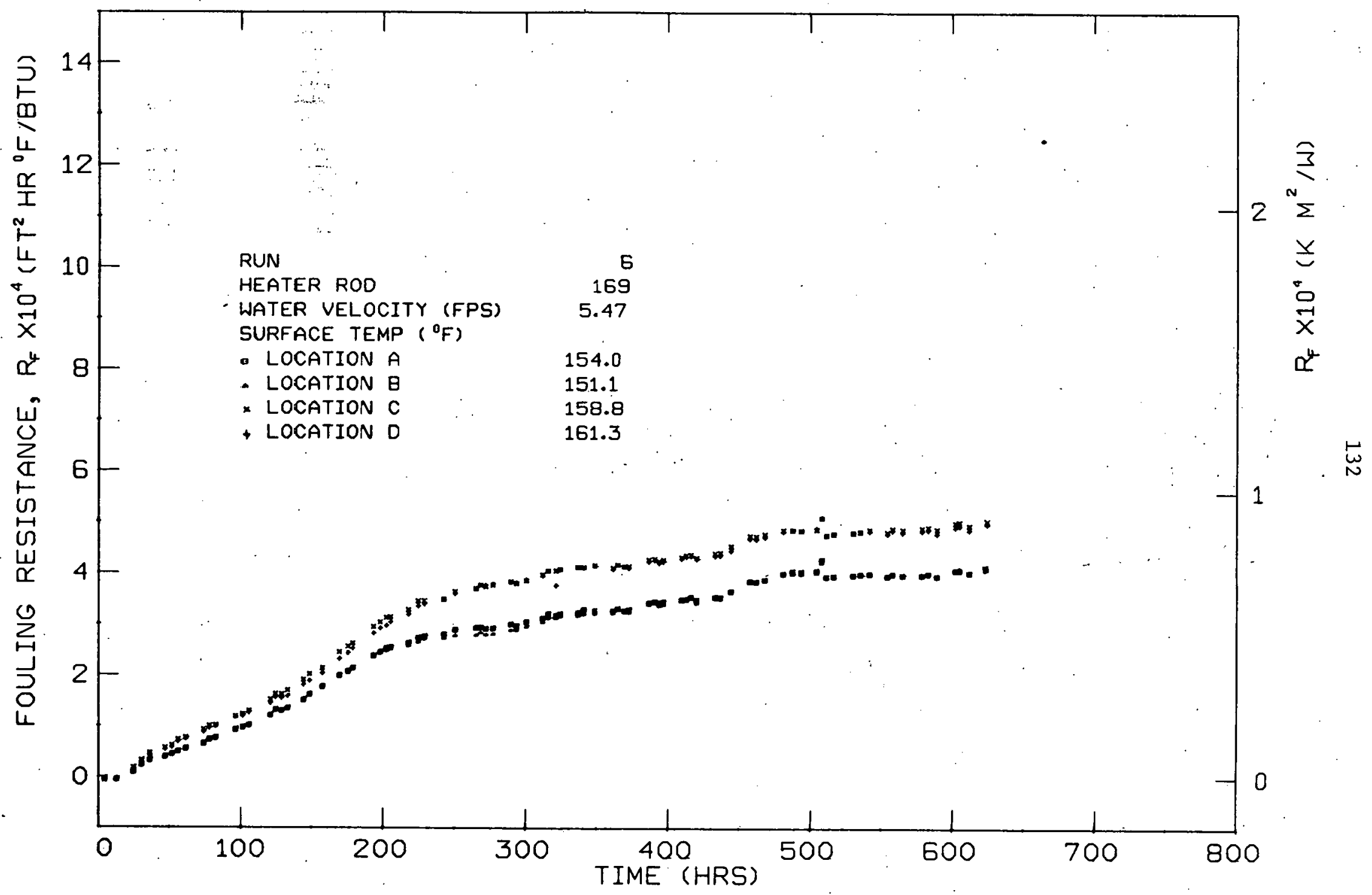

FIGURE 32. TIME EFFECT ON HEAT EXCHANGER FOULING BY LEE AND KNUDSON 
30

Table 44. Average Cooling Tower Water Quality, Runs 6-20

\begin{tabular}{|c|c|c|c|c|c|c|c|}
\hline Run & $\begin{array}{l}\text { Total } \\
\text { Hardness } \\
\left(\text { ppm } \mathrm{CaCO}_{3}\right)\end{array}$ & $\begin{array}{l}\mathrm{Ca} \\
\text { Hardness } \\
\left(\mathrm{ppm} \mathrm{CaCO}_{3}\right)\end{array}$ & $\begin{array}{l}\text { m-alkalinity } \\
\left(\mathrm{ppm} \mathrm{CaCO}_{3}\right)\end{array}$ & $\begin{array}{l}\text { k-alkalinity } \\
\left(\mathrm{ppm} \mathrm{CaCO}_{3}\right)\end{array}$ & $\begin{array}{l}\mathrm{C} 1 \\
(\mathrm{ppm} \\
\mathrm{NaC1})\end{array}$ & $\mathrm{pH}$ & $\begin{array}{l}\mathrm{TS} \\
\text { (ppm) }\end{array}$ \\
\hline 6 & $\begin{array}{c}219 \\
(7.8)\end{array}$ & $\begin{array}{c}152 \\
(4.2)\end{array}$ & $\begin{array}{c}190 \\
(16.1)\end{array}$ & $\begin{array}{c}23 \\
(2.2)\end{array}$ & $\begin{array}{c}243 \\
(22.3)\end{array}$ & $\begin{array}{c}8.97 \\
(0.10)\end{array}$ & $\begin{array}{l}613 \\
(47)\end{array}$ \\
\hline 7 & $\begin{array}{l}217 \\
(4.9)\end{array}$ & $\begin{array}{l}151 \\
(2: 4)\end{array}$ & $\begin{array}{l}1.91 \\
(6.9)\end{array}$ & $\begin{array}{l}20 \\
(1.5)\end{array}$ & $\begin{array}{c}216 \\
(6.3)\end{array}$ & $\begin{array}{l}8.90 \\
(0.05)\end{array}$ & $\begin{array}{l}568 \\
(25)\end{array}$ \\
\hline 8 & $\begin{array}{c}221 \\
(9.3)\end{array}$ & $\begin{array}{c}154 \\
(5.5)\end{array}$ & $\begin{array}{c}206 \\
(15.1)\end{array}$ & $\begin{array}{l}21 \\
(1.3)\end{array}$ & $\begin{array}{c}221 \\
(10.1)\end{array}$ & $\begin{array}{c}8.89 \\
(0.05)\end{array}$ & $\begin{array}{l}575 \\
(35)\end{array}$ \\
\hline 9,10 & $\begin{array}{l}216 \\
(8.3)\end{array}$ & $\begin{array}{l}150 \\
(2.7)\end{array}$ & $\begin{array}{l}183 \\
(8.9)\end{array}$ & $\begin{array}{l}23 \\
(1.2)\end{array}$ & $\begin{array}{c}243 \\
(16.3)\end{array}$ & $\begin{array}{c}9.01 \\
(0.10)\end{array}$ & $\begin{array}{l}604 \\
(32)\end{array}$ \\
\hline 11,12 & $\begin{array}{c}218 \\
(5.6)\end{array}$ & $\begin{array}{c}151 \\
(3.3)\end{array}$ & $\begin{array}{c}182 \\
(10.9)\end{array}$ & $\begin{array}{l}25 \\
(1.5)\end{array}$ & $\begin{array}{c}266 \\
(8.8)\end{array}$ & $\begin{array}{c}9.03 \\
(0.07)\end{array}$ & $\begin{array}{l}657 \\
(28)\end{array}$ \\
\hline 13,14 & $\begin{array}{c}218 \\
(7.8)\end{array}$ & $\begin{array}{c}149 \\
(6.1)\end{array}$ & $\begin{array}{c}194 \\
(8.5)\end{array}$ & $\begin{array}{l}25 \\
(1.8)\end{array}$ & $\begin{array}{r}297 \\
(15.9)\end{array}$ & $\begin{array}{c}9.00 \\
(0.04)\end{array}$ & $\begin{array}{l}677 \\
(22)\end{array}$ \\
\hline 15 & $\begin{array}{c}218 \\
(9.1)\end{array}$ & $\begin{array}{c}148 \\
(5.6)\end{array}$ & $\begin{array}{c}210 \\
(20.4)\end{array}$ & $\begin{array}{l}27 \\
(2.4)\end{array}$ & $\begin{array}{c}299 \\
(15.5)\end{array}$ & $\begin{array}{c}9.02 \\
(0.05)\end{array}$ & $\begin{array}{l}683 \\
(45)\end{array}$ \\
\hline 16,17 & $\begin{array}{c}218 \\
(10.3)\end{array}$ & $\begin{array}{l}148 \\
(4.9)\end{array}$ & $\begin{array}{c}228 \\
(15.8)\end{array}$ & $\begin{array}{l}29 \\
(2.5)\end{array}$ & $\begin{array}{r}301 \\
(14.9\end{array}$ & $\begin{array}{c}9.05 \\
(0.06)\end{array}$ & $\begin{array}{l}691 \\
(59)\end{array}$ \\
\hline 18,19 & $\begin{array}{c}222 \\
(9.7)\end{array}$ & $\begin{array}{c}152 \\
(4.6)\end{array}$ & $\begin{array}{c}253 \\
(14: 7)\end{array}$ & $\begin{array}{l}35 \\
(1.2)\end{array}$ & $\begin{array}{l}320 \\
(9.8)\end{array}$ & $\begin{array}{c}9.09 \\
(0.05)\end{array}$ & $\begin{array}{l}745 \\
(68)\end{array}$ \\
\hline 20 & $\begin{array}{c}223 \\
(8.5)\end{array}$ & $\begin{array}{c}151 \\
(2.3)\end{array}$ & $\begin{array}{c}251 \\
(12.7)\end{array}$ & $\begin{array}{c}35 \\
(1.2)\end{array}$ & $\begin{array}{c}316 \\
(10.4)\end{array}$ & $\begin{array}{c}9.09 \\
(0.03)\end{array}$ & $\begin{array}{l}725 \\
(47)\end{array}$ \\
\hline
\end{tabular}




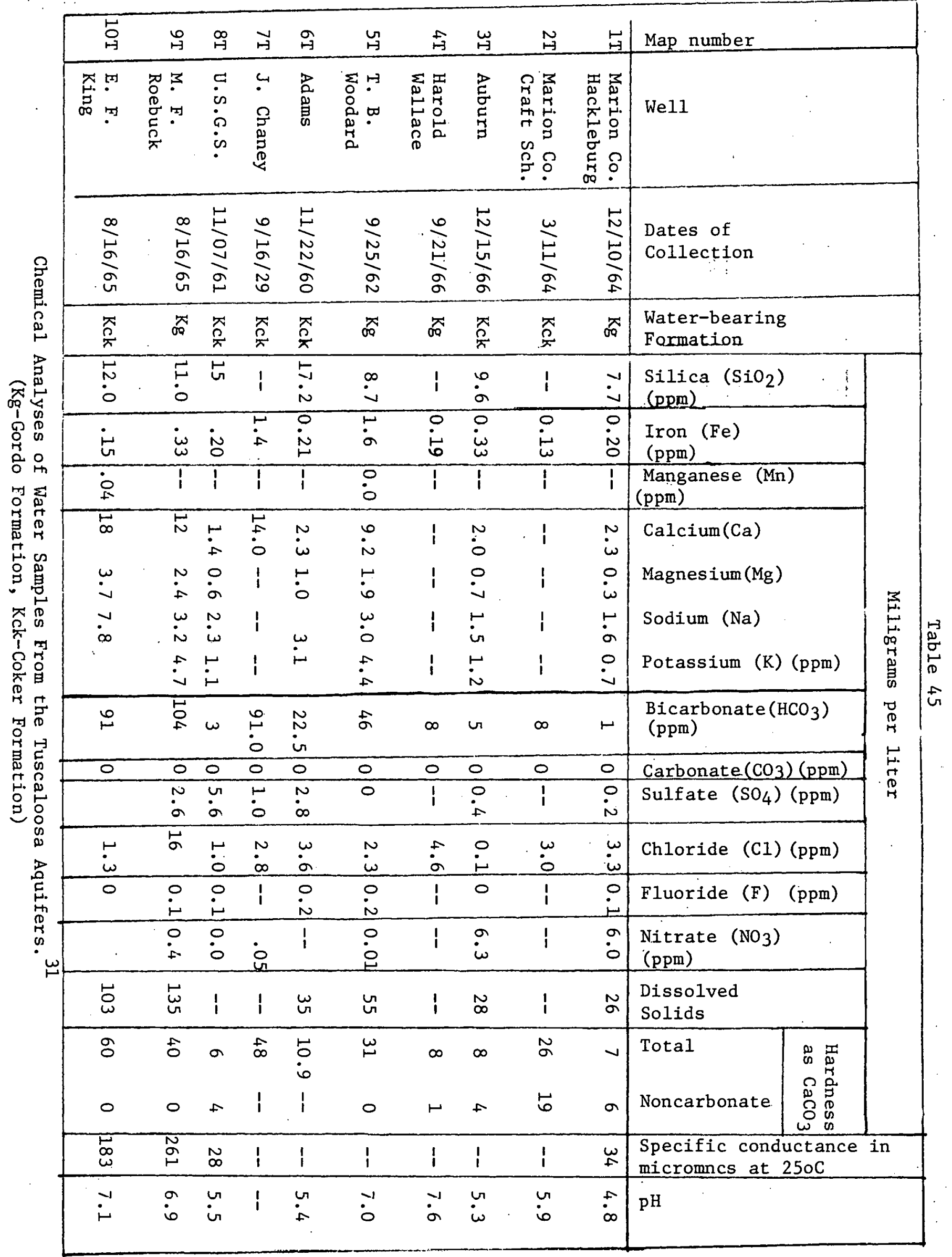




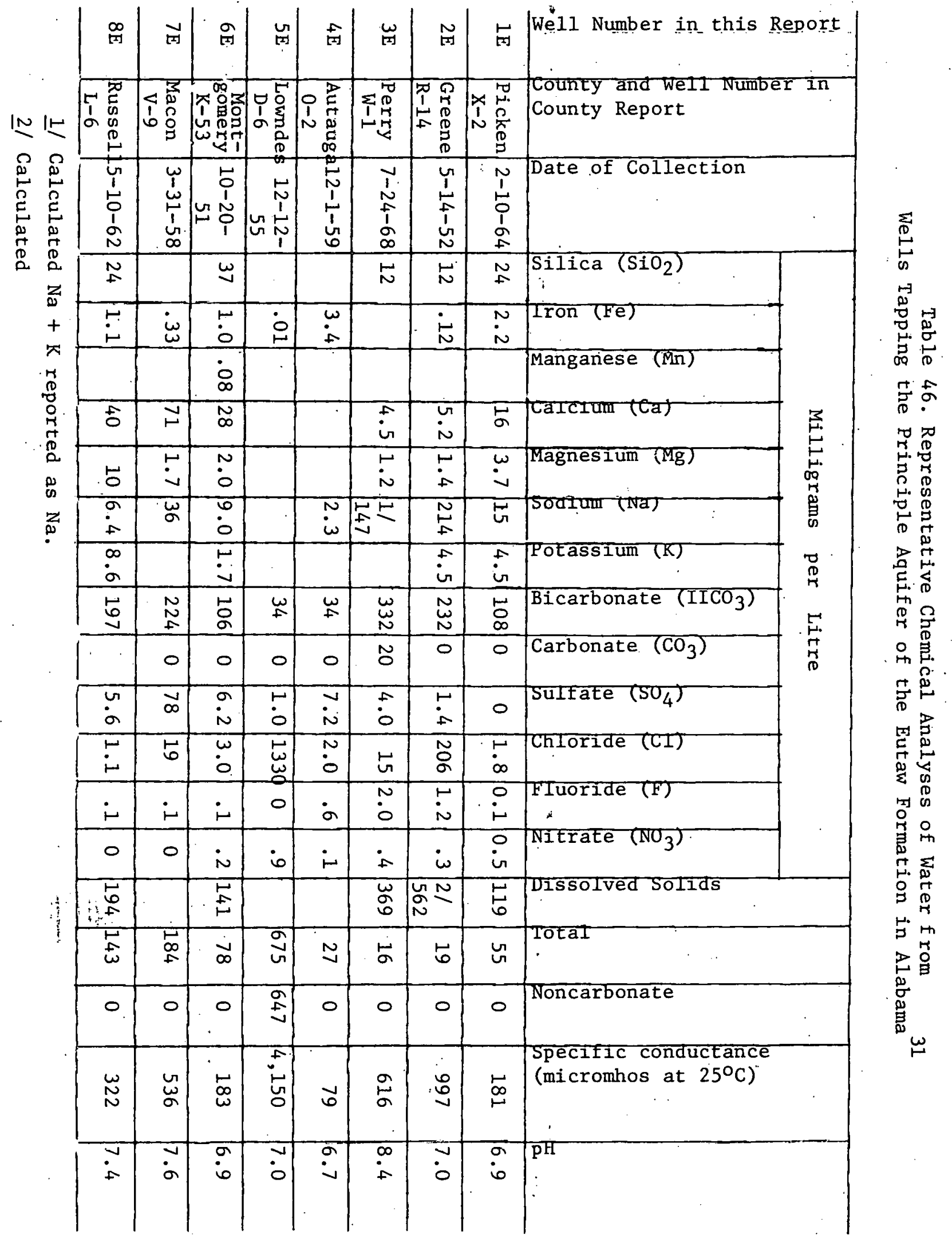


is given as $0.0005 \mathrm{ft} .{ }^{2} \mathrm{hr}{ }^{\circ} \mathrm{F} / \mathrm{Btu}$. The effect of this level is shown to be minor.

The general range of heat transfer coefficients for a Freon 12 condensor is 80 to $150 \mathrm{Btu} / \mathrm{ft} .{ }^{2} \mathrm{hr}:{ }^{\circ} \mathrm{F}$. The reciprocal or resistance therefore varies from 0.012 to $0.00667 \mathrm{ft} .{ }^{2} \mathrm{hr} .{ }^{\circ} \mathrm{F} / \mathrm{Btu}$. The change due to mineral fouling is approximately $1 \%$. This is not a major problem.

Possibilities of heat exchanger fouling and aquifer clogging resulting from biological growth must be evaluated in the laboratory or in demonstration projects. These variables can be controlled, if necessary, by the addition of chemical inhibitors.

The problem of aquifer clogging resulting from solution and precipitation of minerals in small openings can be minimized by controlling the $\mathrm{pH}$ level. The velocity levels in most aquifers is very small. Therefore the fouling of heat exchangers is expected to be minimal.

IX.F. Solar Energy Addition

The system makes possible an economical method of utilizing solar energy to help heat buildings. A large portion of the continental United States requires much larger quantities of energy for heating than cooling. If waste heat is not available or the proper mixing of buildings is not practicable, solar energy is an alternative. With annual thermal storage and the relatively low collection temperatures, basic swimming pool heaters are a possibility. Summer usage eliminates the freezing problem and the requirement for glazing thereby allows direct system water circulation. Table 47 gives the horizontal solar energy collection quantities for the summer months per $100 \mathrm{ft} .{ }^{2}$ of collector area. Collection efficiences are based on Figure 33 where the June through August solar energy 
TABLE 47 SOLAR ENERGY COLLECTIONS ON 100-SQUARE FOOT HORIZONTAL COLLECTOR*

\begin{tabular}{lllllllc}
\multicolumn{1}{c}{ CITIES } & APRIL & MAY & JUNE & JULY & AUGUंST & SEPTEMBER & $\begin{array}{c}\text { JUNE-AUGUST } \\
\text { OVER ALL }\end{array}$ \\
Birmingham, AL & & & & & & & \\
Tuscon, AZ & 3301 & 3708 & 3803 & 3782 & 3337 & 2870 & 10922 \\
Los Angeles, CA & 4306 & 5191 & 5095 & 4746 & 4227 & 3588 & 14068 \\
Denver, CO & 3588 & 4078 & 3947 & 4078 & 3708 & 3229 & 11733 \\
Miami, FL & 3660 & 4449 & 4593 & 4449 & 4004 & 3229 & 13046 \\
Atlanta, GA & 3660 & 3782 & 3660 & 3708 & 3337 & 2870 & 13575 \\
Chicago, IL & 3373 & 3708 & 3732 & 3856 & 3337 & 2870 & 10925 \\
New Orleans, LA & 2942 & 3708 & 3947 & 4078 & 3337 & 2512 & 11362 \\
Boston, MA & 3157 & 3708 & 3660 & 3337 & 3337 & 2870 & 10334 \\
Detroit, MI & 2942 & 3559 & 3947 & 3782 & 3337 & 2583 & 11066 \\
Saint Louis, MO & 2727 & 3337 & 3732 & 3856 & 3337 & 2153 & 10925 \\
New York, NY & 3086 & 3782 & 4019 & 4078 & 3634 & 2799 & 11731 \\
Akron, OH & 3014 & 3708 & 3947 & 3708 & 3337 & 2655 & 10992 \\
Tulsa, OK & 2799 & 3337 & 3947 & 4078 & 3337 & 2511 & 11362 \\
Portland, OR & 3516 & 4004 & 4234 & 4449 & 3856 & 3086 & 12539 \\
Philadelphia, PA & 2942 & 3708 & -3947 & 3708 & 2966 & 2512 & 10621 \\
Providence, RI & 3014 & 3708 & 3947 & 3708 & 3337 & 2655 & 10992 \\
Columbus, SC & 2942 & 3708 & -3947 & 3782 & 3337 & 2583 & 11066 \\
Nashvilie, TN & 3445 & 3708 & 3588 & 3708 & 3337 & 2870 & 10633 \\
Houston, TX & 3157 & 3708 & 3947 & 4004 & 3337 & 2870 & 11288 \\
Salt Lake City, UT & 3373 & 3708 & 3732 & 3708 & 3708 & 3014 & 11148 \\
Seattle, WA. & 3588 & 4449 & 4664 & 4597 & 4078 & 3229 & 13339 \\
Milwauke, WI & 2870 & 3708 & 3732 & 3782 & 2966 & 2512 & 10480 \\
& 2870 & 3559 & 3588 & 3856 & 3337 & 2153 & 10781
\end{tabular}

* Based on manufactors test data Average efficiency 0.65

No glazing 
Average flow rate $3.7 \mathrm{GPM}$

Average Wind Velocity $3.7 \mathrm{MPH}$

$I$ = Solar radiation on the horizontal surface. Ptu/hr-ft

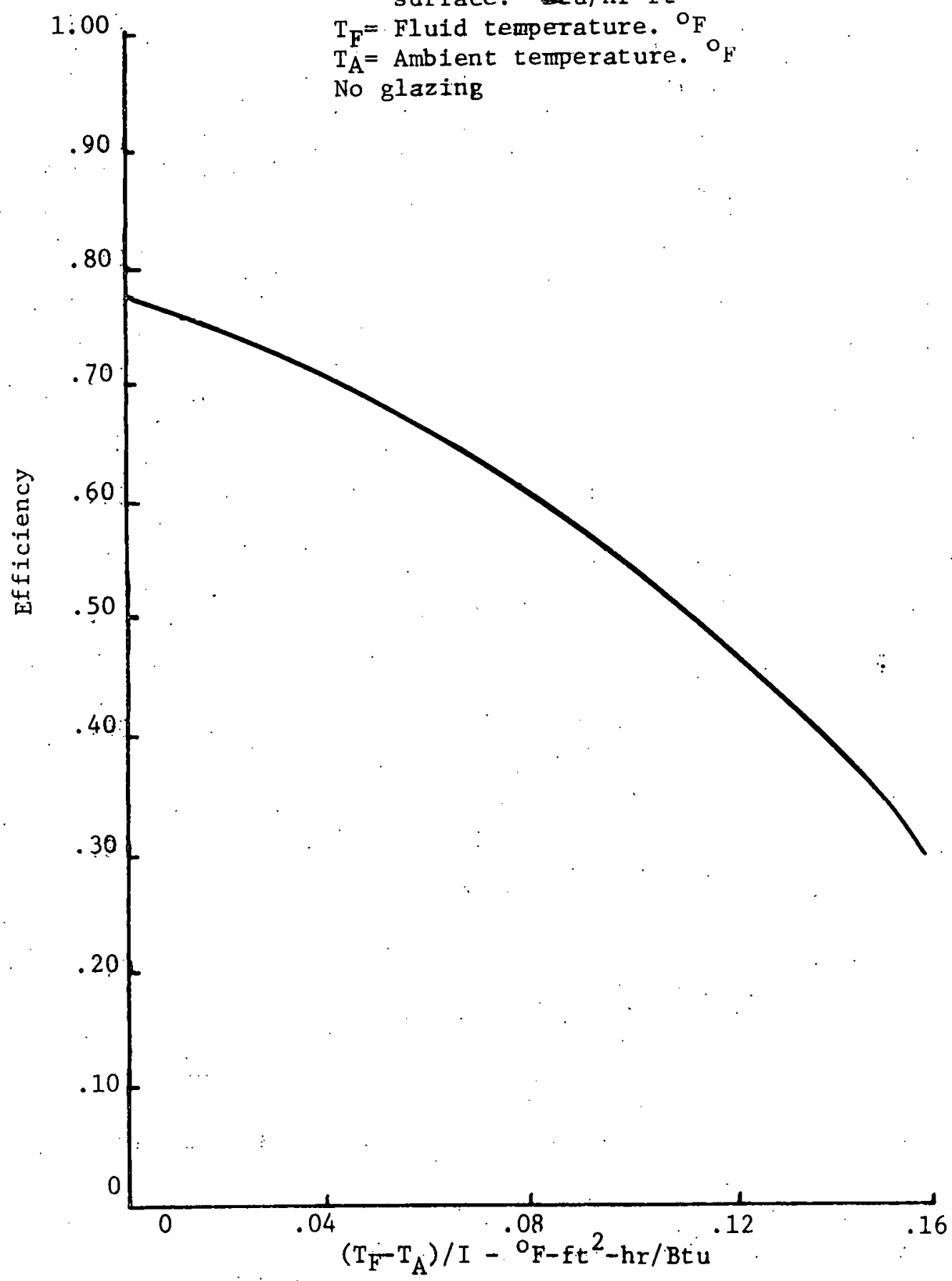

39

FIGURE $33^{\circ}$ Measured Efflciency of Solar Collector 


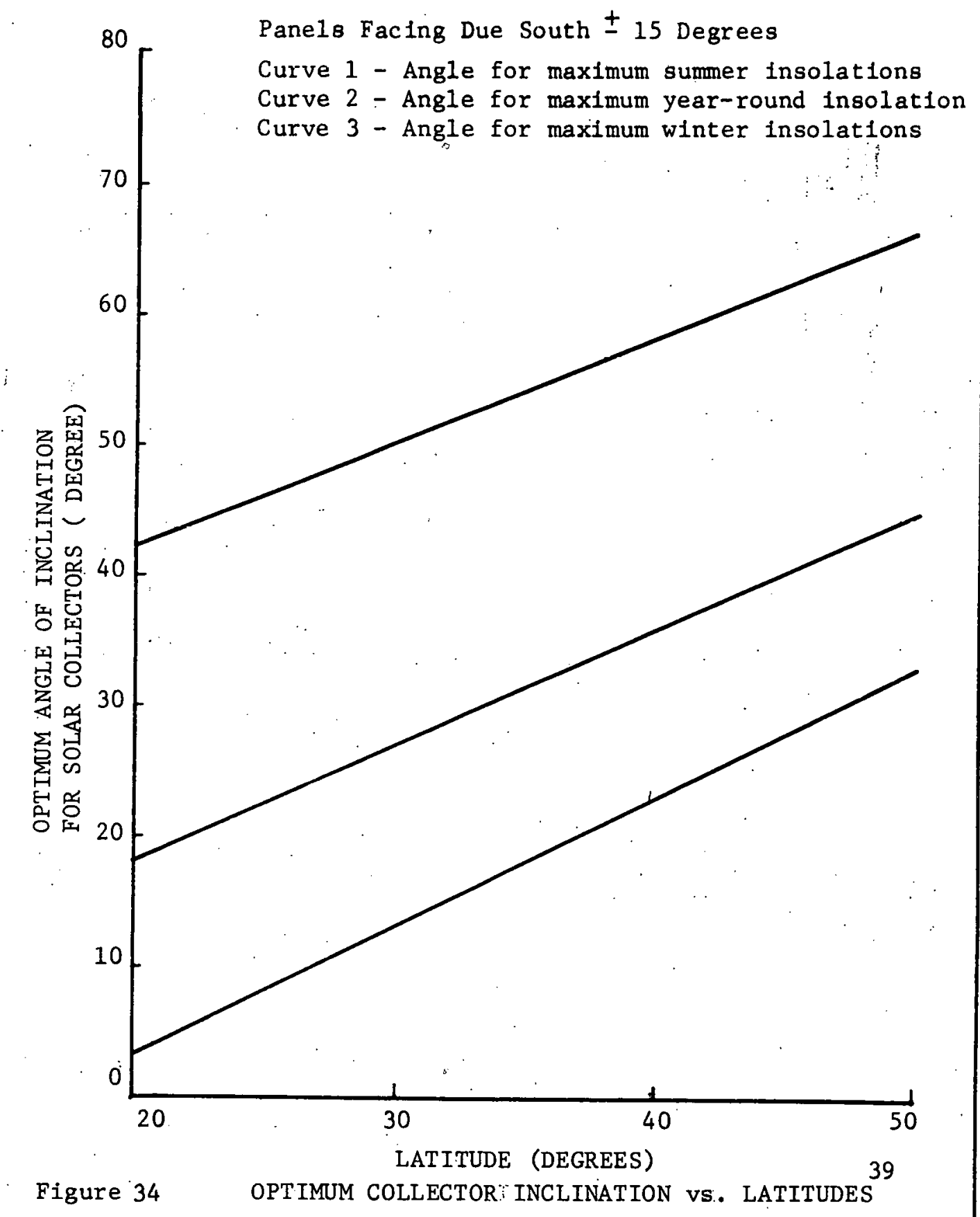


39

total is also shown. An increase in performance will be made by inclining the collectors. Optimum angle of inclination is shown in Figure 34 for various time periods.

In a community energy system, solar collectors are not required on each building or surface area. They can be installed, operated, and serviced on a building with optimum locations. Placing the panels on a roof helps the occupant by decreasing the heating and cooling loads without involving any inconvenience. Figure 35 shows a typical installation.

Figure 36 shows schematically a hard rubber solar collector without glazing, and Figure 37 shows schematically a black bag solar collector. 34,35 Both collectors retail between $\$ 2.50$ and $\$ 3.50$ per $\mathrm{ft}^{2}$ and are designed for roof collection. Figure 38 shows a proposed solar pond collector and an estimation of installed costs with required pump and controls. 36 For the economic analysis, an installed $100^{\circ} \mathrm{ft} .{ }^{2}$ panel is included for energy addition at a cost of $\$ 3.00 / \mathrm{ft} .^{2}$ or $\$ 3,000$ total. It must be emphasized that this would be used only if waste heat is not available at a competitive cost.

IX.G. Minor Related Systems

Providing water for fires is a definite potential of the system. In general, water requirements are determined by insurance underwriters and vary neighborhood-by-neighborhood with no set standards. Commonly residential requirements vary from 900 GPM to $2500 \mathrm{GPM}$. A mall or major building demands about ,4000 GPM. City water lines in Tuscaloosa, Alabama, are predominately 8 -inch and 6-inch lines for distribution to provide fire protection. Without fire protection requirements these would nor- 


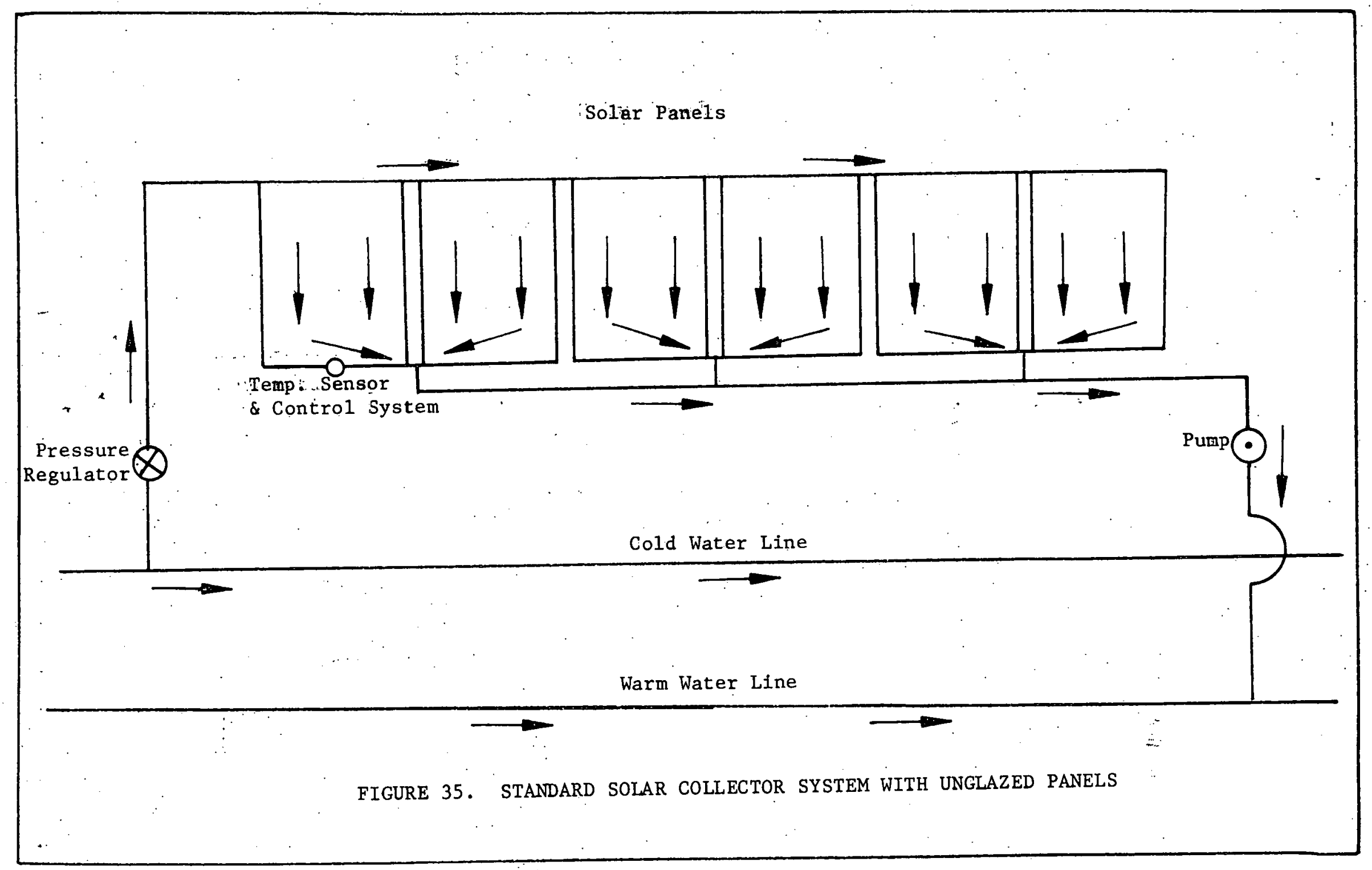




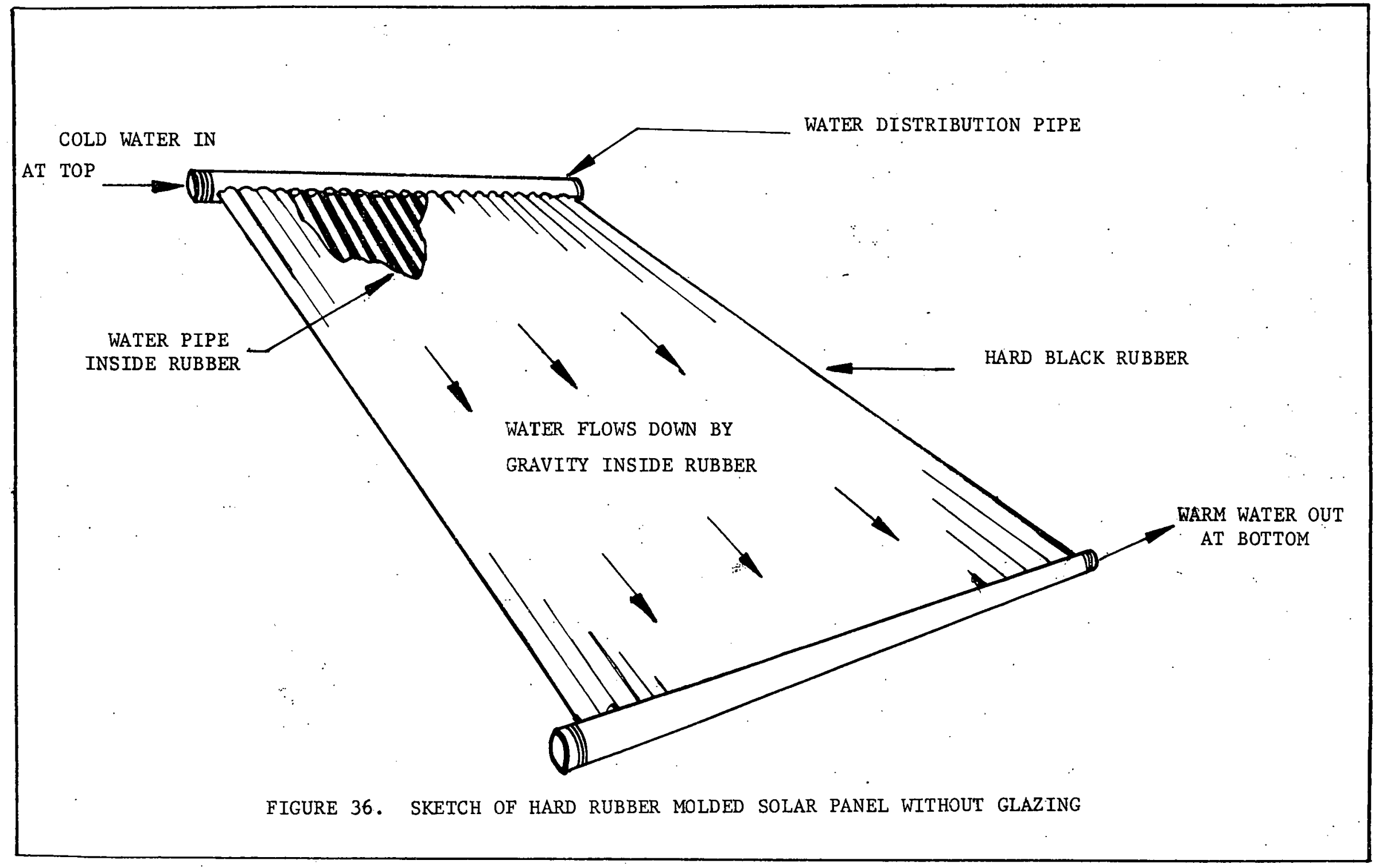




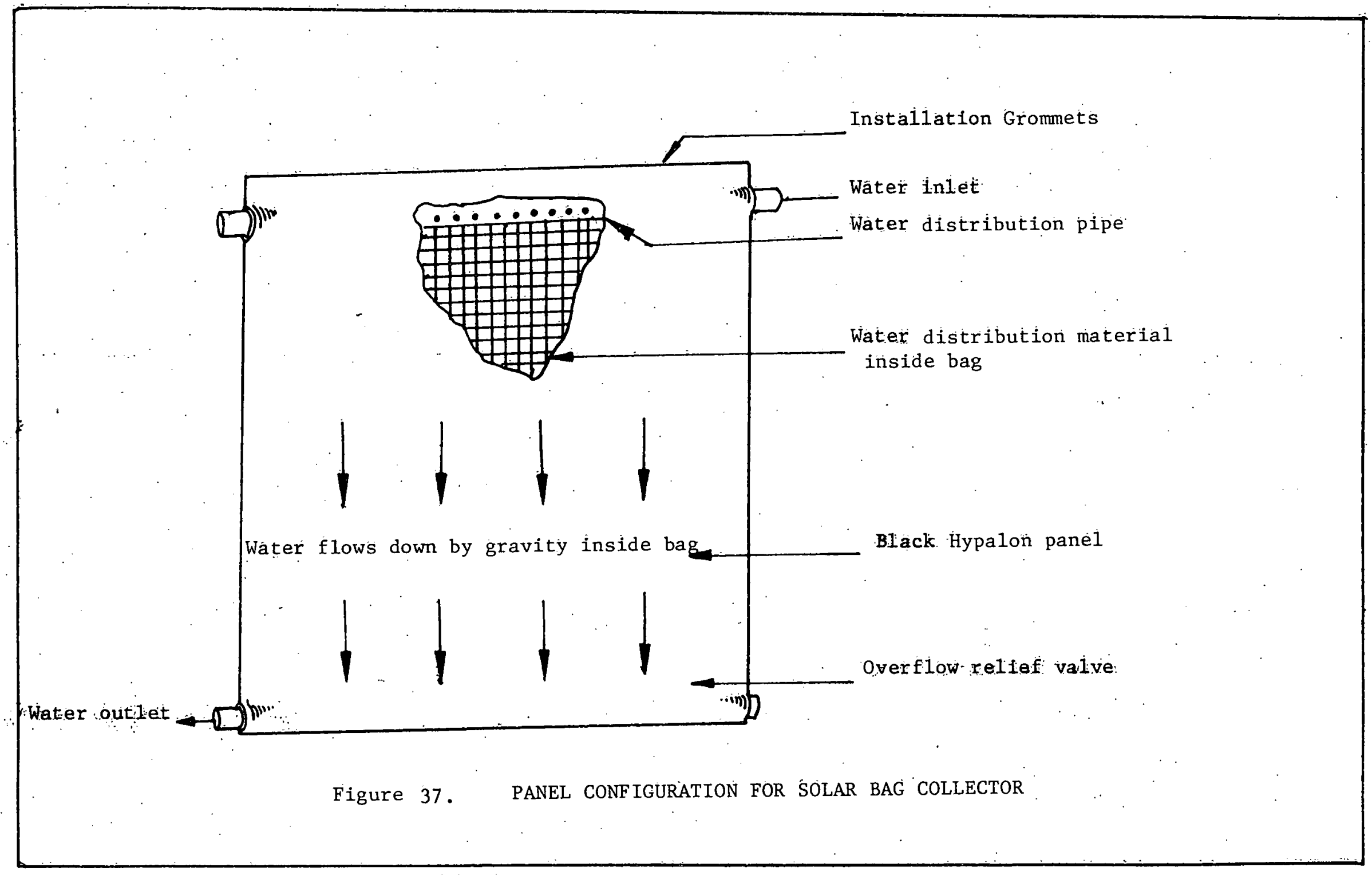




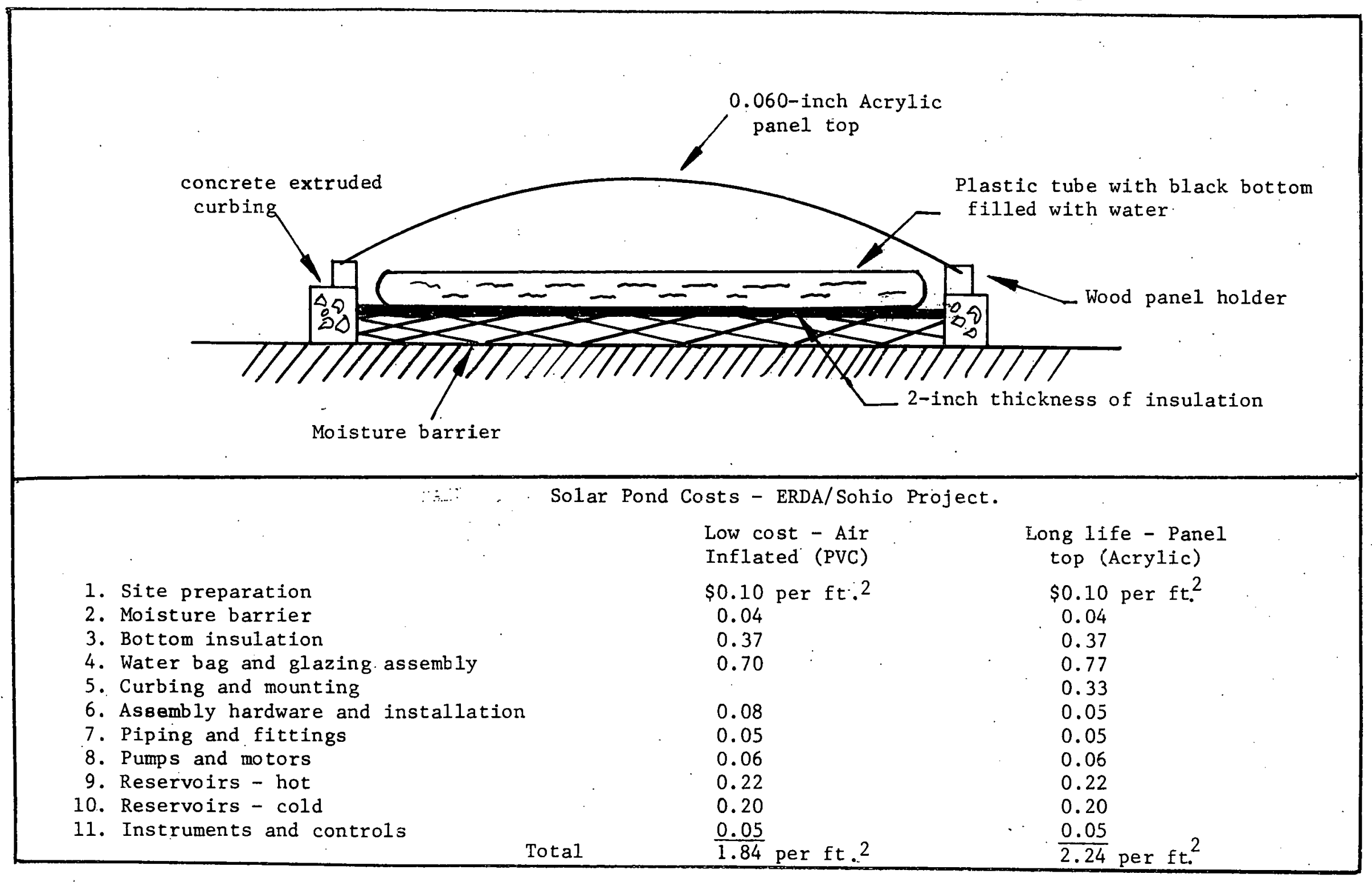


mally be 4-inch and 2-inch lines. The larger system costs double the minor system costs using cast iron pipes. Some PVC pipe is being used in $2,4,6$, and 8 -inch diameters. Pumping capacity could be cut by $40 \%$ if fire protection is not included.

The connected module integrated community energy system has the capacity to provide the required water for short periods (time meașured in days). Primary advantages are shorter pumping distances and relatively lower pumping requirements. Fire plugs utilizing both lines of the 2-pipe system could be installed. An additional check valve to each line would be required to eliminate cross flow. By reducing pressure in the lines with the fire engine pump, the submerged pumps will more than double the flow capacity. The 2 wells in a module will pro-. vide over 800 GPM under these conditions. Five connected modules will. provide water for most any emergency.

Small neighborhoods without city water have the potential for fire protection water with this system. Such systems would reduce fire insurance on the average home by over $\$ 100$ per year, more than paying the utility fee for heating and cooling water usage. The fire protection possibility shows at least one fringe benefit available from the system.

Long term water withdrawal such as for irrigation has not been considered because of the large requirement. Using the water as potable water is not considered inasmuch as treating and monitoring installations would be required for each module. However, treated potable water could be provided using a separate'but parallel supply system, eliminating the need for additional wells for drinking water. 
X. References

1. Naturà Atlas of the United States of America, U.S. Department of Interior, Geological Survey, 1970

2. Statistical Abstract of the United States, 1977 , Bureau of Census, U.S. Department of Commerce, 1977

3. Energy Perspectives, U.S. Department of Interior, 1975

4. Compressor Performance curves and tables by Tecumseh, transfered through American Air Filter, November 1978

5. Van Wyler, G.J. and Sonntag, R.E., Fundamentals of Classical Thermodynamics, John Wiley \& Sons, Inc., New York (1973)

6. Cropsey, M.G., "Analysis of Soil as Heat Source for Heat Pump System", Transactions of the ASAE, pp. 846-848, 1966, ASAE

7. Groethe, S.P., Sutton, G.E. and Liffler, W.A., "Earth as a Heat Source and Sink for Heat Pumps," Engineering Progress at the University of Florida; Vo1. IV, No. 6, June, 1950, Florida Engineering and Industrial Experiment Station

8. Krumme, V.W., "Luft-Wasser-Wärmepumpe in Einfamilienkaus", Elektrowärme International 32 (January 1974) Al.

9. Michels, V.F., "Probleme einer Luft-Wasser-Wärmepumpe bei tiefen Aussentemperaturen", Bull. SEV/VSE 68 (April 1977) 8,16

10. Christian, J.E., "Unitary Air-to-Air Heat Pumps", ANL/CES/TE/77-10, Oak Ridge National Laboratory, Oak Ridge, Tennessee (July, 1977).

11. Christian, J.E., "Unitary Water-to-Air Heat Pumps", ANL/CES/TE/77-9, Oak Ridge National Laboratory, Oak Ridge, Tennessee (October, 1977)

12. Skrotzke, B.G.A., Vapat, W.A., Power Station Engineering and Economy, McGraw-Hill, 1960

13. Huff, F.A., and Changnon, S.A., "Precipitation Modification by Major Urban Areas", Bulletin American Meteorological Society, Vol. 54, No. 12, December, 1973

14. Changnon, S.A., "Urban-Industrial Effects on Clouds and Preciptation", Proceeding on Inadvertant Weather Modification, Utah State University, 1973

15. Krauskopf, K.B., Introduction to Geochemistry, McGraw-Hill Book Company, (1967) 
16. Grubbs, D.M., Haynes, C.D., Hughes, T.H., and Stow S.H.,

"Compatibility of Subsurface Reservoirs with Injected Liquid

Wastes", NRC Report 721, The University of Alabama (June, 1972)

17. Todd, D.K., Groundwater Hydrology, John Wiley \& Sons, Inc., New York (1963)

18. Craft, B.C. and Hawkins, M.F., Applied Petroleum Reservoir

Engineering, Prentice - Hall, Inc., Englewood Cliffs, New Jersey (1959)

19. Gannon, R., "Ground-water Heat Pumps: Home Heating and Cooling From Your Own Well", Popular Science, pp. 78-82 (February 1978)

20. Henry, H.R., McDonald, J.R., and Alverson, R.M., "Aquifer Performance Tests Under Two-Phase Flow Conditions", BER Report 131-118, The University of Alabama (March, 1971)

21. Cormary, Y., et.al., "Heat Storage in a Phreatic Aquifer: Campuget Experiment", Electhecite! de France

22. Moly, F.J., Warman, J.C., and Thomas E.J., "Aquifer Storage of Heated Water: Experimental Study", Auburn University

23. Hunsaker, J.C. and Rightmire, B.G., Engineering Applications of Fluid Mechanics, McGraw-Hil1, New York (1947)

24. Rauscher, M., Introduction to Aeronautica1 Dynamics, John Wiley \& Sons, Inc., New York (1953)

25. Crocker, S., Piping Handbook, McGraw-Hill, New York (1945)

26. Means, R.S:, Building Construction Cost Data, Robert Snow Means Co., Inc. Duxbury, Massachusetts (1978)

27. Knudson, J.G., Personal Communication, Oregon State University, June 1978

28. Morse, R.W., and Knudson, J.G., "Effect of Alkalinity on the Scaling of Simulated Cooling Tower Water", The Canadian Journal of Chemical Engineering, Vo1. 55, June 1977

29. Knudson, J.G., and Story, M., "The Effect of Heat Transfer Surface Temperature on the Scaling Behavior of Simulated Cooling Tower Water", AICHE Symposium Series, No. 174, Vo1. 74

30. Lee, S..H. and Knudson, J.G., "Scaling Characteristics of Cooling Tower Water", Department of Chemical Engineering Oregon State, August 1978

31. Lamoreaux, P.E., Personnel Communication, October 1978, Data compiled from Alabama State Geological Survey Data 
32. Bennett, I., "Monthly Maps of Mean Daily Insolation for the United States", Solar Energy, Vol. 9, 1965

33. Liu, B.Y. and Jordan, R.C., "A Rational Procedure for Predicting The Long-Term Average Performance of Flat-Plate Solar-Energy Collectors", Solar Energy, Vo1. 7, No. 2 (1963)

34. "Burke Solar Heater", Burke Rubber Company, San Jose, California

35. "Energy From the Sun in Your Back Yard", Fayco, Inc., Menlo Park, California

36. Day, J.A., Clarke, A.F., Dickenson, W.C., and Iontuono, A., Industrial Process Heat from Solar Energy, IECE C 75 Record

37. Jepsson, R.W., Analysis of Flow in Pipe Networks, Ann Arbor Science, Ann Arbor (1976)

Additional References Used For Data And General Background Information

38. ASHRAE 1975 Handbook of Equipment, American Society of Heating Refrigeration, and Air Conditioning Engineers, New York (1975)

39. ASHRAE 1976 Handbook of Systems, American Society of Heating Refrigeration, and Air Conditioning Engineers, New York (1976)

40. ASHRAE 1977 Handbook of Fundamentals, American Society of Heating Refrigeration, and Air Conditioning Engineers, New York (1977)

41. ASHRAE 1978 Handbook of Applications, American Society of Heating Refrigeration, and Air Conditioning Engineers, New York (1978)

42. Strock, C., Handbook of Air Conditioning, Heating and Ventilating, The Industrial Press, New York (1959)

43. Christian, J.E., "Central Cooling - Compressive Chillers", ANL/ CES/TE 78-2, Oak Ridge National Laboratory, Oak Ridge, Tennessee (March, 1978)

44. Baumeister, T., and Marks, L.S., Standard Handbook for Mechanical Engineers, McGraw-Hil1, New York (1967)

45. Fink, D.G. and Carroll, J.M., Standard Handbook for Electrical Engineers, McGraw-Hil1, New York (1968)

46. Meyer, C.F., "Status Report on Heat Storage Wells", Water Resources Bulletin (April 1976), American Water Resources Association

47. Kormi, I and Keenan, J.D., "Energy Recovery in Browery Industry", Journal of The Environmental Engineering Division, pp. 445-459

(June 1977) 
48. Labor, II., "Solar Ponds: Large-Area Solar Collectors for Power Production", Solar Energy, Vol. 7, No. 4, pp. 189-194 (1963)

49. Weinberger, H., "The Physics of the Solar Pond", Solar Energy, Vo1. 8, No. 2, pp. 45-56́ (1964)

50. Dickinson, W.C., Clark, A.F., Day, J.A. and Wouters, L.F., "The Shallow Solar Pond Energy Conversion System", Solar Energy, Vo1. 18, pp. 3-10 (1976)

51. Comly, J.B., Jaster, H, and Quaile, J.P., "Heat Pumps-Limitations and Potential", GE Report 75 CRD185, General Electric Company, Schenectady, New York (September 1975)

52. Kennedy, A.S., and Tschanz, J.F., "Factors that Influence the Acceptance of Integrated Community Systems", Argonne National Laboratory, Argonne, Illinois (January 1978)

53. "Heat Pump System Combine's Features of Unitary and Central Approaches", Architectural Record, October (1969)

54. Olivieri, J.B., "Internal Source Heat Pumps and Heat Recovery", ASHRAE Journa1 (October 1967)

55. Cavalleri, G. and Galigno, G., "Proposal for the Production and Seasonal Storage of Hot Water to Heat a City", Solar Energy, Vo1. 19

56. Whitehead, E.R., "Using Heat Pumps for Energy Recovery" Plant Engineering (April 14, 1977)

57. Hise, E.C. and Wilson, J.V., "A Heat Pump Cycle with an AirWater Working Fluid", 12th IECEC, Vol. 1, (1977)

58. "Heat Pump Technology: A Survey of Technical Developments, Market Prospects and Research Needs", HCP/M2121-01, U.S. Government Printing Office, Washington, D.C. (June 1978).

59. Becker, H.P., "Energy Conservation Analysis of Pumping Systems", ASHRAE Journal (April 1975)

60. "Feasibility of a District Cooling System Using Natural Cold Water", ANL/ICES-TM-10, Tracor JITCO (December 1977) 
XI. APPENDIX I. ANNUAL HEATING AND COOLING PERFORMANCE COMPUTER PROGRAM SUMMARY

This program is designed to compute annual heating and cooling efficiencies and energy requirements for any specific combination of heating/coollng unit, building, and city. It accomplishes this using input data describing heating/cooling system performance, building design parameters, and detailed weather data for specific cities. It can represent a home or major building by changing internal energy input.

Weather data used in the program have been abstracted from published NOAA reports (hours in $5^{\circ} \mathrm{F}$ temperature increments) and ASHRAE tables (99\% design temperatures) to give, for each city, 30 parameters. These consist of 28 values for the number of hours per year in which temperature falls within 5 degree increments, starting at $-18^{\circ} \mathrm{F}$; the last two items are cooling and heating design outdoor temperatures, respectively. Heating and cooling design temperatures are $99 \%$ values taken from ASHRAE Fundamentals Handbook.

System parameters are abstracted from manufacturer's data for production systems, interpolated as necessary to fill the same $5^{\circ} \mathrm{F}$ increments used for weather data. Information stored for each $5^{\circ} \mathrm{F}$ temperature increment includes:

$$
\begin{aligned}
& \text { 1. COOLING CAPACITY - MBTUH } \\
& \text { 2. COOLING POWER INPUT }-\mathrm{KW} \\
& \text { 3. HEATING CAPACITY }-\mathrm{MBTUH} \\
& \text { 4. HEATING POWER INPUT }-\mathrm{KW}
\end{aligned}
$$

For maximum flexibility 5 inputs are required to specify building design conditions. These are: 
1. Design Cooling Load - heat gain due to convection, radiation, and conduction at given design conditions

2. Design Cooling Indoor Temperature - the indoor temperature to be maintained during cooling plant operation

3. Design Heating Load - heat loss from building due to conduction, convection, and radiation under given design conditions

4. Design Heating Indoor Temperature - indoor temperature to be maintained during heating mode

5. Internal Load - heat gain in building due to occupants, machinery, lights, etc.

It was felt that these represent the minimum information necessary for a satisfactory working description of building loads.

The program's basic calculations assume that during both heating and cooling, heat transfer between a building and its environment is directly proportional to the temperature difference between indoors and the environment, so that:

Heat Load Due to Heat Transfer=(Design Load) (Thermostat temp.-Outdoor temp.) (Design indoor temp.-Design outdoor temp.) and (Thermostat temp.-Design indoor temp.) Cooling Load Due to Heat Transfer=(Design Load) (Outdoor design temp.-Design indoor temp.)

Then:

Total Heating Load $=$ (Heat Load Due to Heat Transfer) - Internal Load Total Cooling Load $=$ (Cooling Load Due to Heat Transfer) + Internal Load The program combines this information along with system performance data to calculate system run times as follows. First it computes heating load. If negative, it computes cooling load. If that too is negative then the program assumes the heating/cooling unit will be off. Otherwise it divides the load by system capacity to get run time in percent. If run time computed is over $100 \%$ for heating mode, resistance heat is added to reduce it to $100 \%$. If $100 \%$ run time is exceeded in the cooling calcula- 
tion, run time is reduced to $100 \%$ and cooling load is restricted to system capacity. Run time is multiplied by system power requirements and the number of hours in each $5^{\circ} \mathrm{F}$ increment to give seasonal power input at that temperature. Seasonal temperature hours at temperature are. also multiplied by heating/cooling loads to give seasonal loads. Seasonal values are summed for computation of annual values of heating load, cooling load, annual heating input, and annual cooling input. Coefficients of performance are also calculated,

$$
\text { COP }=\frac{\text { Total Load }}{\text { Total Power Input }}
$$

as are equivalent heating fuel values, which assume $62 \%$ furnace efficiency for fossil fuel furnaces.

$$
\text { Equivalent fuel required }=\frac{\text { Total Heating Load }}{\text { Heat Content per unit fuel } \times 62 \%}
$$

The program also incorporates provisions for loops to accomplish serial solutions for varying cities, systems, and design conditions. A table defining the variables in the computer programs, a flow chart, and a program listing follow. 
PROGRAM VARIABLES

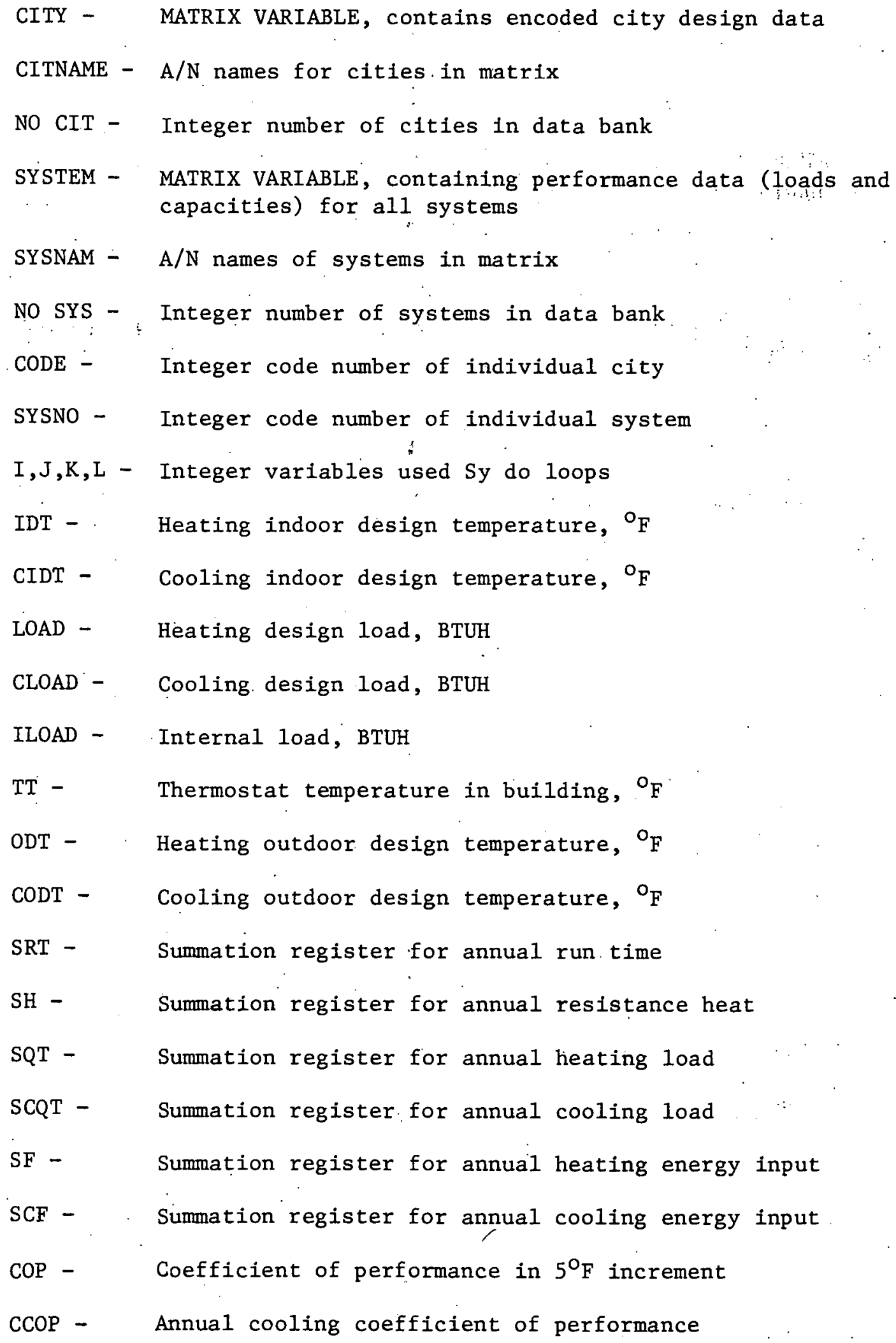


$\mathrm{COPH}-\quad$ Annual heating coefficient of performance

EGAS - Equivalent annual gas heat required

EOIL - Equivaient annual oil heat required

A - $\quad$ Outdoor temperature, ${ }^{\circ} \mathrm{F}$

B - System heating/cooling capacity, MBTUH, at outdoor temperature A

C - Heat pump compressor run time in $5^{\circ}$ increment, percent

D - Input for heat pump at temperature $\mathrm{A}, \mathrm{KW}$

$\mathrm{E}$ - Seasonal heating hours in $5^{\circ} \mathrm{F}$ increment about A $\left(\mathrm{A} \pm 2.5^{\circ} \mathrm{F}\right)$

F - Seasonal heat pump input in $5^{\circ}$ increment about $A$

G - Resistance heat required in $5^{\circ} \mathrm{F}$ increment about $\mathrm{A}$

$\mathrm{H}$ - Seasonal resistance heat needed in $5^{\circ} \mathrm{F}$ increment about $\mathrm{A}$

QT - Seasonal heating/cooling load in $5^{\circ} \mathrm{F}$ increment about $\mathrm{A}$ 


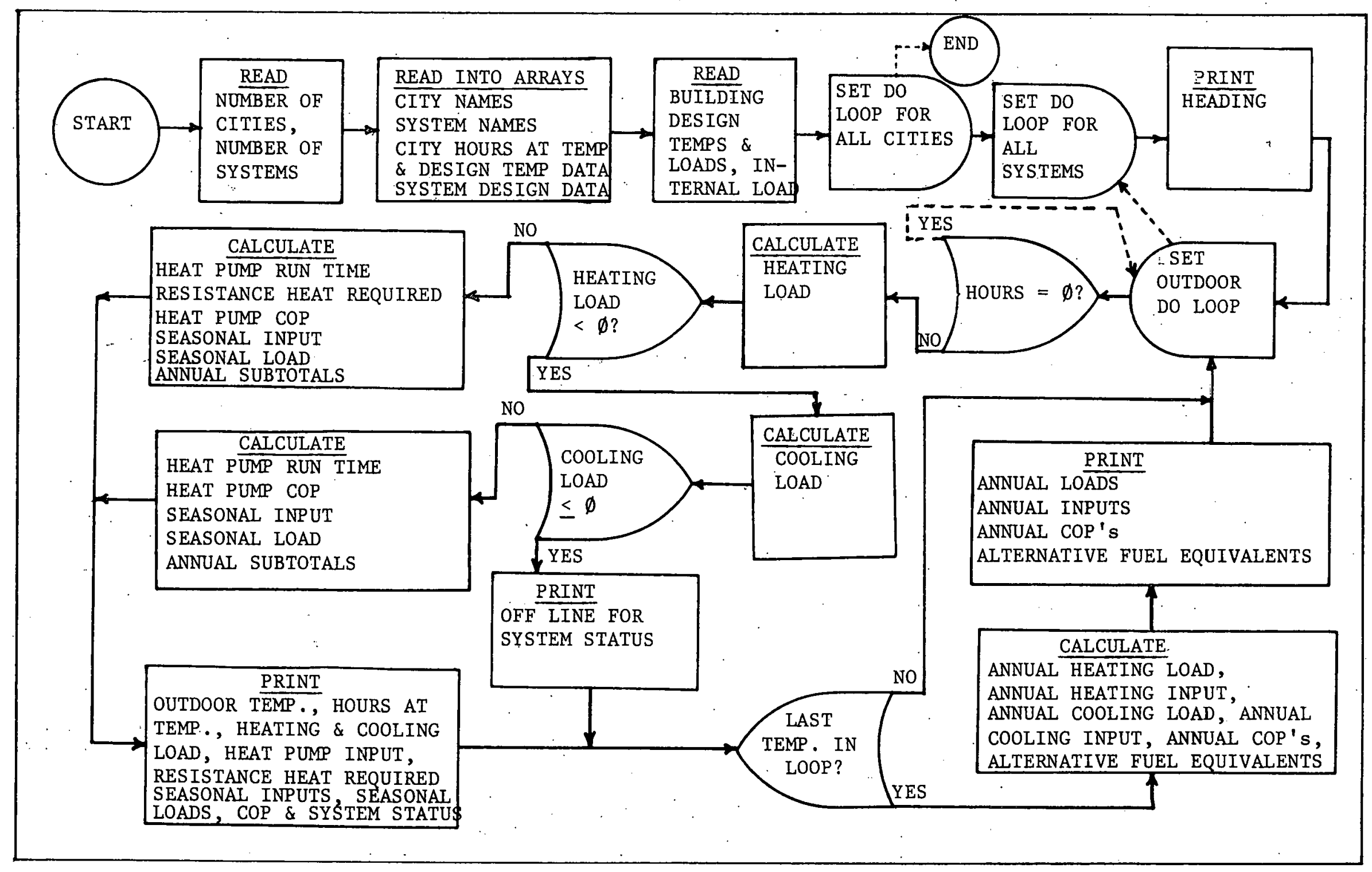


C REATING 8.COULING SYSTEM PERFORMANCE

DIMENSION CITY $(28,30), C \operatorname{ITNAM}(28,5), \operatorname{SYSTEM}(5,4,30), \operatorname{SYSNAM}(5,5)$

INTEGER COOE, SYSINO

REAL LOAD, A, B,C,D,E,F, G,H,Q, QT, IDT, ILOAD

READ (5,100) NOCIT, NOSYS

DO 5 CODE $=1$, NOCIT

READ $(5,119)$ (CITNAM $(C O D E, K), K=1,5)$

5 CONTINUE

DO O I $=1$, NOSYS

$\operatorname{READ}(5,119)(\operatorname{SYSNAN}(I, J), J=1,5)$

- CONTINUE

DO $10 I=1$, NOCIT

$\operatorname{READ}(5,100)(\operatorname{CITY}(1, J), J=1,30)$

10 CONTINUE

DO $20 \quad I=1$, NOSYS

DO $90 \mathrm{~J}=1.4$

READ $(5,100)$ (SYSTEM $(I, J, K), K=1,28)$

90 COINTINUE

¿U CONTINUE

REAU $(5,100)$ IOT , CIUT, LOAD, CLOAD, TT, ILOAD

WRITE $(6,200)$

200 FORMAT 1 '0'20X,'UNIVERSITY OF ALA. $1 \% 11,15 X$, 'HEATING ANU AIRCO

\&NDITIONING PERFORMANCE ANALYSIS' $/, 1,9 X, '$ SCHAFTZLE \& LECROY')

DO 210 COUE $=1$, NOCIT

DO 220. SYSNO $=1$, NOSYS

WRITE $(6,3 \cup 0), C O D E,(C I T N A M(C O D E, L), L=1,5),(\operatorname{SYSNAM}(S Y S N O, M), M=1,5)$

300 FOKMAT $(1,15 X$, 'THIS PROGRAM WILL ANALYZE PERFORMANCE AT LOCATION',

\&I3.',',5A4, 'FOR ', JA4, 'HEATING/COOLING SYSTEM')

WRITE $(6,301)$ TT,ILOAD

$30 \perp$ FORMAT' ' 'CALCULATIONS ARE FOR INDOOR TEMPERATURE MAINTAINED AT

\&'F4.0,'F ANO INTERNAL LOAD OF ',F6.0.' BTUH')

100 FORIMAT (1)

COUT $=$ CITY $(C O D E, 30)$

ODT $=$ CITY $(C O D E \cdot 29)$

WRITE $(6,700)$ ODT,LOAD, IDT

700 FORMAT' 10 ' HEATING CALCULATIONS ARE FOR OUTDOOR DESIGN TEMP. OF', $\& F 4.0 .1 F$ ANO LOAD OF ', F8.0,' BTUH AT DESIGN INDOOR TEMP. OF',F4. $\& 0,1 F$,

WRITE $(6,1100)$ COUT,CLOAD,CIDT

1100 FORMAT 1,1 'COOLING CALCULATIONS ARE FOR OUTDOOR DESIGN TEMP. OF' \&,F4.0,' F AND LOAO OF',F8.0, 'BTUH AT. DESIGN INDOOR TEMP. UF, F4.0, \&' $F, 1)$

WRITE $(6,8 \cup \cup)$

BUO FORMAT 1 'U','A= UUTDUOR TEMPERATURE,FAHREINHEIT', $, \cdot 1, \cdot B=$ SEASONAL \& HEATING HOURS IN 3 DEGREE INCREMENT $, 1 \% \cdots, 1 \%$ SEASONAL HEATING \&COULING LUAD IN J UEGREE INCREMENT , BTU', 
$\&$

$1,1, \cdot D=$ HEAT PUMP CAPACITY

\&AT OUTDOOR TEMPERATURE A, MBTUH',

$\&$

$1,1,1 E=$ HEAT PUMP SYSTEM INPUT, KW, IN \&CLUDING AIR HANDLER REQUIREMENT, $K W 1$,

$\&$. QNPERCENT AT TEMPERATURE A' Q $, \because \because, F=$ HEAT PUMP CONPHESSOR RUN TIME I $\because, \cdots \cdot \because=$ SEASONAL HEAT PUMP INPUT, KWH IN 5 DEGREE TEM ¿PERATURE INCKEMENT', $/, 1$, ¿STANCE HEAT REQULKLD IN 5 DEGREE INCREMENT, $K W \cdots, \cdots, I=$ SEASONAL \& RESISTANCE HEAT NEEDED IN 5 DEGREE INCREMENT $1,1,1 J=$ COEFICIEN \&T OF PERF ORMAINCE, UIMENSIONLESS $1,1, ? ' K=$ SYSTEM STATUS', $1,101,40$ $\& X$, STANDARO CALCULATION $, 1,: 01,9 X$, "A $\&$

$S R T=0$.

E $F$ G

$H$

$B$

$S H=0$.

SOG $=0$.

$S F=0$.

SQT $=0$.

$S C F=0$.

SCUT $=0$.

$A=-18$.

DO $50 \quad M=1.28$

$115 E=C I T Y(C O D E, M)$

$\operatorname{IF}(E \cdot L E \cdot 0$.$) GO TO 49$

LEEST $=0$

IF $(A-T T) \quad 111 \cdot 112 \cdot 113$

$111 B=S Y S T E M(S Y S I N O, 3, M)$

$D=S Y S T E M(S Y S N O, 4, M)$

LEST $=L E S T+1$

IF(LEST.GT.2) GO TU 112

$Q=(L O A D /(I D T-O O T)) *(T T \quad-A)-I L O A D$

IF (Q.LT.0.) GO TO 113

IF $(Q . E Q .0$.$) GO TO 112$

$C=(Q / B) / 10$.

IF $(C \cdot G E \cdot 100) \quad C=$.100 .

$Q T=E * Q$

$G=(Q-(B * 10 \cdot * C)) / 3412$.

$\operatorname{IF}((Q-(B * 1000).) \cdot L E \cdot 0) \quad G=$.0 .

$H=E * G$

$F=E * D * C / 100$.

$S F=S F+F$

$S Q T=S Q T+Q T$

$\mathrm{SH}=\mathrm{SH}+\mathrm{H}$

$C O P=(B+3.412 * G) /((U+G) * 3.412)$

WRITE $(6,900) A, E, Q T, B, D, C, F, G, H, C O P$

900 FORMAT (! ', 2X.2F9.U.F10.0 ,F9.1,F9.2,F9.1,FG.1,F9.1,F9.1,F9.2, 3X \&. 'HEATING')

GO TO 49 


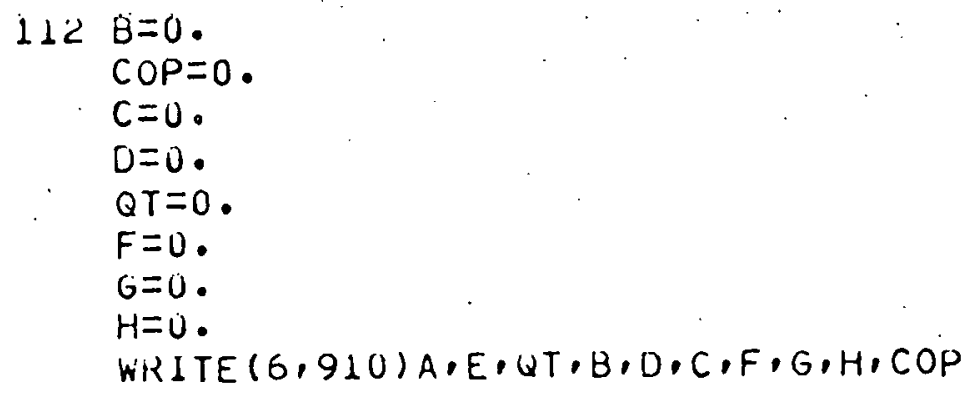

910 FORMAT(, ',2X.2F9.U.F10.0 ,F9.1.F9.2,F9.1,FG.U.F9.1.F9.1,F9.2.3X \&. 'UFF')

GO TO 49

$113 B=5 Y S T E M(S Y S N O, 1, M)$

LEST $=L E S T+1$

$D=S Y S T E M(S Y S N O, 2, M)$

IF (LEST.GT.2) GO TO 112

$Q=(C L O A D /(C O D T-C I D T)) *(A-T T)+I L O A D$

$\operatorname{IF}(Q \cdot L T \cdot 0$.$) GO TO 111$

IF $(Q \cdot E Q \cdot 0 \cdot)$ GO TO 112

$C=(Q / B) * \cdot 1$

IF $(C \cdot G E \cdot 100) \quad C=,1 \cup 0$.

IF $(Q \cdot G E \cdot(B * 1000 \cdot)) \quad Q=B * 1000$

$C Q T=E * Q$

$C F=E * D * C * .02$

$G=\dot{U}$.

$H=U$.

$S C F=S C F+C F$

$S C U T=S C Q T+C Q T$

CUP $=B /(D * 3.412)$

WRITE $(6,920)$ A,E,CET, B,D,C,CF, G, H, COP

920 FOKMAT(' ',2X,2F9.UंF10.0 ,F9.1,F9.2,F9.1,F9.0.F9.1,F9.1,F9.2.3X \&. 'COOLING')

$49 A=A+5$.

50 CUNTINUE

COPH $=(S Q T /(S H+S F)) / 3412$.

$C C U P=S C Q T /(S C F * 3412 \cdot)$

EGAS=SQT $\quad /(62000$.

EOIL=SQT /(145000.*.62)

WRITE $(6,1000)$ SQT,SF, SH, SCQT,SCF,CCOP,COPH

.000 FORMAT $(10 \%$, ANNUAL HEATING LOAD, BTU $=1, F 10.0 \%, 1,1$ ANNUAL HEATI \&NG HEAT PUMP INPUT, KWH $=1, F 10.0,1, \cdots$ 'ANNUAL RESISTANCE HEAT RE \&QUIRED. KWH $=1, F 10.0 .1 .1$, ANNUAL COOLING LOAח BTU $=1 ., F 10.0 .1$, \&' 'ANNUAL COOLING HEAT PUMP INPUT, KWH $=1, F 10.0 \% 1,1$, ANNUAL C \&OOLING COP $=1, F G . \angle 1 /, \cdots$ ANINUAL HEATING COP $=(, F G .2)$ WRITE $(6,211)$ EGAS,EOIL

211 FORMAT(' ', 'GAS HEAT AT 62X EFFICIENCY WOULD REQUIRE',F10.0,' THER \&MS.' ', ', ',FUEL UIL HEAT AT 62\% EFFICIENCY WOULD REQUIRE ',F6.0, \& ' UALLONS')

119 FOKMAT (5A4)

220 CONTINUE

210 CONTINUE 
159

XII. APPENDIX II. COMPUTER PROGRAM FOR DETERMINING PRESSURE LOSS IN PIPING SYSTEM DUE TO FRICTION

A computer program has been developed to analyze pipe friction losses in the piping system. The program uses the Darcy-Welsback equation as a basis. The variables utilized in the program are pipe diameters, pipe lengths, flow rates, and extraction flow rates. The pipe roughness for PVC pipe and viscosity for water are included as constants.

The co-owned program by Jepperson performs a friction factor analysis by approximating the Moody diagram for pipe flow. ${ }^{37}$ The program determines if the flow is turbulent or laminar, determines the friction factor, and then calculates pressure losses. A flow chart, program variables, and listing of the program follow. 


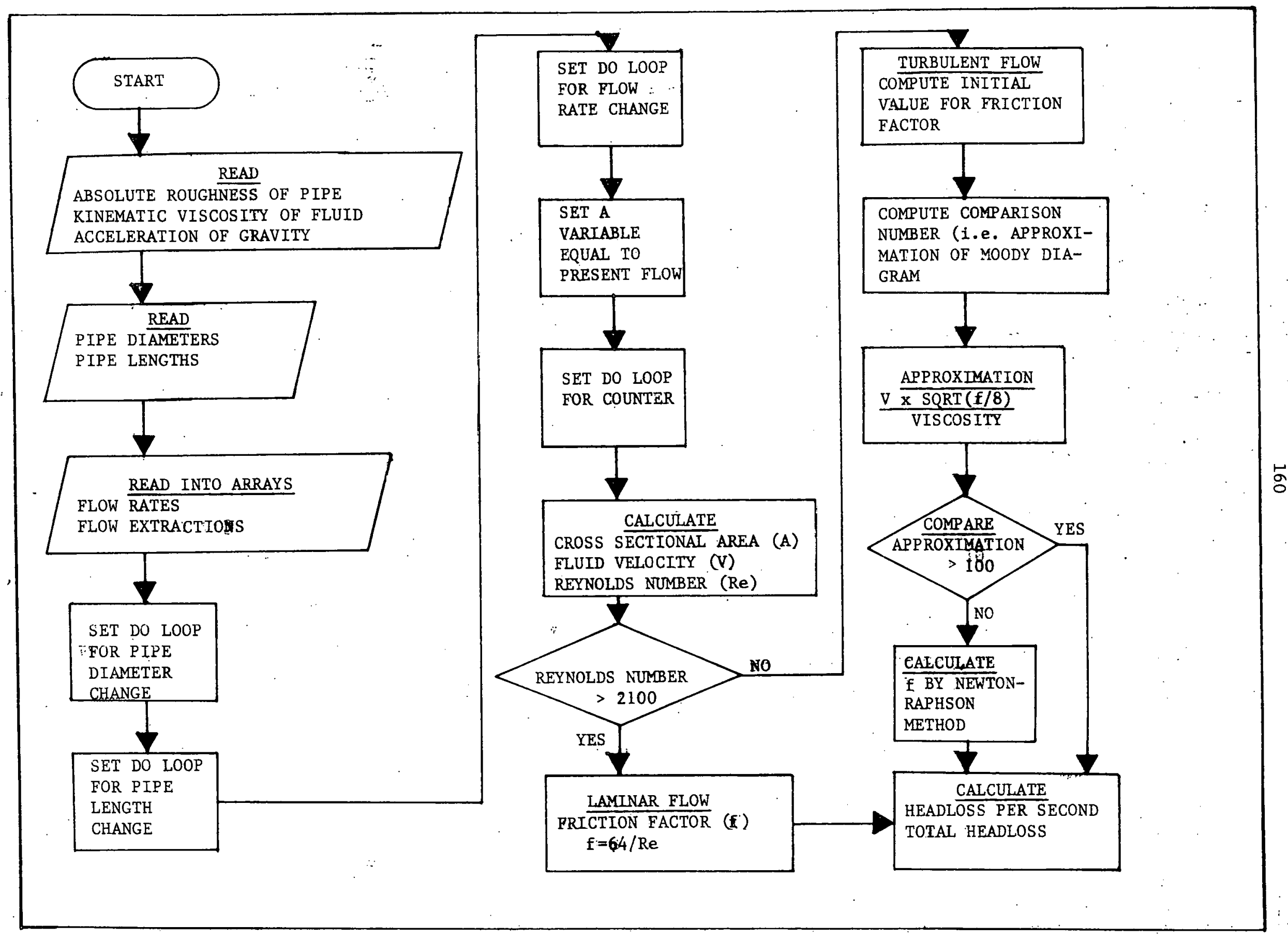




\section{PROGRAM VARIABLES}

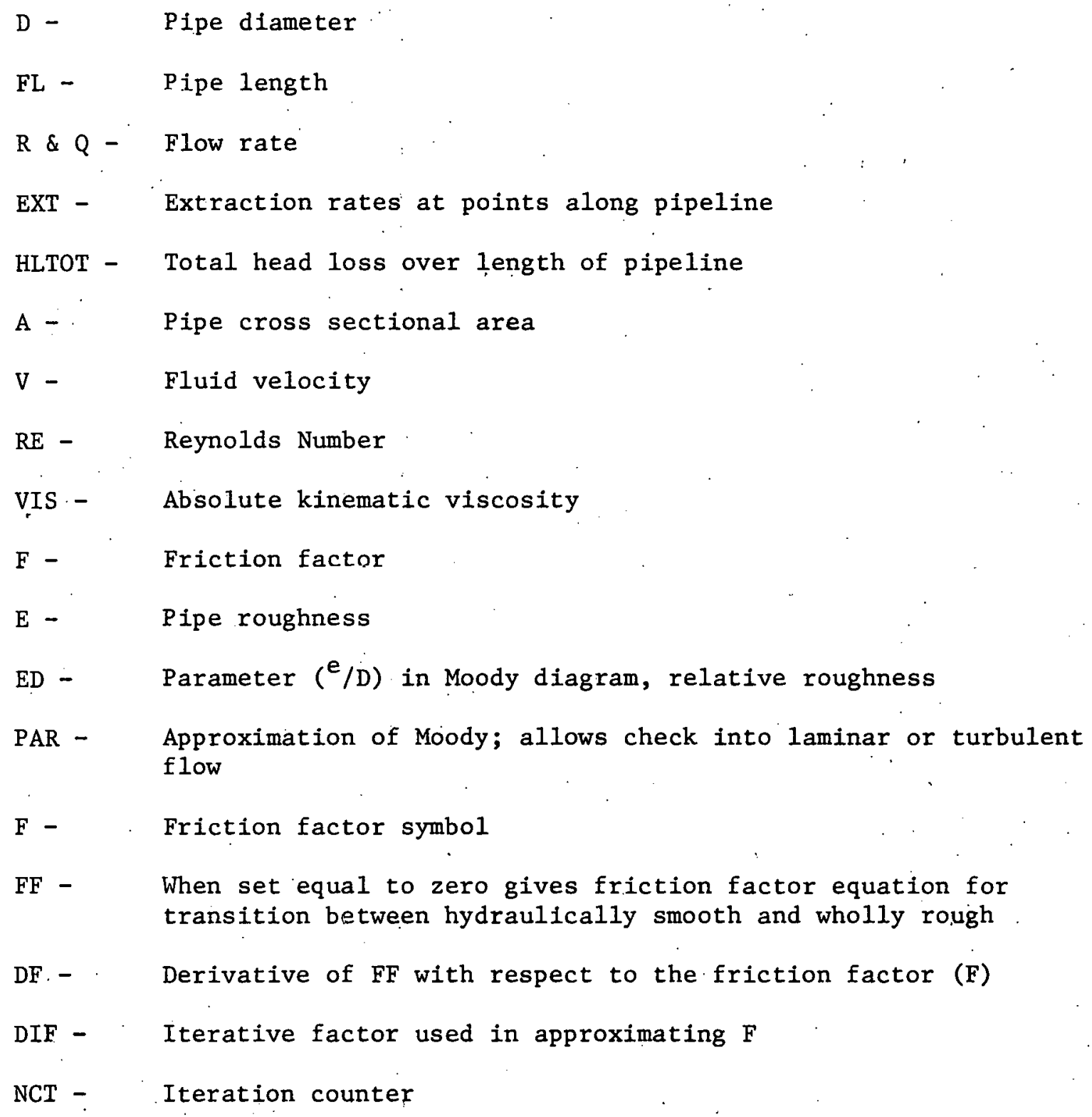


DIMENSION D ( 5),FL (2),R(3),EXT(3)

READ (5,*)VIS,E, G

$\operatorname{READ}(5, *)(D(I), I=1,5),(F L(J), J=1,2)$ :

$\operatorname{REAO}(5, *)(R(K), K=1,3),(\operatorname{EXT}(L), L=1,3)$

DO $20 \quad I=1,5$

DO $20 \mathrm{~J}=1,2$

DO $20 \mathrm{~K}=1,2$

$Q=R(K)$

HILTOT $=0$.

DO $20 \mathrm{~L}=1,10$

C D-PIPE OIAMETER, Q-FLOW RATE,FL-LENGTH OF PIPE,EXT-EXTRACTION RATE

$C$ VIS-KINEMATIC VISCOSITY OF FLUID $X 10 * 25$,

C E-ABSOLUTE ROUGHINESS OF. PIPE, G-ACCELERATION OF GRAVITY

$A=.7839816 * D(I) *[$ (I)

$V=Q / A$

$R E=V * D(I) / V I S$

IF $(R E . G T .2100)$ GO TO 3

$F=64 . / R E$

$3 \quad$ EVIS $=E / V I S$

$E L O G=9.35 * A L O G 10(2.71828183)$

$E D=E / D(I)$

$F=1 . /(1.14-2 \cdot * A L O G 10(E D)) * * 2$

$P A R=V * S O R T\left(F / 3_{0}\right) * E V I S$

IF (PAR.GT.10U.) GO TO 1

INCT $=0$

$2 \quad F S=S Q R T(F)$

$F Z=.5 /(F * F S)$

$A R G=E D+9.35 /(R E * F S)$

$F F=1 . / F S-1 \cdot 14+2 . * A L O G 10(A R G)$

$L F=F Z+E L O G * F Z /(A K G * R E)$

$D I F=F F / D F$

IF (ABS (DIF).GT..00001.AND.NCT.LT.15). GO TO 2

$\mathrm{F}=\mathrm{F}+\mathrm{DF}$

$\therefore \quad: H C T=N C T+1$

$1 \quad H L=F * F L(J) * V * V /(\tilde{c}, * G * D(I))$ WRITE $(6,101) Q, 0, F L(J), F, H L$

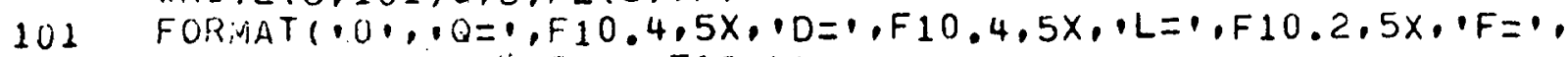
\&F10.5.5X, HEADLOSS $=,, F 10,4)$

HLTOT $=H L$ TOT $+H L$

IF (L.LT.10) 50 TO 20

WRITE $(6,102)$ HLTOT

102 FORMAT $(10,10 \times, E 10.6)$

$Q=Q-E X T(K)$

20 CONIINUE

99 STOP

EiNO 
Distribution

AIIL

P. T. Bauer

J. M. Calm (50)

A. S. Kennedy

T. J. Marciniak

J. Martusek :

M. Pac1 (12)

J. Pascual

V. A. Rabl

J. Tschanz

ANL Libraries (C. Archer)

TIS Files (E. N. Pettitt)

DOE

G. Anderson

S. Cavros

J. Kaminsky

J. C. Rodousakis (25)

I. Sewell

DOE-TIC (65)

\section{External}

C. E. Brett, University of Alabama (25)

V. R. Anderson, Billings Energy Corporation (3)

P. F. Swenson, Consolidated Natural Gas Service Company (3)

A. Halfon, Dubin-Bloome Associates (3)

H. G. Lorsch, Franklin Research Center (3)

D. W. Wade, Georgia Tech Research Institute (3)

P. A. Anderson, Honeywell Energy Resources Center (3)

L. L. Northrup, Northrup Incorporated (3)

L. B. Katter, Rocket Research Company (3) 